\title{
Structure-Activity Relationships of a Diverse Class of Halogenated Phenazines that Targets Persistent, Antibiotic-Tolerant Bacterial Biofilms and Mycobacterium tuberculosis
}

Aaron T. Garrison ${ }^{\dagger}$, Yasmeen Abouelhassan ${ }^{\dagger}$, Verrill M. Norwood IV ${ }^{\dagger}$, Dimitris Kallifidas ${ }^{\dagger}$, Fang Bai ${ }^{\ddagger}$, Minh Thu Nguyen ${ }^{\dagger}$, Melanie A. Rolfe ${ }^{\dagger}$, Gena M. Burch ${ }^{\dagger}$, Shouguang Jin $^{\ddagger}$, Hendrik Luesch ${ }^{\dagger}$, Robert W. Huigens III ${ }^{\dagger}$

${ }^{\dagger}$ Department of Medicinal Chemistry, College of Pharmacy, University of Florida, Gainesville, Florida 32610; ${ }^{\ddagger}$ Department of Molecular Genetics \& Microbiology, College of Medicine, University of Florida; ${ }^{\perp}$ Center for Natural Products, Drug Discovery and Development (CNPD3), University of Florida

\section{Supporting Information}

1.) General Information $\quad$ S2

2.) Synthetic Procedures and Characterization Data $S 3$

$\begin{array}{lr}\text { 3.) Biological Methods } & \text { S17 }\end{array}$

$\begin{array}{lr}\text { 4.) Literature References } & \text { S24 }\end{array}$

5.) Supporting Images of Biological Experiments $\quad$ S25

6.) ${ }^{1} \mathrm{H}$ NMR \& ${ }^{13} \mathrm{C}$ NMR Spectra for New Compounds $\quad$ S42 


\section{1.) General Information:}

All synthetic reactions were carried out under an inert atmosphere of argon unless otherwise specified. All reagents for chemical synthesis were purchased from commercial sources and used without further purification. Reagents were purchased at $\geq 95 \%$ purity and commercially available controls were used in our biological investigations without further purification. All microwave reactions were carried out in sealed tubes in an Anton Paar Monowave 300 Microwave Synthesis Reactor. A constant power was applied to ensure reproducibility. Temperature control was automated via IR sensor and all indicated temperatures correspond to the maximal temperature reached during each experiment. Analytical thin layer chromatography (TLC) was performed using $250 \mu \mathrm{m}$ Silica Gel $60 \mathrm{~F} 254$ pre-coated plates (EMD Chemicals Inc.). Flash column chromatography was performed using 230-400 Mesh 60^̊ Silica Gel from Sorbent Technologies. All melting points were obtained, uncorrected, using a Mel-Temp capillary melting point apparatus from Laboratory Services, Inc.

NMR experiments were recorded using broadband probes on a Varian Mercury-Plus-400 spectrometer via VNMR-J software (400 MHz for ${ }^{1} \mathrm{H}$ and $100 \mathrm{MHz}$ for ${ }^{13} \mathrm{C}$ ). All spectra are presented using MestReNova 8.1 (Mnova) software and are displayed without the use of the signal suppression function. Spectra were obtained in the following solvents (reference peaks also included for ${ }^{1} \mathrm{H}$ and ${ }^{13} \mathrm{C}$ NMRs): $\mathrm{CDCl}_{3}\left({ }^{1} \mathrm{H}\right.$ NMR: $7.26 \mathrm{ppm}$; ${ }^{13} \mathrm{C}$ NMR: $\left.77.23 \mathrm{ppm}\right), d_{6}$-DMSO $\left({ }^{1} \mathrm{H}\right.$ NMR: $2.50 \mathrm{ppm} ;{ }^{13} \mathrm{C}$ NMR: $\left.39.52 \mathrm{ppm}\right)$ and $d_{4}$-MeOD $\left({ }^{1} \mathrm{H}\right.$ NMR: 3.31 ppm; ${ }^{13} \mathrm{C}$ NMR: $49.00 \mathrm{ppm}$ ). All NMR experiments were performed at room temperature. Chemical shift values $\left(\delta\right.$ ) are reported in parts per million (ppm) for all ${ }^{1} \mathrm{H}$ NMR and ${ }^{13} \mathrm{C}$ NMR spectra. ${ }^{1} \mathrm{H}$ NMR multiplicities are reported as: $\mathrm{s}=$ singlet, br. $\mathrm{s}=$ broad singlet, $\mathrm{d}=$ doublet, $\mathrm{t}=$ triplet, $\mathrm{q}=$ quartet, $\mathrm{m}=$ multiplet. HighResolution Mass Spectrometry (HRMS) were obtained for all new compounds from the Chemistry Department at the University of Florida. The purities of all compounds evaluated in biological assays were confirmed to be $\geq 95 \%$ by LC-MS using a Shimadzu Prominence HPLC system, AB Sciex 3200 QTRAP spectrometer, and a Kinetex C18 column $(50 \mathrm{~mm} \times 2.1 \mathrm{~mm} \times 2.6 \mu \mathrm{m})$ with a 13-minute linear gradient from 10-80\% acetonitrile in $1 \%$ formic acid at a flow rate of $0.25 \mathrm{~mL} / \mathrm{min}$.

Bacterial strains used during these investigations include: methicillin-resistant Staphylococcus aureus (Clinical Isolate from Shands Hospital in Gainesville, FL: MRSA-2; ATCC strains: BAA-1707, BAA-44) methicillinresistant Staphylococcus epidermidis (MRSE strain ATCC 35984; methicillin-sensitive strain ATCC 12228), vancomycin-resistant Enterococcus faecium (VRE strain ATCC 700221), Acinetobacter baumannii (ATCC 19606), Pseudomonas aeruginosa (PAO1), Klebsiella pneumonia (ATCC 13883) and Escherichia coli clinical isolate (UAEC-1). All compounds were stored as DMSO stocks at room temperature in the absence of light for several months at a time without observing any loss in biological activity. To ensure compound integrity of our DMSO stock solutions, we did not subject DMSO stocks of our test compounds to freeze-thaw cycles. 
2.) Synthetic Procedures and Characterization Data:<smiles>COc1cccc(O)c1O</smiles>

47

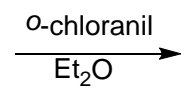

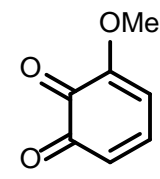

48

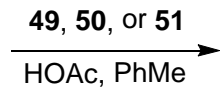

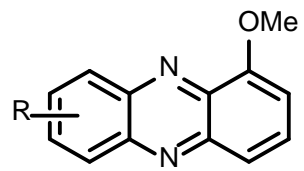

$20,30,31$

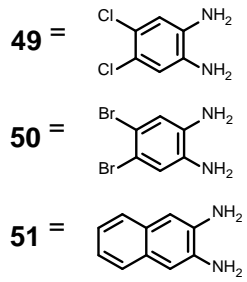

General condensation procedure for the synthesis of 1-methoxyphenazines (20, 30, 31): In a round-bottom flask, 3-methoxycatechol 47 (1.73 g, $12.3 \mathrm{mmol})$ was dissolved in diethyl ether $(35 \mathrm{~mL})$, then cooled to $-78{ }^{\circ} \mathrm{C}$ in a dry ice bath. Tetrachloro-o-benzoquinone ( $3.19 \mathrm{~g}, 12.5 \mathrm{mmol}$ ) was added and the reaction was stirred for 4 hours at a constant $-78{ }^{\circ} \mathrm{C}$. The reaction mixture was filtered twice under vacuum to afford benzoquinone $\mathbf{4 8}$ as a dark brown solid, which was used without further purification. Compound 3 was added to a $250 \mathrm{~mL}$ roundbottom flask containing 49 (1.29 g, $7.3 \mathrm{mmol}$ ) in glacial acetic acid (35 mL) and toluene (35 mL). The reaction was allowed to stir for 24 hours at room temperature. The mixture was neutralized with a solution of saturated sodium bicarbonate, washed with brine, and then extracted with dichloromethane. The combined organic layers were dried with sodium sulfate, filtered, and then removed in vacuo. The resulting crude solid was purified via column chromatography using dichloromethane as the eluent to afford pure compound 20 as a yellow solid (86\%, 1.49 g). Note: Analogous procedures were used to synthesize $\mathbf{3 0}$ and $\mathbf{3 1 .}$

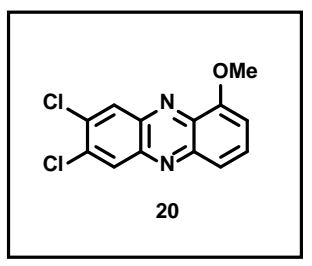

Yield: $86 \%$ yield; $1.49 \mathrm{~g}$ of $\mathbf{2 0}$ was isolated as a yellow solid.

${ }^{1} \mathbf{H}$ NMR (400 MHz, CDCl $)$ ): $\delta 8.53$ (s, 1H), 8.35 (s, 1H), $7.80-7.76$ (m, 2H), 7.09 (dd, J $=4.3,4.3 \mathrm{~Hz}, 1 \mathrm{H}), 4.17(\mathrm{~s}, 3 \mathrm{H})$.

${ }^{13} \mathrm{C}$ NMR (100 MHz, $\left.\mathbf{C D C l}_{3}\right): \delta$ 155.3, 144.8, 142.2, 140.8, 137.4, 136.0, 135.4, 131.7, 130.5, 129.8, 121.6, 107.4, 56.8.

MP: 253 - $255^{\circ} \mathrm{C}$, lit. 245 - $247^{\circ} \mathrm{C} .{ }^{1}$

Note: NMR spectra match those previously reported. ${ }^{1}$

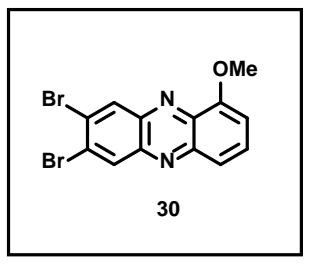

Yield: $78 \%$ yield; $1.09 \mathrm{~g}$ of $\mathbf{3 0}$ was isolated as a yellow solid.

${ }^{1} \mathbf{H}$ NMR (400 MHz, $\left.\mathbf{C D C l}_{3}\right): \delta 8.72(\mathrm{~s}, 1 \mathrm{H}), 8.54(\mathrm{~s}, 1 \mathrm{H}), 7.81-7.73(\mathrm{~m}, 2 \mathrm{H}), 7.08$ (dd, $J$ $=6.0,2.7 \mathrm{~Hz}, 1 \mathrm{H}), 4.16(\mathrm{~s}, 3 \mathrm{H})$.

${ }^{13}$ C NMR (100 MHz, $\left.\mathbf{C D C l}_{3}\right): \delta$ 155.3, 144.8, 142.5, 141.1, 137.4, 134.0, 133.3, 131.8, 128.1, 127.4, 121.6, 107.5, 56.8.

HRMS (DART): calc. for $\mathrm{C}_{13} \mathrm{H}_{9} \mathrm{Br}_{2} \mathrm{~N}_{2} \mathrm{O}[\mathrm{M}+\mathrm{H}]^{+}$: 366.9076, found: 366.9081 .

MP: $231-233^{\circ} \mathrm{C}$.

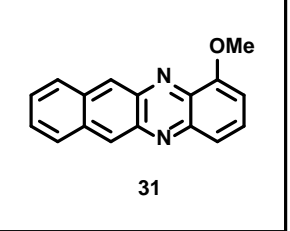

Yield: 76\% yield; $907 \mathrm{mg}$ of $\mathbf{3 1}$ was isolated as a red solid.

${ }^{1}$ H NMR (400 MHz, CDCl 3 ): $\delta 9.08$ (s, 1H), 8.89 (s, 1H), 8.17 - 8.01 (m, 2H), 7.82 (dd, $J$ $=9.1,1.1 \mathrm{~Hz}, 1 \mathrm{H}), 7.73(\mathrm{dd}, J=9.1,7.4 \mathrm{~Hz}, 1 \mathrm{H}), 7.58-7.50$ (m, 2H), 7.01 (dd, $J=7.4,1.1$ $\mathrm{Hz}, 1 \mathrm{H}), 4.21$ (s, 3H).

MP: 223 - $225^{\circ} \mathrm{C}$., lit. $245-247^{\circ} \mathrm{C}$.

Note: ${ }^{1} \mathrm{H}$ NMR was identical to previously reported spectra. ${ }^{1}$ 


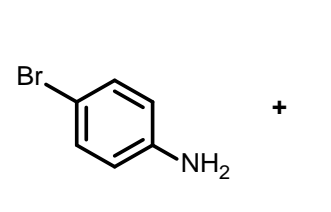

45

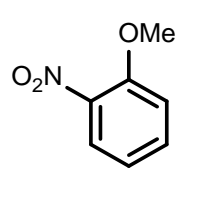

46

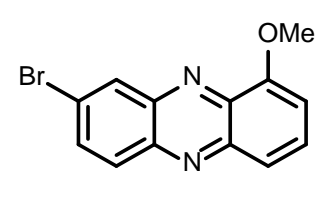

52

Synthesis of 7-bromo-1-methoxyphenazine (52): To a $100 \mathrm{~mL}$ round-bottom flask was added 4-bromoaniline 45 (1.74 mL, $14.2 \mathrm{mmol}$ ), 2-nitroanisole 46 (2.45 g, $14.2 \mathrm{mmol}$ ), and potassium hydroxide (3.99 g, $71.1 \mathrm{mmol})$ in toluene $(20 \mathrm{~mL})$. The reaction was then allowed to reflux for 24 hours. After the reaction was complete, the reaction contents were allowed to cool to room temperature. The reaction mixture was transferred to a separatory funnel, partitioned between ethyl acetate, and then washed with water and brine. The organic layers were collected, dried with anhydrous sodium sulfate, filtered and then concentrated in vacuo. The resulting crude solid was purified via column chromatography using 99:1 to 85:15 hexanes:ethyl acetate to afford 52, which was isolated as a yellow solid (2\%, $87.7 \mathrm{mg})$.

${ }^{1} \mathbf{H}$ NMR (400 MHz, $\left.\mathbf{C D C l}_{3}\right): \delta 8.58(\mathrm{~d}, J=2.2 \mathrm{~Hz}, 1 \mathrm{H}), 8.05(\mathrm{~d}, J=9.2 \mathrm{~Hz}, 1 \mathrm{H}), 7.86$ (dd, $J=9.2,2.2 \mathrm{~Hz}$, $1 \mathrm{H}), 7.80-7.70$ (m, 2H), 7.05 (dd, $J=7.1,1.5 \mathrm{~Hz}, 1 \mathrm{H}), 4.15$ (s, 3H).

${ }^{13}$ C NMR (100 MHz, CDCl 3$): \delta 155.2,144.4,142.5,142.2,137.2,134.7,132.3,131.1,130.8,124.7,121.6$, 107.3, 56.7 .

HRMS (ESI): calc. for $\mathrm{C}_{13} \mathrm{H}_{10} \mathrm{BrN}_{2} \mathrm{O}[\mathrm{M}+\mathrm{H}]^{+}:$288.9971, found: 288.9960 .

MP: 194 - $196{ }^{\circ} \mathrm{C}$., lit. 187 - $188^{\circ} \mathrm{C} .^{2}$

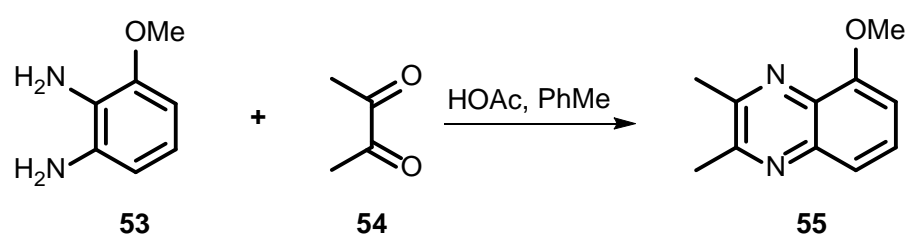

Synthesis of 5-methoxy-2,3,-dimethylquinoxaline (55): 2,3-Diaminoanisole 53 (300 mg, $2.17 \mathrm{mmol}$ ) was added to a $100 \mathrm{~mL}$ round-bottom flask containing glacial acetic acid (18 mL), toluene (22 mL), and 2,3butanedione $54(190 \mu \mathrm{L}, 2.17 \mathrm{mmol})$. The reaction was then left to stir for 21 hours at room temperature. Upon completion of the reaction, the resulting mixture was neutralized with an aqueous solution of saturated sodium bicarbonate, washed with brine, and extracted with dichloromethane. The resulting organic layers were dried with sodium sulfate, filtered, and concentrated in vacuo. The resulting crude material was purified via column chromatography using 99:1 to 97:3 dichloromethane:methanol to afford 55 in 90\% yield (367 mg) as an offwhite solid. Note: 2,3-Diaminoanisole was purchased as the dihydrochloride salt, which was treated with a solution of saturated aqueous sodium bicarbonate in a separatory funnel, and then extracted with ethyl acetate to afford the free-base 53, which was used in the reaction. This procedure was modified from a published reaction. $^{1}$

${ }^{1} \mathbf{H}$ NMR (400 MHz, $\left.\mathbf{C D C l}_{3}\right): \delta 7.45-7.39$ (m, 2H), 6.86 (dd, $\left.J=5.8,3.2 \mathrm{~Hz}, 1 \mathrm{H}\right), 3.94$ (s, 3H), 2.63 (s, 3H), $2.58(\mathrm{~s}, 3 \mathrm{H})$.

${ }^{13}$ C NMR (100 MHz, CDCl 3$): \delta 154.5,153.7,152.1,141.9,132.9,128.6,120.1,107.1,56.1,23.3,23.1$.

Note: NMR spectra match those previously reported. ${ }^{1}$ 


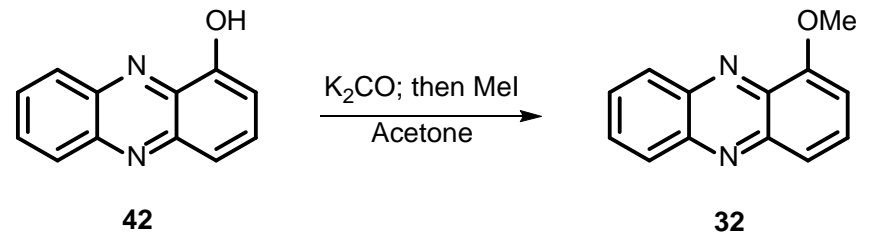

Synthesis of 1-methoxyphenazine (32): Potassium carbonate (2.26 g, $16.3 \mathrm{mmol})$ was added to a stirring solution of 1-hydroxyphenazine 42 (643 mg, $3.26 \mathrm{mmol}$ ) in $20 \mathrm{~mL}$ acetone. The resulting mixture was allowed to stir at room temperature for 30 minutes before iodomethane $(2.03 \mathrm{~mL}, 32.6 \mathrm{mmol})$ was added to the reaction. The resulting reaction mixture was allowed to stir for an additional 17 hours. The reaction contents were transferred to a separatory funnel and extracted with ethyl acetate. The organic contents were combined, dried with sodium sulfate, and then removed in vacuo to afford 32 in >99\% yield (683 mg) as a yellow solid.

${ }^{1}$ H NMR (400 MHz, CDCl $)$ ): $\delta 8.40(\mathrm{~m}, 1 \mathrm{H}), 8.22(\mathrm{~m}, 1 \mathrm{H}), 7.87-7.79(\mathrm{~m}, 2 \mathrm{H}), 7.83(\mathrm{~d}, J=8.9 \mathrm{~Hz}, 1 \mathrm{H}), 7.75$ (dd, $J=8.9,7.5 \mathrm{~Hz}, 1 \mathrm{H}$ ), 7.07 (dd, $J=7.5,1.4 \mathrm{~Hz}, 1 \mathrm{H}$ ), 4.17 (s, 3H).

MP: 166 - $168^{\circ} \mathrm{C}$., lit. $166-168^{\circ} \mathrm{C} .{ }^{1}$

Note: ${ }^{1} \mathrm{H}$ NMR spectrum and melting point match those previously reported. ${ }^{1}$

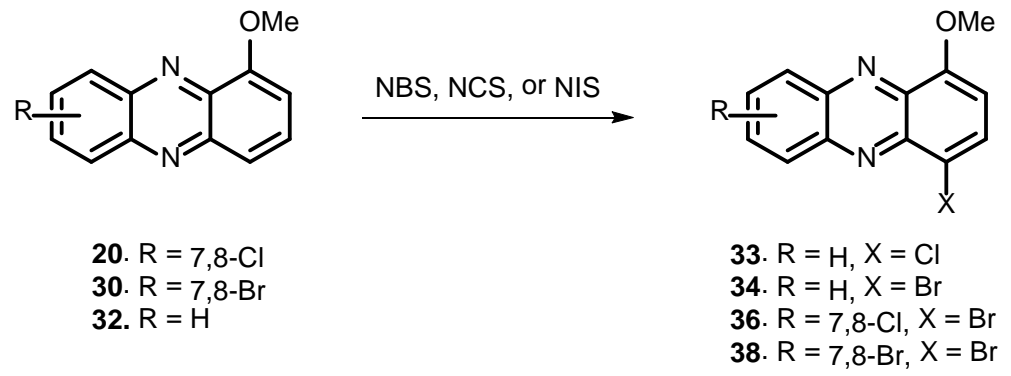

General procedure for mono-halogenation of the 4-position of 1-methoxyphenazines (33, 34, 36, 38): 7,8Dichloro-1-methoxyphenazine 20 (151 mg, $0.54 \mathrm{mmol}$ ) was dissolved in dichloromethane (15 mL) before $N$ bromosuccinimide (106 $\mathrm{mg}, 0.60 \mathrm{mmol}$ ) was added and the reaction was brought to reflux. The mixture was left to stir overnight until complete (monitored by TLC with dichloromethane). At this time, the reaction was concentrated and adsorbed onto silica gel (via dissolving the crude reaction contents and silica gel in dichloromethane, then concentrating via rotavap) and purified via column chromatography using dichloromethane to elute pure 4-bromo-7,8-dichloro-1-methoxyphenazine 36, which was isolated as a dark yellow solid (96\%, $185 \mathrm{mg})$. Notes: Analogous procedures were used for the chlorination and iodination of 1methoxyphenazines using $N$-chlorosuccinimide or $N$-iodosuccinimide respectively. We observed the formation of side products 33a and 33b during the synthesis of 33. The synthesis of $\mathbf{3 8}$ yielded demethylated and orthobrominated side product $\mathbf{1 7}$ in trace amounts. Compound $\mathbf{3 4}$ has been previously reported. ${ }^{1}$

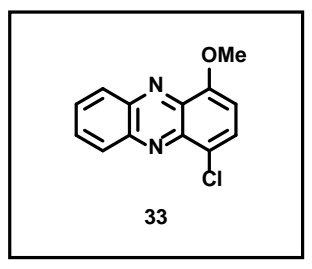

Yield: $76 \%$ yield; $280 \mathrm{mg}$ of $\mathbf{3 3}$ was isolated as a yellow solid.

${ }^{1} \mathbf{H}$ NMR (400 MHz, $\left.\mathbf{C D C l}_{3}\right): \delta 8.19-8.08$ (m, 2H), $7.71-7.61$ (m, 2H), 7.53 (d, $J=8.2$ $\mathrm{Hz}, 1 \mathrm{H}), 6.66$ (d, $J=8.2 \mathrm{~Hz}, 1 \mathrm{H}), 3.90$ (s, 3H).

${ }^{13}$ C NMR (100 MHz, $\left.\mathbf{C D C l}_{3}\right): \delta$ 154.0, 142.9, 141.8, 139.9, 136.6, 131.1, 130.8, 129.6, 129.5, 129.2, 123.5, 105.7, 56.3.

HRMS (DART): calc. for $\mathrm{C}_{13} \mathrm{H}_{10} \mathrm{ClN}_{2} \mathrm{O}[\mathrm{M}+\mathrm{H}]^{+}:$245.0476, found: 245.0484 .

MP: $149-151^{\circ} \mathrm{C}$. 


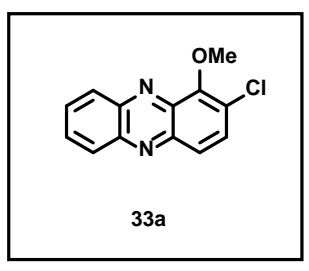

HRMS (DART): calc. for $\mathrm{C}_{13} \mathrm{H}_{10} \mathrm{ClN}_{2} \mathrm{O}[\mathrm{M}+\mathrm{H}]^{+}$: 245.0476, found: 245.0479 .

MP: $139-141^{\circ} \mathrm{C}$.

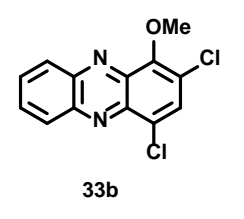

Yield: 6\% yield; $42.6 \mathrm{mg}$ of $\mathbf{3 3 b}$ was isolated as a yellow solid.

${ }^{1}$ H NMR (400 MHz, CDCl 3 ): $\delta$ 8.37-8.30 (m, 2H), 7.93 (s, 1H), 7.91-7.87 (m, 2H), 4.30 (s, 3H).

${ }^{13}$ C NMR (100 MHz, CDCl $): \delta$ 151.0, 143.0, 142.9, 139.6, 139.3, 132.0, 131.7, 131.1, 130.1, 130.0, 128.6, 126.2, 62.9.

HRMS (DART): calc. for $\mathrm{C}_{13} \mathrm{H}_{9} \mathrm{Cl}_{2} \mathrm{~N}_{2} \mathrm{O}[\mathrm{M}+\mathrm{H}]^{+}: 279.0086$, found: 279.0097 .

MP: $182-184^{\circ} \mathrm{C}$.

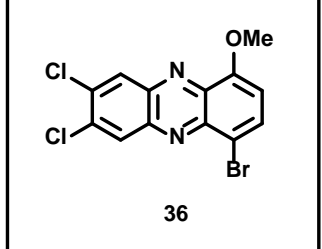

Yield: 96\% yield; $185 \mathrm{mg}$ of $\mathbf{3 6}$ was isolated as a dark yellow solid.

${ }^{1}$ H NMR (400 MHz, CDCl $\left.\mathbf{~ N}_{3}\right): \delta 8.56(\mathrm{~s}, 1 \mathrm{H}), 8.54(\mathrm{~s}, 1 \mathrm{H}), 8.11(\mathrm{~d}, J=8.3 \mathrm{~Hz}, 1 \mathrm{H}), 6.99$ (d, $J=8.3 \mathrm{~Hz}, 1 \mathrm{H}), 4.17$ (s, 3H).

${ }^{13} \mathrm{C}$ NMR (100 MHz, $\left.\mathbf{C D C l}_{3}\right): \delta 155.3,142.2,141.6,140.9,137.8,136.8,136.4,134.4$, 130.2, 130.2, 114.3, 107.9, 57.0.

HRMS (DART): calc. for $\mathrm{C}_{13} \mathrm{H}_{8} \mathrm{BrCl}_{2} \mathrm{~N}_{2} \mathrm{O}[\mathrm{M}+\mathrm{H}]^{+}$: 356.9192, found: 356.9194.

MP: $199-201^{\circ} \mathrm{C}$.

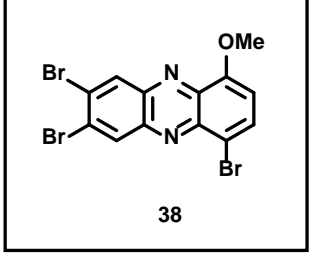

Yield: $86 \%$ yield; $275 \mathrm{mg}$ of $\mathbf{3 8}$ was isolated as a dark yellow solid.

${ }^{1}$ H NMR (400 MHz, CDCl $\left.\mathbf{~ M}_{3}\right): \delta .75(\mathrm{~s}, 1 \mathrm{H}), 8.72(\mathrm{~s}, 1 \mathrm{H}), 8.10$ (d, $\left.J=8.3 \mathrm{~Hz}, 1 \mathrm{H}\right), 6.98$ (d, $J=8.3 \mathrm{~Hz}, 1 \mathrm{H}), 4.16(\mathrm{~s}, 3 \mathrm{H})$.

${ }^{13}$ C NMR (100 MHz, CDCl 3$): \delta 155.3,142.5,141.6,141.2,137.8,134.5,133.7,133.6$, 129.1, 128.6, 114.4, 107.9, 57.0.

HRMS (DART): calc. for $\mathrm{C}_{13} \mathrm{H}_{8} \mathrm{Br}_{3} \mathrm{~N}_{2} \mathrm{O}[\mathrm{M}+\mathrm{H}]^{+}$: 444.8181 , found: 444.8181 .

MP: $241-243^{\circ} \mathrm{C}$.

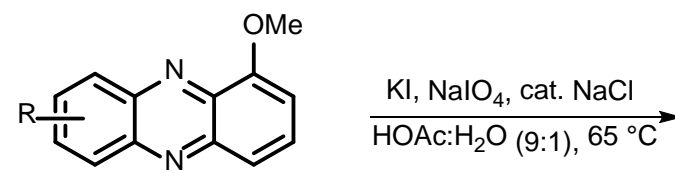

32. $\mathrm{R}=7,8-\mathrm{H}$

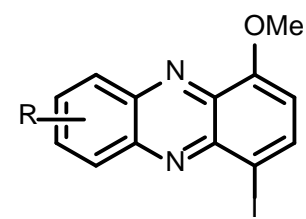

35. $\mathrm{R}=7,8-\mathrm{H}$

Synthesis of 4-iodo-1-methoxyphenazine (35): To a mixture of 1-methoxyphenazine 32 (356 mg, $1.69 \mathrm{mmol}$ ) in $30 \mathrm{~mL}$ of acetic acid:water (9:1) was added sodium chloride (435 mg, $7.45 \mathrm{mmol}$ ), sodium periodate (869 
$\mathrm{mg}, 4.06 \mathrm{mmol})$, then potassium iodide $(675 \mathrm{mg}, 4.06 \mathrm{mmol})$. The reaction was heated to $65{ }^{\circ} \mathrm{C}$ and allowed to stir for 21 hours. After completion of this reaction as determined by TLC analysis (with dichloromethane), the reaction mixture was washed with a saturated solution of sodium bicarbonate and then partitioned with dichloromethane. The organic layers were then collected, dried with anhydrous sodium sulfate and concentrated in vacuo. The resulting solid was then dry loaded onto silica gel (using dichloromethane) and purified via column chromatography using first hexanes to elute purple side product, followed by dichloromethane to elute compound 35, which was isolated as a yellow solid (68\%, $388 \mathrm{mg})$. Note: This is a modified protocol from a previously published procedure. ${ }^{3}$

${ }^{1} \mathbf{H}$ NMR (400 MHz, $\left.\mathbf{C D C l}_{3}\right): \delta 8.37(\mathrm{~m}, 1 \mathrm{H}), 8.32(\mathrm{~m}, 1 \mathrm{H}), 8.30(\mathrm{~d}, J=8.2 \mathrm{~Hz}, 1 \mathrm{H}), 7.89-7.79(\mathrm{~m}, 2 \mathrm{H}), 6.81$ (d, $J=8.2 \mathrm{~Hz}, 1 \mathrm{H}), 4.12$ (s, 3H).

${ }^{13}$ C NMR (100 MHz, $\left.\mathbf{C D C l}_{3}\right): \delta$ 156.2, 144.1, 142.6, 140.2, 136.9, 131.4, 131.1, 129.8, 129.7, 108.2, 91.0, 56.8. Note: One signal obscured likely due to overlap.

HRMS (DART): calc. for $\mathrm{C}_{13} \mathrm{H}_{10} \mathrm{IN}_{2} \mathrm{O}[\mathrm{M}+\mathrm{H}]^{+}$: 336.9832, found: 336.9829 .

MP: $169-171^{\circ} \mathrm{C}$.

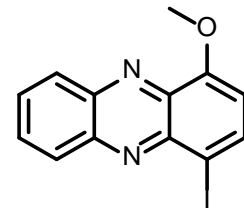

35

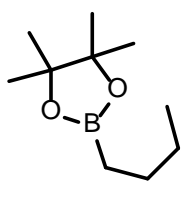

64

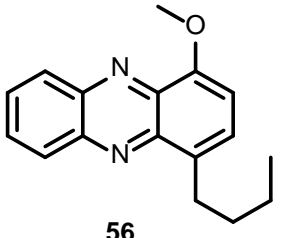

56

Synthesis of 4-butyl-1-methoxyphenazine (56): To a $50 \mathrm{~mL}$ round-bottom flask was added 4iodomethoxyphenazine 35 (146 mg, $0.43 \mathrm{mmol}$ ), tetrakis(triphenylphosphine)palladium(0) (100 mg, $0.1 \mathrm{mmol}$ ), sodium hydroxide (347 mg, $8.67 \mathrm{mmol}$ ), and $n$-butylboronic acid pinacol ester 64 (1.43 mL, $6.72 \mathrm{mmol})$ in 6 $\mathrm{mL}$ of a toluene:water $(2: 1)$ solution. The mixture was brought to $90{ }^{\circ} \mathrm{C}$ in an oil bath and left to stir for 48 hours. After cooling to room temperature, the reaction mixture was transferred to a separatory funnel, partitioned between an aqueous solution of saturated sodium bicarbonate and ethyl acetate. The resulting organic layer was washed with a $1 \mathrm{~N}$ solution of hydrochloric acid, and then brine. The organic layers were combined and dried with anhydrous sodium sulfate and concentrated in vacuo. The resulting crude material was purified via column chromatography using 99:1 to 85:15 hexanes:ethyl acetate to afford $\mathbf{5 6}$ as a yellow solid (34\% yield, $38.9 \mathrm{mg}$ ).

${ }^{1} \mathbf{H}$ NMR (400 MHz, $\left.\mathbf{C D C l}_{3}\right): \delta 8.38(\mathrm{~m}, 1 \mathrm{H}), 8.26(\mathrm{~m}, 1 \mathrm{H}), 7.85-7.78(\mathrm{~m}, 2 \mathrm{H}), 7.54(\mathrm{dt}, J=7.8,0.9 \mathrm{~Hz}, 1 \mathrm{H})$, 7.00 (d, $J=7.8 \mathrm{~Hz}, 1 \mathrm{H}), 4.15$ (s, 3H), 3.30 (td, $J=7.6,0.9 \mathrm{~Hz}, 2 \mathrm{H}), 1.87-1.72$ (m, 2H), 1.47 (tq, $J=7.4,7.4$ $\mathrm{Hz}, 2 \mathrm{H}), 0.99$ (t, $J=7.4 \mathrm{~Hz}, 3 \mathrm{H})$.

${ }^{13}$ C NMR (100 MHz, CDCl 3 ): $\delta$ 153.4, 143.3, 143.0, 141.9, 137.2, 133.9, 130.4, 130.2, 130.1, 130.1, 128.4, 106.5, 56.5, 32.9, 30.6, 22.9, 14.3 .

HRMS (ESI): calc. for $\mathrm{C}_{17} \mathrm{H}_{19} \mathrm{~N}_{2} \mathrm{O}[\mathrm{M}+\mathrm{H}]^{+}:$267.1492, found: 267.1497.

MP: $77-79{ }^{\circ} \mathrm{C}$. 


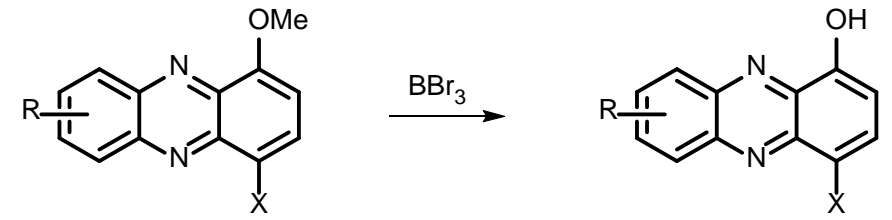

20. $\mathrm{R}=7,8-\mathrm{Cl}, X=\mathrm{H}$

30. $\mathrm{R}=7,8-\mathrm{Br}, X=\mathrm{H}$

31. $\mathrm{R}=\mathrm{Ph}, \mathrm{X}=\mathrm{H}$

33. $R=H, X=B r$

34. $\mathrm{R}=\mathrm{H}, \mathrm{X}=\mathrm{Cl}$

35. $\mathrm{R}=\mathrm{H}, \mathrm{X}=\mathrm{I}$

36. $\mathrm{R}=7,8-\mathrm{Cl}, \mathrm{X}=\mathrm{Br}$

37. $\mathrm{R}=7,8-\mathrm{Cl}, \mathrm{X}=\mathrm{I}$

38. $\mathrm{R}=7,8-\mathrm{Br}, X=\mathrm{Br}$

52. $\mathrm{R}=8-\mathrm{Br}, \mathrm{X}=\mathrm{H}$

56. $\mathrm{R}=\mathrm{H}, \mathrm{X}=n$-Butyl
11. $\mathrm{R}=7,8-\mathrm{Cl}, \mathrm{X}=\mathrm{Br}$

12. $\mathrm{R}=7,8-\mathrm{Cl}, \mathrm{X}=\mathrm{I}$

13. $\mathrm{R}=7,8-\mathrm{Br}, X=\mathrm{Br}$

21. $\mathrm{R}=7,8-\mathrm{Cl}, \mathrm{X}=\mathrm{H}$

39. $\mathrm{R}=\mathrm{H}, \mathrm{X}=\mathrm{Br}$

40. $\mathrm{R}=\mathrm{H}, X=\mathrm{Cl}$

41. $R=H, X=I$

43. $\mathrm{R}=7,8-\mathrm{Br}, X=\mathrm{H}$

44. $\mathrm{R}=\mathrm{Ph}, \mathrm{X}=\mathrm{H}$

57. $\mathrm{R}=8-\mathrm{Br}, \mathrm{X}=\mathrm{H}$

58. $R=H, X=n$-Butyl

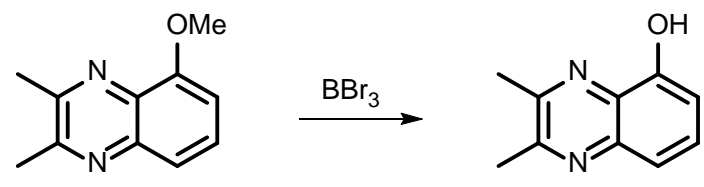

59

General demethylation procedure for the synthesis of 11, 13, 21, 40, 41, 57, 58 and 59: To a round bottom flask was added 7,8-dichloro-1-methoxyphenazine 20 (218 $\mathrm{mg}, 0.78 \mathrm{mmol}$ ) dissolved in anhydrous dichloromethane $(18 \mathrm{~mL})$. The mixture was brought to $-78{ }^{\circ} \mathrm{C}$ in a dry ice bath before dropwise addition of $1 \mathrm{M}$ boron tribromide solution in dichloromethane $(5.5 \mathrm{~mL}, 5.5 \mathrm{mmol})$. The reaction was left to stir at $-78{ }^{\circ} \mathrm{C}$ for 1 hour, and then allowed to reach ambient temperature for reaction overnight. The reaction was heated to reflux for 8 hours until complete (monitored by TLC). The solution was transferred to a separatory funnel containing an aqueous solution of saturated sodium bicarbonate, and then extracted with dichloromethane. Organic solvents were dried with sodium sulfate, filtered through cotton, and removed in vacuo. The resulting solid was purified via column chromatography using dichloromethane to elute compound $\mathbf{2 1}$ as an orange solid ( $>99 \%$, $209 \mathrm{mg}$ ). Note: Analogous procedures were used for all demethylation reactions using $\mathrm{BBr}_{3}$.

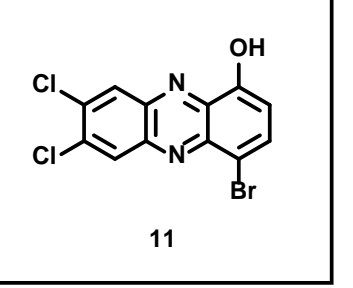

MP: $245-247^{\circ} \mathrm{C}$.

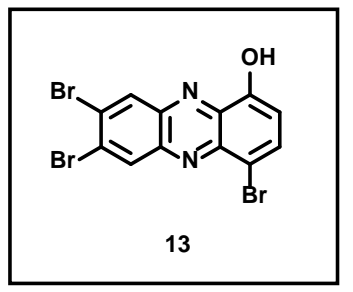

Yield: $91 \%$ yield; $145 \mathrm{mg}$ of $\mathbf{1 1}$ was isolated as an orange solid.

${ }^{1} \mathbf{H}$ NMR (400 MHz, $\left.\mathbf{C D C l}_{3}\right): \delta 8.58(\mathrm{~s}, 1 \mathrm{H}), 8.40(\mathrm{~s}, 1 \mathrm{H}), 8.12(\mathrm{~d}, J=8.1 \mathrm{~Hz}, 1 \mathrm{H})$, 8.04 (s, $1 \mathrm{H}), 7.17$ (d, $J=8.1 \mathrm{~Hz}, 1 \mathrm{H})$.

${ }^{13}$ C NMR (100 MHz, CDCl $): ~ \delta 151.8,142.9,141.4,139.9,136.9,135.6,135.6,130.5$, 129.2, 112.5, 110.6. Note: Absence of one ${ }^{13} \mathrm{C}$ signal likely due to peak overlap.

HRMS (DART): calc. for $\mathrm{C}_{12} \mathrm{H}_{6} \mathrm{BrCl}_{2} \mathrm{~N}_{2} \mathrm{O}[\mathrm{M}+\mathrm{H}]^{+}$: 342.9035, found: 342.9047.

Yield: $96 \%$ yield; $227 \mathrm{mg}$ of $\mathbf{1 3}$ was isolated as an orange solid.

${ }^{1}$ H NMR (400 MHz, CDCl $): \delta 8.80(\mathrm{~s}, 1 \mathrm{H}), 8.62(\mathrm{~s}, 1 \mathrm{H}), 8.12(\mathrm{~d}, J=8.1 \mathrm{~Hz}, 1 \mathrm{H}), 8.04$ (s, 1H), 7.18 (d, $J=8.1 \mathrm{~Hz}, 1 \mathrm{H})$.

${ }^{13}$ C NMR (100 MHz, $\left.\mathbf{C D C l}_{3}\right): \delta 151.8,143.2,141.4,140.2,135.8,135.6,134.0,132.7$, 129.7, 129.1, 112.5, 110.7. Note: Poor resolution due to insolubility in all solvents used

HRMS (DART): calc. for $\mathrm{C}_{12} \mathrm{H}_{6} \mathrm{Br}_{3} \mathrm{~N}_{2} \mathrm{O}[\mathrm{M}+\mathrm{H}]^{+}$: 430.8025, found: 430.8017 .

MP: $>260^{\circ} \mathrm{C}$. $\left(\mathrm{CDCl}_{3}, d_{6}\right.$-DMSO, $d_{6}$-Benzene, $d_{4}$-MeOD). 

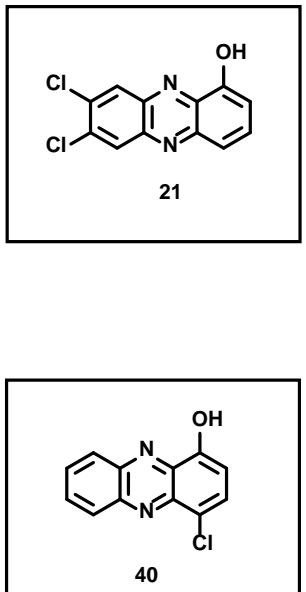

MP: $196-198^{\circ} \mathrm{C}$.

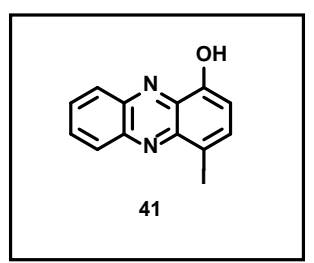

MP: $193-195^{\circ} \mathrm{C}$.

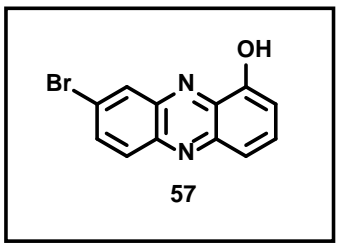

Yield: $86 \%$ yield; $299 \mathrm{mg}$ of $\mathbf{4 1}$ was isolated as a yellow solid.

${ }^{1}$ H NMR (400 MHz, CDCl $): \delta 8.41(\mathrm{~m}, 1 \mathrm{H}), 8.36(\mathrm{~d}, J=8.0 \mathrm{~Hz}, 1 \mathrm{H}), 8.30-8.20(\mathrm{~m}$, 2H), $7.97-7.82$ (m, 2H), 7.06 (d, $J=8.0 \mathrm{~Hz}, 1 \mathrm{H})$.

${ }^{13}$ C NMR (100 MHz, CDCl $): \delta$ 152.9, 144.9, 142.6, 141.6, 141.5, 134.9, 131.5, 131.5, 130.2, 128.9, 110.8, 88.4.

HRMS (DART): calc. for $\mathrm{C}_{12} \mathrm{H}_{8} \mathrm{IN}_{2} \mathrm{O}[\mathrm{M}+\mathrm{H}]^{+}$: 322.9676, found: 322.9689 .

Note: ${ }^{1} \mathrm{H}$ NMR and melting point match those previously reported. ${ }^{1}$

Yield: 95\% yield; $248 \mathrm{mg}$ of $\mathbf{4 0}$ was isolated as a yellow solid.

${ }^{1}$ H NMR (400 MHz, CDCl $\mathbf{~ M}_{3}: \delta 8.40(\mathrm{~m}, 1 \mathrm{H}), 8.23(\mathrm{~m}, 1 \mathrm{H}), 8.17$ (s, 1H), 7.97 - 7.86 (m, 2H), 7.87 (d, $J=8.2 \mathrm{~Hz}, 1 \mathrm{H}), 7.17$ (d, $J=8.2 \mathrm{~Hz}, 1 \mathrm{H})$.

${ }^{13}$ C NMR (100 MHz, CDCl 3$): \delta$ 151.1, 144.2, 141.4, 140.3, 135.1, 131.6, 131.1, 130.4, 129.1, 122.5, 108.8. Note: One ${ }^{13} \mathrm{C}$ signal missing, likely due to overlap.

HRMS (DART): calc. for $\mathrm{C}_{12} \mathrm{H}_{8} \mathrm{ClN}_{2} \mathrm{O}[\mathrm{M}+\mathrm{H}]^{+}:$231.0320, found: 231.0330.

Yield: >99\% yield; 83.5 mg of $\mathbf{5 7}$ was isolated as a yellow solid.

${ }^{1}$ H NMR (400 MHz, CDCl $): \delta 8.42(\mathrm{dd}, J=2.2,0.5 \mathrm{~Hz}, 1 \mathrm{H}), 8.12$ (dd, $J=9.2,0.5 \mathrm{~Hz}$, 1H), 8.09 (s, 1H), 7.90 (dd, $J=9.3,2.2 \mathrm{~Hz}, 1 \mathrm{H}$ ), $7.82-7.66$ (m, 2H), 7.26 (dd, $J=6.9$, $1.7 \mathrm{~Hz}, 1 \mathrm{H})$.

${ }^{13}$ C NMR (100 MHz, $\left.\mathbf{C D C l}_{3}\right): \delta 151.8,144.0,142.9,141.5,135.0,134.7,132.5,131.3$, 131.1, 125.2, 120.2, 109.9.

HRMS (ESI): calc. for $\mathrm{C}_{12} \mathrm{H}_{8} \mathrm{BrN}_{2} \mathrm{O}[\mathrm{M}+\mathrm{H}]^{+}:$274.9815, found: 274.9826 .

MP: 189 - $191^{\circ} \mathrm{C}$., lit. $176-177^{\circ} \mathrm{C} .^{2}$

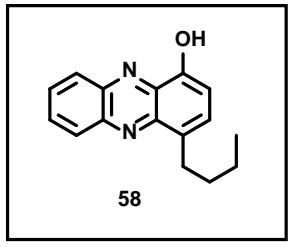

Yield: 90\% yield; $33.2 \mathrm{mg}$ of $\mathbf{5 8}$ was isolated as a yellow solid.

${ }^{1}$ H NMR (400 MHz, $\left.\mathbf{C D C l}_{3}\right): \delta 8.28$ (m, 1H), 8.17 (m, 1H), 8.10 (br. s, 1H), 7.84 - 7.76 (m, 2H), 7.53 (dt, $J=7.5,0.9 \mathrm{~Hz}, 1 \mathrm{H}), 7.15$ (d, $J=7.5 \mathrm{~Hz}, 1 \mathrm{H}), 3.28$ (td, $J=7.4 \mathrm{~Hz}, 0.9 \mathrm{~Hz}$, 2H), $1.84-1.75$ (m, 2H), 1.47 (tq, $J=7.4,7.4 \mathrm{~Hz}, 2 \mathrm{H}), 0.99$ (t, $J=7.4 \mathrm{~Hz}, 3 \mathrm{H})$.

${ }^{13} \mathrm{C}$ NMR (100 MHz, $\left.\mathbf{C D C l}_{3}\right): \delta$ 149.7, 143.5, 142.9, 140.8, 135.0, 132.6, 130.4, 130.4, 130.2, 129.6, 129.1, 108.7, 33.0, 30.3, 22.9, 14.3.

HRMS (ESI): calc. for $\mathrm{C}_{16} \mathrm{H}_{17} \mathrm{~N}_{2} \mathrm{O}[\mathrm{M}+\mathrm{H}]^{+}$: 253.1335, found: 253.1325.

MP: $138-140^{\circ} \mathrm{C}$. 


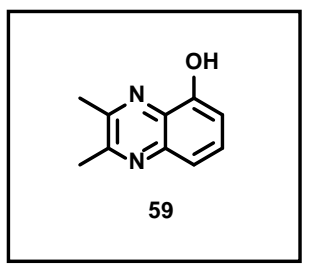

Yield: 86\% yield; 149 mg of $\mathbf{5 9}$ was isolated as an off-white solid.

${ }^{1} \mathbf{H}$ NMR (400 MHz, CDCl $)$ ): $\delta 7.85$ (br. s, $1 \mathrm{H}$ ), 7.55 (dd, $J=8.5,7.5 \mathrm{~Hz}, 1 \mathrm{H}$ ), 7.49 (dd, $J$ $=8.5,1.4 \mathrm{~Hz}, 1 \mathrm{H}), 7.11$ (dd, $J=7.5,1.4 \mathrm{~Hz}, 1 \mathrm{H}), 2.72$ (s, 3H), 2.69 (s, 3H).

${ }^{13} \mathrm{C}$ NMR (100 MHz, $\left.\mathbf{C D C l}_{3}\right): \delta 154.5,151.6,151.4,141.6,131.0,129.9,118.9,110.1$, 23.3, 23.1.

Note: NMR spectra match those previously reported. ${ }^{1}$

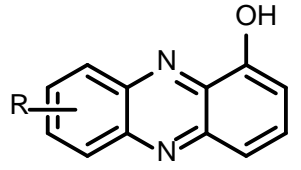

42. $\mathrm{R}=\mathrm{H}$

43. $\mathrm{R}=7,8-\mathrm{Br}$

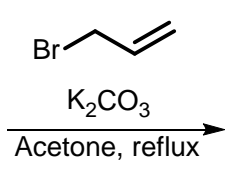

60. $\mathrm{R}=\mathrm{H}$

61. $\mathrm{R}=7,8-\mathrm{Br}$

General allylation procedure for the synthesis of 60 and 61: Potassium carbonate (866 mg, $6.27 \mathrm{mmol}$ ) was added to a stirring solution of 1-hydroxyphenazine 42 (247 mg, $1.25 \mathrm{mmol}$ ) in $15 \mathrm{~mL}$ acetone. The resulting mixture was allowed to stir at room temperature for 30 minutes before allyl bromide $(0.13 \mathrm{~mL}, 1.51 \mathrm{mmol})$ was added to the reaction. The resulting reaction mixture was allowed to stir for an additional 17 hours at reflux. The reaction contents were then transferred to a separatory funnel and extracted with ethyl acetate. The organic contents were then dried with anhydrous sodium sulfate and then removed in vacuo. The resulting crude solid was purified via column chromatography using 99:1 to 90:10 hexanes:ethyl acetate to afford 1allyloxyphenazines 60, which was isolated as a yellow solid (85\%, $251 \mathrm{mg})$.

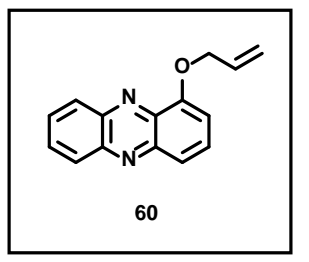

Yield: $85 \%$ yield; $251 \mathrm{mg}$ of $\mathbf{6 0}$ was isolated as a yellow solid.

${ }^{1}$ H NMR (100 MHz, CDCl $)$ ): $\delta 8.33(\mathrm{~m}, 1 \mathrm{H}), 8.15$ (m, 1H), $7.79-7.70$ (m, 3H), 7.63 (dd, $J=8.9,7.6 \mathrm{~Hz}, 1 \mathrm{H}), 6.99$ (d, $J=7.6 \mathrm{~Hz}, 1 \mathrm{H}), 6.17$ (ddt, $J=17.3,10.6,5.5 \mathrm{~Hz}, 1 \mathrm{H}), 5.47$ (ddt, $J=17.3,1.6,1.6 \mathrm{~Hz}, 1 \mathrm{H}), 5.32$ (ddt, $J=10.6,1.6,1.6 \mathrm{~Hz}, 1 \mathrm{H}), 4.88$ (ddd, $J=5.5,1.6$, $1.6 \mathrm{~Hz}, 2 \mathrm{H})$.

${ }^{13}$ C NMR (100 MHz, $\left.\mathbf{C D C l}_{3}\right): \delta$ 154.0, 144.3, 143.5, 142.3, 137.1, 132.7, 130.8, 130.4, 130.4, 130.1, 129.3, 121.6, 118.6, 108.2, 70.2.

HRMS (ESI): calc. for $\mathrm{C}_{15} \mathrm{H}_{13} \mathrm{~N}_{2} \mathrm{O}[\mathrm{M}+\mathrm{H}]^{+}$: 237.1022, found: 237.1026.

MP: $117-119^{\circ} \mathrm{C}$.

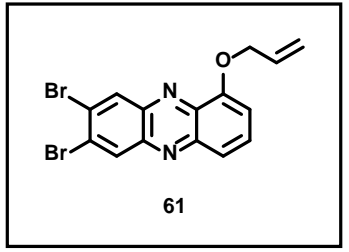

Yield: $88 \%$ yield; $109 \mathrm{mg}$ of $\mathbf{6 1}$ was isolated as an orange solid.

${ }^{1}$ H NMR (400 MHz, $\left.\mathbf{C D C l}_{3}\right): \delta 8.70$ (s, 1H), 8.51 (s, 1H), 7.76 - 7.69 (m, 2H), 7.07 (dd, $J=5.9,2.8 \mathrm{~Hz}, 1 \mathrm{H}$ ), 6.21 (ddt, $J=17.4,10.6,5.4 \mathrm{~Hz}, 1 \mathrm{H}$ ), 5.53 (ddt, $J=17.4,1.5,1.5$ Hz, 1H), 5.39 (ddt, $J=10.6,1.5,1.3 \mathrm{~Hz}, 1 \mathrm{H}$ ), 4.92 (ddd, $J=5.4,1.5,1.3 \mathrm{~Hz}, 2 \mathrm{H}$ ).

${ }^{13} \mathrm{C}$ NMR (100 MHz, $\left.\mathbf{C D C l}_{3}\right): \delta$ 154.2, 144.8, 142.4, 141.2, 137.6, 134.1, 133.2, 132.6, 131.7, 128.0, 127.2, 121.7, 119.0, 109.1, 70.4.

HRMS (ESI): calc. for $\mathrm{C}_{15} \mathrm{H}_{10} \mathrm{Br}_{2} \mathrm{~N}_{2} \mathrm{O}[\mathrm{M}+\mathrm{H}]^{+}$: 394.9213, found: 394.9211.

MP: $170-172{ }^{\circ} \mathrm{C}$. 


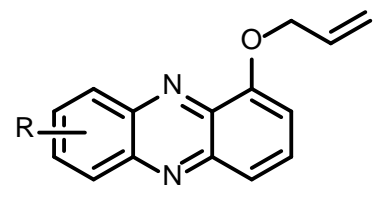

60. $\mathrm{R}=\mathrm{H}$

61. $\mathrm{R}=7,8-\mathrm{Br}$

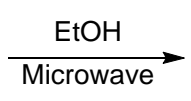

63. $\mathrm{R}=7,8-\mathrm{Br}$

General procedure for the synthesis of 62 and 63: To an $8 \mathrm{~mL}$ sealed microwave vial was added 1(allyloxy)phenazine $\mathbf{6 0}$ (19.4 mg, $0.08 \mathrm{mmol}$ ) in ethanol (4 mL). The resulting mixture was then heated at 150 ${ }^{\circ} \mathrm{C}$ in the microwave reactor for 90 minutes. The solvent was removed in vacuo and the resulting solid was purified via column chromatography using dichloromethane to elute, affording 2-allylphenazine 62, which was isolated as a yellow solid (>99\%, $19.3 \mathrm{mg})$. Note: Attempts were made to conduct this reaction under traditional thermal heating; however, reaction times exceeded 5 days and resulted in poor yields.

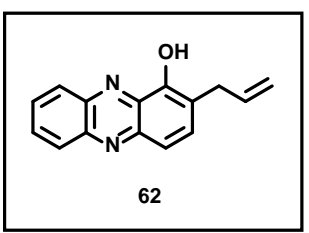

Yield: $>99 \%$ yield; $19.3 \mathrm{mg}$ of $\mathbf{6 2}$ was isolated as a yellow solid.

${ }^{1} \mathbf{H}$ NMR (100 MHz, $\left.\mathbf{C D C l}_{3}\right): \delta 8.28$ (br. s, 1H), 8.21 (m, 1H), 8.13 (m, 1H), $7.81-7.74$ (m, 2H), 7.72 (d, $J=9.1 \mathrm{~Hz}, 1 \mathrm{H}), 7.64$ (d, $J=9.1 \mathrm{~Hz}, 1 \mathrm{H}), 6.09$ (ddt, $J=16.7,10.1,6.6$ $\mathrm{Hz}, 1 \mathrm{H}$ ), 5.18 (ddt, $J=16.7,1.7,1.7 \mathrm{~Hz}, 1 \mathrm{H}$ ), 5.14 (ddt, $J=10.1,1.7 \mathrm{~Hz}, 1.6 \mathrm{~Hz}, 1 \mathrm{H}) 3.68$ (ddd, $J=6.7,1.7,1.6 \mathrm{~Hz}, 2 \mathrm{H}$ ).

${ }^{13}$ C NMR (100 MHz, CDCl $): \delta$ 148.2, 143.8, 143.0, 141.3, 136.0, 134.6, 134.5, 130.4, 130.4, 129.9, 129.2, 120.8, 119.6, 116.5, 34.0.

HRMS (ESI): calc. for $\mathrm{C}_{15} \mathrm{H}_{13} \mathrm{~N}_{2} \mathrm{O}[\mathrm{M}+\mathrm{H}]^{+}:$237.1022, found: 237.1017.

MP: $112-114^{\circ} \mathrm{C}$.

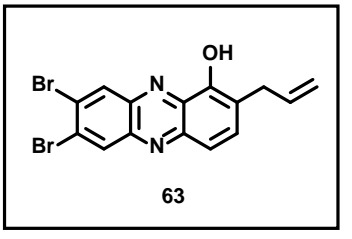

Yield: $>99 \%$ yield; $39.5 \mathrm{mg}$ of $\mathbf{6 3}$ was isolated as an orange solid.

${ }^{1} \mathbf{H}$ NMR (400 MHz, $\left.\mathbf{C D C l}_{3}\right): \delta 8.59$ (d, $\left.J=0.4 \mathrm{~Hz}, 1 \mathrm{H}\right), 8.56$ (d, $\left.J=0.4 \mathrm{~Hz}, 1 \mathrm{H}\right), 8.07$ (s, 1H), $7.74-7.69$ (m, 2H), 6.07 (ddt, $J=17.1,10.1,6.6 \mathrm{~Hz}, 1 \mathrm{H}$ ), 5.19 (ddt, $J=17.1$, 1.6, $1.6 \mathrm{~Hz}, 1 \mathrm{H}$ ), 5.16 (ddt, $J=10.1,1.6,1.5 \mathrm{~Hz}, 1 \mathrm{H}$ ) 3.69 (ddd, $J=6.6,1.6,1.5 \mathrm{~Hz}$, $2 \mathrm{H})$.

${ }^{13}$ C NMR (100 MHz, CDCl 3 ): $\delta 148.3,143.5,142.7,140.2,135.7,135.6,135.0,133.7,133.0,127.7,127.6$, 122.2, 119.9, 116.8, 34.1.

HRMS (ESI): calc. for $\mathrm{C}_{15} \mathrm{H}_{10} \mathrm{Br}_{2} \mathrm{~N}_{2} \mathrm{O}[\mathrm{M}+\mathrm{H}]^{+}$: 394.9213, found: 394.9216 .

MP: $199-201{ }^{\circ} \mathrm{C}$.

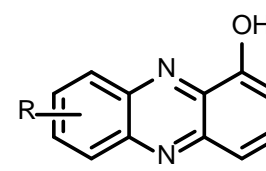

21. $\mathrm{R}=7,8-\mathrm{Cl}$ 42*. $\mathrm{R}=\mathrm{H}$

43. $\mathrm{R}=7,8-\mathrm{Br}$

44. $\mathrm{R}=\mathrm{Ph}$

57. $\mathrm{R}=8-\mathrm{Br}$
NBS or NCS

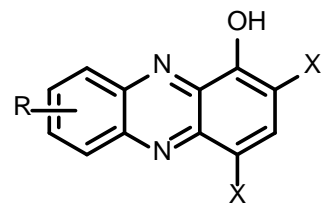

3. $\mathrm{R}=\mathrm{H}, \mathrm{X}=\mathrm{Cl}$

16. $\mathrm{R}=7,8-\mathrm{Cl}, \mathrm{X}=\mathrm{Br}$

17. $\mathrm{R}=7,8-\mathrm{Br}, \mathrm{X}=\mathrm{Br}$

18. $\mathrm{R}=8-\mathrm{Br}, \mathrm{X}=\mathrm{Br}$

19. $R=P h, X=B r$

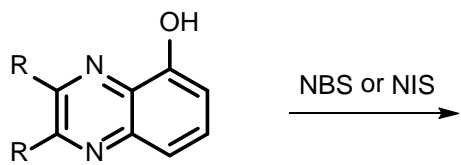

59. $\mathrm{R}=\mathrm{Me}$

63*. $\mathrm{R}=\mathrm{H}$

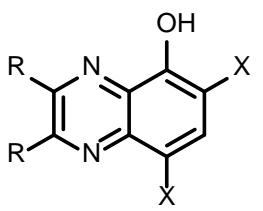

25. $R=H, X=B r$

26. $R=H, X=I$

27. $\mathrm{R}=\mathrm{Me}, \mathrm{X}=\mathrm{Br}$

28. $R=M e, X=I$ 
General dihalogenation procedure for synthesis of 3, 18 and 25-28: 7,8-Dibromo-1-hydroxyphenazine 43 (87.5 mg, $0.24 \mathrm{mmol}$ ) and $N$-bromosuccinimide, ( $86.2 \mathrm{mg}, 0.48 \mathrm{mmol}$ ) were dissolved in dichloromethane (15 $\mathrm{mL}$ ) and allowed to stir at room temperature for 2 hours. The reaction contents were then concentrated, adsorbed onto silica gel and purified via column chromatography using dichloromethane to elute 2,4,7,8tetrabromo-1-hydroxyphenazine 17, which was isolated as a dark orange solid (84\%, $104 \mathrm{mg})$.

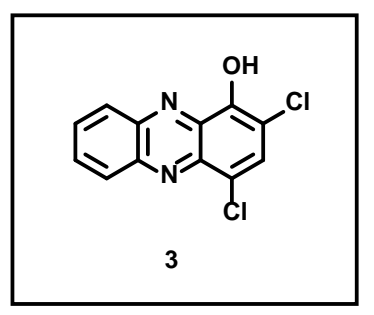

MP: $205-207^{\circ} \mathrm{C}$.

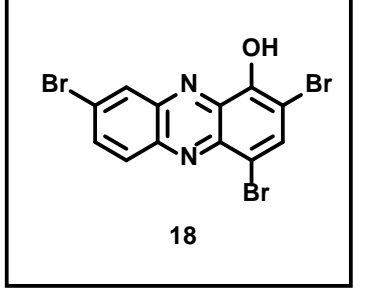

MP: $239-241^{\circ} \mathrm{C}$.
Yield: 47\% yield; $124 \mathrm{mg}$ of 3 was isolated as a yellow solid.

${ }^{1}$ H NMR (400 MHz, CDCl $)$ ): $\delta 8.40$ (m, 1H), 8.36 (br. s, 1H), 8.26 (m, 1H), 7.96-7.89 (m, 2H), 7.91 (s, 1H).

${ }^{13}$ C NMR (100 MHz, $\left.\mathbf{C D C l}_{3}\right): \delta 147.0,143.9,141.7,139.0,134.6,132.3,132.0,131.9$, 130.4, 129.1, 123.2, 114.6.

HRMS (DART): calc. for $\mathrm{C}_{12} \mathrm{H}_{7} \mathrm{~N}_{2} \mathrm{Cl}_{2} \mathrm{O}[\mathrm{M}+\mathrm{H}]^{+}$: 264.9930, found: 264.9932.

Yield: $46 \%$ yield; $31.3 \mathrm{mg}$ of $\mathbf{1 8}$ was isolated as an orange solid.

${ }^{1}$ H NMR (400 MHz, d6-DMSO): $\delta 8.50$ (d, $\left.J=2.2 \mathrm{~Hz}, 1 \mathrm{H}\right), 8.45$ (s, 1H), 8.26 (d, $J=$ $9.3 \mathrm{~Hz}, 1 \mathrm{H}$ ), 8.15 (dd, $J=9.3,2.2 \mathrm{~Hz}, 1 \mathrm{H}$ ).

${ }^{13}$ C NMR (100 MHz, d6-DMSO): $\delta$ 150.9, 141.5, 141.5, 139.6, 137.3, 135.8, 135.3, 131.3, 130.6, 125.7, 111.6, 105.3.

HRMS (ESI): calc. for $\mathrm{C}_{12} \mathrm{H}_{6} \mathrm{Br}_{3} \mathrm{~N}_{2} \mathrm{O}[\mathrm{M}+\mathrm{H}]^{+}$: 430.8025 , found: 430.8010 .

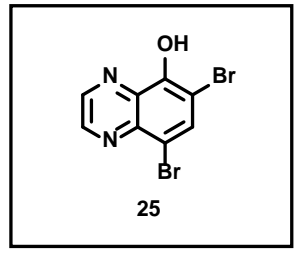

MP: $227-229^{\circ} \mathrm{C}$

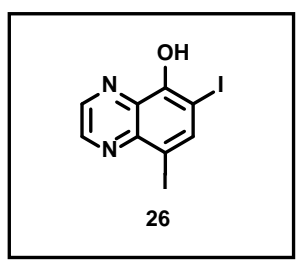

MP: $203-205^{\circ} \mathrm{C}$

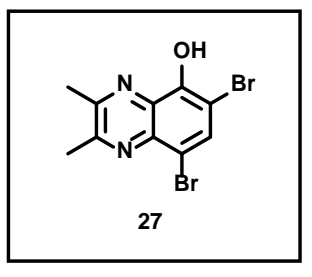

Yield: 67\% yield; 133 mg of $\mathbf{2 6}$ was isolated as a white solid.

${ }^{1}$ H NMR (400 MHz, d6-DMSO): $\delta 11.60$ (s, 1H), 9.03 (d, $J=1.8$ Hz, 1H), 8.90 (d, $J=1.8$ $\mathrm{Hz}, 1 \mathrm{H}), 8.60$ (s, $1 \mathrm{H})$.

${ }^{13}$ C NMR (100 MHz, $\boldsymbol{d}_{6}$-DMSO): $\delta$ 155.1, 147.2, 147.0, 144.3, 142.1, 133.0, 89.1, 82.7.

HRMS (DART): calc. for $\mathrm{C}_{8} \mathrm{H}_{5} \mathrm{I}_{2} \mathrm{~N}_{2} \mathrm{O}[\mathrm{M}+\mathrm{H}]^{+}$: 398.8486, found: 398.8500 .

Yield: 95\% yield; $142 \mathrm{mg}$ of 27 was isolated as an off-white solid.

${ }^{1}$ H NMR (400 MHz, CDCl M): $\delta 8.07$ (br. s, 1H), 7.97 (s, 1H), 2.75 (s, 3H), 2.72 (s, 3H).

${ }^{13}$ C NMR (100 MHz, $\left.\mathbf{C D C l}_{3}\right): \delta$ 155.8, 153.1, 148.8, 138.0, 135.4, 131.3, 112.0, 103.7, 23.5, 22.9 .

Note: NMR spectra match those previously reported. ${ }^{1}$ 


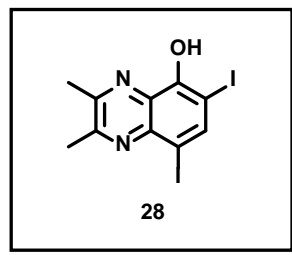

MP: $145-147^{\circ} \mathrm{C}$.

Yield: $72 \%$ yield; $84.9 \mathrm{mg}$ of $\mathbf{2 8}$ was isolated as an off-white solid.

${ }^{1}$ H NMR (400 MHz, $\left.\mathbf{C D C l}_{3}\right): \delta 8.39$ (s, 1H), 8.31 (br. s, 1H), 2.76 (s, 3H), 2.72 (s, 3H).

${ }^{13} \mathrm{C}$ NMR (100 MHz, CDCl 3 ): $\delta$ 156.3, 153.1, 153.0, 146.2, 140.7, 130.1, 88.5, 77.0, 23.4, 22.6.

HRMS (DART): calc. for $\mathrm{C}_{10} \mathrm{H}_{9} \mathrm{I}_{2} \mathrm{~N}_{2} \mathrm{O}[\mathrm{M}+\mathrm{H}]^{+}$: 426.8799, found: 426.8813.<smiles>Oc1cccc2nc3ccccc3nc12</smiles>

42

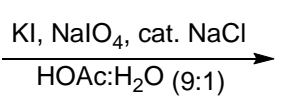
Synthesis of 2,4-diiodo-1-hydroxyphenazine (4): To a mixture of 1-hydroxyphenazine 42 (68.1 mg, 0.35
$\mathrm{mmol}$ ) in $15 \mathrm{~mL}$ of glacial acetic acid:water (9:1) was added sodium chloride (244 mg, $4.17 \mathrm{mmol})$, sodium periodate (446 mg, $2.08 \mathrm{mmol}$ ), then potassium iodide (241 mg, $2.08 \mathrm{mmol}$ ). The reaction was then allowed to stir at room temperature for 8 hours. Upon completion of this reaction (as determined by TLC analysis using dichloromethane), the reaction contents were transferred to a separatory funnel containing a solution of saturated sodium bicarbonate, and the aqueous layer was extracted with dichloromethane. The organic layer was collected, dried with anhydrous sodium sulfate and concentrated in vacuo. The resulting solid was adsorbed onto silica gel (dry loading with dichloromethane) and purified via column chromatography using first hexanes to elute an undesired purple product, followed by dichloromethane to elute pure 4 (19\%, $79.2 \mathrm{mg})$, which was isolated as a yellow solid. Note: This is a modified protocol from a previously published procedure. ${ }^{3}$

${ }^{1}$ H NMR (400 MHz, CDCl $)$ ): $\delta 8.78$ (br. s, 1H), 8.65 (s, 1H), 8.40 (m, 1H), 8.27 (m, 1H), 7.97 - 7.88 (m, $\left.2 \mathrm{H}\right)$.

${ }^{13} \mathrm{C}$ NMR (100 MHz, $\left.\mathbf{C D C l}_{3}\right): \delta 153.9,148.1,144.9,142.3,141.7,133.1,132.1,131.8,130.3,128.9,89.6$, 76.5 .

HRMS (DART): calc. for $\mathrm{C}_{12} \mathrm{H}_{7} \mathrm{I}_{2} \mathrm{~N}_{2} \mathrm{O}[\mathrm{M}+\mathrm{H}]^{+}:$448.8642, found: 448.8623.

MP: 193 - $195^{\circ} \mathrm{C}$.
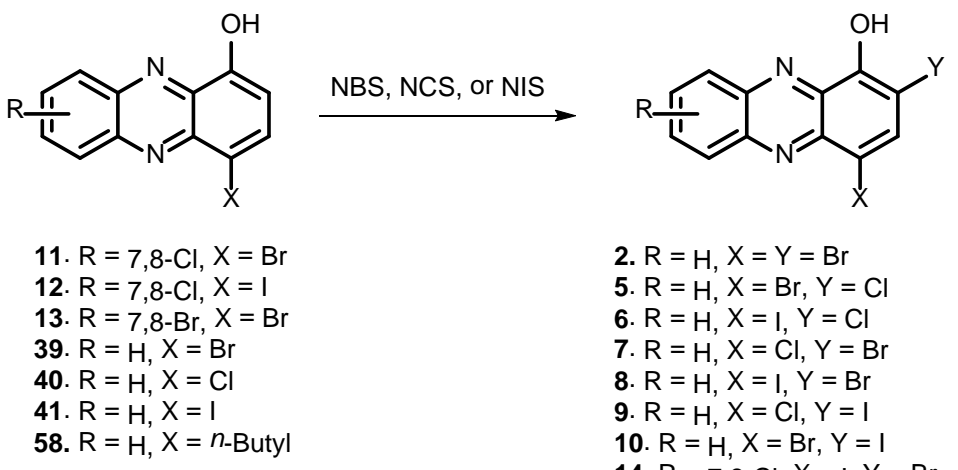

$$
\begin{aligned}
& \text { 2. } R=H, X=Y=B r \\
& \text { 5. } R=H, X=B r, Y=C l \\
& \text { 6. } R=H, X=I, Y=C l \\
& \text { 7. } R=H, X=C l, Y=B r \\
& \text { 8. } R=H, X=I, Y=B r \\
& \text { 9. } R=H, X=C l, Y=I \\
& \text { 10. } R=H, X=B r, Y=I \\
& \text { 14. } R=7,8-C l, X=I, Y=B r \\
& \text { 15. } R=7,8-B r, X=B r, Y=C l \\
& \text { 24. } R=H, X=n-B u t y l, Y=B r
\end{aligned}
$$


General monohalogenation procedure for synthesis of 2, 5-10, 14-15 and 22: 4-Chloro-1-hydroxyphenazine 40 (75.4 mg, $0.33 \mathrm{mmol}$ ) and $N$-bromosuccinimide (58.2 mg, $0.33 \mathrm{mmol}$ ) were dissolved in $15 \mathrm{~mL}$ dichloromethane and allowed to stir at room temperature for 1 hour. The reaction contents were then concentrated, adsorbed onto silica gel and purified via column chromatography using dichloromethane to elute 2-bromo-4-chloro-1-hydroxyphenazine 7, which was isolated as a yellow solid (91\%, $91.9 \mathrm{mg})$. Note: Analogous procedures were used for chlorination and iodination using $N$-chlorosuccinimide and $N$ iodosuccinimide respectively.

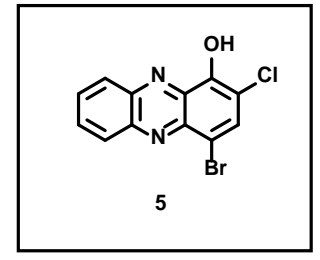

Yield: 34\% yield; $39.4 \mathrm{mg}$ of 5 was isolated as a yellow solid.

${ }^{1}$ H NMR (400 MHz, CDCl $)$ ): $\delta 8.44-8.35$ (m, 2H), $8.26(\mathrm{~m}, 1 \mathrm{H}), 8.13(\mathrm{~s}, 1 \mathrm{H}), 7.96-$ $7.89(\mathrm{~m}, 2 \mathrm{H})$.

${ }^{13}$ C NMR (100 MHz, $\left.\mathbf{C D C l}_{3}\right): \delta$ 147.6, 144.2, 141.6, 139.7, 135.4, 134.6, 132.3, 131.8, 130.4, 128.9, 115.2, 112.9 .

HRMS (DART): calc. for $\mathrm{C}_{12} \mathrm{H}_{7} \mathrm{BrClN}_{2} \mathrm{O}[\mathrm{M}+\mathrm{H}]^{+}$: 308.9425, found: 308.9437.

MP: $229-231^{\circ} \mathrm{C}$.

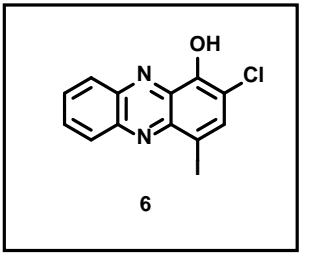

Yield: $42 \%$ yield; $17.3 \mathrm{mg}$ of $\mathbf{6}$ was isolated as a yellow solid.

${ }^{1}$ H NMR (400 MHz, $\left.\mathbf{C D C l}_{3}\right): \delta 8.43$ (br. s, $\left.1 \mathrm{H}\right), 8.39$ (m, 1H), 8.38 (s, 1H), 8.26 (m, 1H), $7.96-7.89(\mathrm{~m}, 2 \mathrm{H})$.

${ }^{13} \mathrm{C}$ NMR (100 MHz, CDCl 3$): \delta 148.5,144.6,141.9,141.7,141.3,134.0,132.2,131.7$, 130.3, 128.8, 116.0, 88.9.

HRMS (DART): calc. for $\mathrm{C}_{12} \mathrm{H}_{7} \mathrm{ClIN} \mathrm{N}_{2} \mathrm{O}[\mathrm{M}+\mathrm{H}]^{+}$: 356.9286, found: 356.9281 .

MP: $183-185^{\circ} \mathrm{C}$.

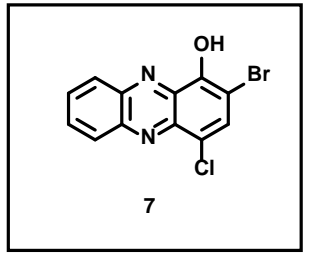

Yield: 91\% yield; $91.9 \mathrm{mg}$ of 7 was isolated as a yellow solid.

${ }^{1} H_{\text {NMR (400 MHz, CDCl }}$ ): $\delta 8.47$ (br. s, 1H), 8.39 (m, 1H), 8.25 (m, 1H), 8.03 (s, 1H), $7.99-7.86(\mathrm{~m}, 2 \mathrm{H})$.

${ }^{13}$ C NMR (100 MHz, $\left.\mathbf{C D C l}_{3}\right): \delta$ 148.6, 144.0, 141.6, 139.4, 134.4, 134.0, 132.3, 131.9, 130.5, 129.1, 123.4, 102.6.

HRMS (DART): calc. for $\mathrm{C}_{12} \mathrm{H}_{7} \mathrm{BrClN}_{2} \mathrm{O}[\mathrm{M}+\mathrm{H}]^{+}$: 308.9425 , found: 308.9434 .

MP: $212-214^{\circ} \mathrm{C}$.

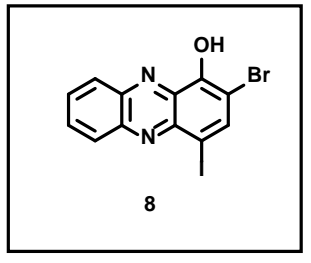

Yield: 65\% yield; $88.1 \mathrm{mg}$ of $\mathbf{8}$ was isolated as a yellow solid.

${ }^{1}$ H NMR (400 MHz, CDCl 3 ): $\delta 8.57$ (br. s, 1H), 8.51 (s, 1H), 8.40 (m, 1H), 8.27 (m, 1H), $7.97-7.89(\mathrm{~m}, 2 \mathrm{H})$.

${ }^{13}$ C NMR (100 MHz, $\left.\mathbf{C D C l}_{3}\right): \delta$ 150.2, 144.7, 143.8, 141.7, 141.7, 134.0, 132.2, 131.8, 130.3, 128.8, 104.2, 89.2.

HRMS (DART): calc. for $\mathrm{C}_{12} \mathrm{H}_{7} \mathrm{BrIN}_{2} \mathrm{O}[\mathrm{M}+\mathrm{H}]^{+}: 400.8781$, found: 400.8785 .

MP: $186-188^{\circ} \mathrm{C}$. 


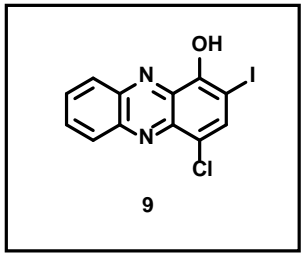

Yield: 82\% yield; $75.4 \mathrm{mg}$ of $\mathbf{9}$ was isolated as a yellow solid.

${ }^{1}$ H NMR (400 MHz, CDCl $): \delta 8.67$ (br. s, 1H), 8.36 (m, 1H), 8.21 (m, 1H), 8.14 (s, 1H), $7.98-7.85$ (m, 2H).

${ }^{13}$ C NMR (100 MHz, CDCl $): \delta$ 152.2, 144.1, 141.4, 139.8, 138.4, 133.3, 132.2, 131.9, 130.4, 129.1, 123.4, 74.4.

HRMS (DART): calc. for $\mathrm{C}_{12} \mathrm{H}_{7} \mathrm{ClIN}_{2} \mathrm{O}[\mathrm{M}+\mathrm{H}]^{+}$: 356.9286, found: 356.9299 .

MP: $175-177^{\circ} \mathrm{C}$.

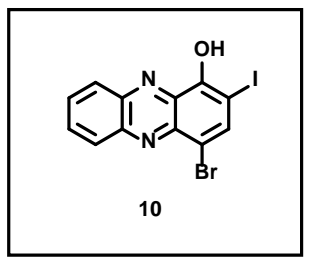

Yield: 48\% yield; $20.7 \mathrm{mg}$ of $\mathbf{1 0}$ was isolated as a yellow solid.

${ }^{1}$ H NMR (400 MHz, CDCl3): $\delta 8.71$ (br. s, 1H), 8.36 (m, 1H), 8.35 (s, 1H), 8.22 (m, 1H), $7.95-7.87$ (m, 2H).

${ }^{13}$ C NMR (100 MHz, $\left.\mathbf{C D C l}_{3}\right): \delta$ 152.8, 144.4, 141.7, 141.4, 140.6, 133.4, 132.2, 131.9, 130.3, 129.0, 113.3, 75.2.

HRMS (DART): calc. for $\mathrm{C}_{12} \mathrm{H}_{7} \mathrm{BrIN}_{2} \mathrm{O}[\mathrm{M}+\mathrm{H}]^{+}:$: 400.8781, found: 400.8770 .

MP: $188-190{ }^{\circ} \mathrm{C}$.

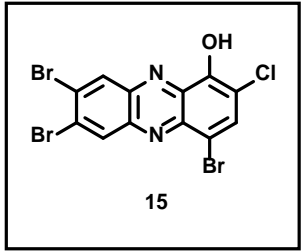

MP: $242-244{ }^{\circ} \mathrm{C}$

Yield: $65 \%$ yield; $15.8 \mathrm{mg}$ of $\mathbf{1 5}$ was isolated as an orange solid.

${ }^{1}$ H NMR (400 MHz, $\boldsymbol{d}_{6}$-DMSO): $\delta 8.78$ (s, 1H), 8.68 (s, 1H), 8.41 (s, 1H).

${ }^{13}$ C NMR (100 MHz, $\boldsymbol{d}_{6}$-DMSO): $\delta$ 149.5, 141.7, 140.4, 139.7, 136.3, 135.9, 133.3, 132.6, 128.2, 128.1, 116.5, 111.3.

HRMS (DART): calc. for $\mathrm{C}_{12} \mathrm{H}_{5} \mathrm{Br}_{3} \mathrm{ClN}_{2} \mathrm{O}[\mathrm{M}+\mathrm{H}]^{+}$: 464.7635 , found: 464.7614 .

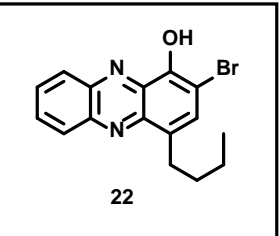

Yield: 53\% yield; $12.9 \mathrm{mg}$ of $\mathbf{2 2}$ was isolated as a yellow solid.

${ }^{1}$ H NMR (400 MHz, $\left.\mathbf{C D C l}_{3}\right): \delta 8.38$ (br. s, 1H), 8.28 (m, 1H), 8.21 (m, 1H), 7.90 - 7.80 (m, 2H), 7.67 (t, $J=0.8 \mathrm{~Hz} 1 \mathrm{H}), 3.26$ (dt, $J=7.8,0.9 \mathrm{~Hz}, 2 \mathrm{H}), 1.83-1.75$ (m, 2H), 1.47 (tq, $J=$ 7.4, $7.4 \mathrm{~Hz}, 2 \mathrm{H}), 0.99$ (t, $J=7.4 \mathrm{~Hz}, 3 \mathrm{H})$.

${ }^{13}$ C NMR (100 MHz, $\left.\mathbf{C D C l}_{3}\right): \delta$ 147.2, 143.4, 142.0, 141.0, 134.7, 134.0, 132.9, 131.2, 130.6, 130.5, 129.0, 103.5, 32.9, 30.1, 22.9, 14.3.

HRMS (ESI): calc. for $\mathrm{C}_{16} \mathrm{H}_{16} \mathrm{BrN}_{2} \mathrm{O}[\mathrm{M}+\mathrm{H}]^{+}$: 331.0441, found: 331.0462 .

MP: $135-137^{\circ} \mathrm{C}$.
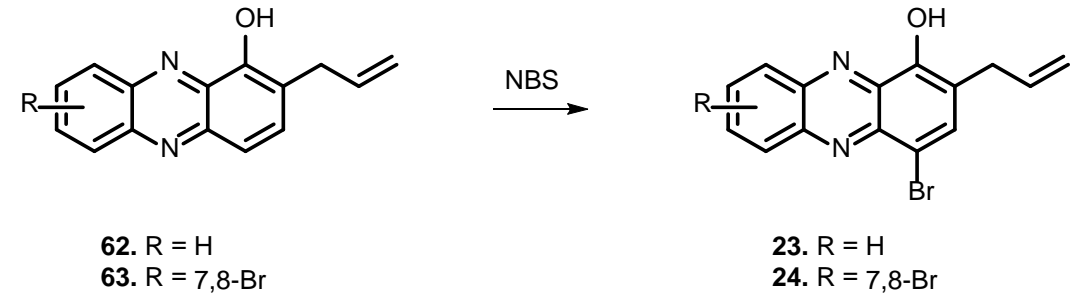

23. $\mathrm{R}=\mathrm{H}$

24. $\mathrm{R}=7,8-\mathrm{Br}$

General halogenation procedure for synthesis of 23 and 24: 2-Allyl-1-hydroxyphenazine 62 (46.5 mg, 0.20 mmol) and $N$-bromosuccinimide (35.1 mg, $0.20 \mathrm{mmol}$ ) were dissolved in $10 \mathrm{~mL}$ dichloromethane and allowed to stir at room temperature for 1 hour. The reaction contents were then concentrated, adsorbed onto silica gel 
and purified via column chromatography using dichloromethane to elute 2-allyl-4-bromo-1-hydroxyphenazine 23, which was isolated as a yellow solid (93\%, $58.8 \mathrm{mg})$. Note: An analogous procedure was used to synthesize 24.

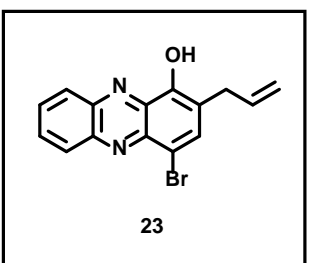

Yield: 93\% yield; $58.8 \mathrm{mg}$ of $\mathbf{2 3}$ was isolated as a yellow solid.

${ }^{1} \mathbf{H}$ NMR (400 MHz, CDCl $): \delta 8.35(\mathrm{~m}, 1 \mathrm{H}), 8.22(\mathrm{~s}, 1 \mathrm{H}), 8.16(\mathrm{~m}, 1 \mathrm{H}), 7.98(\mathrm{~s}, 1 \mathrm{H})$, $7.92-7.76$ (m, 2H), 6.06 (ddt, $J=16.7,10.1,6.6 \mathrm{~Hz}, 1 \mathrm{H}), 5.21$ (ddt, $J=16.7,1.7,1.6 \mathrm{~Hz}$, 1H), 5.17 (ddt, $J=10.1,1.7,1.5 \mathrm{~Hz}, 1 \mathrm{H}) 3.66$ (ddd, $J=6.6,1.6,1.5 \mathrm{~Hz}, 2 \mathrm{H}$ ).

${ }^{13}$ C NMR (100 MHz, CDCl $): \delta$ 148.2, 144.0, 141.4, 140.0, 136.9, 135.4, 134.8, 131.3, 131.0, 130.3, 128.9, 121.8, 117.1, 112.0, 33.8.

HRMS (ESI): calc. for $\mathrm{C}_{15} \mathrm{H}_{12} \mathrm{BrN}_{2} \mathrm{O}[\mathrm{M}+\mathrm{H}]^{+}:$315.0128, found: 315.0127 .

MP: $149-151^{\circ} \mathrm{C}$.

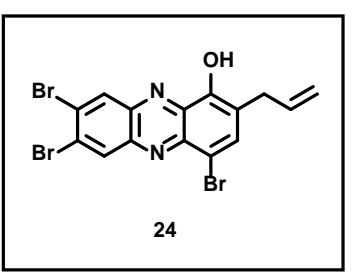

Yield: $39 \%$ yield; $17.7 \mathrm{mg}$ of $\mathbf{2 4}$ was isolated as a yellow solid.

${ }^{1}$ H NMR (400 MHz, CDCl 3 ): $\delta 8.74$ (s, 1H), 8.57 (s, 1H), 8.07 (br. s, 1H), 8.03 (s, 1H), 6.04 (ddt, $J=16.8,10.1,6.6 \mathrm{~Hz}, 1 \mathrm{H}$ ), 5.21 (dd, 16.8, $1.6 \mathrm{~Hz}, 1 \mathrm{H}), 5.19$ (dd, 10.1, $1.6 \mathrm{~Hz}$, $1 \mathrm{H}), 3.66$ (d, $J=6.6 \mathrm{~Hz}, 2 \mathrm{H})$.

${ }^{13}$ C NMR (100 MHz, CDCl $): \delta 148.2,142.7,140.4,140.2,138.1,135.3,135.0,134.0$, 132.6, 128.9, 128.5, 123.1, 117.5, 112.2, 33.8.

HRMS (ESI): calc. for $\mathrm{C}_{15} \mathrm{H}_{14} \mathrm{Br}_{3} \mathrm{~N}_{2} \mathrm{O}[\mathrm{M}+\mathrm{H}]^{+}$: 472.8318, found: 472.8331 .

MP: $187-189^{\circ} \mathrm{C}$. 


\section{3.) Biological Methods:}

\section{A.) Minimum Inhibitory Concentration (MIC) Susceptibility Assay (in 96-well plate):}

The minimum inhibitory concentration (MIC) for each phenazine analogue was determined by the broth microdilution method as recommended by the Clinical and Laboratory Standards Institute (CLSI). ${ }^{4}$ In a 96-well plate, eleven two-fold serial dilutions of each compound were made in a final volume of $100 \mu \mathrm{L}$ Luria Broth. Each well was inoculated with $\sim 10^{5}$ bacterial cells at the initial time of incubation, prepared from a fresh log phase culture (OD 600 of 0.5 to 1.0 depending on bacterial strain). The MIC was defined as the lowest concentration of compound that prevented bacterial growth after incubating 16 to 18 hours at $37{ }^{\circ} \mathrm{C}$ (MIC values were supported by spectrophotometric readings at $\mathrm{OD}_{600}$ ). The concentration range tested for each phenazine analogue/antibacterial during this study was 0.10 to $100 \mu \mathrm{M}$. DMSO served as our vehicle and negative control in each microdilution MIC assay. DMSO was serially diluted with a top concentration of $1 \%$ v/v.

Supporting Table 1: MIC values for select compounds tested against a panel of gram-negative bacteria.

\begin{tabular}{|c|c|c|c|c|}
\hline Compound & $\begin{array}{c}\text { A. baumannii } \\
19606 \\
\text { MIC }(\mu \mathrm{M})\end{array}$ & $\begin{array}{c}\text { PAO1 } \\
\text { MIC }(\mu \mathrm{M})\end{array}$ & $\begin{array}{c}\text { K. pneumoniae } 13883 \\
\text { MIC }(\mu \mathrm{M})\end{array}$ & $\begin{array}{l}\text { E. coli UAEC-1 } \\
\text { MIC }(\mu \mathrm{M})\end{array}$ \\
\hline 2 & 100 & $>100$ & $>100$ & $>100$ \\
\hline 8 & 50 & $>100$ & $>100$ & $>100$ \\
\hline 11 & $>100$ & $>100$ & $>100$ & $>100$ \\
\hline 12 & $>100$ & $>100$ & $>100$ & $>100$ \\
\hline 13 & $>100$ & $>100$ & $>100$ & $>100$ \\
\hline 14 & $>100$ & -- & -- & -- \\
\hline 20 & $>100$ & $>100$ & $>100$ & $>100$ \\
\hline 21 & $>100$ & $>100$ & $>100$ & $>100$ \\
\hline 19 & $>100$ & $>100$ & $>100$ & $>100$ \\
\hline 25 & 6.25 & $>100$ & $37.5^{\mathrm{a}}$ & 12.5 \\
\hline 27 & $>100$ & $>100$ & $>100$ & $>100$ \\
\hline 28 & $>100$ & $>100$ & $>100$ & $>100$ \\
\hline Ciprofloxacin & 6.25 & 0.78 & 0.78 & $0.15^{\mathrm{a}}$ \\
\hline
\end{tabular}

Note: ${ }^{\mathrm{a}}$ represents the mid-point of a two-fold range of values. 
Supporting Table 2: MIC values after addition of $1 \mathrm{mM}$ Tiron and $200 \mu \mathrm{M} \mathrm{CuSO}_{4}$ along with the fold change.

S. epidermidis 12228

\begin{tabular}{c|c|cc|cc} 
Compound & \multicolumn{1}{c}{ MIC } & MIC w/ Tiron & Fold $\Delta$ & MIC w/ $\mathrm{Cu}^{2+}$ & Fold $\Delta$ \\
\hline 2 & 1.56 & $1.17^{\mathrm{a}}$ & n.a. & 50 & +32 \\
8 & 1.56 & $1.17^{\mathrm{a}}$ & n.a. & 12.5 & +8 \\
12 & 0.39 & $0.30^{\mathrm{a}}$ & n.a. & 0.39 & n.a. \\
13 & $0.1^{\mathrm{b}}$ & $0.1^{\mathrm{b}}$ & n.a. & 50 & +500 \\
15 & $0.1^{\mathrm{b}}$ & $0.1^{\mathrm{b}}$ & n.a. & 50 & +500 \\
28 & 1.56 & $1.17^{\mathrm{a}}$ & n.a. & 0.39 & +4 \\
$8-\mathrm{HQ}$ & 3.13 & $>100$ & $>+32$ & 3.13 & n.a. \\
\hline \multicolumn{5}{c}{} \\
Compound & MIC & MIC w/ Tiron & Fold $\Delta$ & MIC w/ $\mathrm{Cu}^{2+}$ & Fold $\Delta$ \\
\hline 2 & 6.25 & 6.25 & n.a. & 12.5 & n.a. \\
8 & 3.13 & 3.13 & n.a. & 3.13 & n.a. \\
12 & 3.13 & 3.13 & n.a. & 1.56 & n.a. \\
13 & 0.39 & 0.39 & n.a. & 25 & +64 \\
15 & 0.78 & 0.78 & n.a. & 12.5 & +16 \\
28 & 6.25 & 6.25 & n.a. & $2.35^{\mathrm{a}}$ & n.a. \\
$8-H Q$ & $18.8^{\mathrm{a}}$ & $>100$ & $>+12$ & $18.8^{\mathrm{a}}$ & n.a.
\end{tabular}

Note: "Fold $\Delta$ " denotes the change in antibacterial activity according to MIC values; $(+)$ for increase in MIC value/loss of antibacterial activity; (-) for decrease in MIC value/increase of antibacterial activity; (n.a.) means insignificant changes in MIC values ( $\leq 2$-fold changes). ${ }^{a}$ represents the mid-point of a two-fold range of values.

\section{B.) MIC Assay with Tiron or Metal (II) Cation Co-Treatment:}

Halogenated phenazines were co-treated with tiron in MIC assays to determine if select halogenated phenazine compounds demonstrated antibacterial activities as a result of redox-activity. We did not observe tiron suppression of antibacterial activities of these HP analogues, but we did observe a loss in antibacterial activity of 8-hydroxyquinoline (a redox-active control). Results from these experiments are summarized in Supporting Tables 1 and 2. Metal (II) cation studies were performed in a similar setup to the standard MIC assay, with the addition of $200 \mu \mathrm{M}$ of the metal salt (Copper (II) sulfate, Magnesium (II) sulfate and Ammonium iron (II) sulfate hexahydrate) to the LB medium in the MIC assays. ${ }^{5}$

C.) HP 2 and 29 Complex Formation with $\mathbf{C u}($ II) and Fe(II). The rates of phenazine-metal(II) and quinolinemetal(II) complex formation were independently evaluated via UV-vis spectrometry following addition of 0.5 equivalents $\mathrm{CuSO}_{4}$ or Ammonium iron(II) sulfate hexahydrate to stirring solutions of HP 2 or $29(10 \mathrm{mM}, 20$ $\mathrm{mL}$ ) in dimethyl sulfoxide. Spectral scanning was performed from 200 to $900 \mathrm{~nm}$ in $2 \mathrm{~nm}$ increments. The $\lambda_{\max }$ values were determined to be $440 \mathrm{~nm}$ and $314 \mathrm{~nm}$ for 2 and 29 respectively. The disappearance of each complex was observed over the indicated time points. Although the copper(II) complex of $\mathbf{2 9}$ yielded an easily discernable UV-vis spectrum $\left(\lambda_{\max }=394 \mathrm{~nm}\right)$, the phenazine-copper complex formation yielded a loss in absorbance due to precipitation. Due to the insolubility of the phenazine:copper complex, the stoichiometry was determined by spectrophotometrically quantifying the concentration of free phenazine in solution following incubation with varying equivalents of $\mathrm{CuSO}_{4}$ (Supporting Figure 1; Supporting Information). Each data point was evaluated in independent experiments wherein $10 \mathrm{mM}$ solutions of HP 2 in methanol $(15 \mathrm{~mL})$ were stirred with $\mathrm{CuSO}_{4}$ for 2 hours at room temperature. After this time, the mixtures were filtered and the solvent was removed in vacuo to afford free HP 2 as a solid. HP 2 solid was then dissolved in $15 \mathrm{~mL}$ of dimethyl sulfoxide and the absorbance at $440 \mathrm{~nm}$ was evaluated after a single ten-fold dilution was made. The concentrations of 
free HP 2 in solution could thus be determined from a calibration curve of serial dilutions of HP 2 in dimethyl sulfoxide (Supporting Figure 2). This data demonstrates $>99 \%$ of HP 2 (ligand) is bound to copper(II) at a minimum of 2:1 ligand:copper ratio, suggesting the stoichiometry of the ligand:metal complex is 2:1.
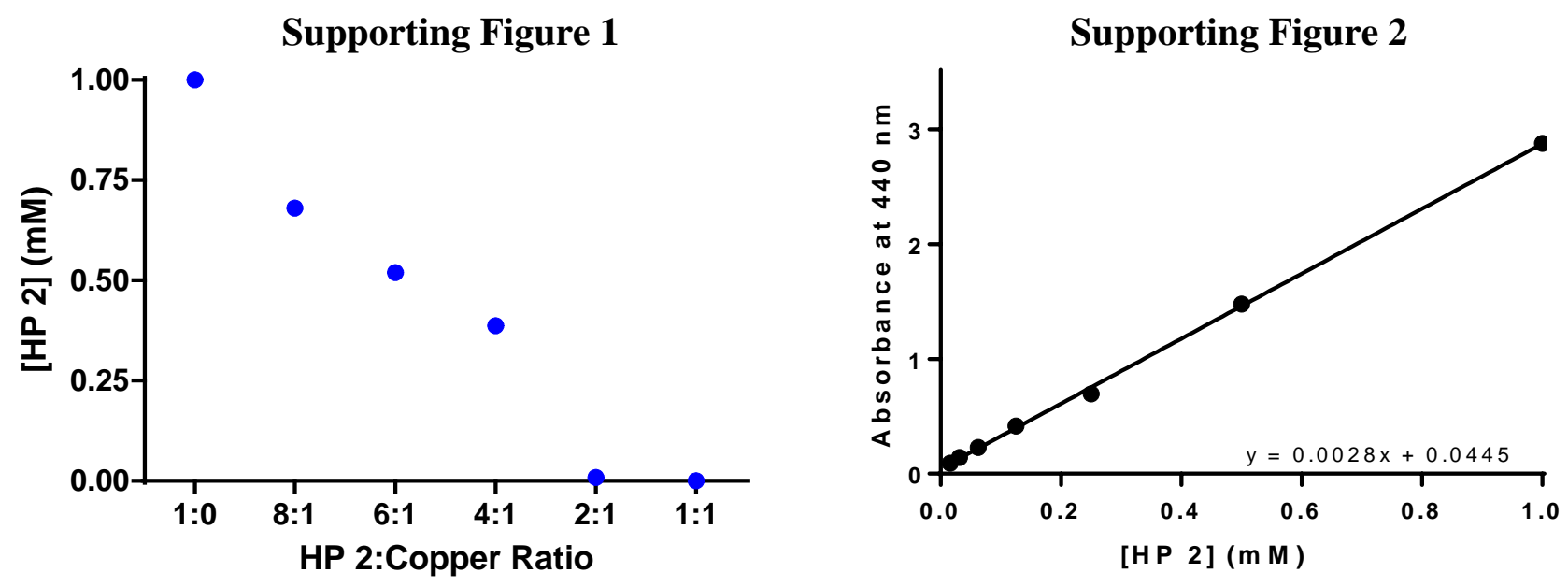

\section{D.) MIC Assay for Mycobacterium tuberculosis:}

M. tuberculosis H37Ra (ATCC 25177) was inoculated in $10 \mathrm{~mL}$ Middlebrook 7H9 medium and allowed to grow for two weeks. The culture was then diluted with fresh medium until an $\mathrm{OD}_{600}$ of 0.01 was reached. Aliquots of $200 \mu \mathrm{L}$ were then added to each well of a 96-well plate starting from the second column. Test compounds were dissolved in DMSO at final concentration of $10 \mathrm{mM}$. $7.5 \mu \mathrm{L}$ of each compound along with DMSO (negative control) and streptomycin (positive control-40mg/ml stock solution) were added to $1.5 \mathrm{~mL}$ of the Mycobacterium diluted cultures, resulting in $50 \mu \mathrm{M}$ final concentration of each halogenated phenazine analogues and $340 \mu \mathrm{M}$ for streptomycin. The final DMSO concentration was maintained at $0.5 \%$. Aliquots of $400 \mu \mathrm{l}$ were added to wells of the first column of the 96-well plate and serially diluted two-fold (200 $\mu \mathrm{l})$ per well across the plate to obtain final concentrations that ranges from 0.024 to $50 \mu \mathrm{M}$ for the test compounds and 0.16 to $340 \mu \mathrm{M}$ for streptomycin. Three rows were reserved for each compound. The plates were then incubated at $37^{\circ} \mathrm{C}$ for seven days. Minimum inhibitory concentrations are reported as the lowest concentration at which no bacterial growth was observed. OD 600 absorbance was recorded using SpectraMax M5 (Molecular Devices). Data obtained from three independent experiments were analyzed using Excel.

\section{E.) Calgary Biofilm Device (CBD) Experiments}

Minimum Bactericidal Concentrations (MBC) and Minimum Biofilm Eradication Concentrations (MBEC) Determination

Biofilm eradication experiments were performed using the Calgary Biofilm Device to determine MBC/MBEC values for various compounds of interest (Innovotech, product code: 19111). ${ }^{6}$ The Calgary device (96-well plate with lid containing pegs to establish biofilms on) was inoculated with $125 \mu \mathrm{L}$ of a mid-log phase culture diluted 1,000 -fold in tryptic soy broth with $0.5 \%$ glucose (TSBG) to establish bacterial biofilms after incubation at 37 ${ }^{\circ} \mathrm{C}$ for 24 hours. The lid of the Calgary device was then removed, washed and transferred to another 96-well plate containing 2-fold serial dilutions of the test compounds (the "challenge plate”). The total volume of media with compound in each well in the challenge plate is $150 \mu \mathrm{L}$. The Calgary device was then incubated at $37^{\circ} \mathrm{C}$ for 24 hours. The lid was then removed from the challenge plate and MBC/MBEC values were determined using different final assays. To determine MBC values, $20 \mu \mathrm{L}$ of the challenge plate was transferred into a fresh 96-well plate containing $180 \mu \mathrm{L}$ TSBG and incubated overnight at $37{ }^{\circ} \mathrm{C}$. The MBC values were S19 
determined as the concentration giving a lack of visible bacterial growth (i.e., turbidity). For determination of MBEC values, the Calgary device lid (with attached pegs/treated biofilms) was transferred to a new 96-well plate containing $150 \mu \mathrm{L}$ of fresh TSBG media in each well and incubated for 24 hours at $37{ }^{\circ} \mathrm{C}$ to allow viable biofilms to grow and disperse resulting in turbidity after the incubation period. MBEC values were determined as the lowest test concentration that resulted in eradicated biofilm (i.e., wells that had no turbidity after final incubation period). In select experiments, pegs from the Calgary device were removed from lead biofilm eradicators after final incubation, sonicated for 30 minutes in PBS and plated out to determine biofilm cell killing of lead biofilm-eradicating agents (i.e., colony forming unit per milliliter, CFU/mL; see below).

Note: MRSA-2 (clinical isolate from Shands Hospital, Gainesville, FL), S. aureus (MRSA ATCC strains: BAA-1707, BAA-44), S. epidermidis (MRSE; ATCC 35984) and E. faecium (VRE; ATCC 700221) were tested using these assay parameters.
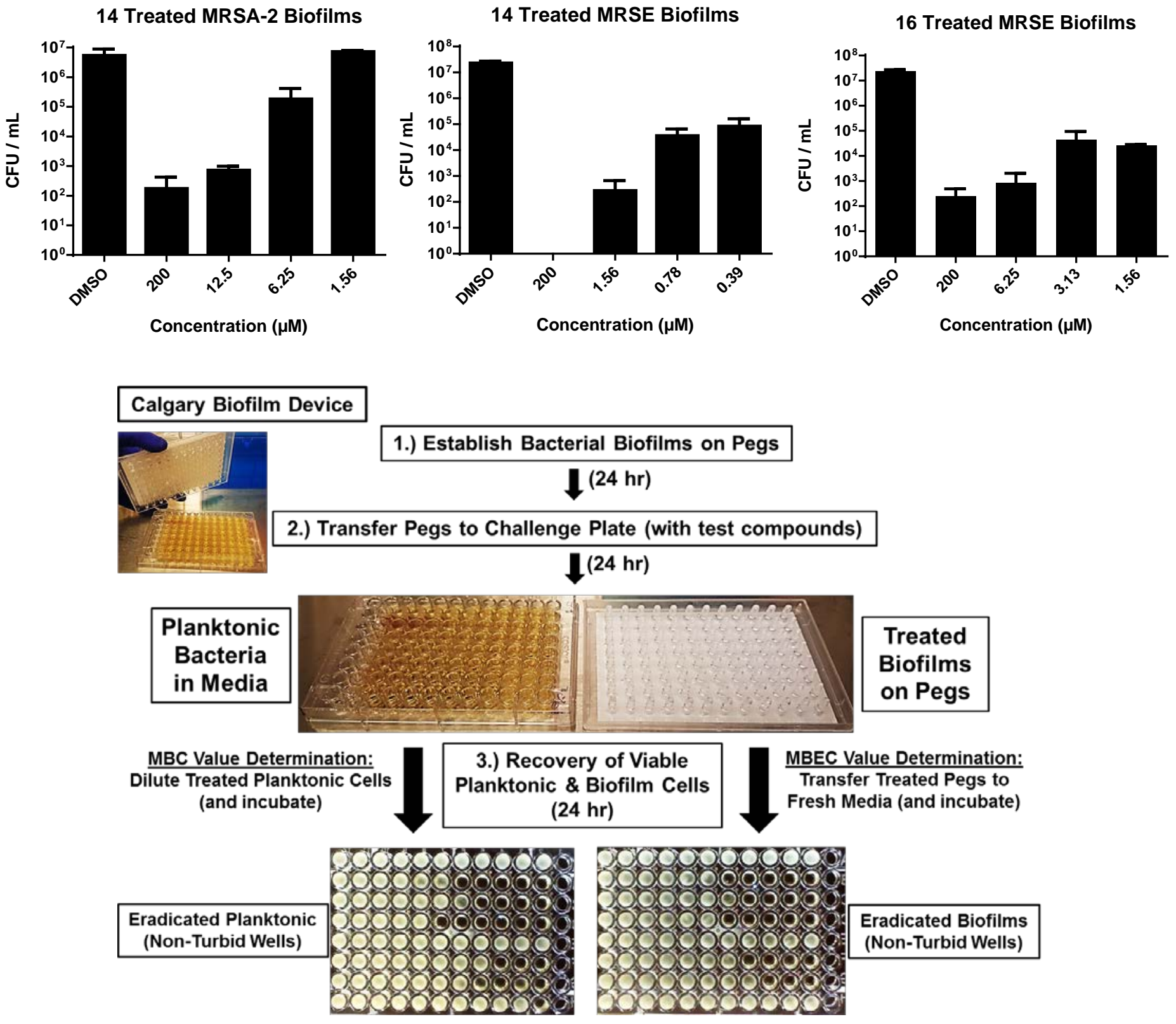

Work flow for the determination of MBC and MBEC values using the Calgary Biofilm Device. 


\section{F.) Kill Kinetics of Exponential Growth Cultures (Rapidly-Dividing Bacteria):}

An overnight culture of MRSA-2, S. epidermidis (ATCC 12228) or E. faecium (VRE 700221) was diluted 1:1,000 in $10 \mathrm{~mL}$ of LB media (Brain heart infusion media was used for $E$. faecium) in test tubes containing test compounds. These test tubes were incubated at $37^{\circ} \mathrm{C}$ with shaking at $250 \mathrm{rpm}$. At $0,1,3,6,9$ and 24 hours, $100 \mu \mathrm{L}$ aliquots were removed from each test tubes, serial diluted and spread on LB $1 \%$ agar plates. The resulting plates were then incubated at $37^{\circ} \mathrm{C}$ overnight to allow viable colonies to grow. Following this incubation period, viable bacterial colonies were counted on each agar plate to determine colony forming units $(\mathrm{CFU}) / \mathrm{mL}$. Plates containing between 30 and 300 colonies were used for this analysis. Each experiment was conducted in three replicates from separate, individual bacterial colonies. Compounds were considered to be bactericidal at $\geq 3$ - $\log _{10}$ reduction in $\mathrm{CFU} / \mathrm{mL}$ compared to the vehicle (DMSO) control or bacteriostatic at $\leq 3$ $\log _{10} \mathrm{CFU} / \mathrm{mL}$ reduction compared to the vehicle (DMSO) control after 24 hours. Kill curves were plotted using Graphpad Prism 6.0. From these experiments, we concluded our HP analogues to operate through a bacteriostatic antibacterial mechanism as $\geq 3-\log _{10}$ reduction in $\mathrm{CFU} / \mathrm{mL}$ required concentrations $>4 \mathrm{X}$ MIC.

\section{Kill curves of rapidly-growing bacteria generated during these investigations:}

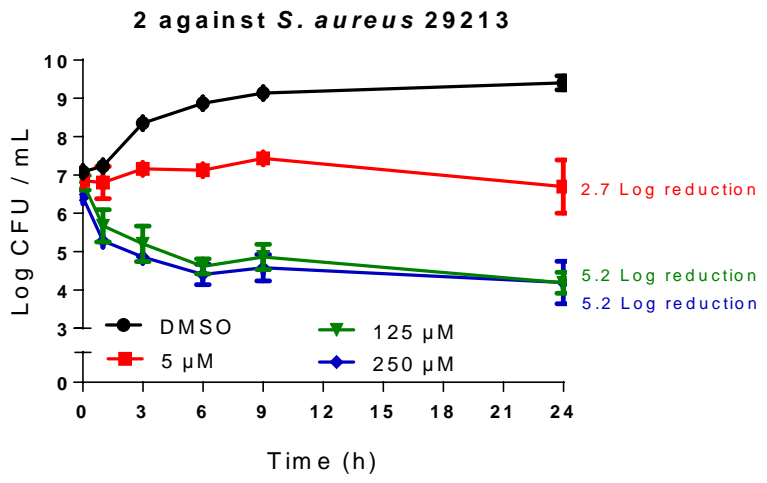

16 against $S$. epiderm id is 12228

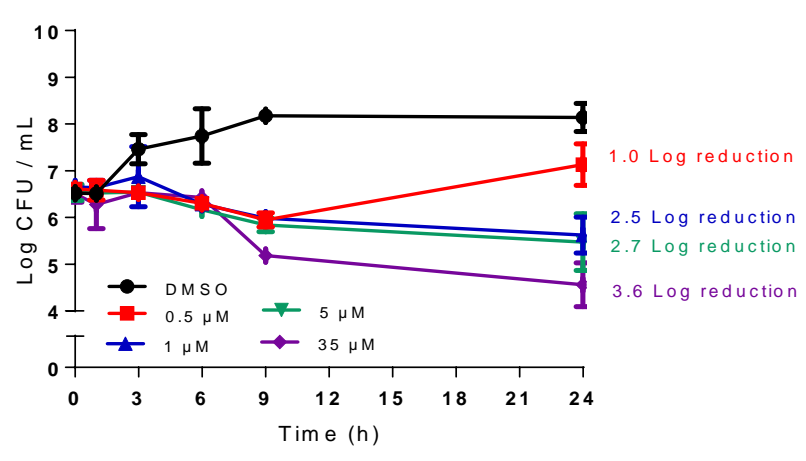

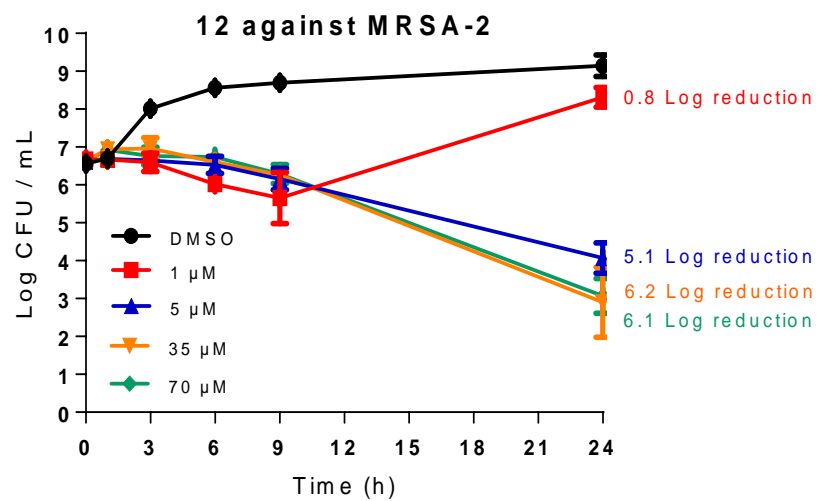

14 against E. faecium 700221

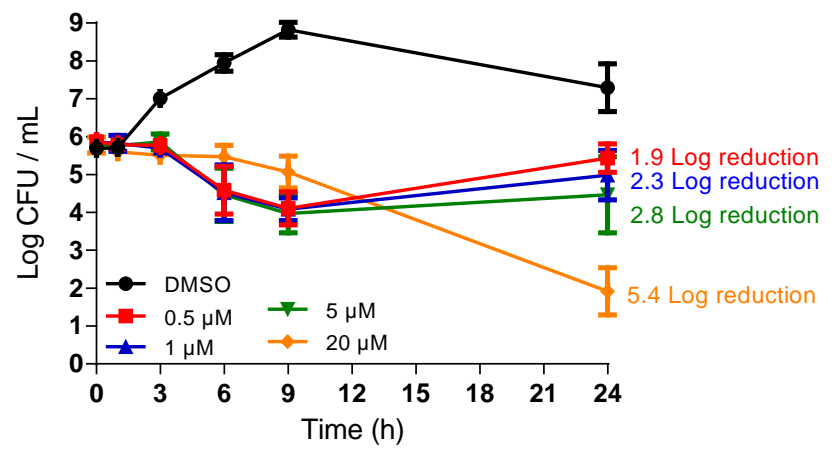




\section{G.) Live / Dead staining (Fluorescence Microscopy) of MRSE 35984 biofilms:}

A mid-log culture of MRSE 35894 was diluted 1:1,000-fold and $500 \mu \mathrm{L}$ was transferred to each compartment of a 4 compartment CELLview dish (Greiner Bio-One 627871). The dish was then incubated for 24 hours at 37 ${ }^{\circ} \mathrm{C}$. After this time, the cultures were removed and the plate was washed with $0.9 \%$ saline. The dish was then treated with the compounds in fresh media at various concentrations. DMSO was used as our negative control in this assay. The dish was incubated with the compound for 24 hours at $37^{\circ} \mathrm{C}$. After this time, the cultures were removed and the dish was washed with $0.9 \%$ saline for 2 minutes. Saline was then removed and $500 \mu \mathrm{L}$ of the stain (Live/Dead BacLight Viability Kit, Invitrogen) were added for 15 minutes and left in the dark. After this time, the stain was removed and the dish was washed twice with $0.9 \%$ saline. Then the dish was fixed with 500 $\mu \mathrm{L} 4 \%$ paraformaldehyde in PBS for 30 minutes. Images of remaining MRSE biofilms were then taken with a fluorescence microscope. All data were analyzed using Image J software.

\section{H.) Hemolysis Assay with Red Blood Cells:}

As previously described, freshly drawn human red blood cells (hRBC with ethylenediaminetetraacetic acid (EDTA) as an anticoagulant) were washed with Tris-buffered saline (0.01M Tris-base, $0.155 \mathrm{M}$ sodium chloride (NaCl), pH 7.2) and centrifuged for 5 minutes at 3,500 rpm. The washing was repeated three times with the buffer. In 96-well plate, test compounds were added to the buffer from DMSO stocks. Then 2\% hRBCs $(50 \mu \mathrm{L})$ in buffer were added to test compounds to give a final concentration of $200 \mu \mathrm{M}$. The plate was then incubated for 1 hour at $37^{\circ} \mathrm{C}$. After incubation, the plate was centrifuged for 5 minutes at 3,500 rpm. Then 80 $\mu \mathrm{L}$ of the supernatant was transferred to another 96-well plate and the optical density (OD) was read at $405 \mathrm{~nm}$. DMSO served as our negative control (0\% hemolysis) while Triton X served as our positive control (100\% hemolysis). The percent of hemolysis was calculated as (OD 405 of the compound- $\mathrm{OD}_{405} \mathrm{DMSO}_{\mathrm{S}} /\left(\mathrm{OD}_{405} \mathrm{Triton}\right.$ $\mathrm{X}-\mathrm{OD}_{405}$ buffer).

\section{I.) LDH Release Assay for HeLa Cytotoxicity Assessment:}

HeLa cytotoxicity was assessed using the LDH release assay described by CytoTox96 (Promega G1780). HeLa cells were grown in Dulbecco’s Modified Eagle Medium (DMEM; Gibco) supplemented with 10\% Fetal Bovine Serum (FBS) at $37^{\circ} \mathrm{C}$ with $5 \% \mathrm{CO}_{2}$. When the HeLa cultures exhibited $70-80 \%$ confluence, halogenated phenazines were then diluted by DMEM (10\% FBS) at concentrations of 25, 50 and $100 \mu \mathrm{M}$ and added to HeLa cells. Triton X-100 (at 2\% v/v) was used as the positive control for maximum lactate dehydrogenase (LDH) activity in this assay (i.e., complete cell death) while "medium only" lanes served as negative control lanes (i.e., no cell death). DMSO was used as our vehicle control. HeLa cells were treated with compounds for 24 hours and then $50 \mu \mathrm{L}$ of the supernatant was transferred into a fresh 96-well plate where $50 \mu \mathrm{L}$ of the reaction mixture was added to the 96-well plate and incubated at room temperature for 30 minutes. Finally, Stop Solution $(50 \mu \mathrm{L})$ was added to the incubating plates and the absorbance was measured at $490 \mathrm{~nm}$. Results are on the next page. 
Halogenated Phenazine cytotoxicity results (Triton-X $=100 \%$ cell death; Medium Only: $0 \%$ cell death):

2

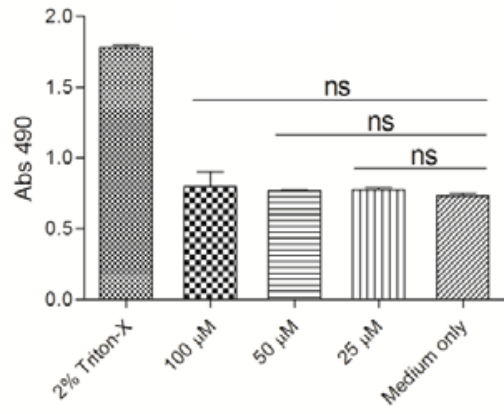

6

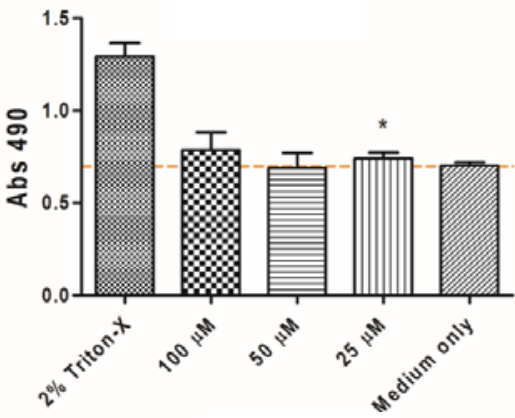

11

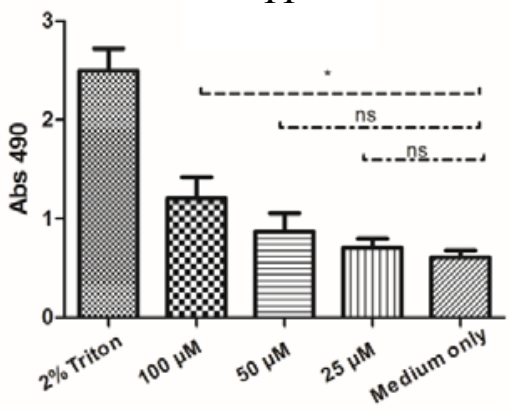

14
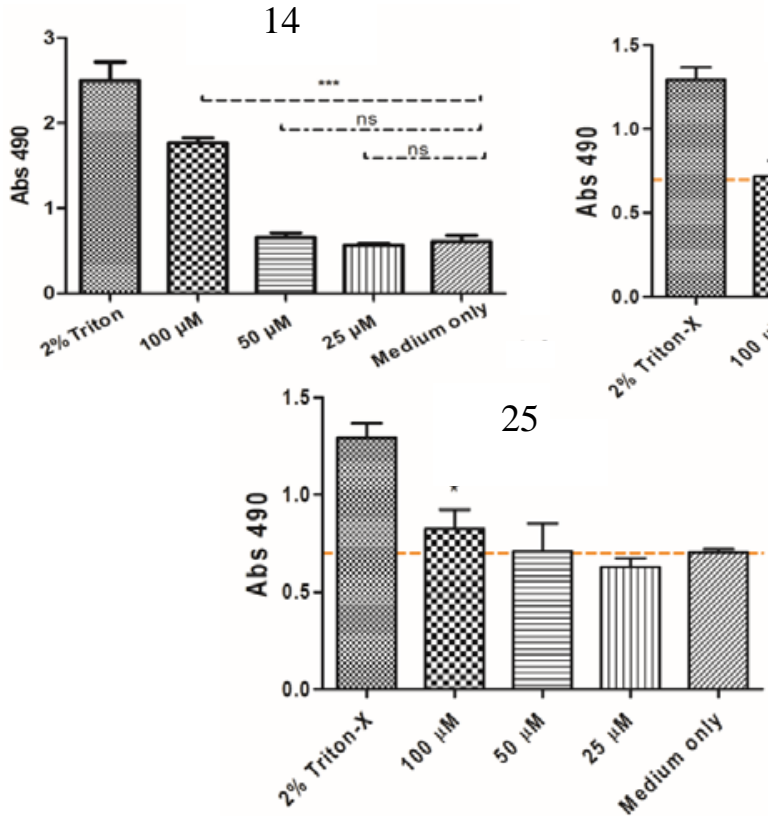

4

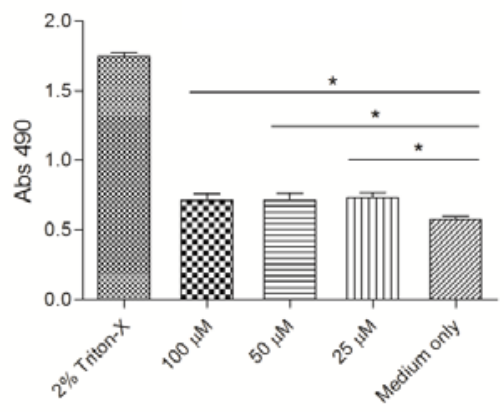

7

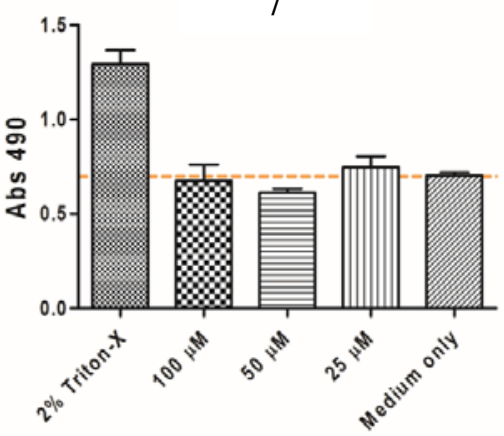

12

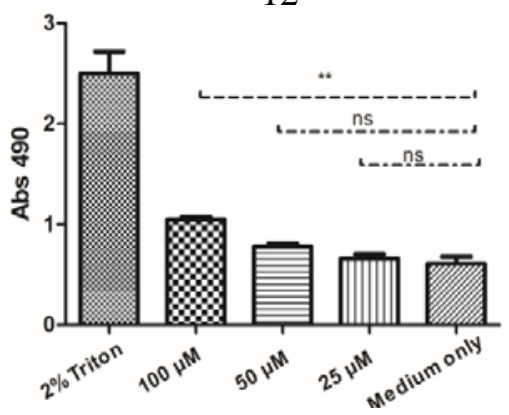

15

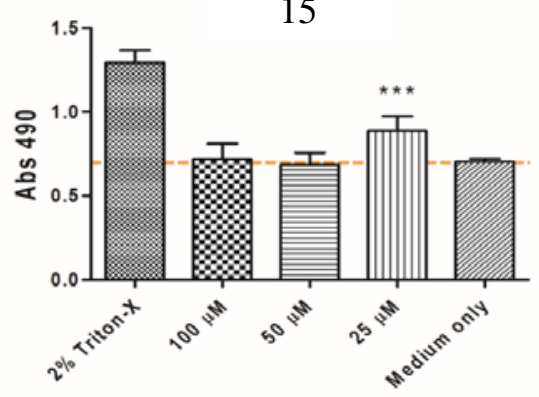

5

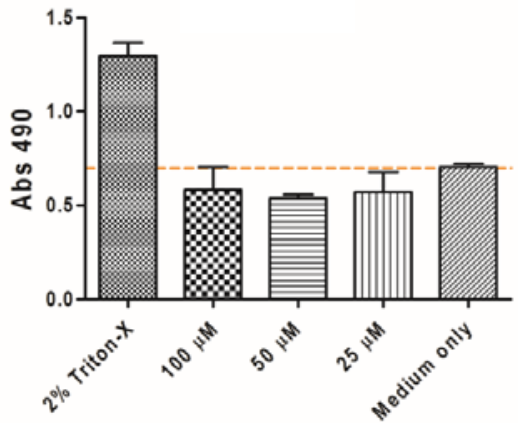

8

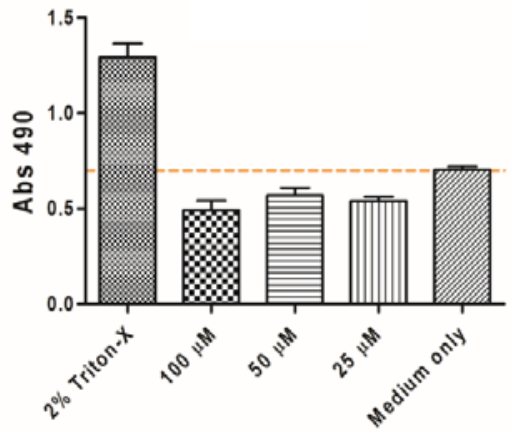

13

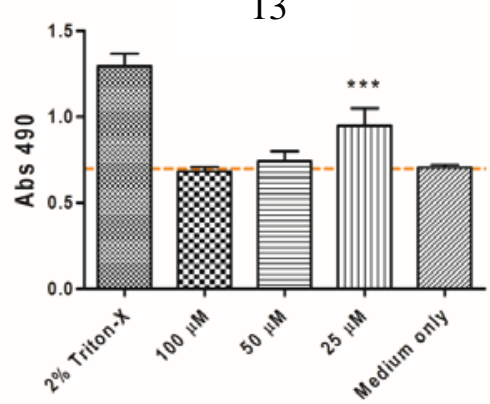

17

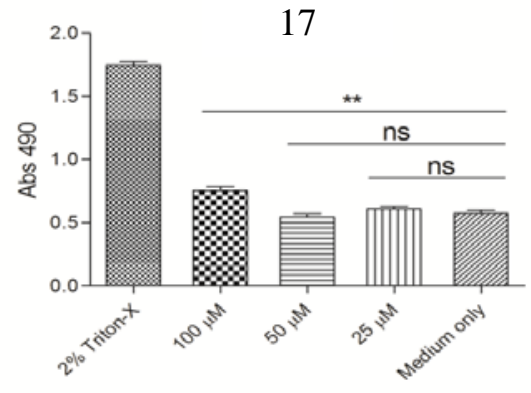

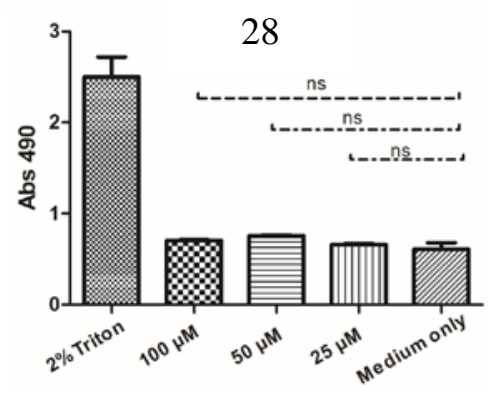




\section{4.) Literature References:}

1. Conda-Sheridan, M.; Marler, L.; Park, E. J.; Kondratyuk, T. P.; Jermihov, K.; Mesecar, A. D.; Pezzuto, J. M.; Asolkar, R. N.; Fenical, W.; Cushman, M. J. Med. Chem. 2010, 53, 8688-8699.

2. $\quad$ Chernetskii, V. P.; Kiprianov, A. I. Zh. Obshch. Khim., 1956, 26, 3032-3036.

3. Emmanuvel, L.; Shukla, R. K.; Sudalai, A.; Gurunath, S.; Sivaram, S. Tetrahedron Lett. 2006, 47, 4793-4796.

4. Clinical and Laboratory Standards Institute. 2009. Methods for dilution antimicrobial susceptibility tests for bacteria that grow aerobically; approved standard, 8th edition (M7-M8), Clinical and Laboratory Standard, Wayne, PA, 2009.

5. Abouelhassan, Y.; Garrison, A. T.; Bai, F.; Norwood IV, V. M.; Nguyen, M. T.; Jin, S.; Huigens III, R. W. ChemMedChem, 2015, 10, 1157-1162.

6. Garrison, A. T.; Abouelhassan, Y.; Kallifidas, D.; Bai, F.; Ukhanova, M.; Mai, V.; Jin, S.; Luesch, H.; Huigens III, R. W. Angew. Chem. Int. Ed., 2015, 54, 14819 -14823. 
5.) Supporting Images of Biological Experiments: 


\section{Biofilm Eradication against MRSA-2}

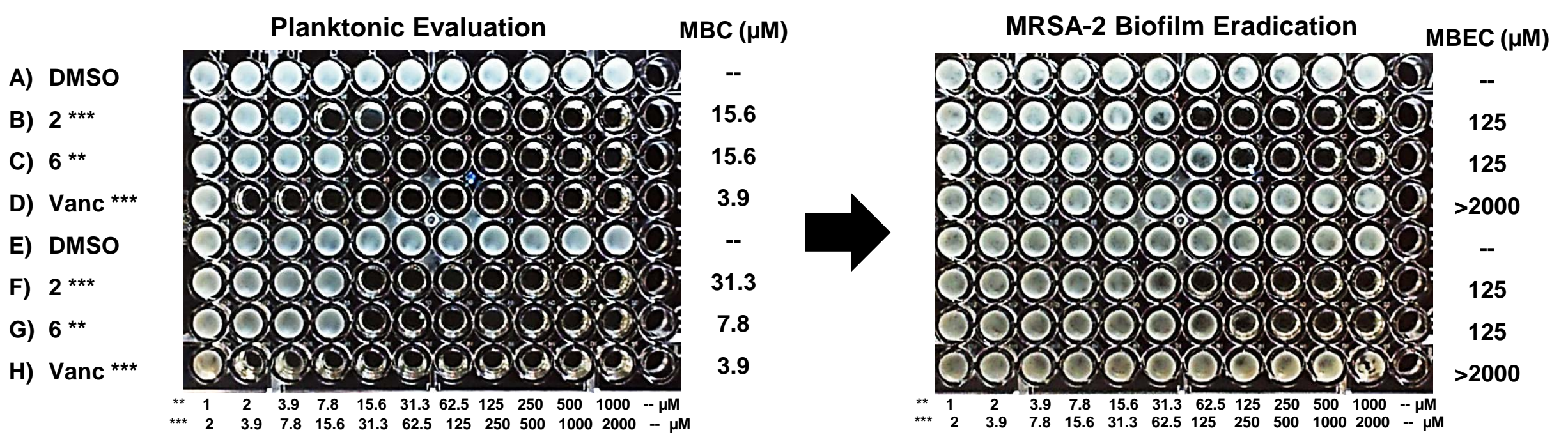

$\mathrm{MBC}(\mu \mathrm{M})$

15.6

15.6

3.9

7.8

$\begin{array}{lllllllllllll}* * * & 2 & 3.9 & 7.8 & 15.6 & 31.3 & 62.5 & 125 & 250 & 500 & 1000 & 2000 & --\mu M\end{array}$

$\operatorname{MBEC}(\mu \mathrm{M})$

125

125

$>2000$
Planktonic Evaluation

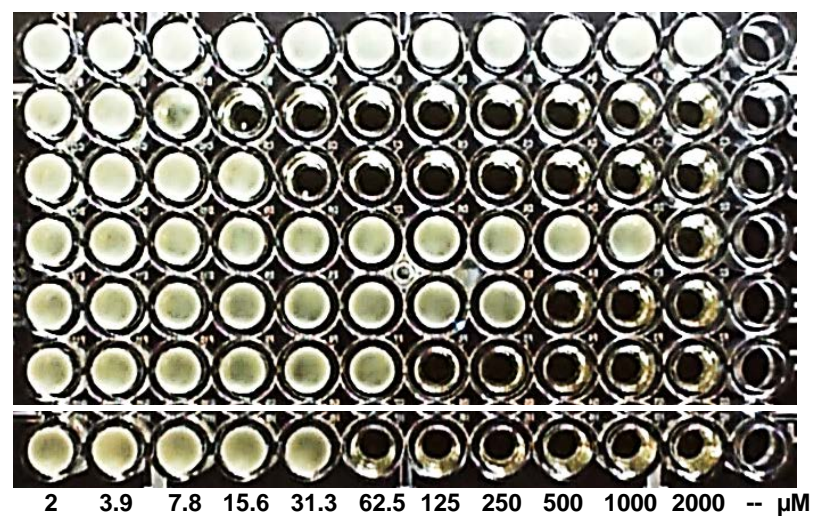

$\operatorname{MBC}(\mu \mathrm{M})$
A) DMSO
B) 2
C) 10
D) 26
E) 25
F) 27
G) 9

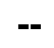

15.6

31.3

2000

500

125

62.5
MRSA-2 Biofilm Eradication

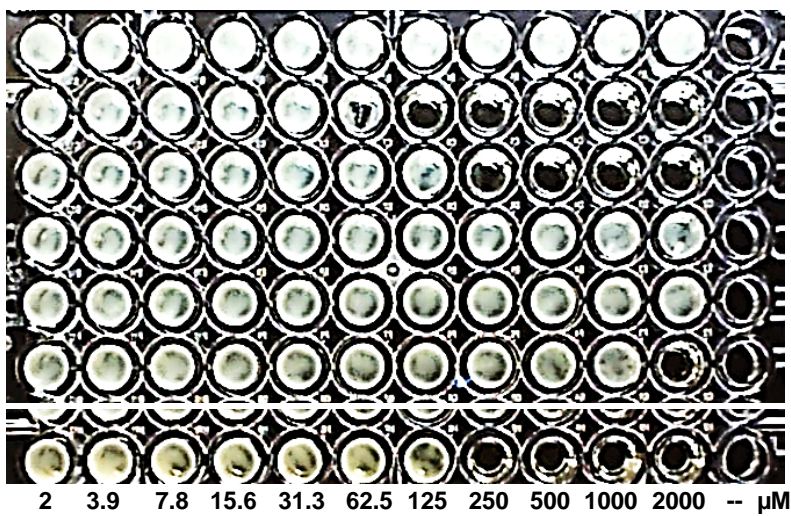

$\operatorname{MBEC}(\mu \mathrm{M})$

125

250

$>2000$

$>2000$

2000

250 


\section{Biofilm Eradication against MRSA-2}

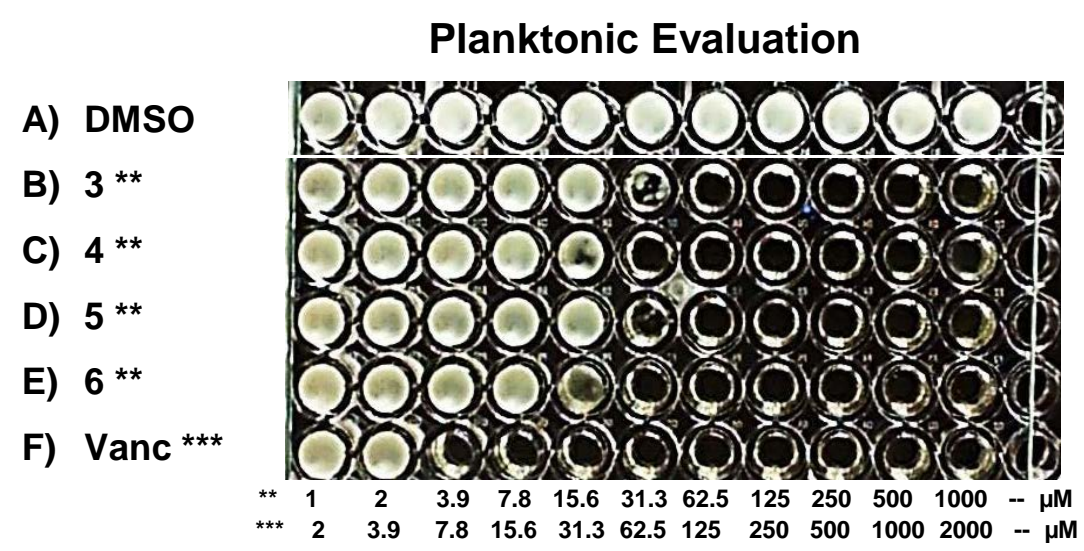

$\operatorname{MBC}(\mu \mathrm{M})$

MRSA-2 Biofilm Eradication

$-$

62.5

31.3

62.5

31.3

3.9
$\operatorname{MBEC}(\mu \mathrm{M})$

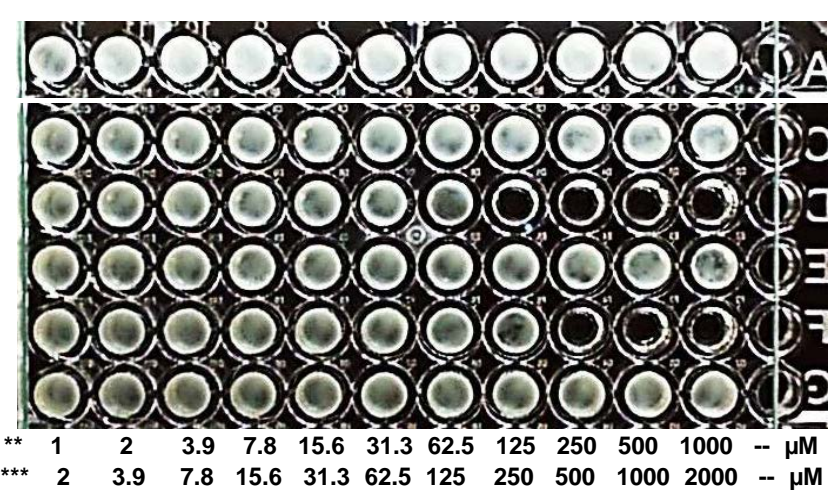

--

$>1000$

125

$>1000$

250

$>2000$
Planktonic Evaluation

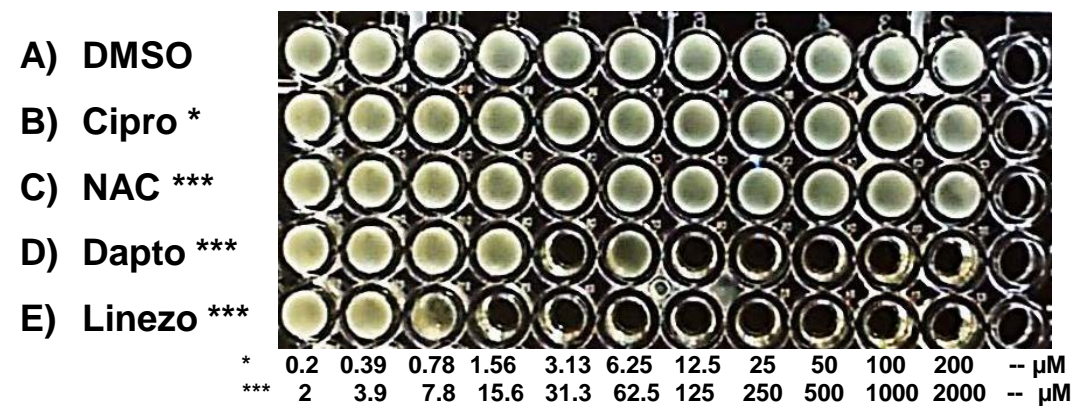

A) DMSO

B) Cipro *

D) Dapto **

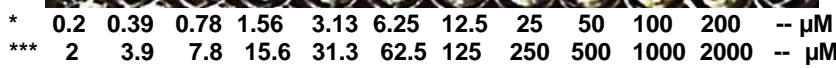

$\mathrm{MBC}(\mu \mathrm{M})$

$-$

$>200$

$>2000$

31.3

15.6
MRSA-2 Biofilm Eradication

$\operatorname{MBEC}(\mu \mathrm{M})$
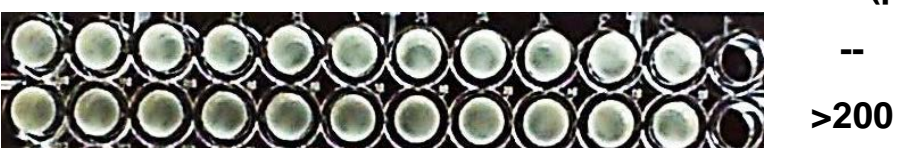

$>200$

$>2000$

$>2000$

$>2000$

Note: Vanc $=$ vancomycin, Cipro $=$ ciprofloxacin, NAC $=N$-acetylcysteine, Dapto $=$ daptomycin, Linezo $=$ linezolid 


\section{Biofilm Eradication against MRSA-2}

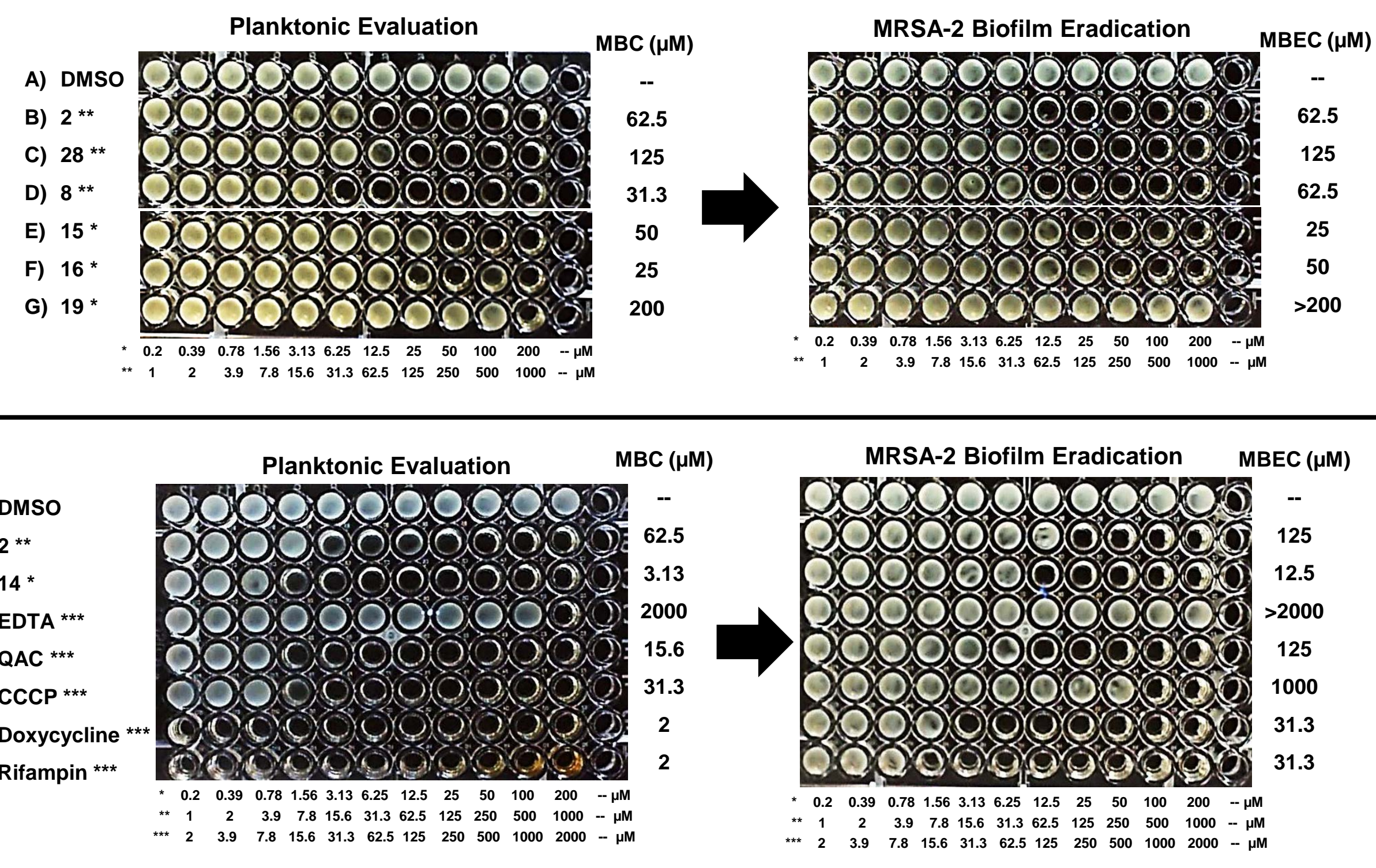




\section{Biofilm Eradication against MRSA BAA-1707}
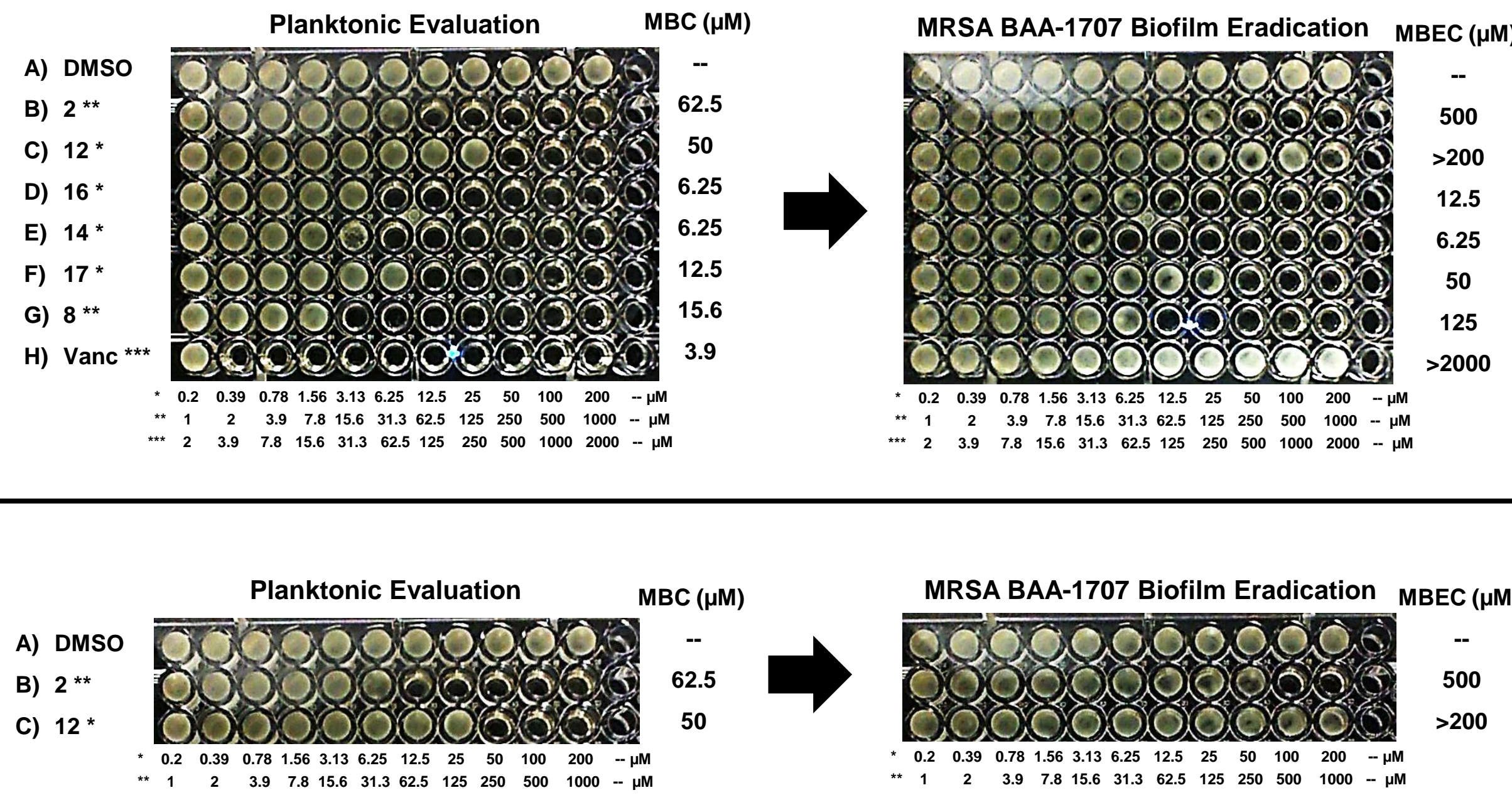

MBEC $(\mu \mathrm{M})$

$--$

500

$>200$

Note: Vanc = vancomycin 


\section{Biofilm Eradication against S. epidermidis (MRSE 35984)}

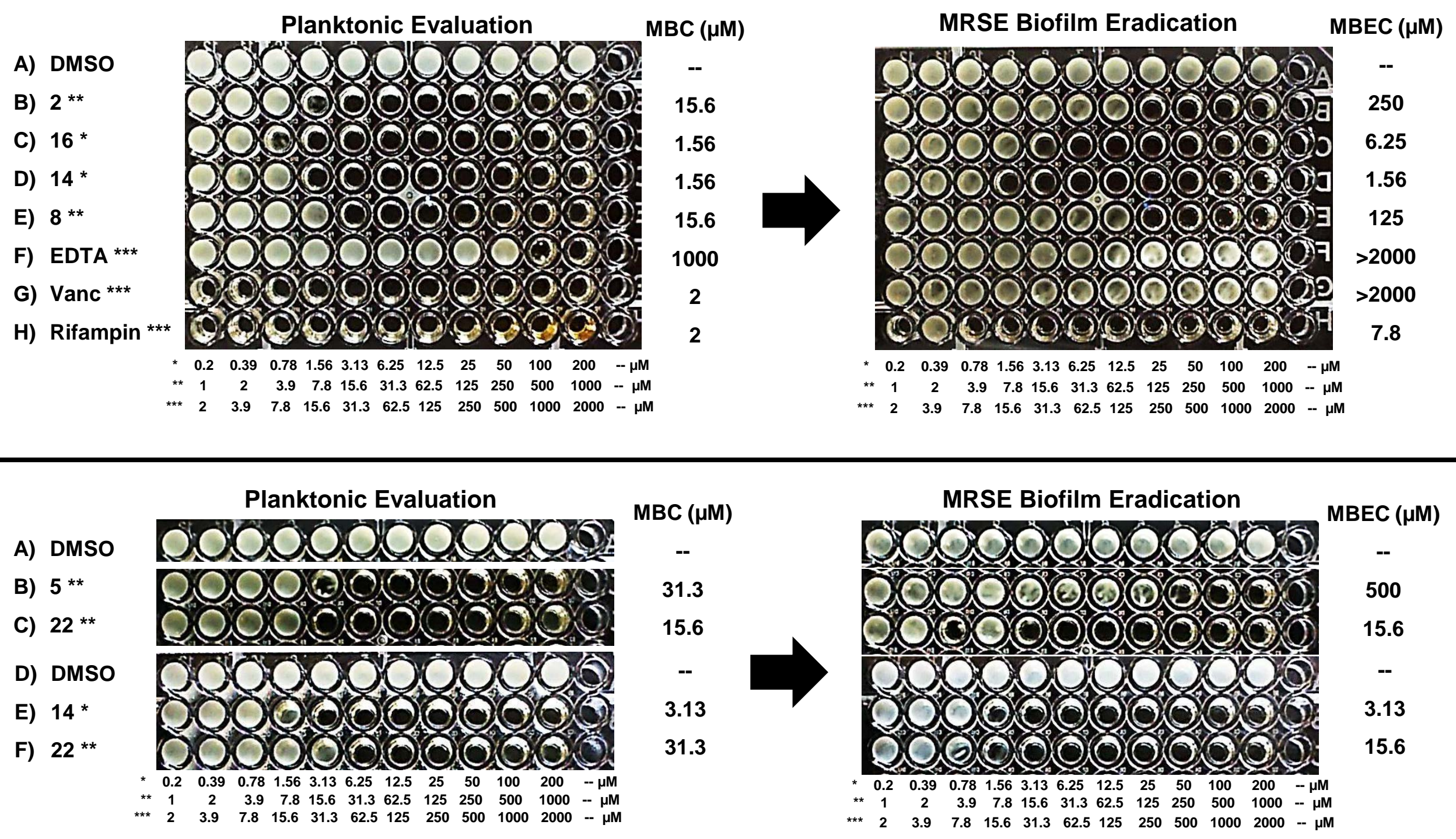




\section{Biofilm Eradication against E. faecium (VRE 700221)}

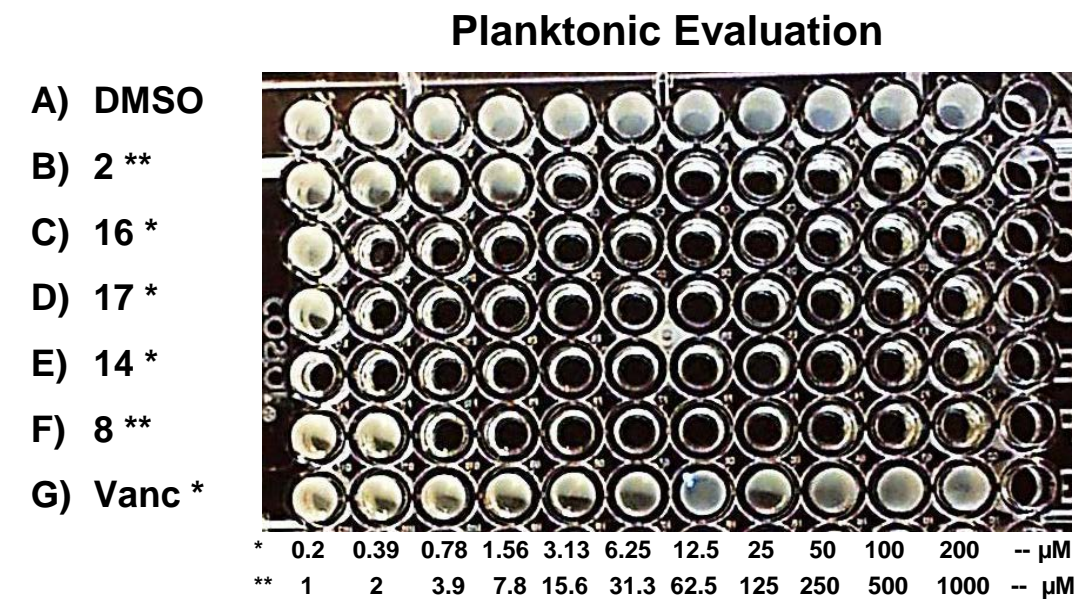

$\mathrm{MBC}(\mu \mathrm{M})$

-
15.6
0.39
0.39
0.2
2
$>200$

$\operatorname{MBEC}(\mu \mathrm{M})$

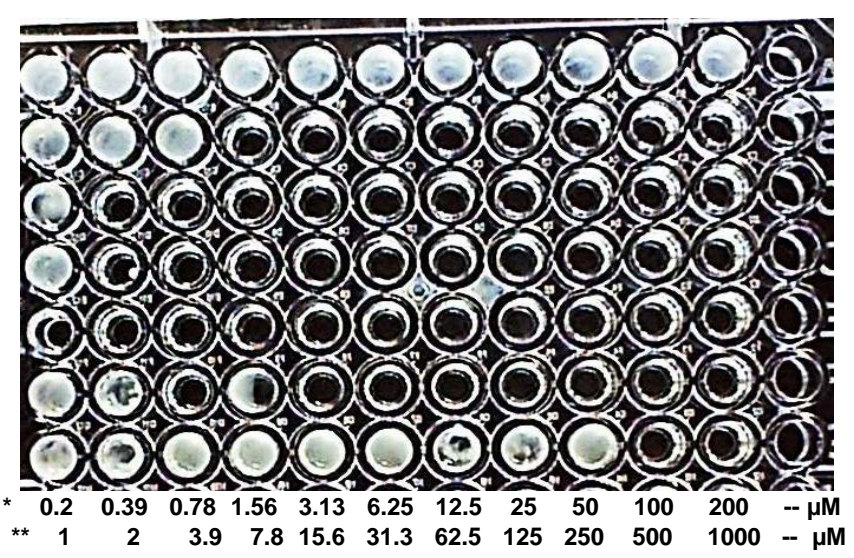

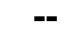

7.8

0.39

0.39

0.2

3.9

100

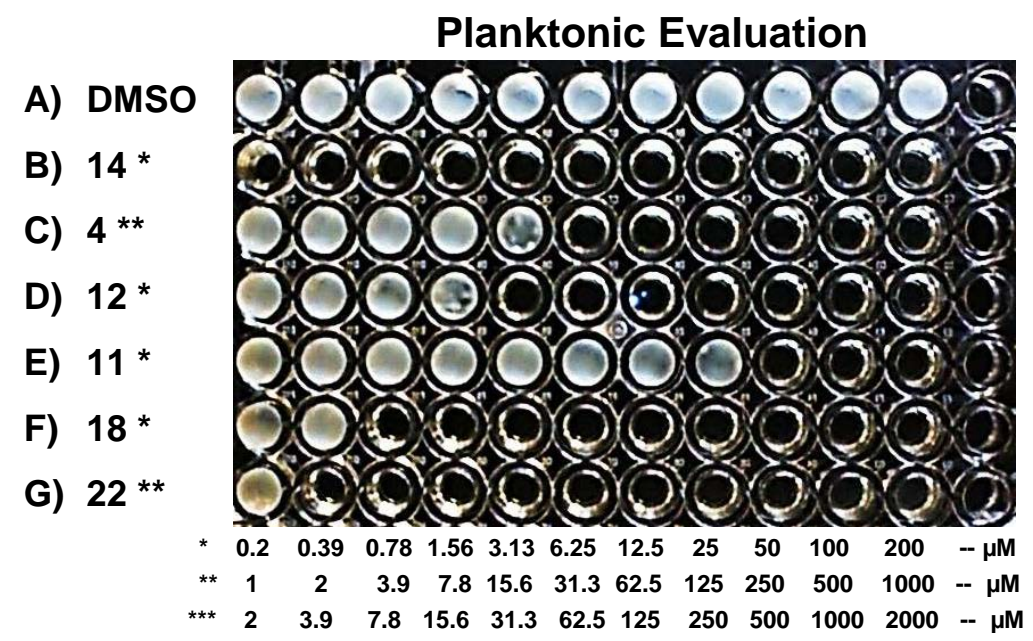

$\mathrm{MBC}(\mu \mathrm{M})$
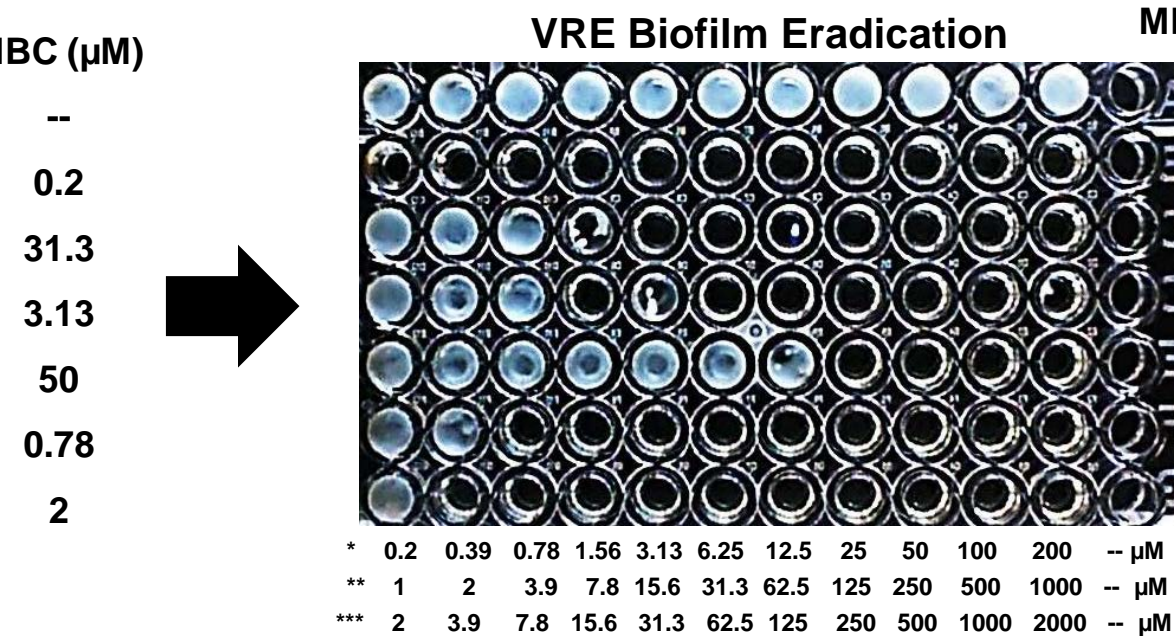

$\operatorname{MBEC}(\mu \mathrm{M})$

0.2

15.6

1.56

25

0.78

2

Note: Vanc $=$ vancomycin 


\section{MIC Assay against MRSA-2}

MIC against MRSA-2

A) DMSO

B) 2

C) 15

D) 10

E) 17

F) 8

G) 3
MIC $(\mu \mathrm{M})$

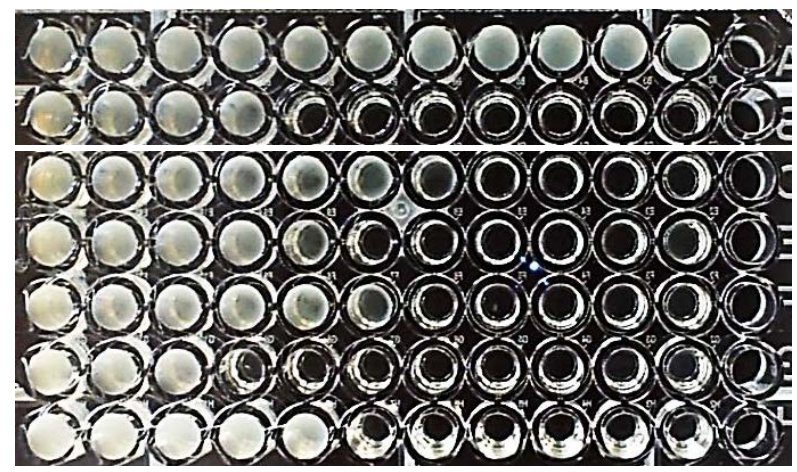

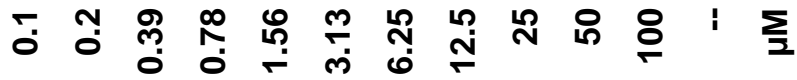

3.13

6.25

0.78

3.13
MIC against MRSA-2

A) DMSO

B) 2

C) 6

D) 4

E) 19

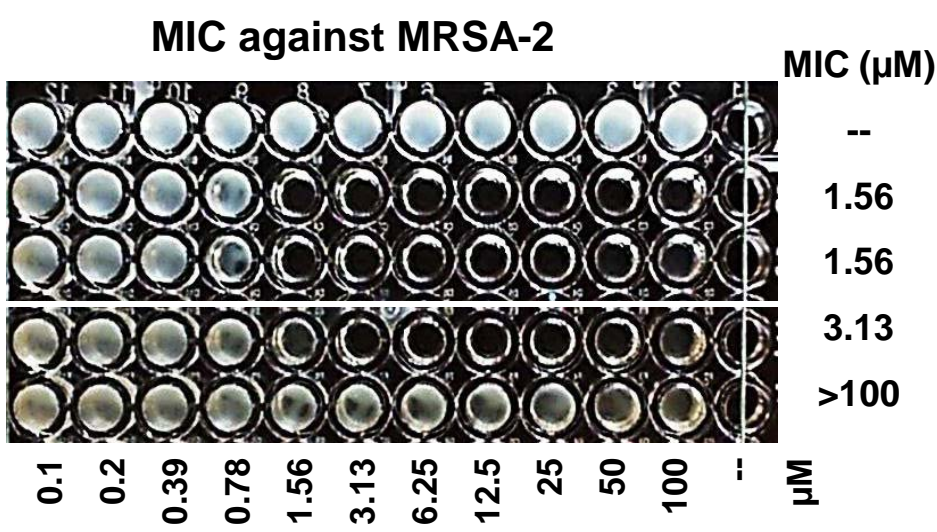

A) DMSO

B) 20

C) 2

D) 11

E) 21

F) 28

G) Linezolid

H) Daptomycin

\section{MIC against MRSA-2}

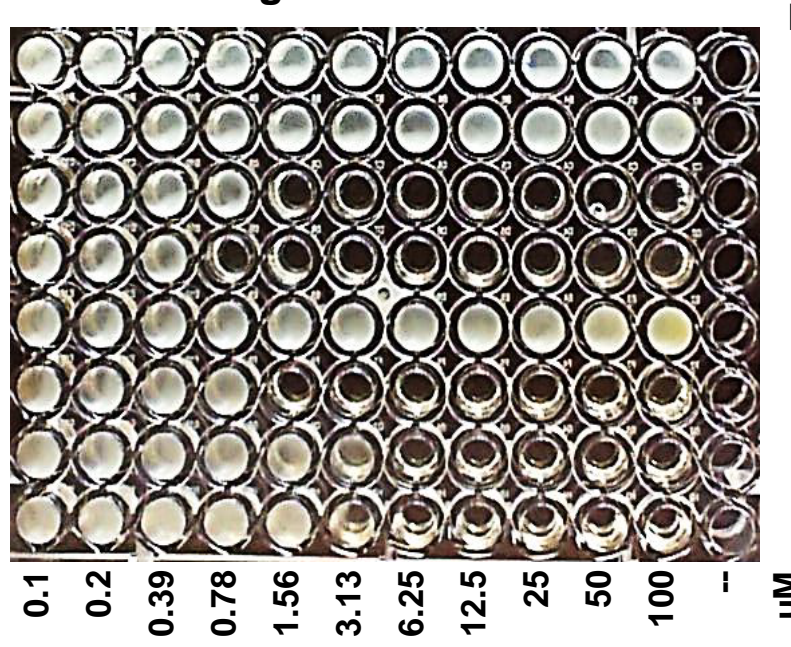

MIC $(\mu \mathrm{M})$

$-$

$>100$

1.56

0.78

$>100$

1.56

6.25

6.25
MIC against MRSA-2

A) DMSO

B) 5

C) 25

D) 27

E) 2

F) Vanc

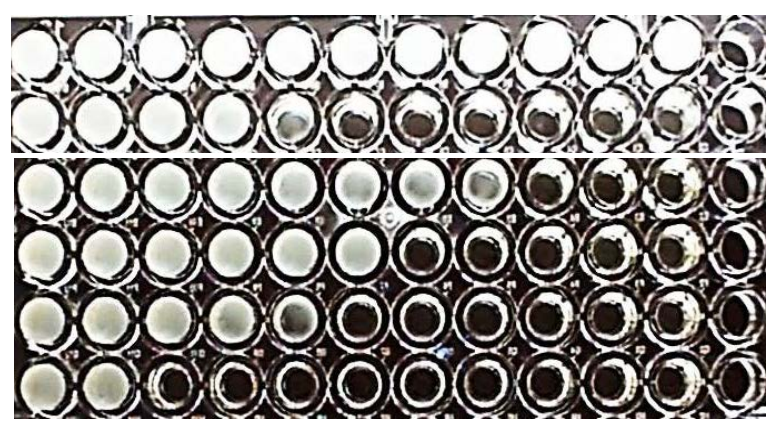

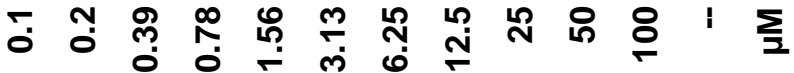

$\operatorname{MIC}(\mu \mathrm{M})$

-

3.13

25

6.25

3.13

0.39 


\section{MIC Assay against MRSA-2}
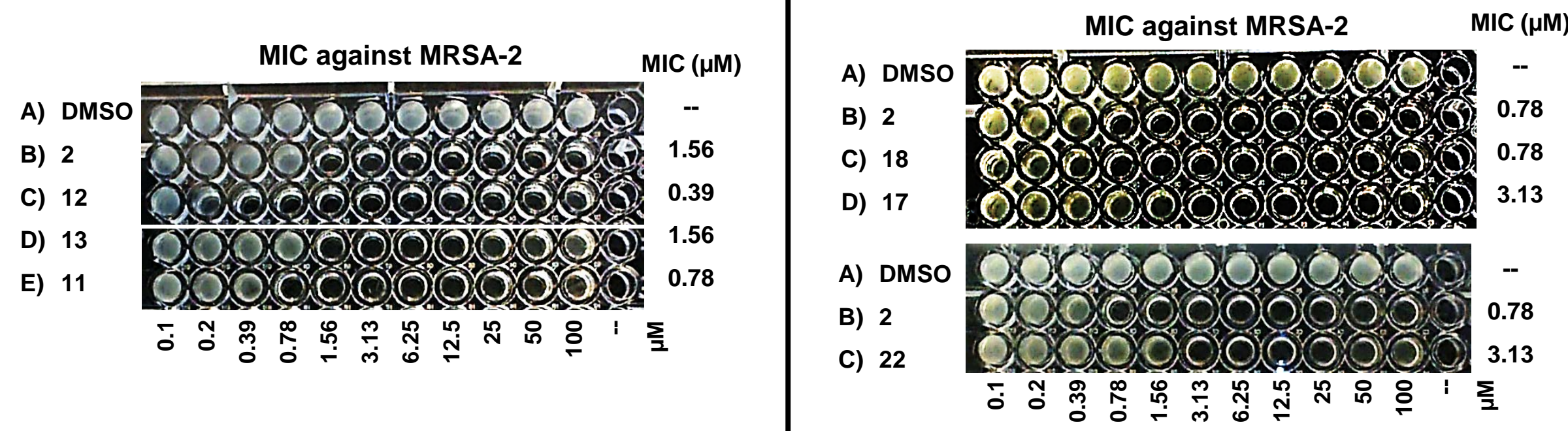


\section{MIC Assay against MRSA BAA-44 and BAA-1707}

A) DMSO

B) 2

C) 13

D) 18

E) 17

F) Daptomycin

G) Linezolid

H) Vancomycin

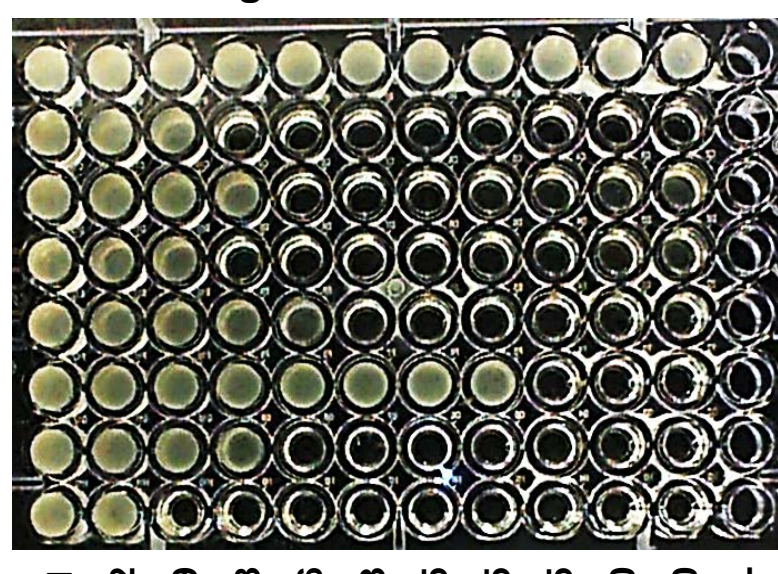

ㄴ.

$\operatorname{MIC}(\mu \mathrm{M})$

MIC against MRSA BAA-44

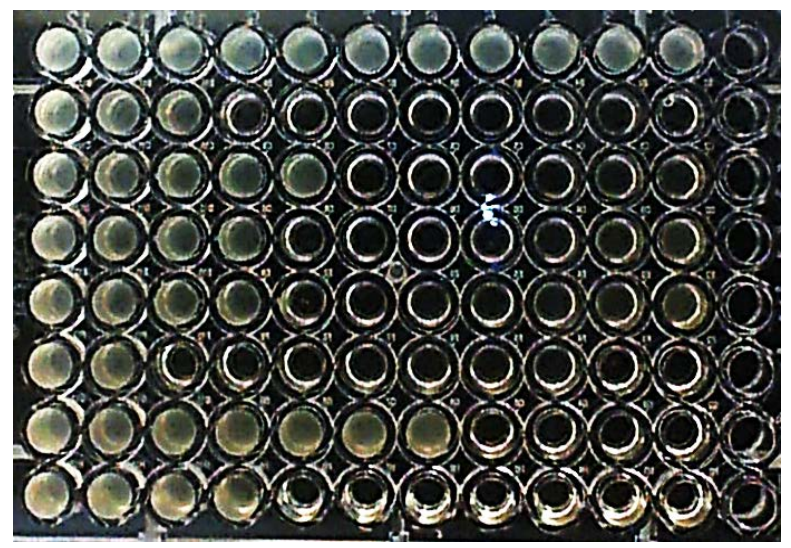

MIC ( $\mu \mathrm{M})$

\begin{tabular}{c|ll}
-- & A) & DMSO \\
0.78 & B) & 2 \\
1.56 & C) & 18 \\
0.78 & D) 22 \\
3.13 & E) 22 \\
25 & F) & Vancomycin \\
1.56 & G) & Daptomycin \\
0.39 & H) & Linezolid
\end{tabular}

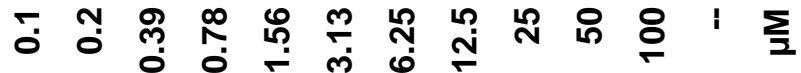

0.78

3.13

1.56

3.13

0.39

12.5

1.56

$\sum$

\section{MIC against MRSA BAA-1707 MIC $(\mu \mathrm{M})$}
A) DMSO
B) 2

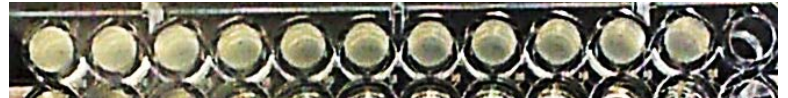
C) 13
D) 24
E) 18
F) 17
G) Linezolid
H) Daptomycin

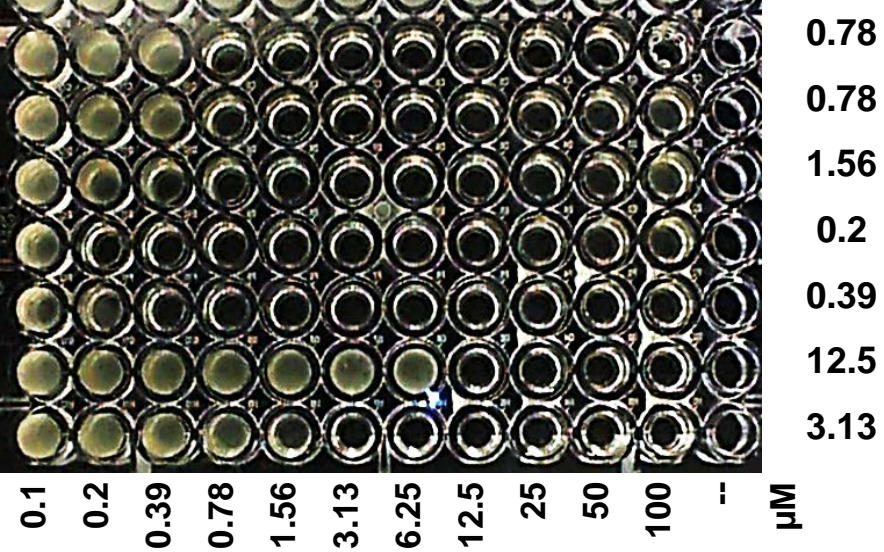




\section{MIC Assay against S. epidermidis (ATCC 12228)}

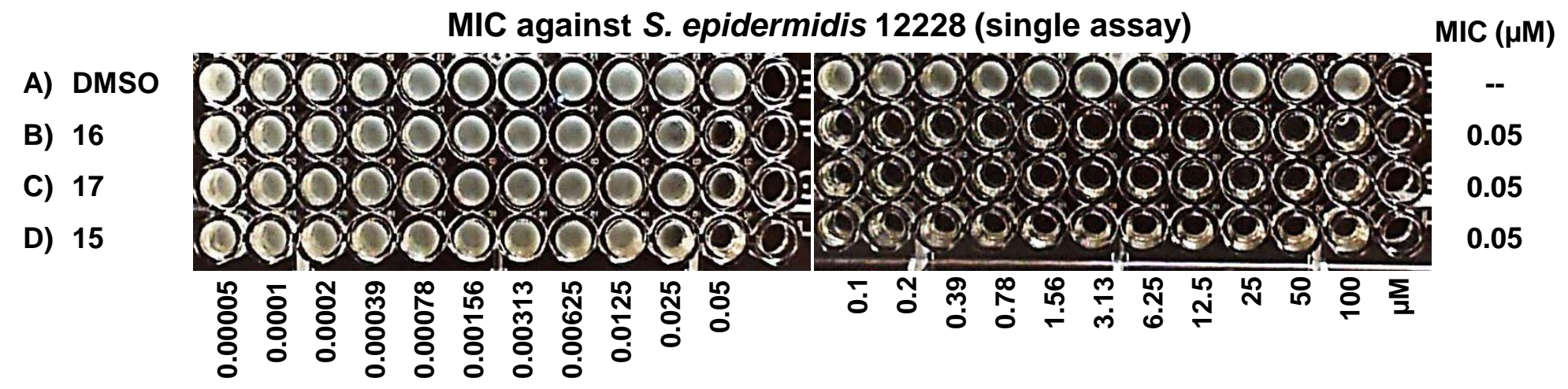

Note: MIC assays were done over two 96-well plates for highly potent compounds.

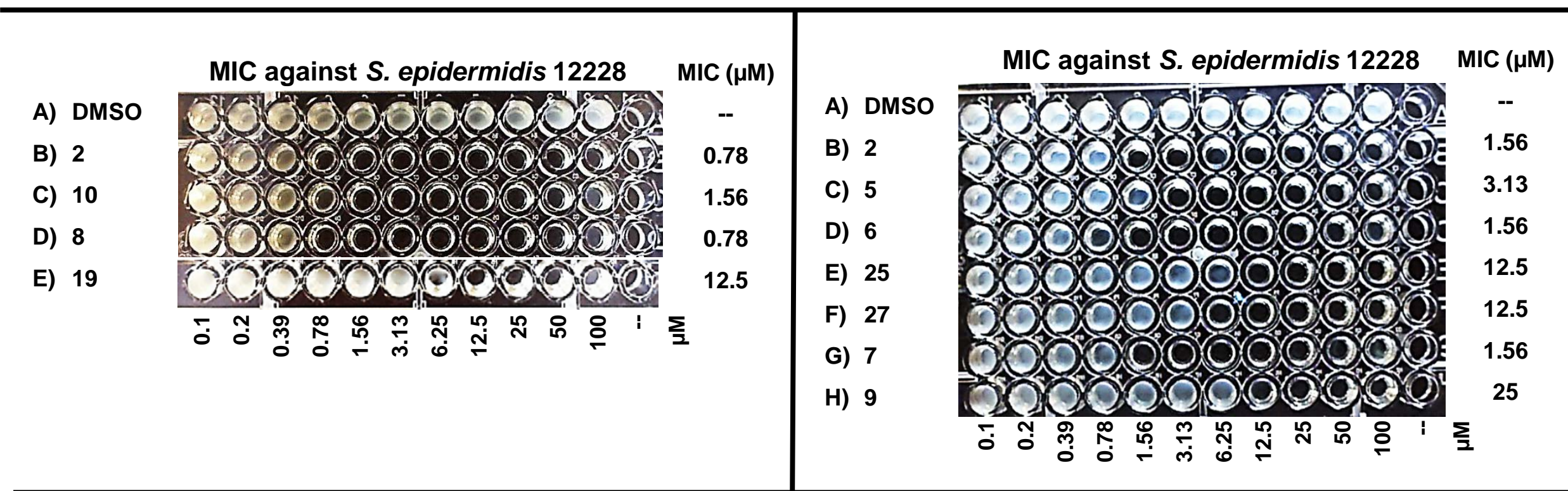




\section{MIC Assay against S. epidermidis (MRSE 35984)}

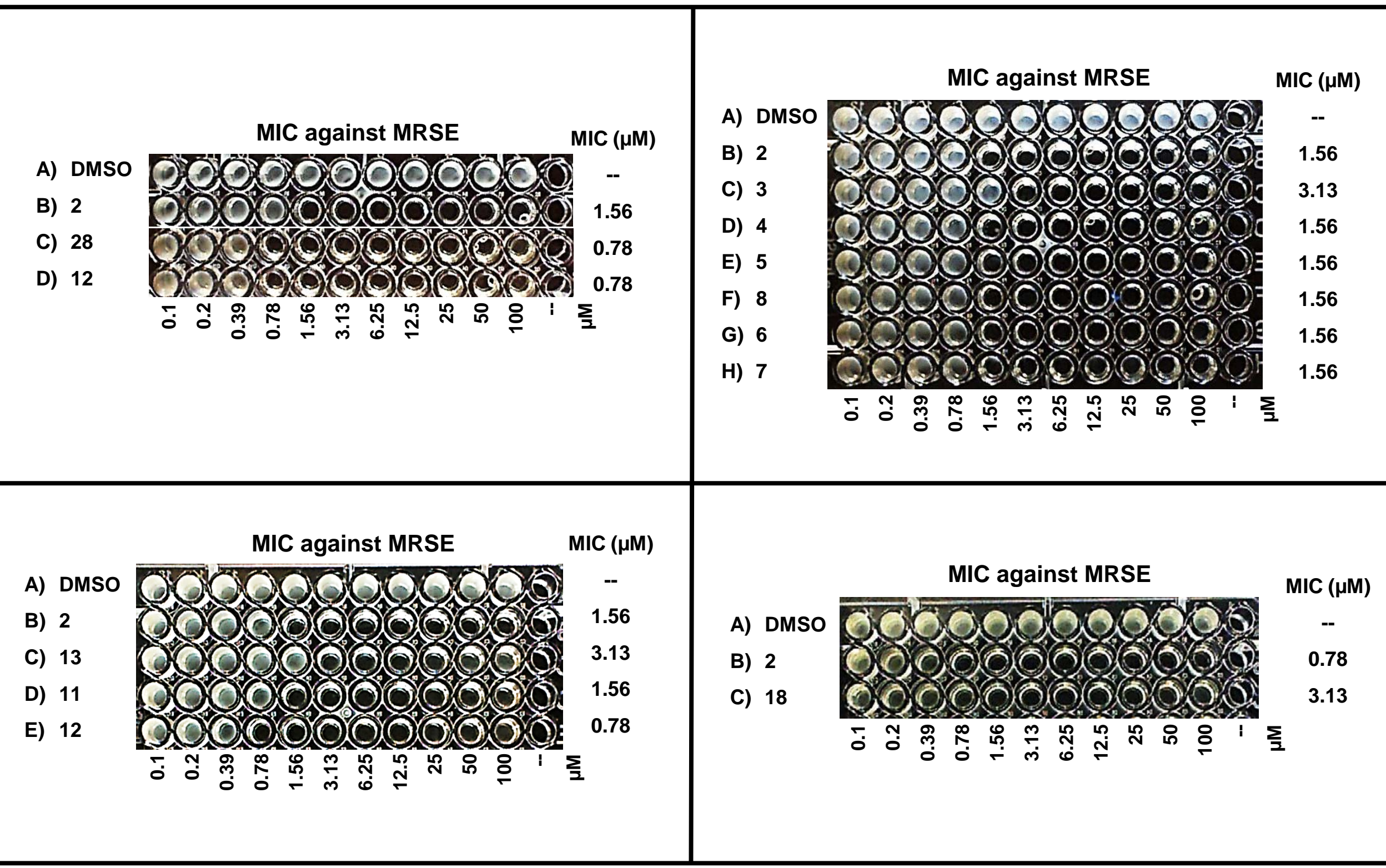




\section{MIC Assay against E. faecium (VRE 700221)}

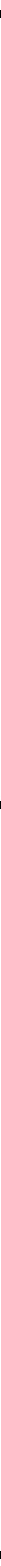




\section{Co-Treatment of MRSA-2 with Tiron and $\mathrm{CuSO}_{4}$}

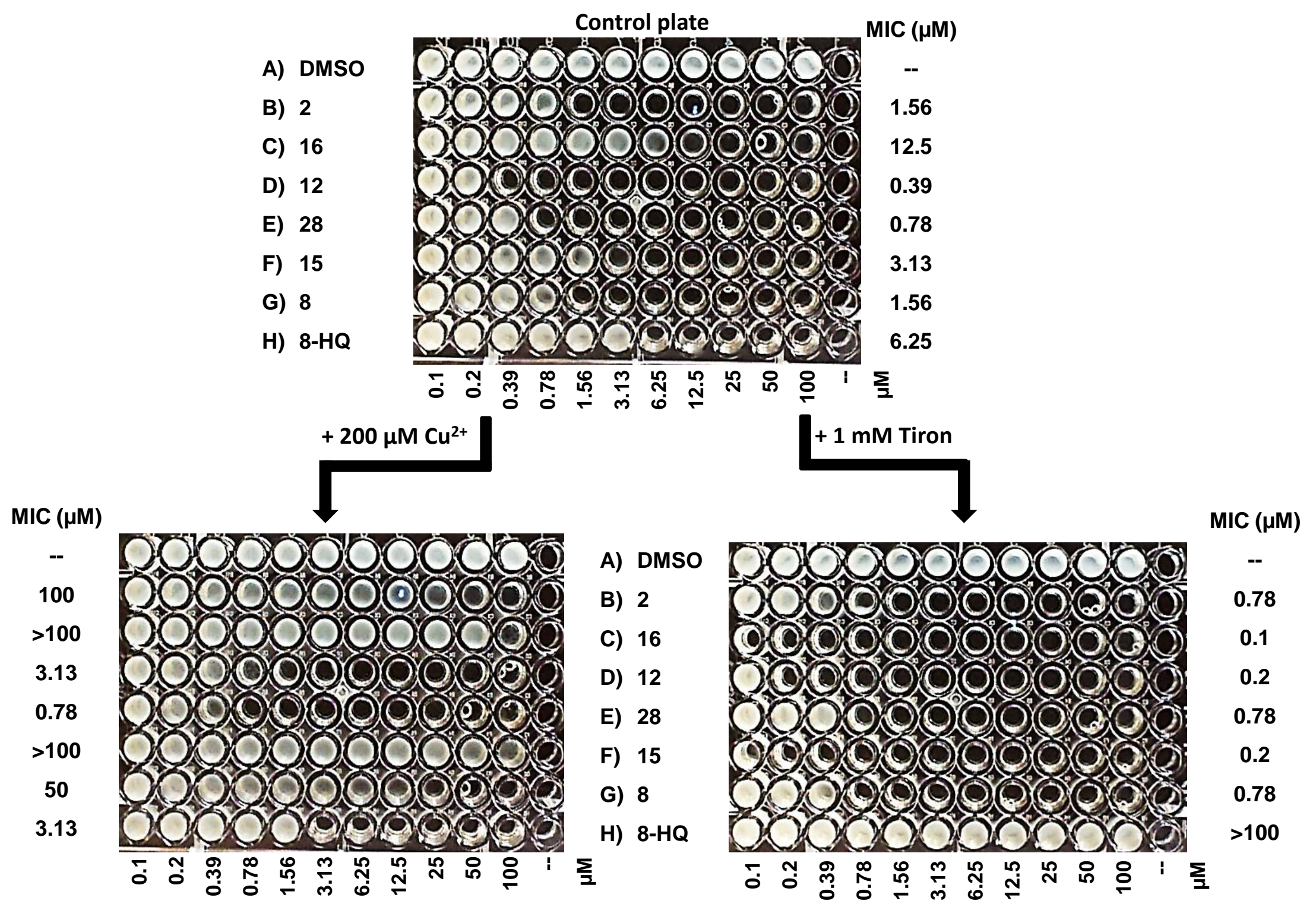




\section{Co-Treatment of S. epidermidis (ATCC 12228) with Tiron and $\mathrm{CuSO}_{4}$}

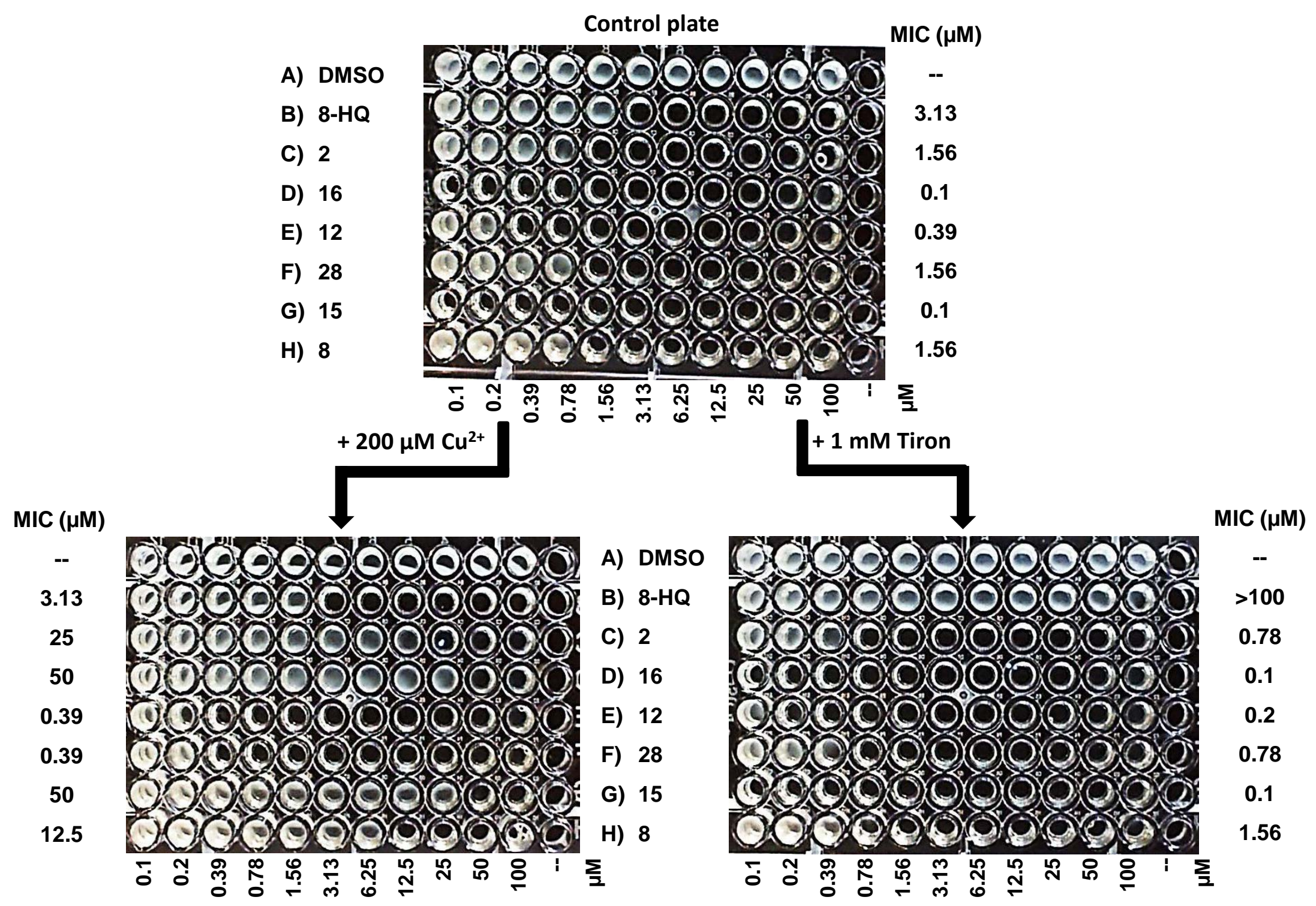




\section{Co-Treatment of E. faecium (VRE 700221) with Tiron and $\mathrm{CuSO}_{4}$}

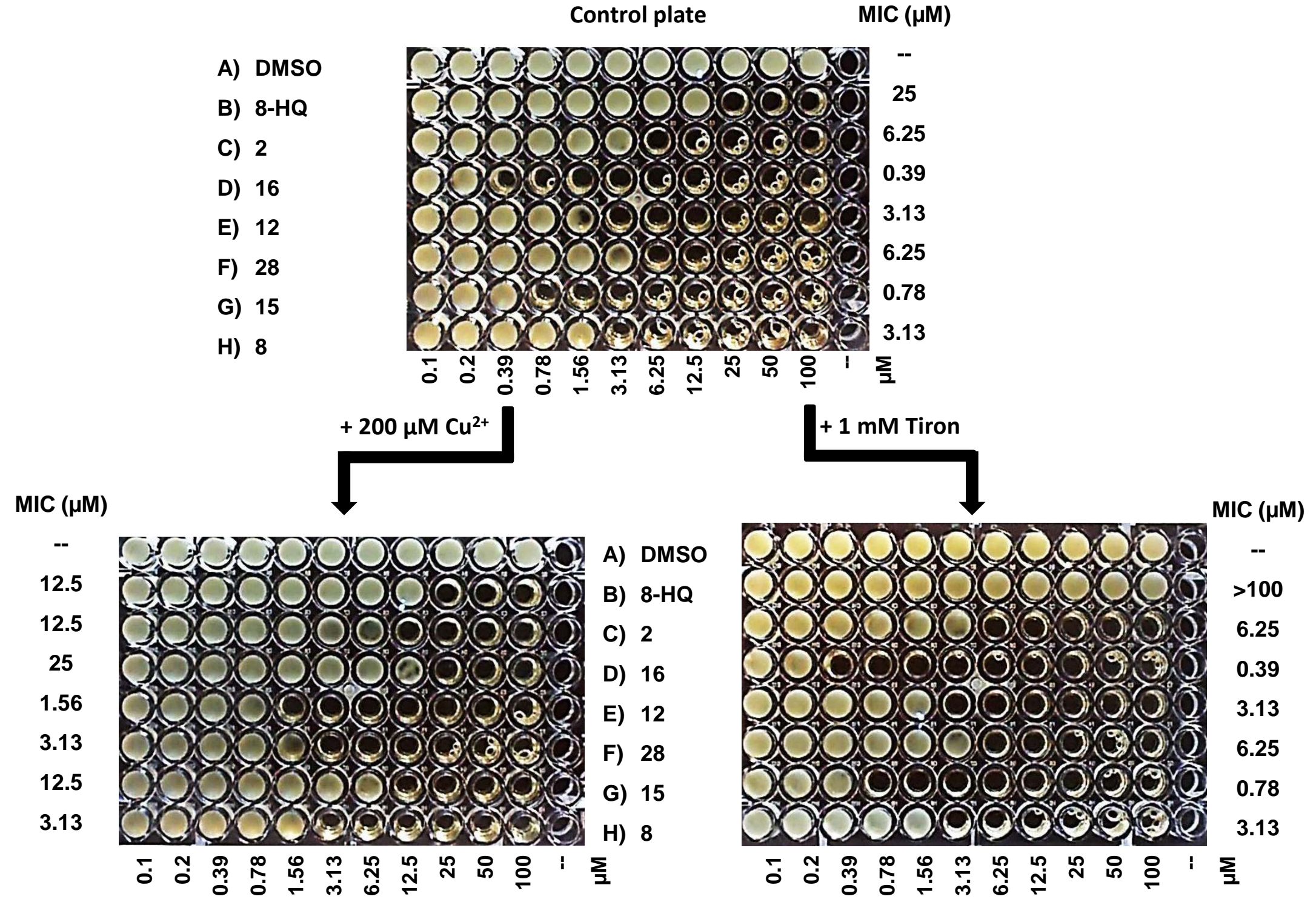


6. ${ }^{1} \mathrm{H}$ NMR \& ${ }^{13} \mathrm{C}$ NMR Spectra for New Compounds: 


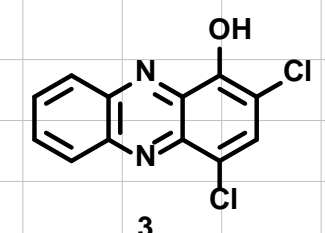

3

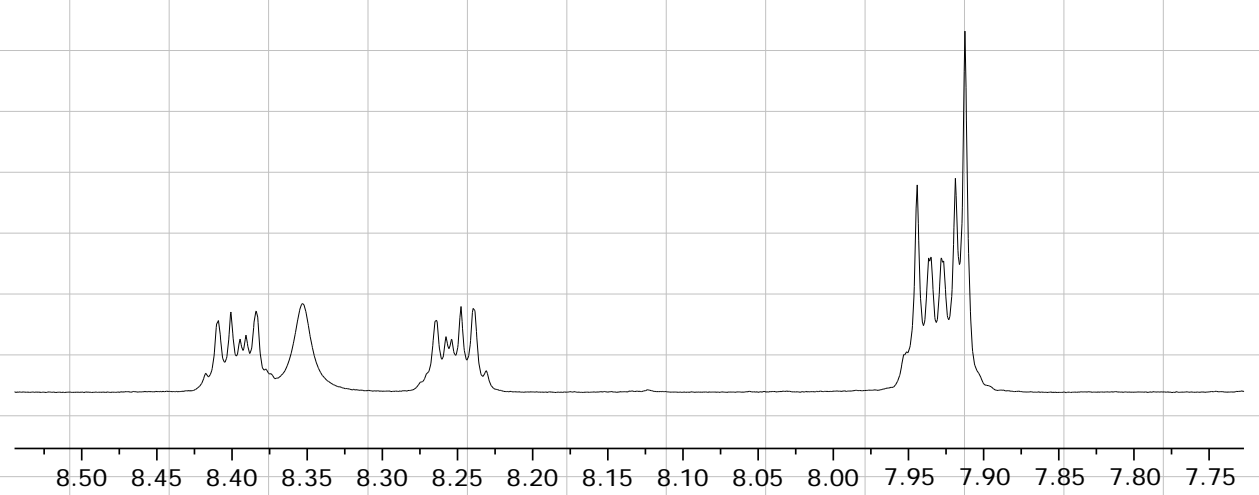

f1 (ppm) 


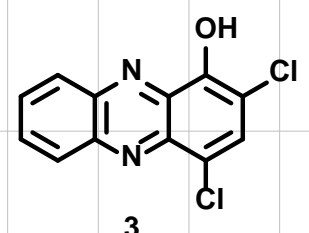

3

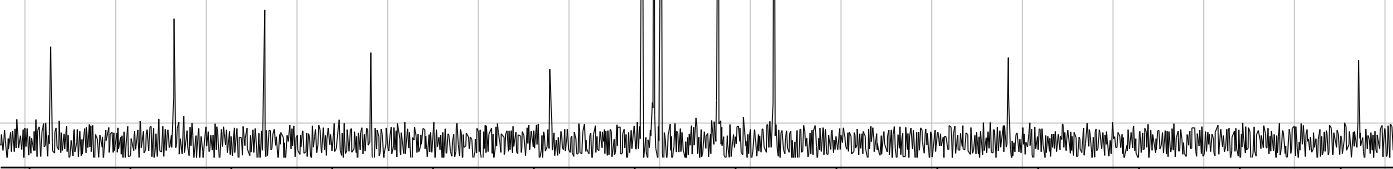

145

140

135

130

125

120

115

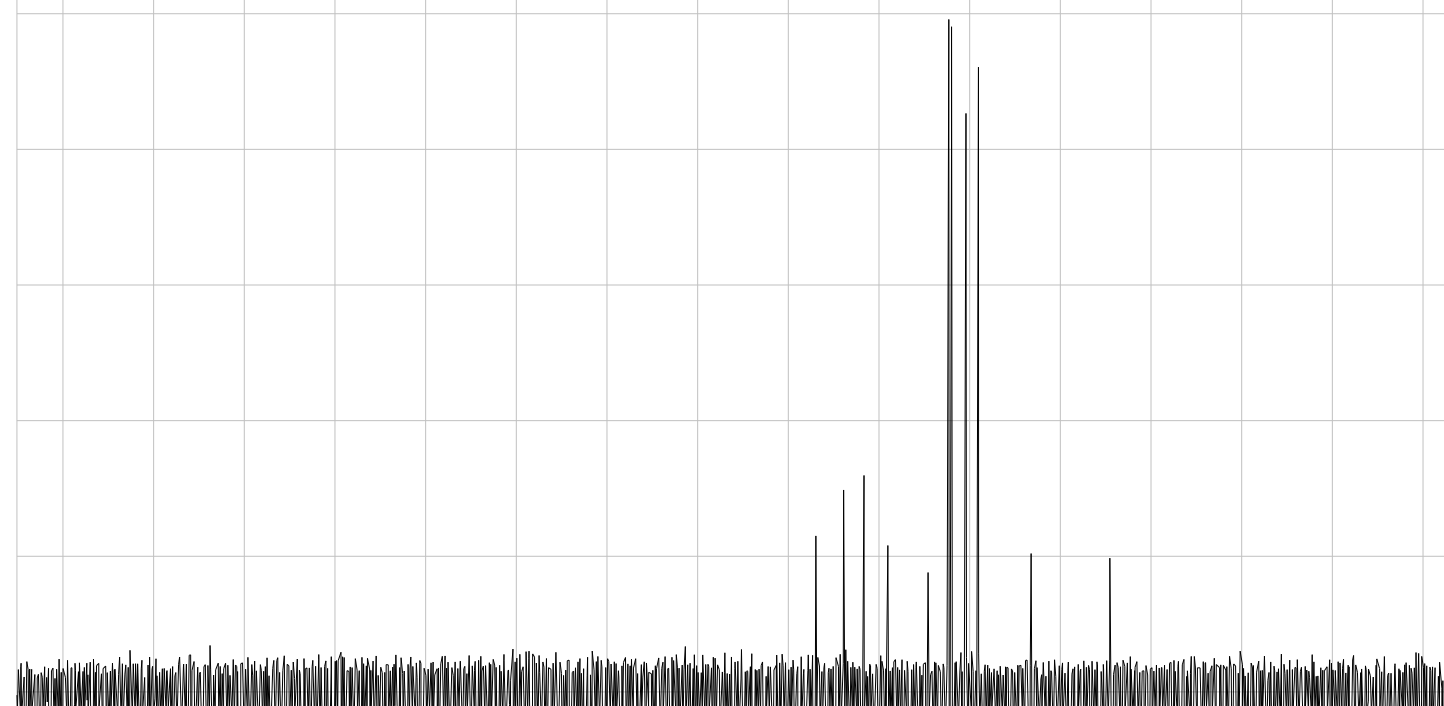

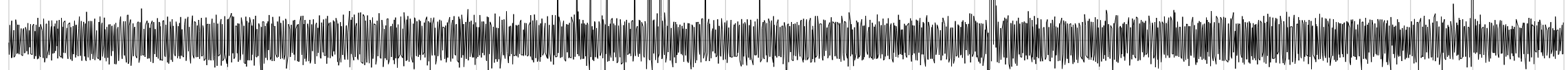

$\begin{array}{lllllllllllllllllllllllll}230 & 220 & 210 & 200 & 190 & 180 & 170 & 160 & 150 & 140 & 130 & 120 & \begin{array}{c}110 \\ \mathrm{f} 1(\mathrm{ppm})\end{array} & 100 & 90 & 80 & 70 & 60 & 50 & 40 & 30 & 20 & 10 & 0 & -10\end{array}$




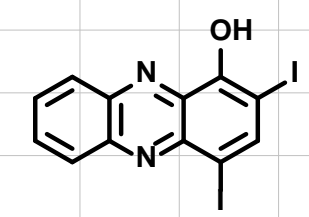

4

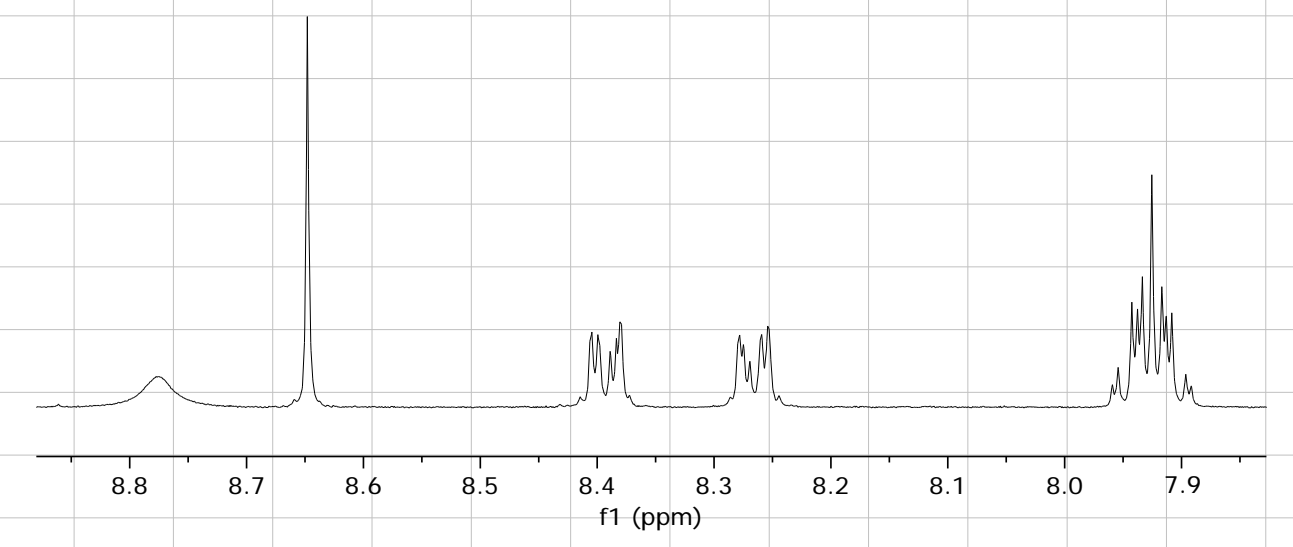


<smiles>Cc1cc(I)c(O)c2nc3ccccc3nc12</smiles>

4

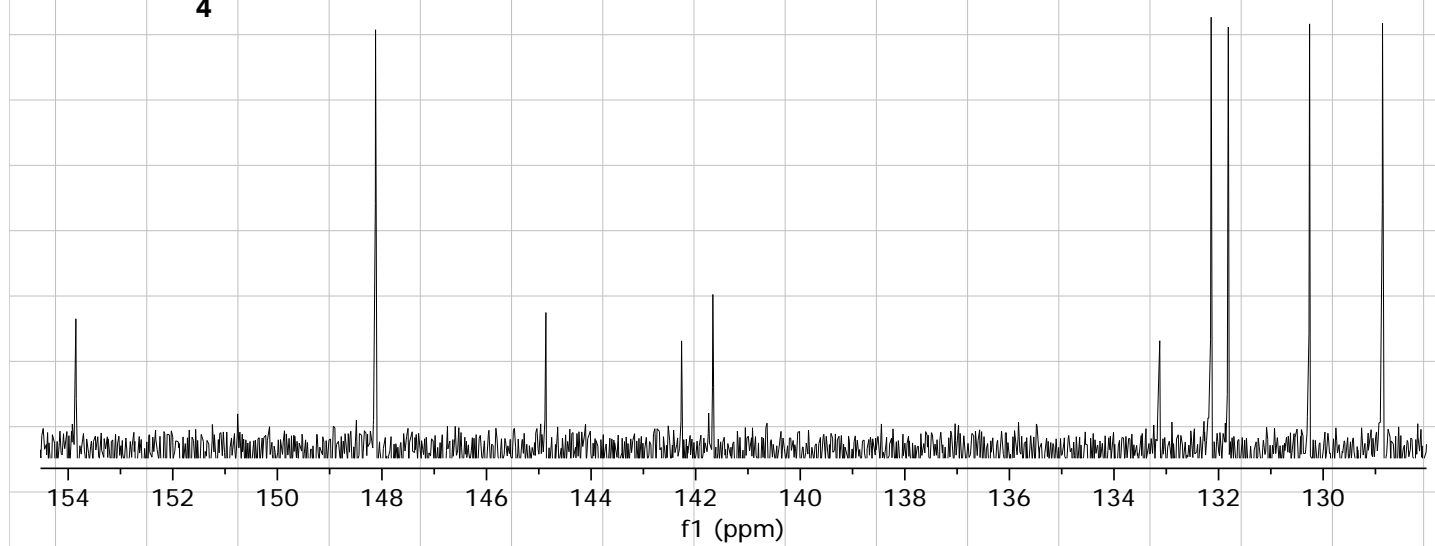

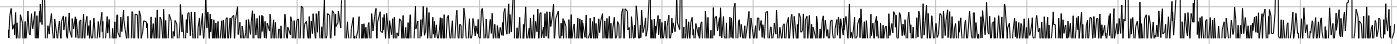
$\begin{array}{llllllll}154 & 152 & 150 & 148 & 146 & 144 & 142 & 140\end{array}$

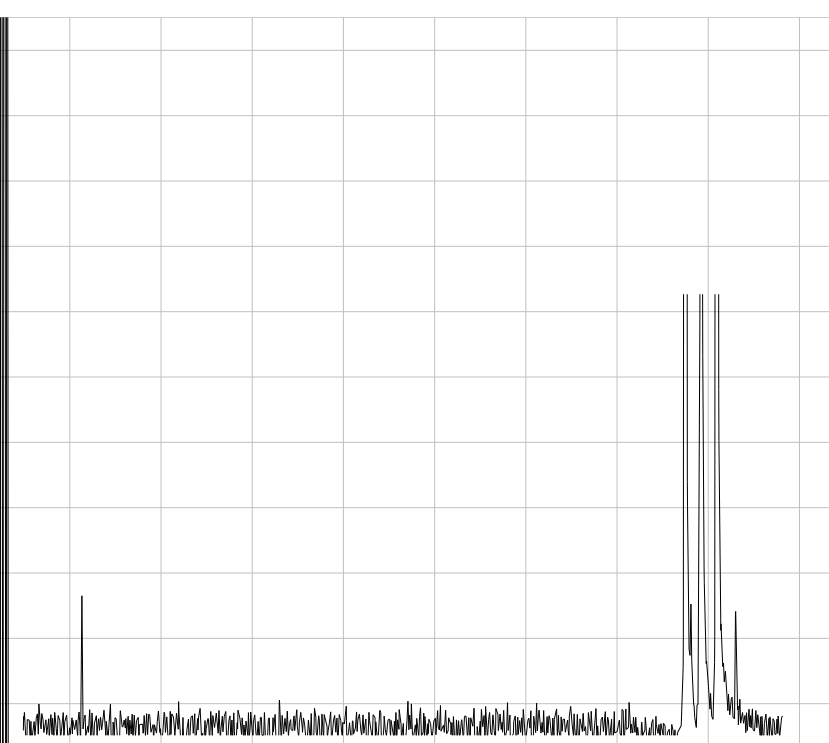

$\frac{1}{90}$

85

f1 (ppm)
80

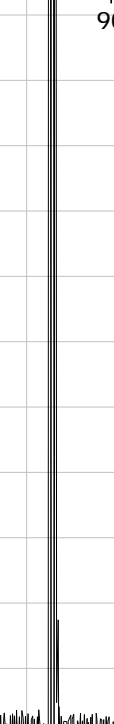

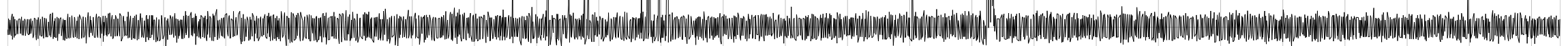

\begin{tabular}{llllllllllllllllllllllllll}
\hline 230 & 220 & 210 & 200 & 190 & 180 & 170 & 160 & 150 & 140 & 130 & 120 & 110 & 100 & 90 & 80 & 70 & 60 & 50 & 40 & 30 & 20 & 10 & 0 & -10
\end{tabular}



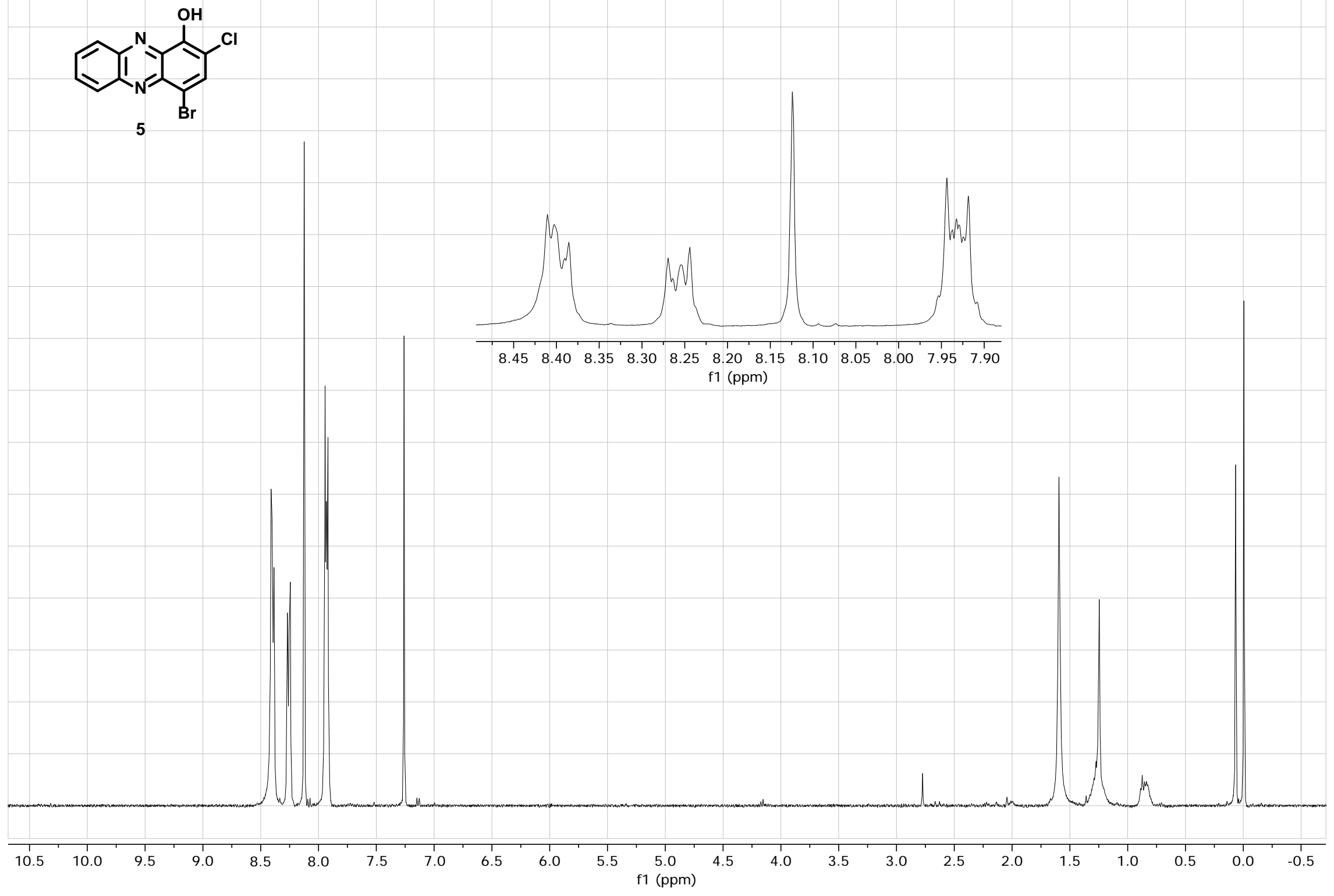


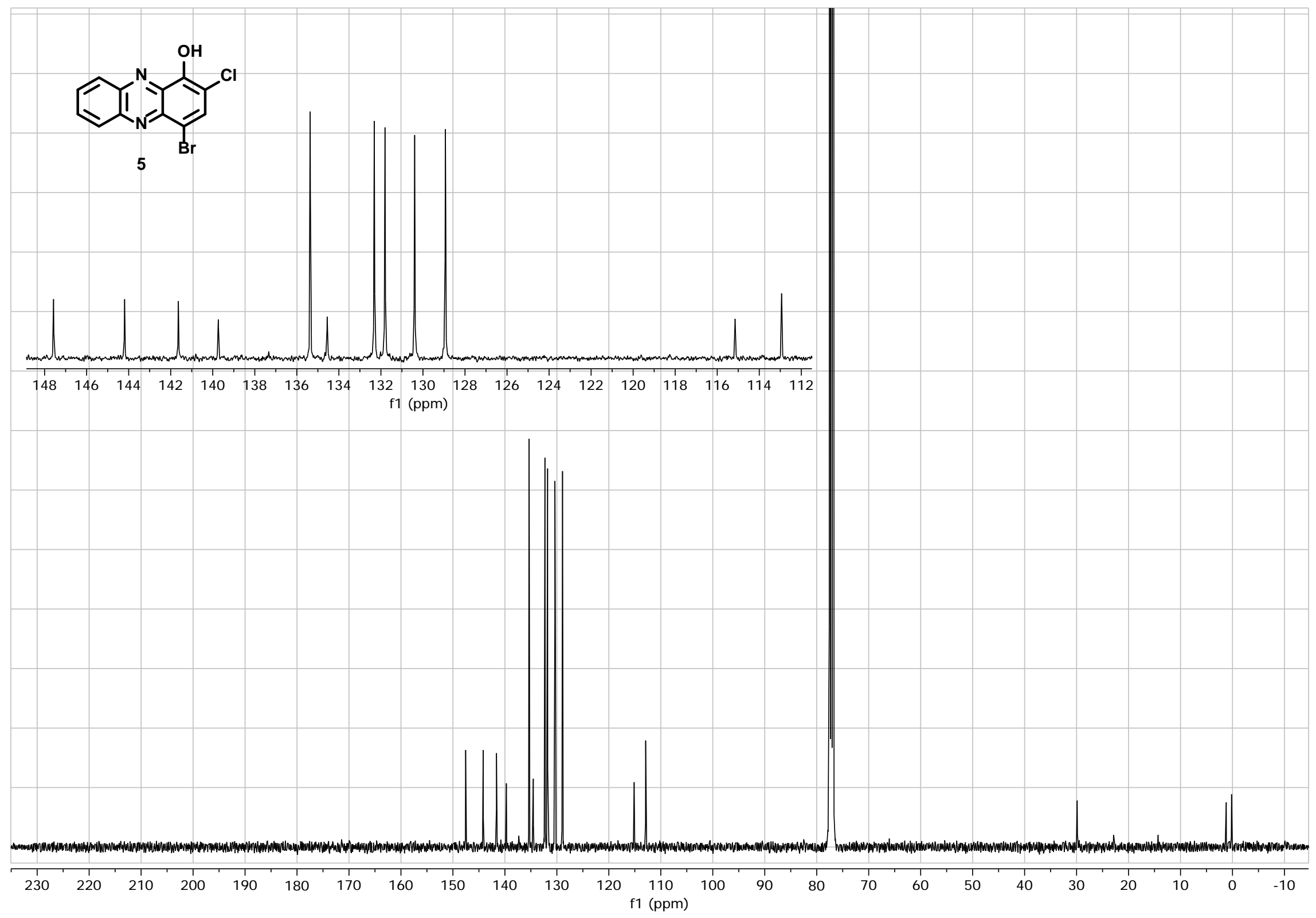




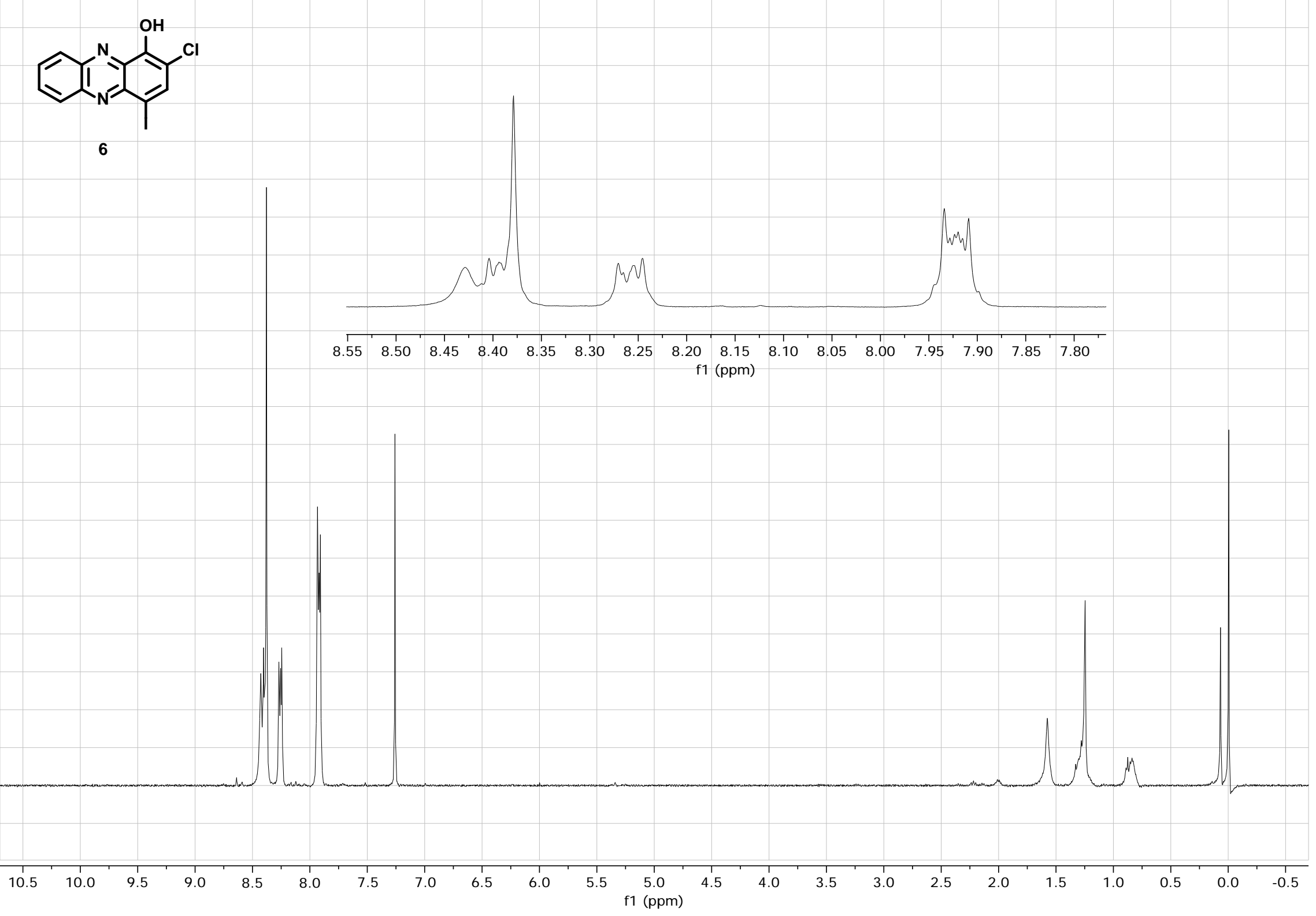




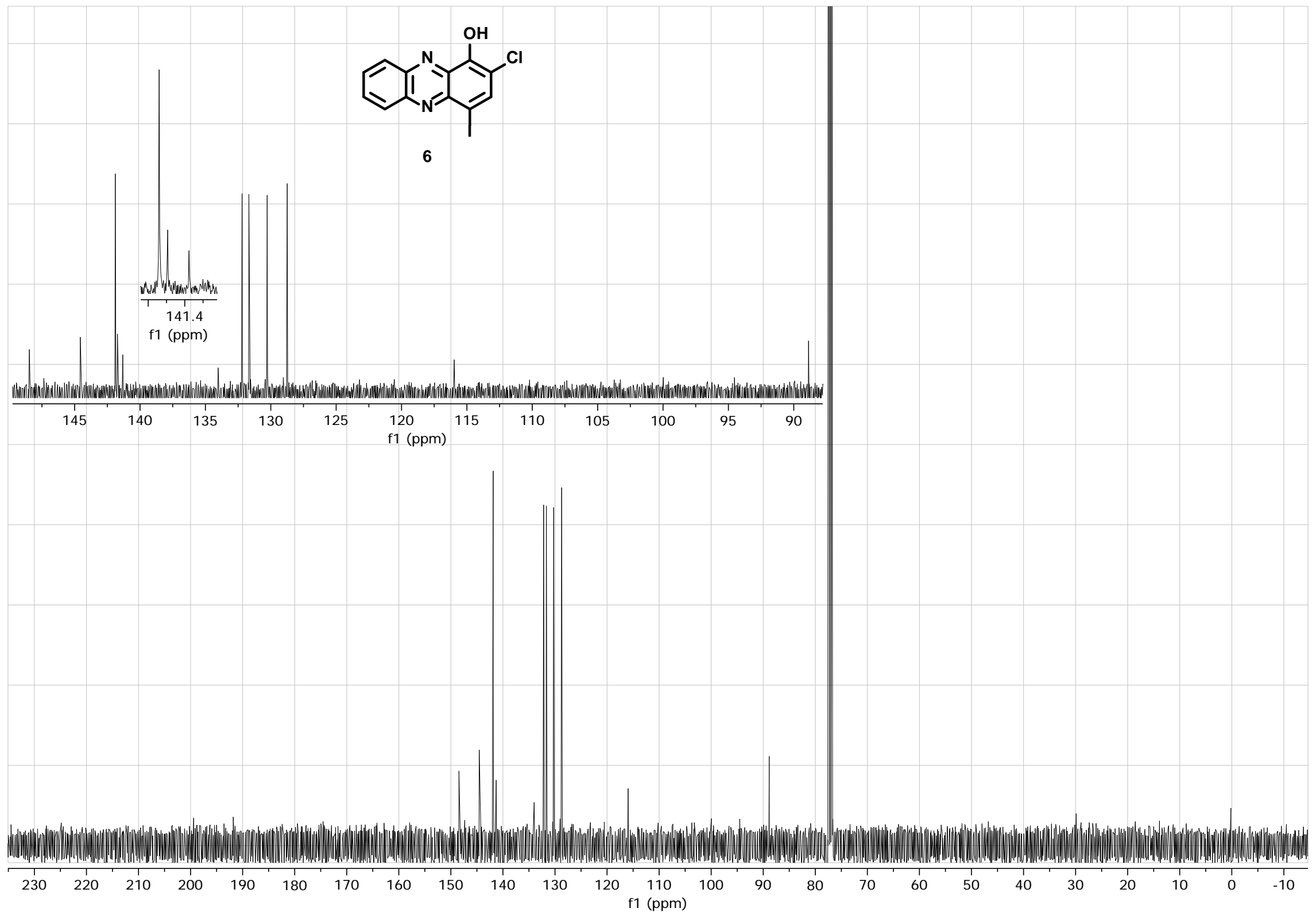




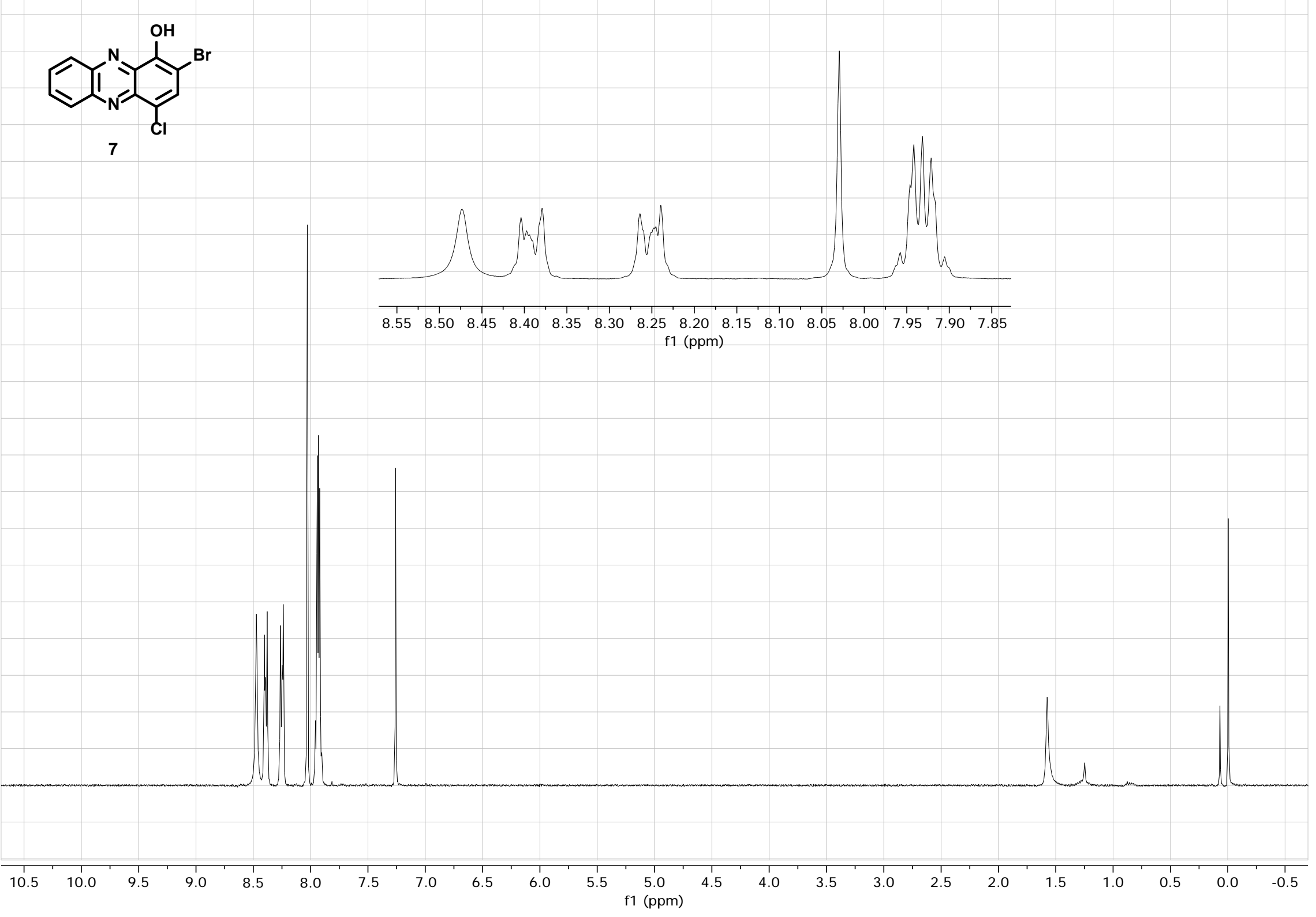




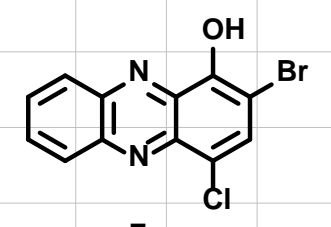

7
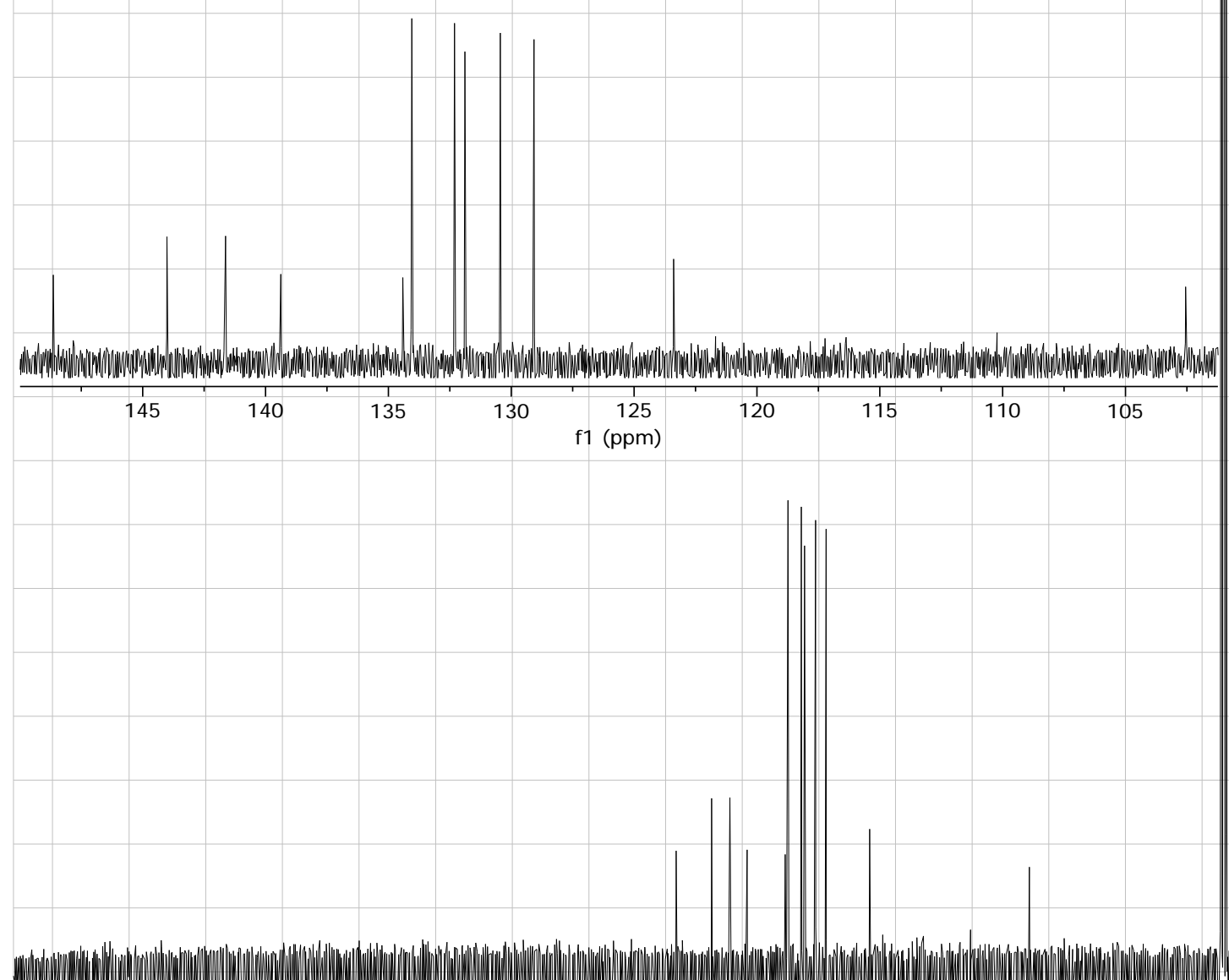

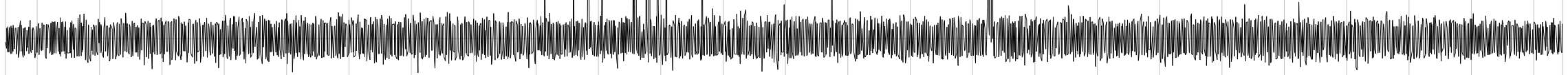

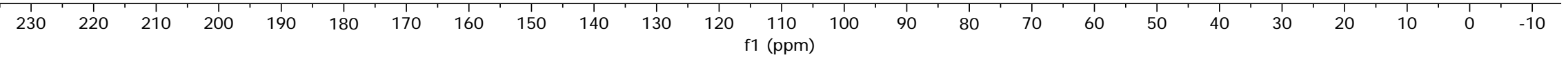




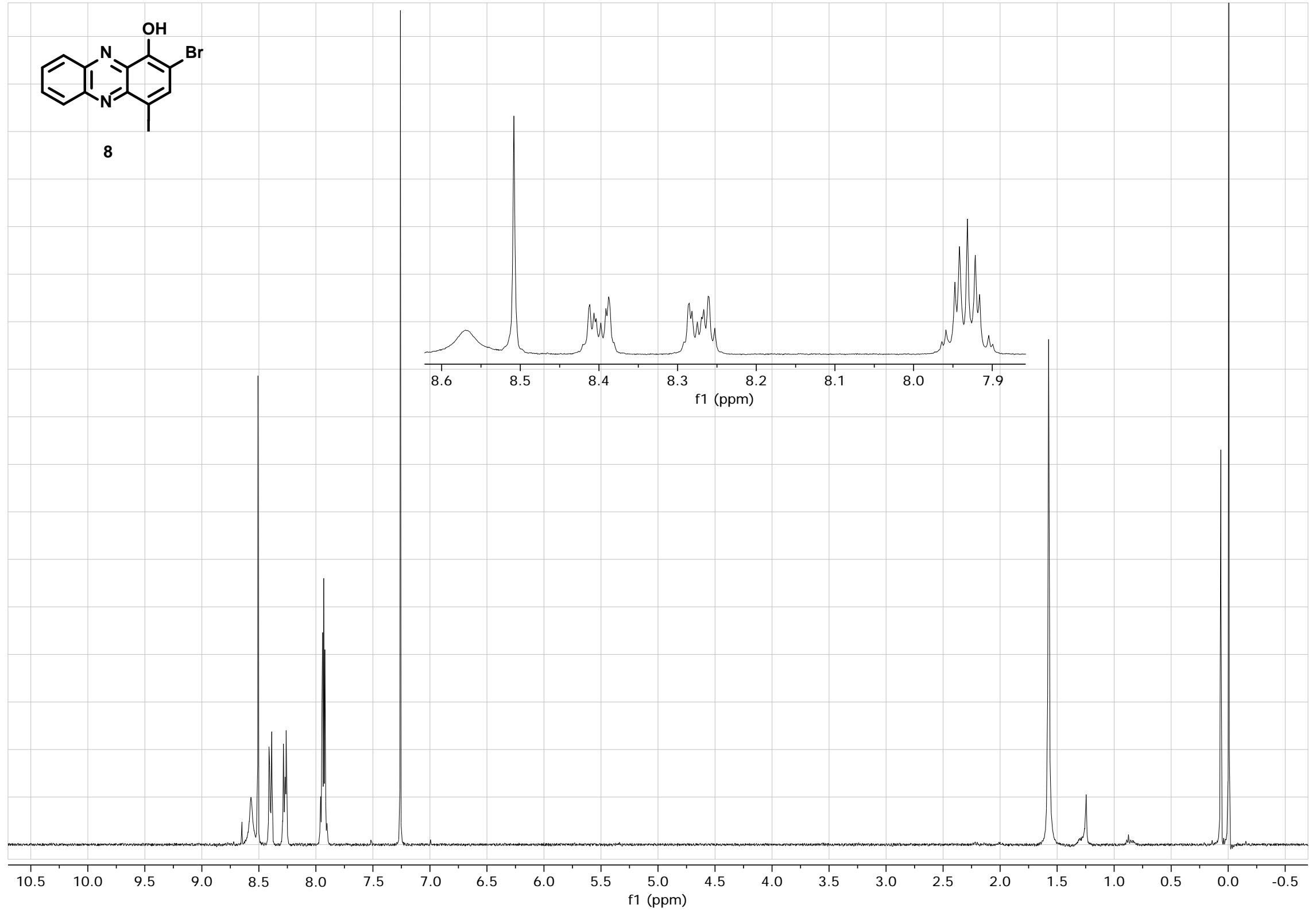

S53 


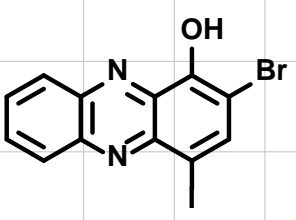

8
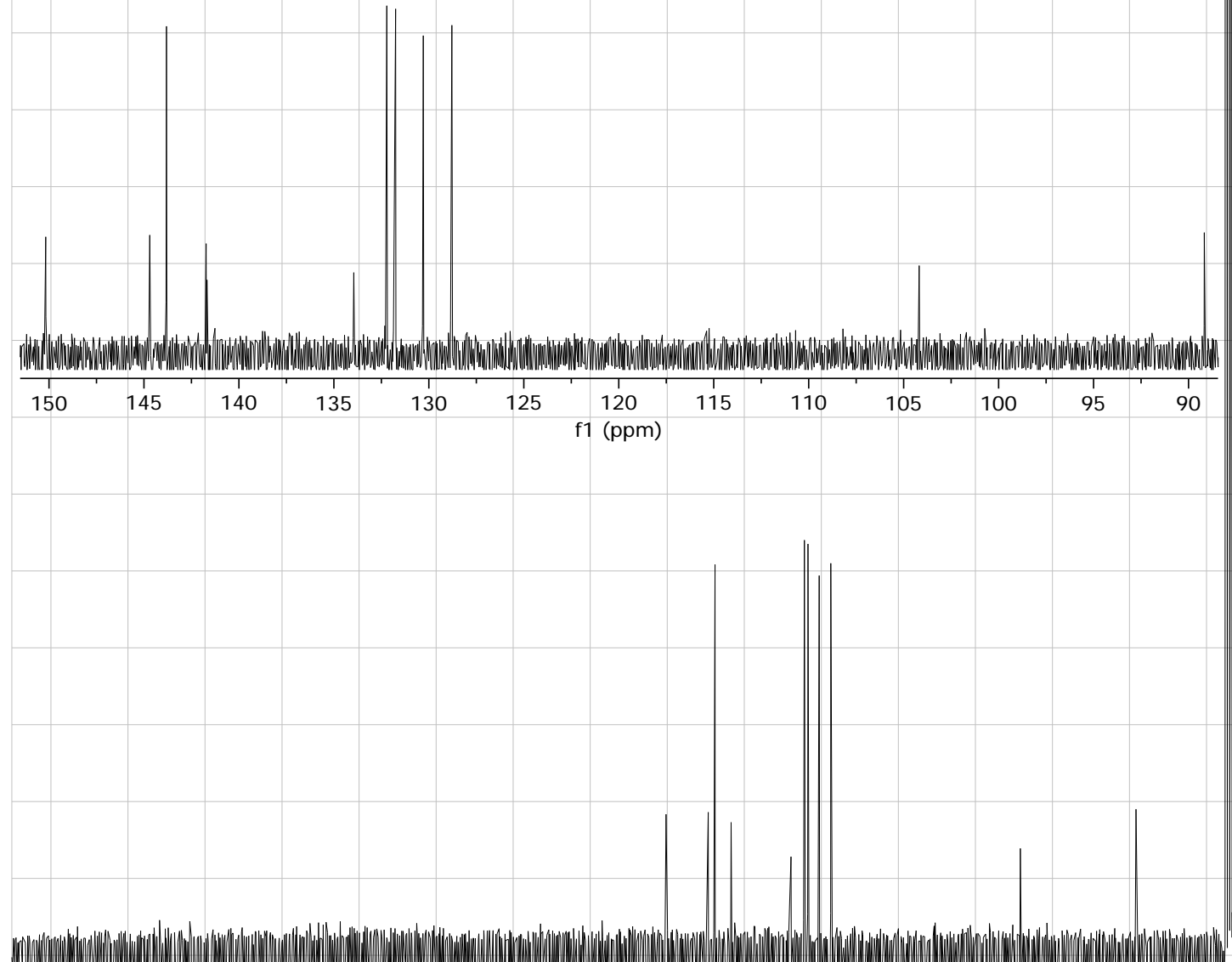

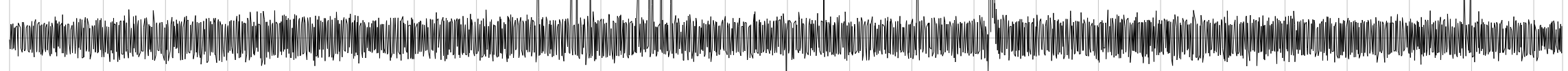

$\begin{array}{lllllllllllllllllllllllllll}230 & 220 & 210 & 200 & 190 & 180 & 170 & 160 & 150 & 140 & 130 & 120 & \begin{array}{c}110 \\ \mathrm{f} 1(\mathrm{ppm})\end{array} & 100 & 90 & 80 & 70 & 60 & 50 & 40 & 30 & 20 & 10 & 0 & -10\end{array}$




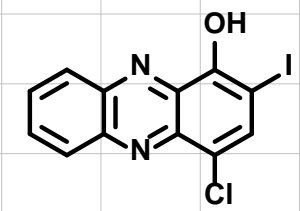

9

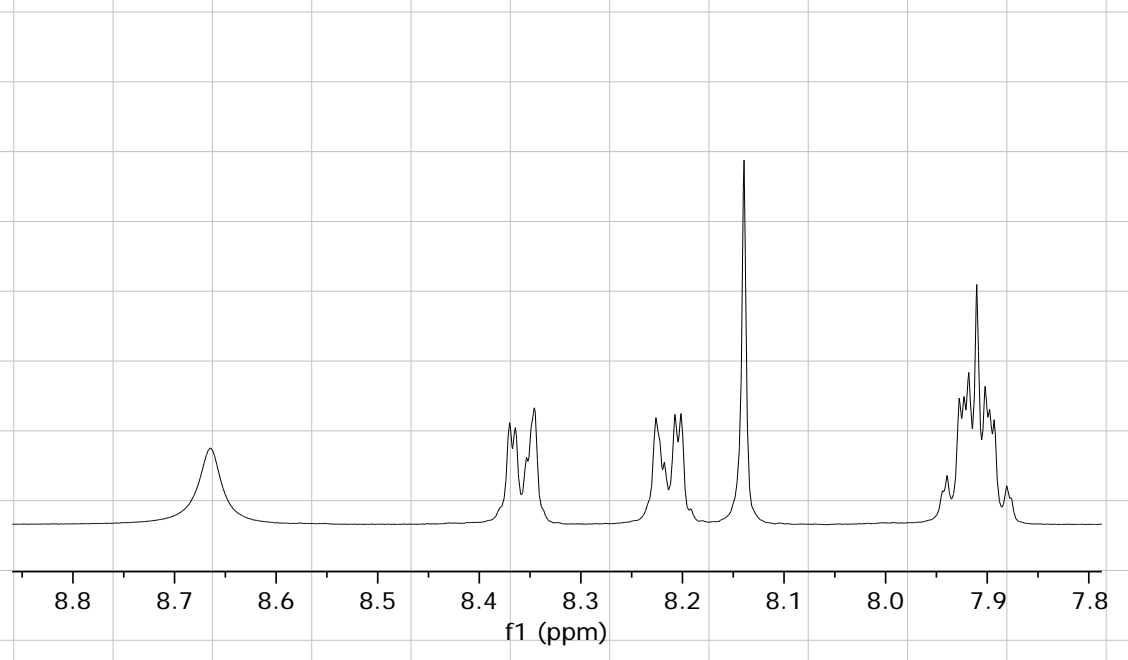




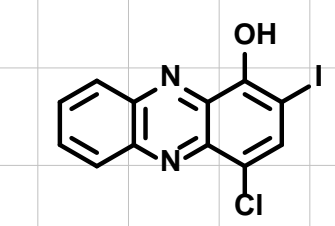

9

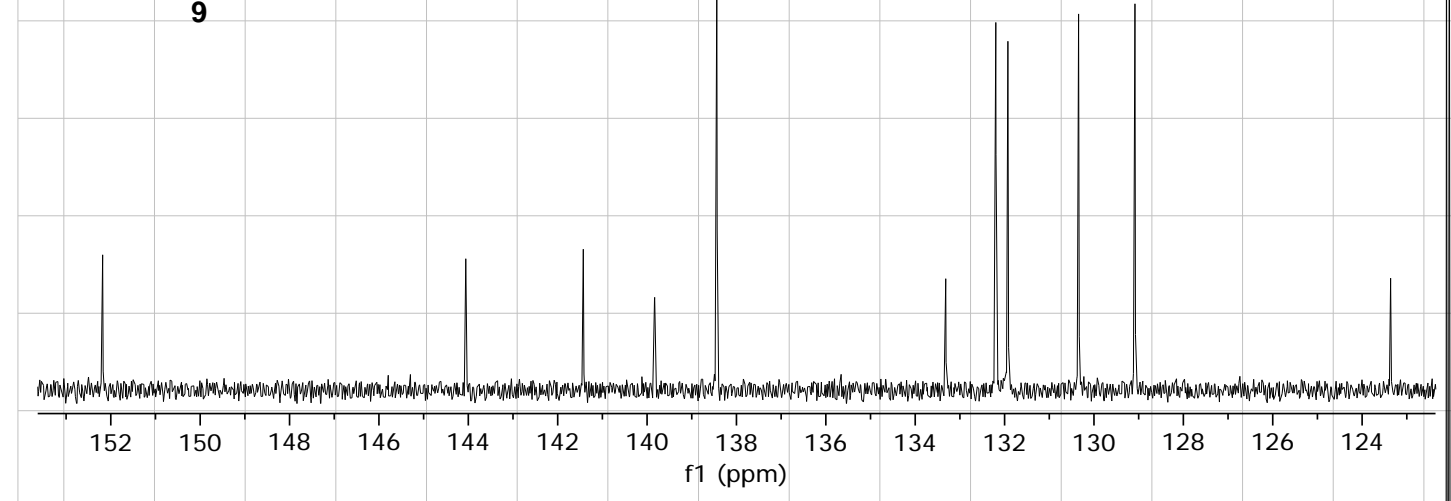

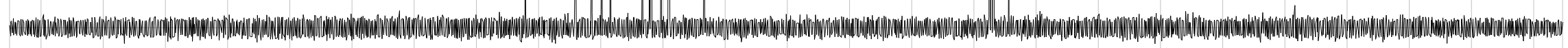

$\begin{array}{llllllllllllllllllllllllllll}230 & 220 & 210 & 200 & 190 & 180 & 170 & 160 & 150 & 140 & 130 & 120 & \begin{array}{c}110 \\ \mathrm{f} 1(\mathrm{ppm})\end{array} & 100 & 90 & 80 & 70 & 60 & 50 & 40 & 30 & 20 & 10 & 0 & -10\end{array}$




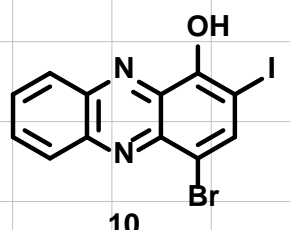




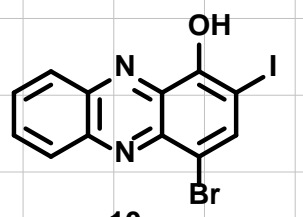

10

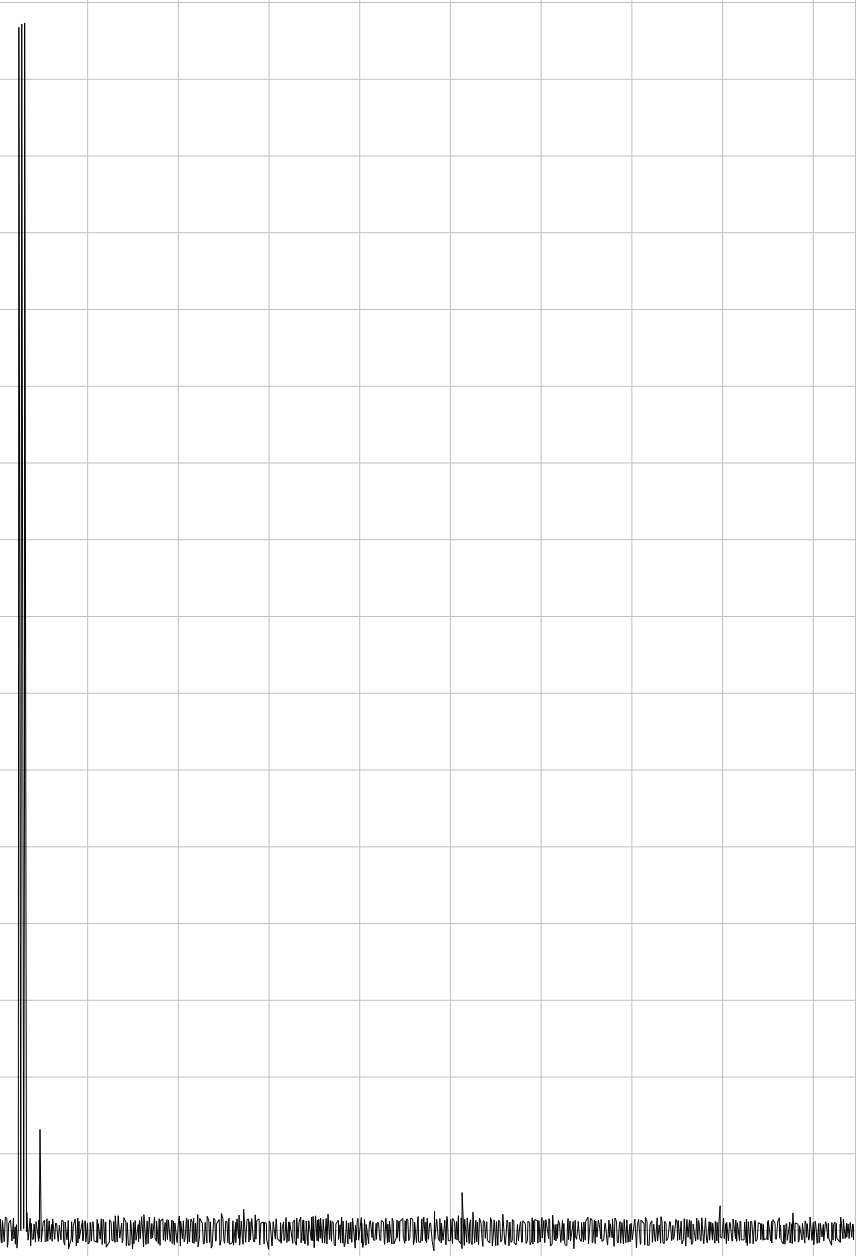

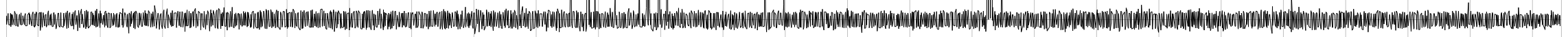




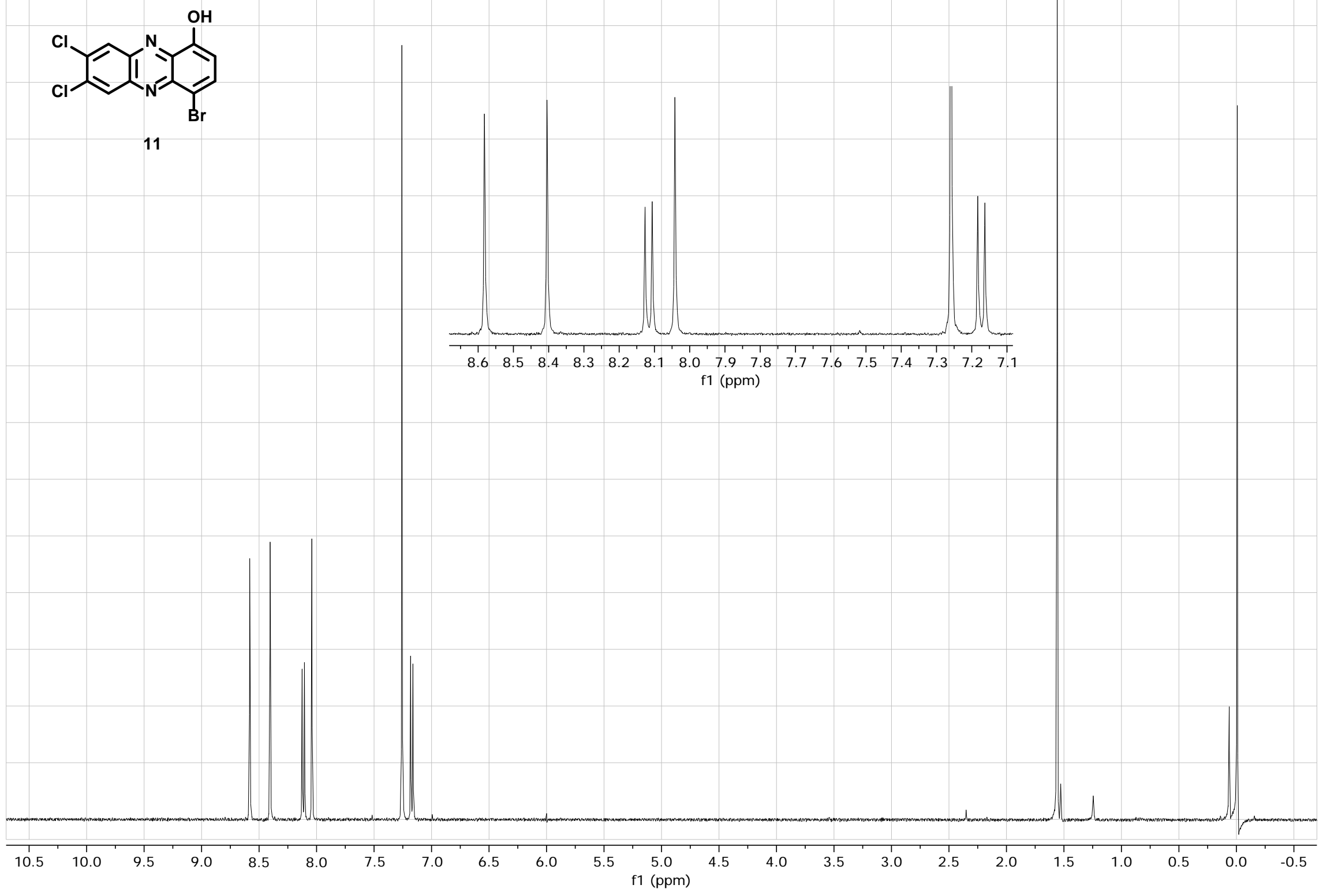




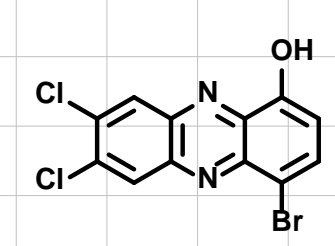

11

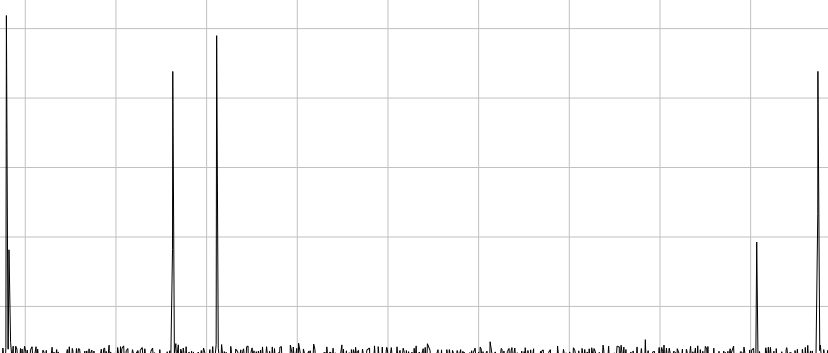

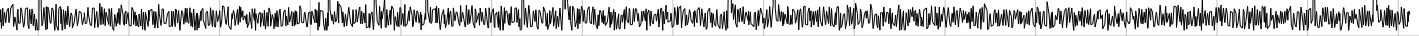

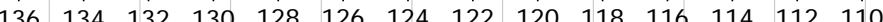
$\mathrm{fl}(\mathrm{ppm})$

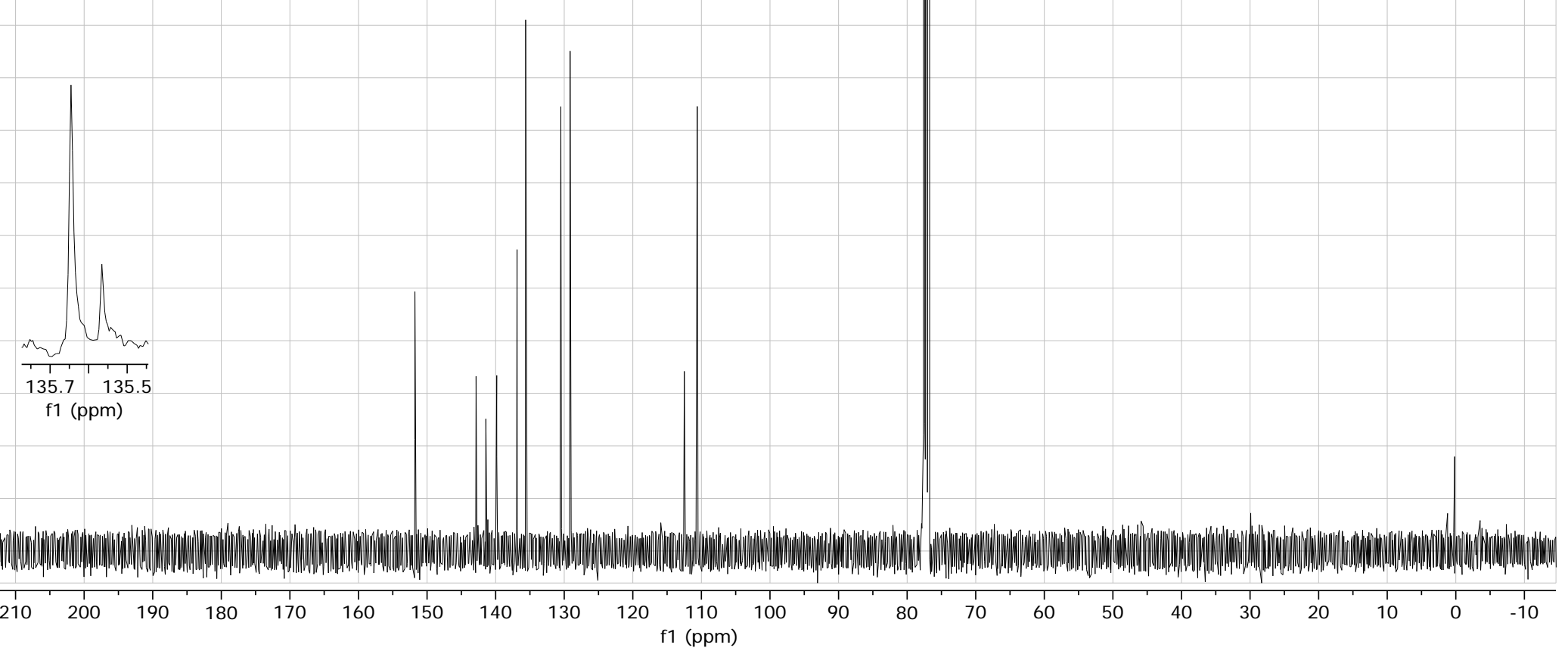




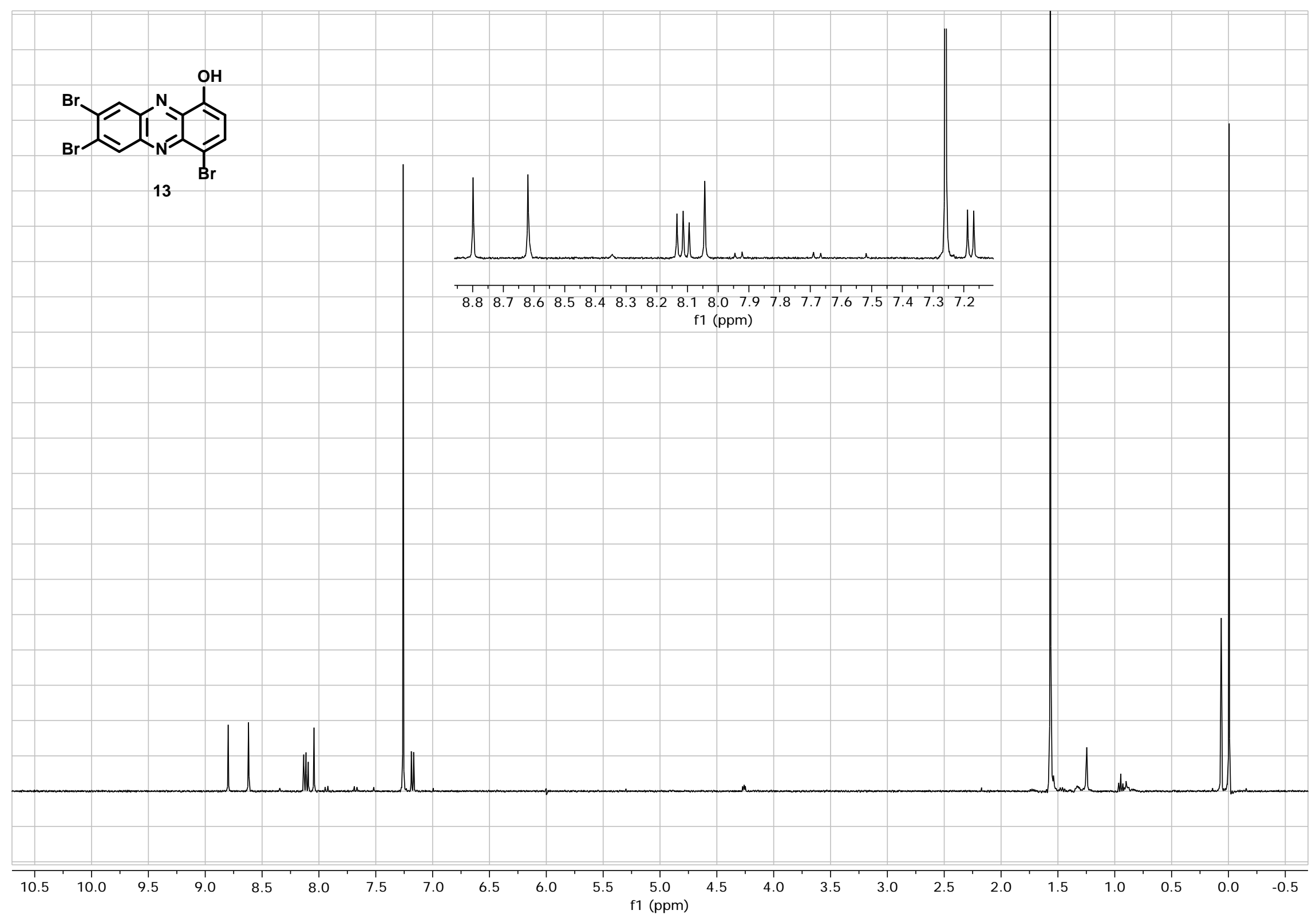




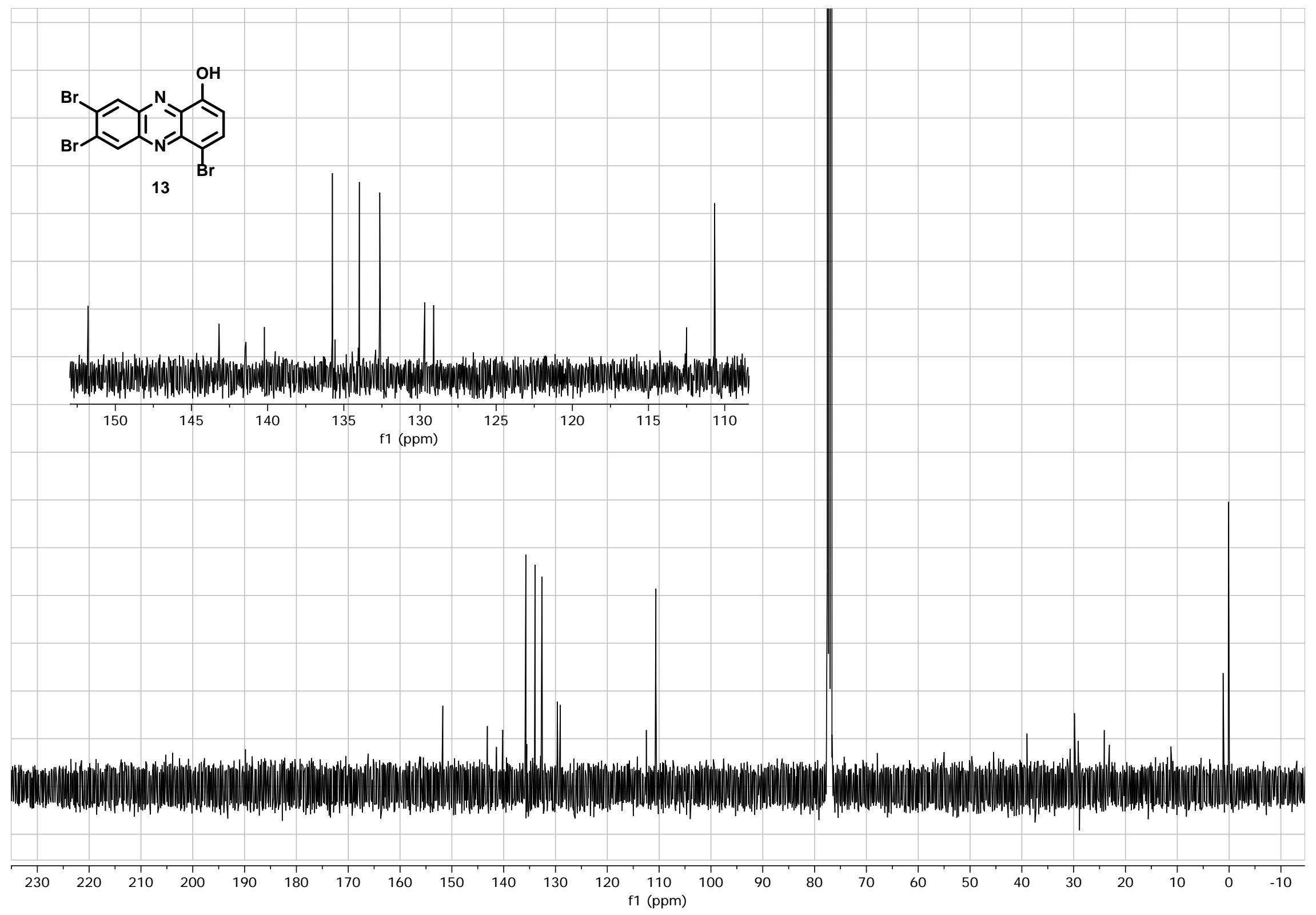




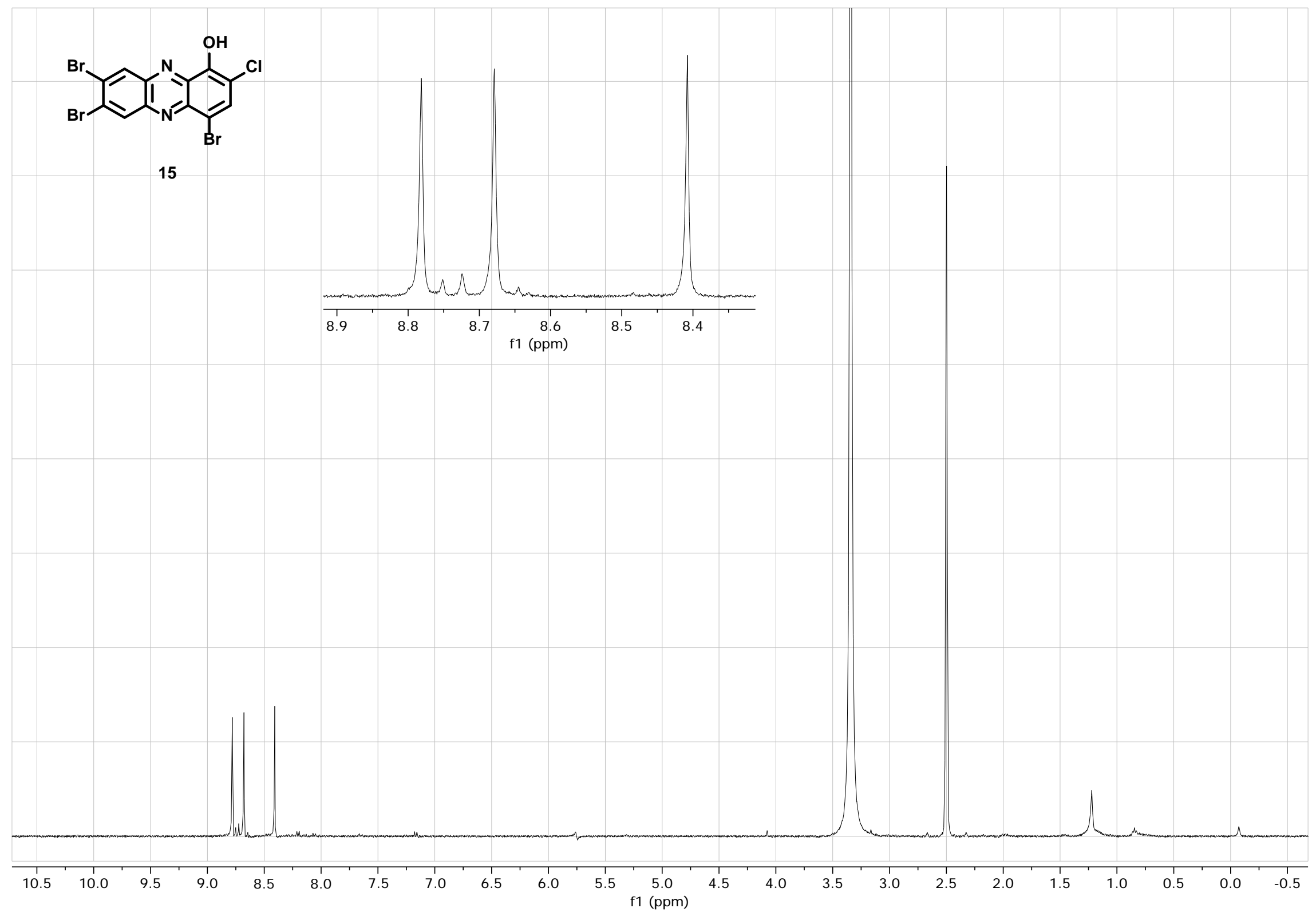

S63 


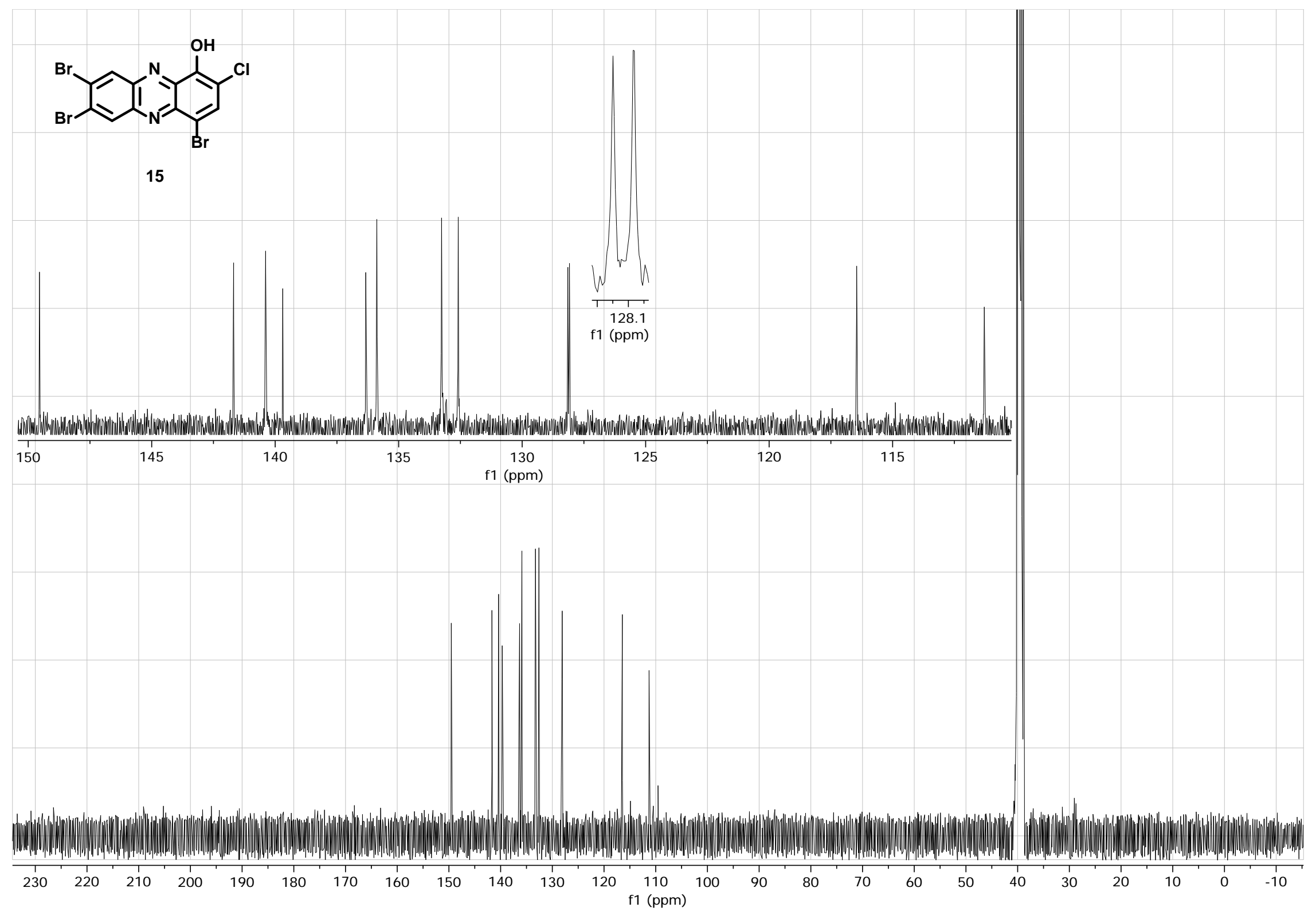




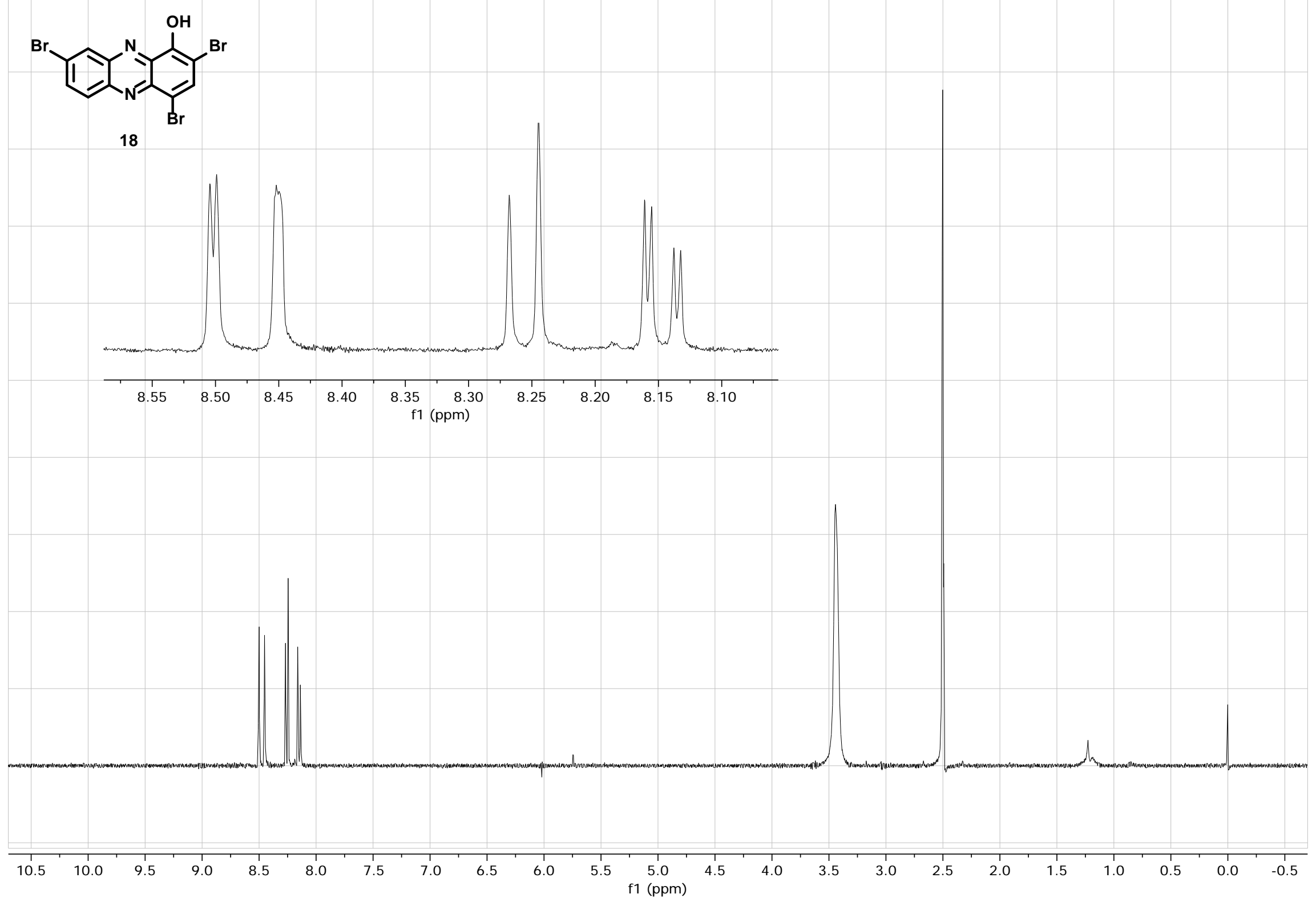




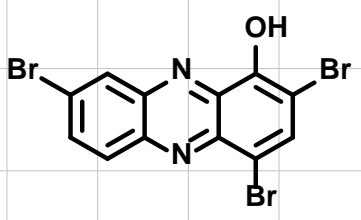

18

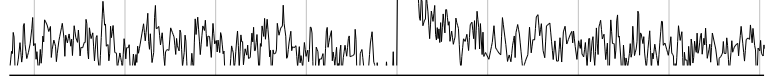

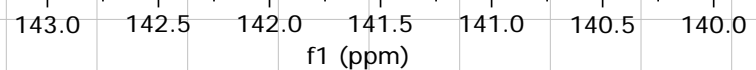

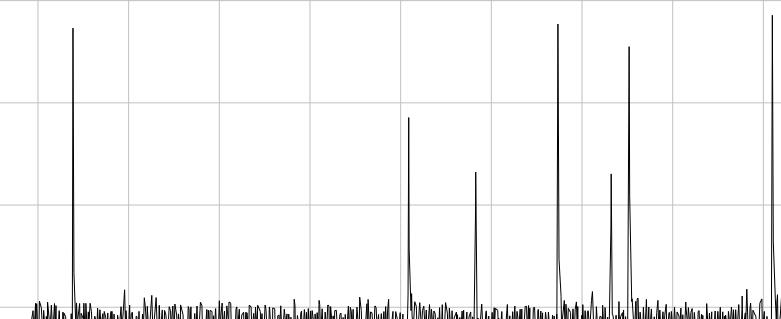

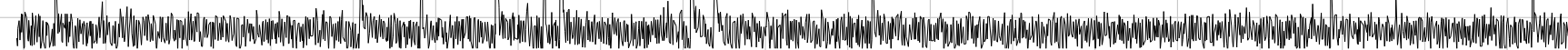

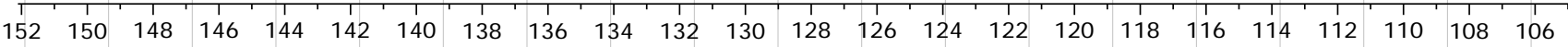
$\mathrm{f1}(\mathrm{ppm})$

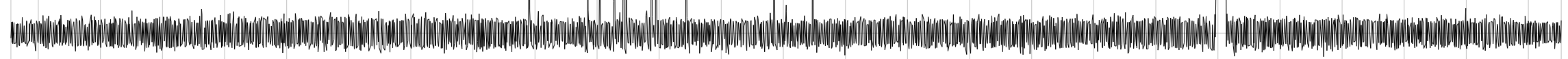

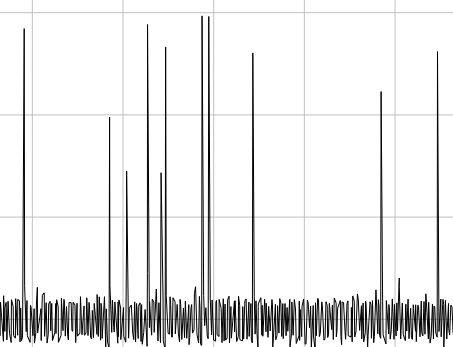

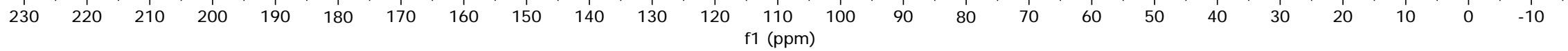




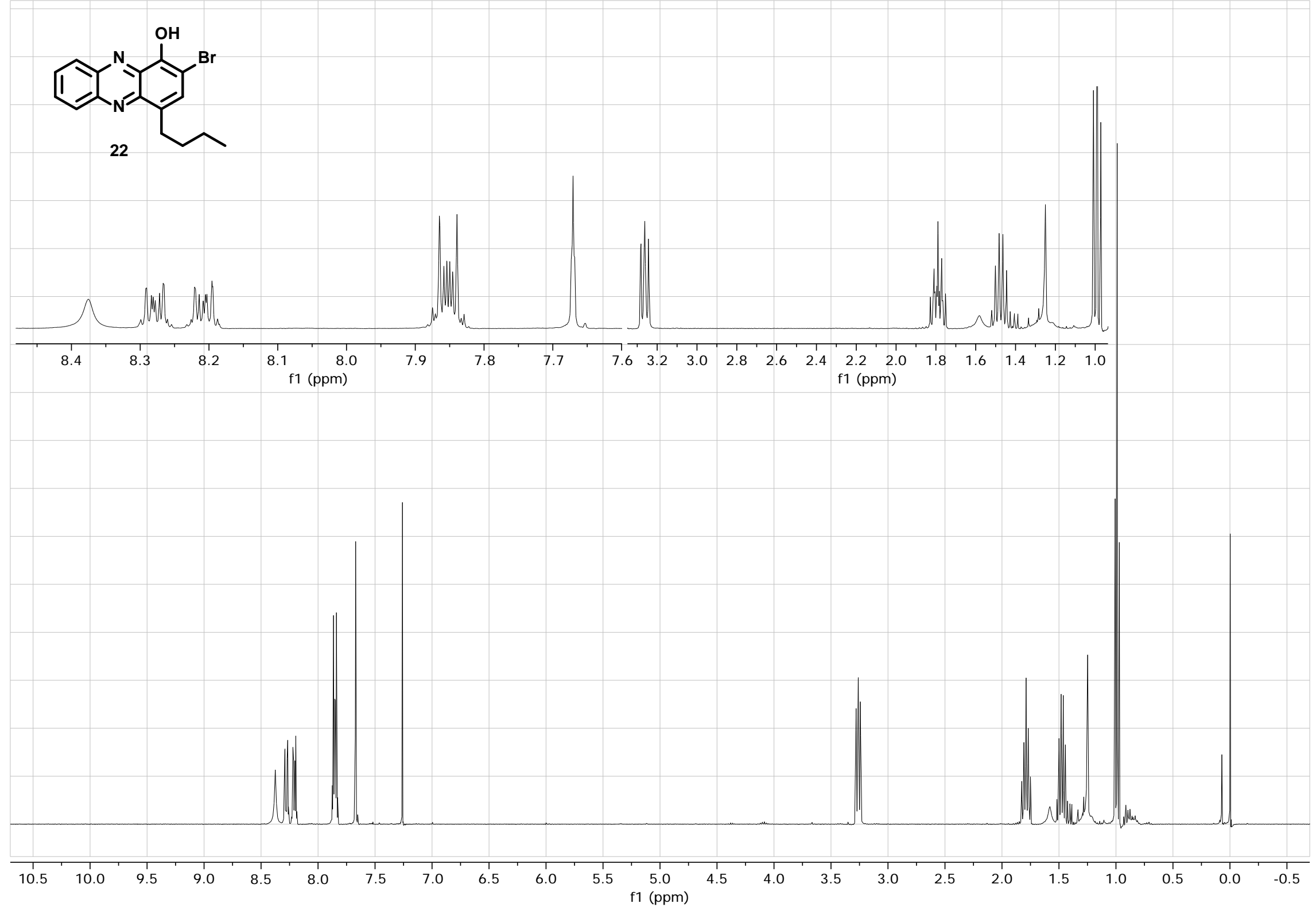




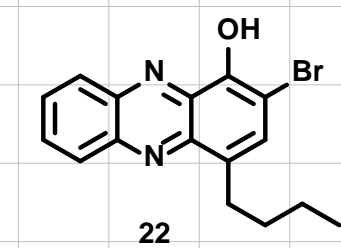

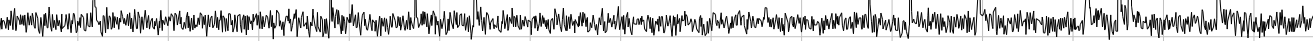

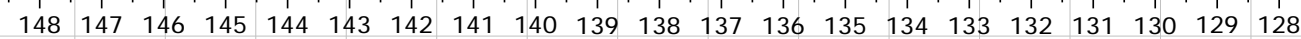
f1 (ppm)

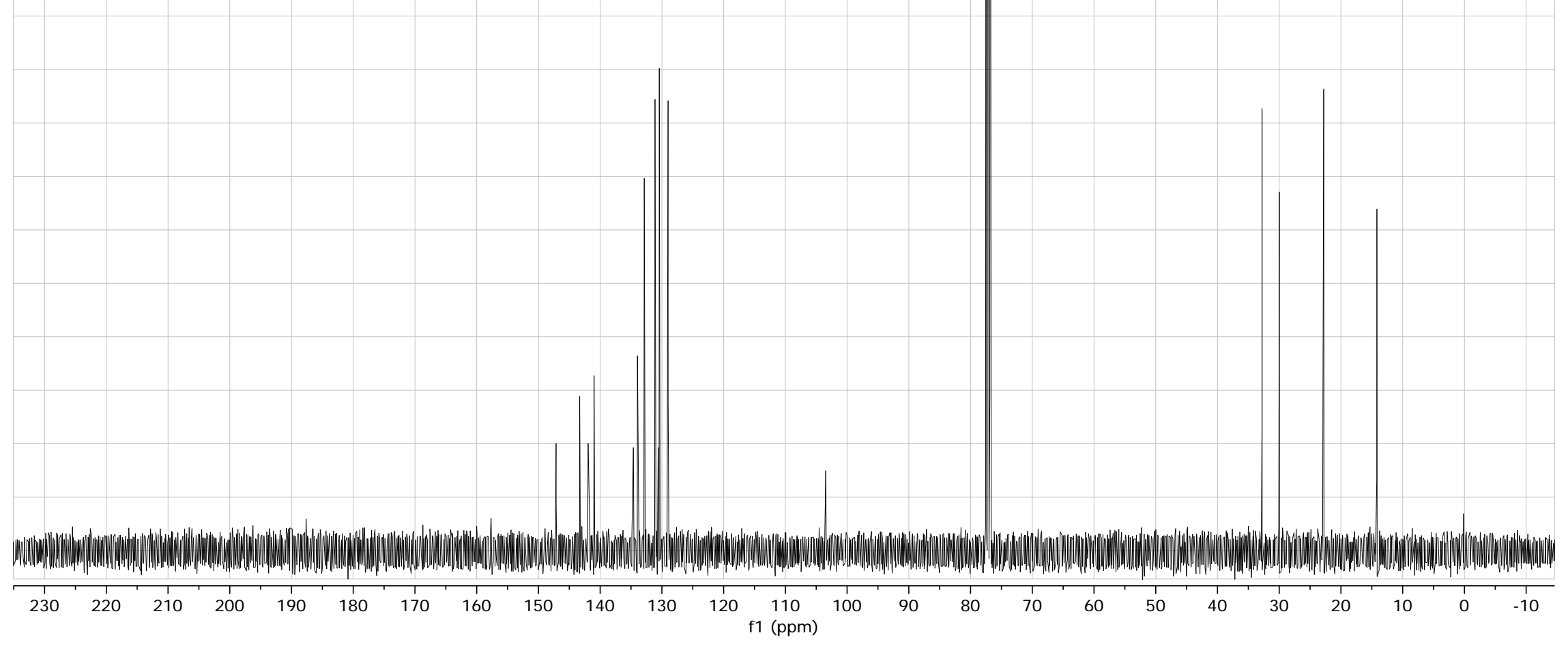




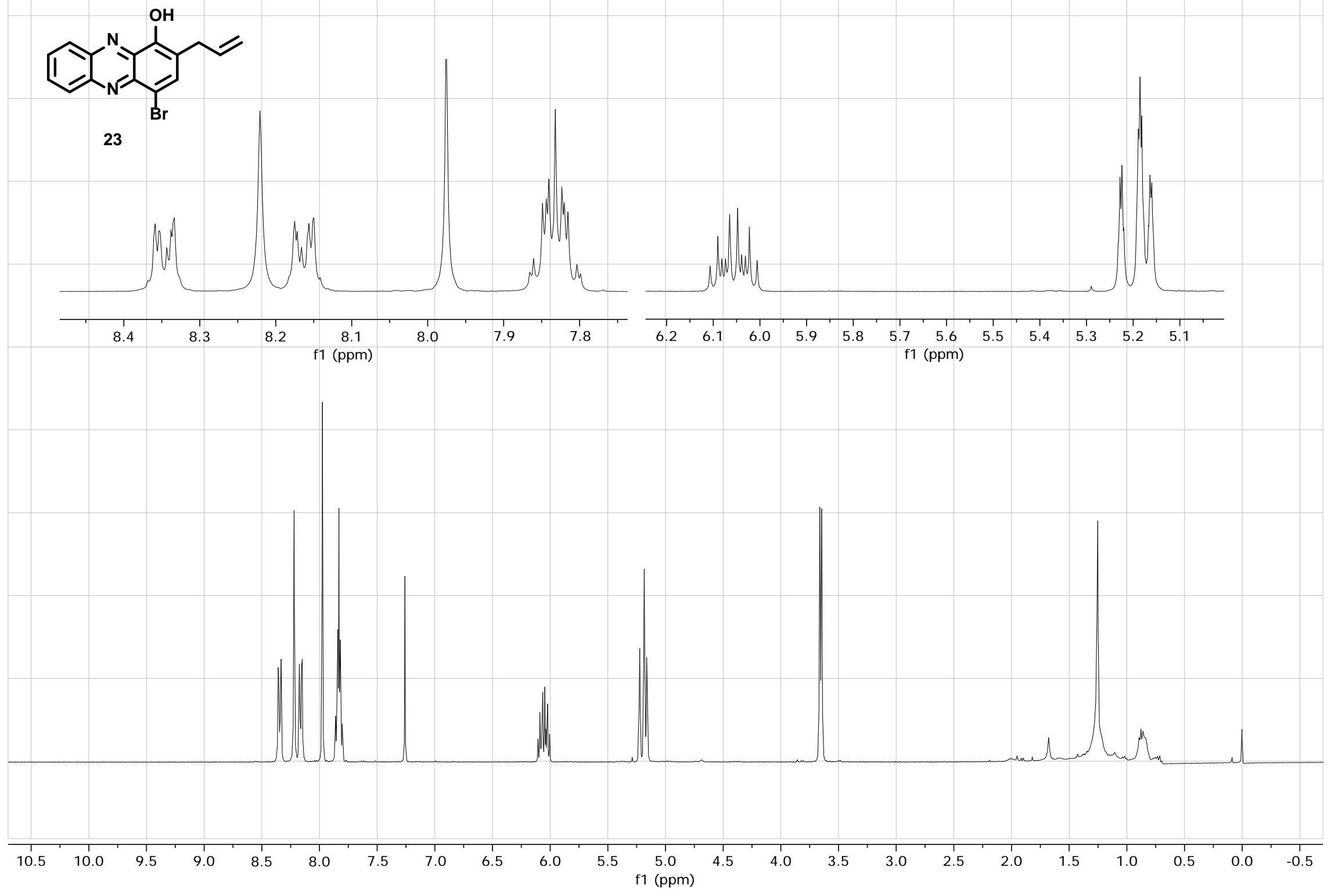




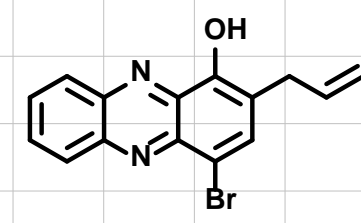

23

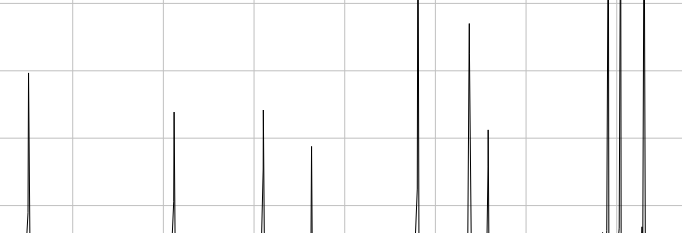

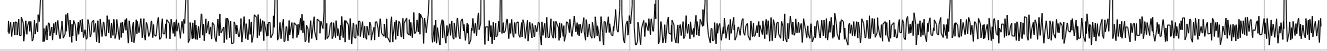

$\begin{array}{llllllllllllllllllll}148 & 146 & 144 & 142 & 140 & 138 & 136 & 134 & 132 & 130 & 128 & 126 & 124 & 122 & 120 & 118 & 116 & 114 & 112\end{array}$ f1 (ppm)

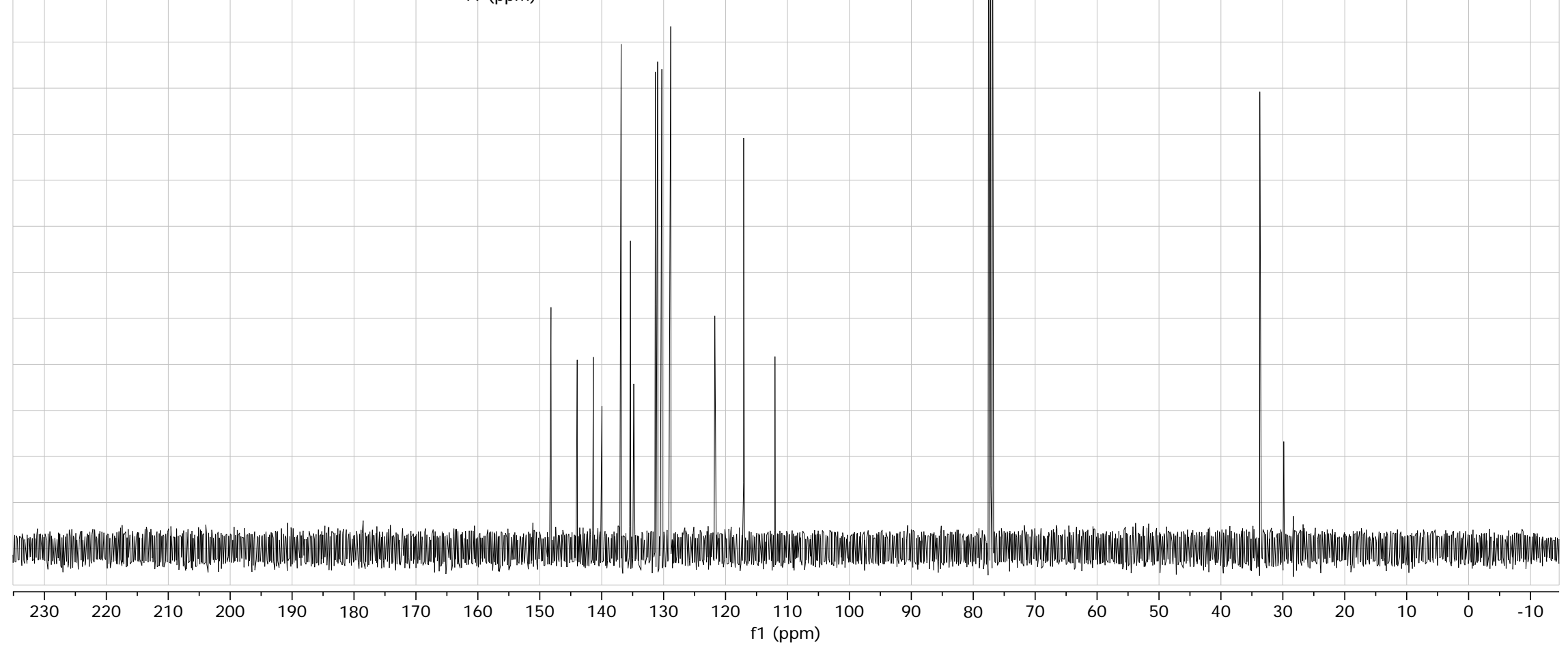




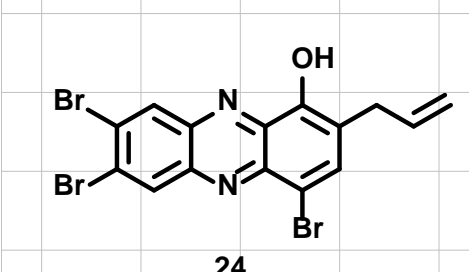

24

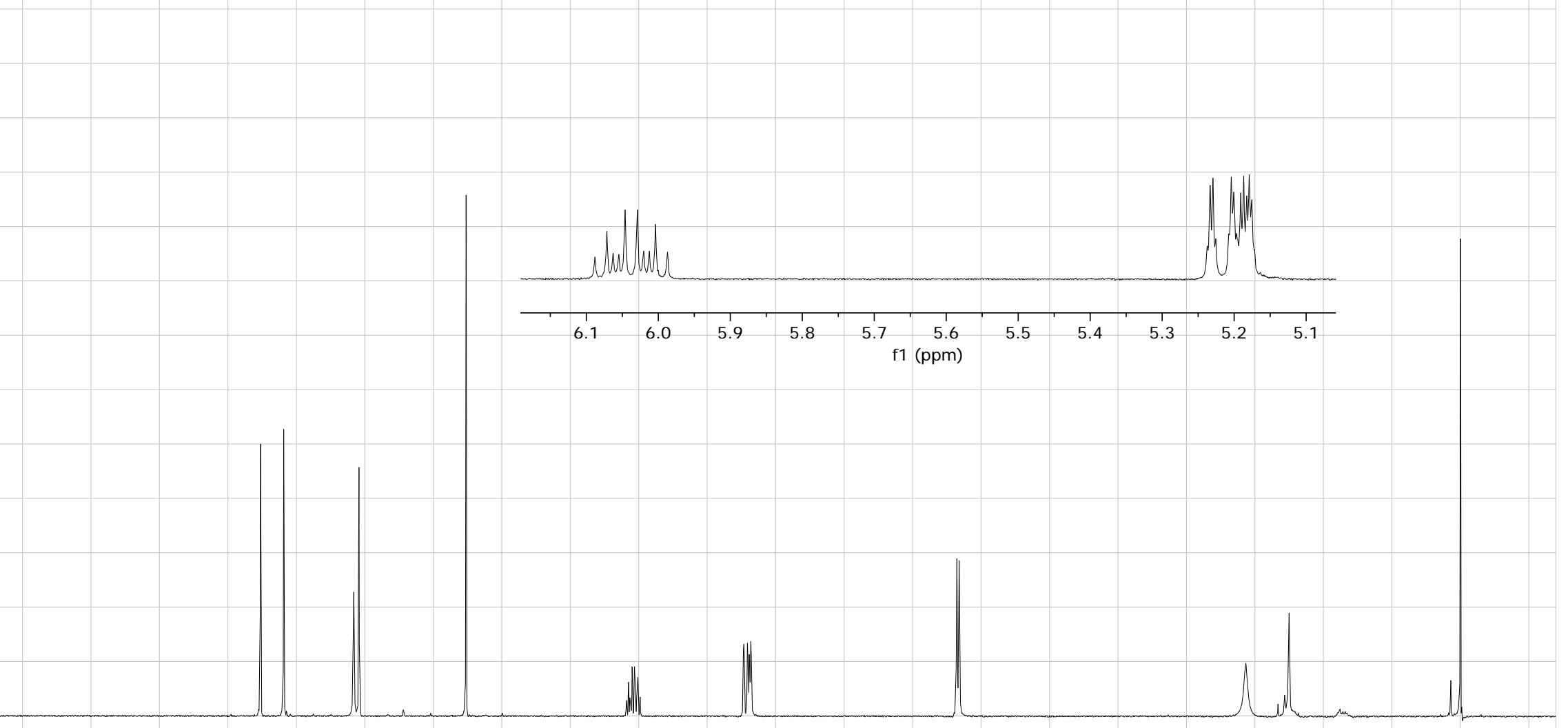




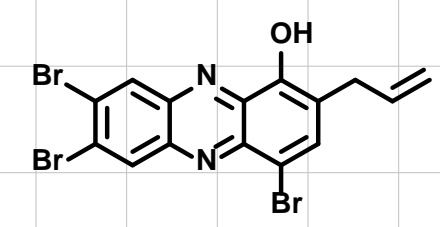

24

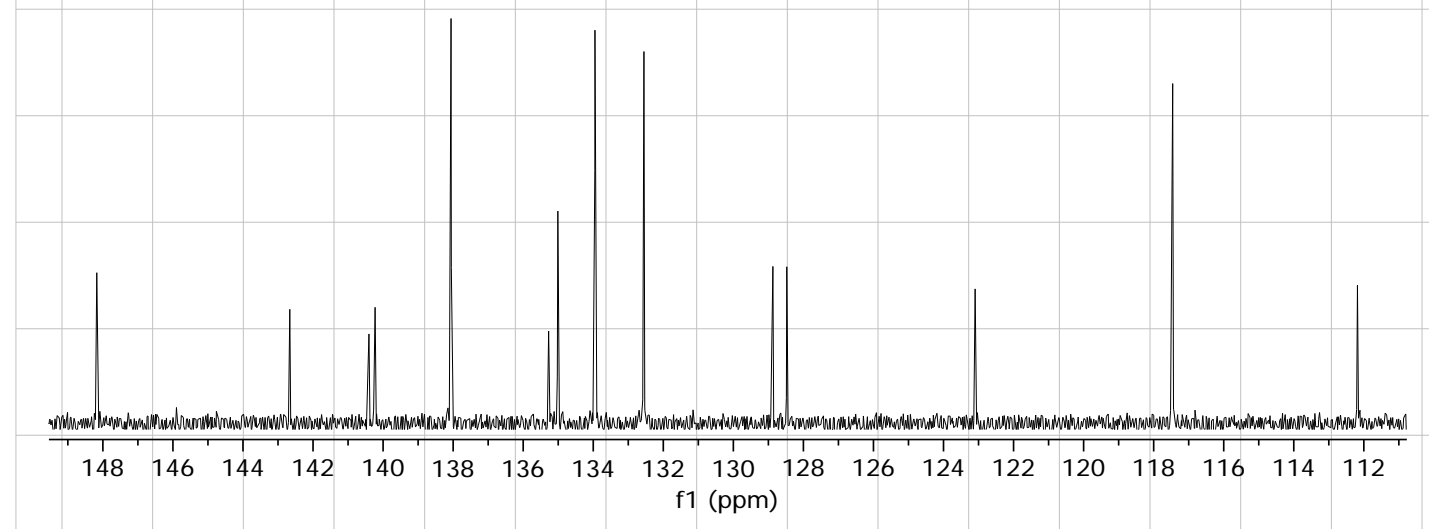

rim.

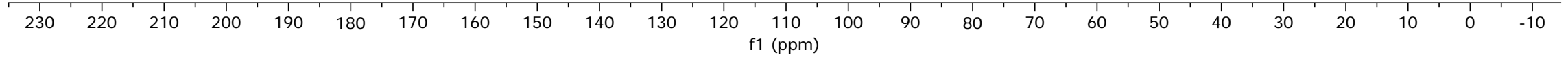




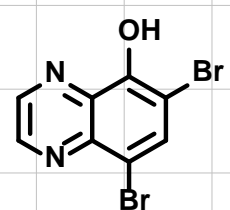

25

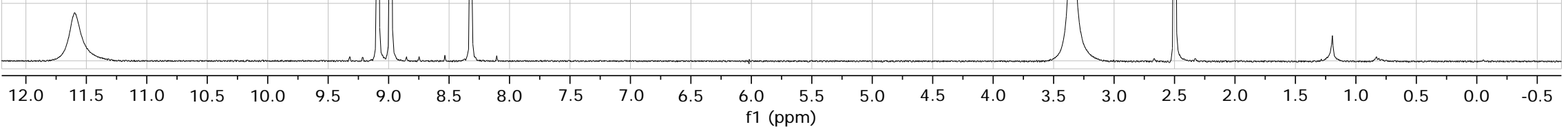

S73 


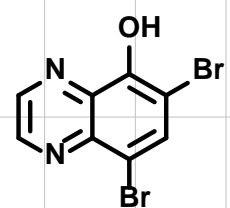

25

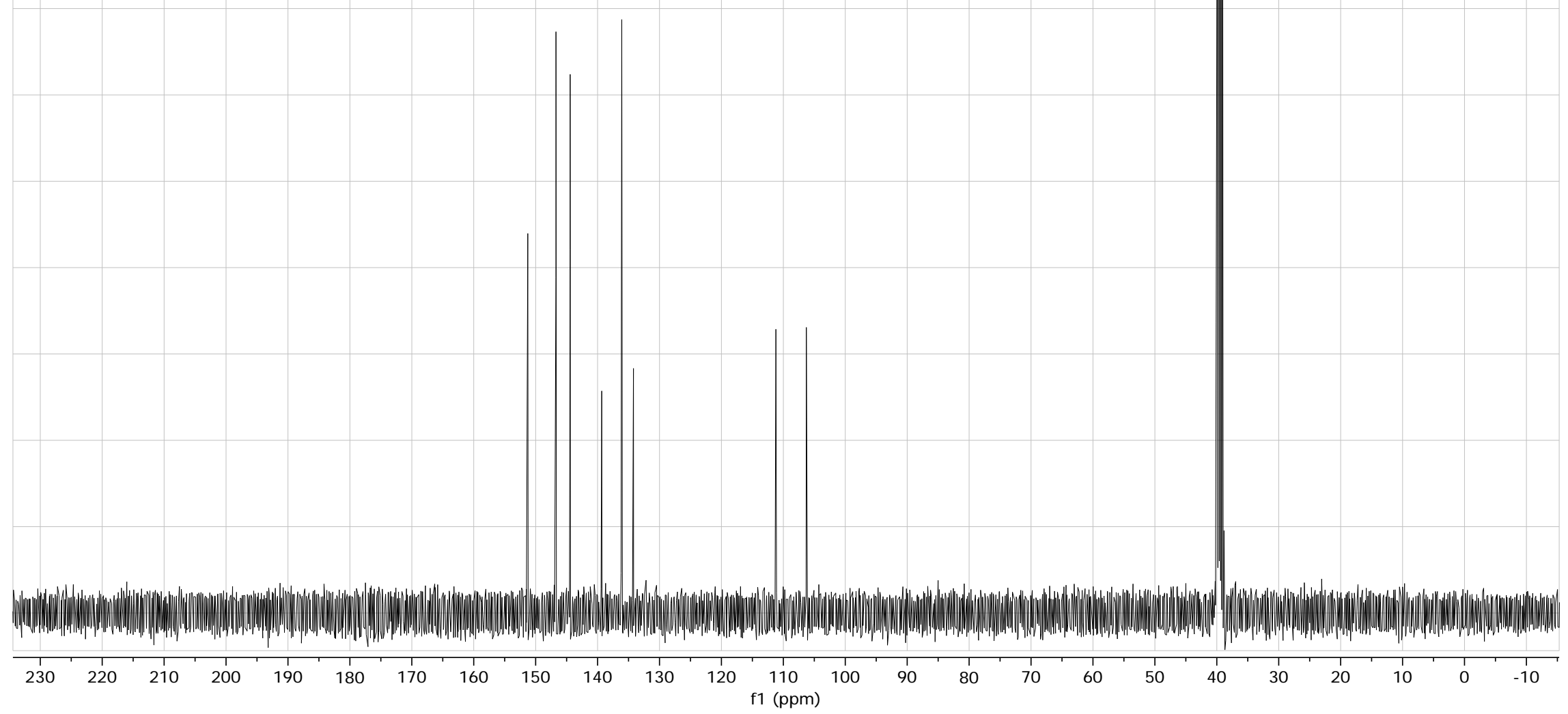

S74 


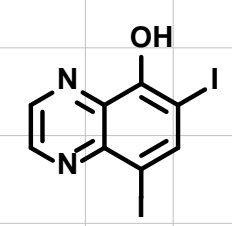

26

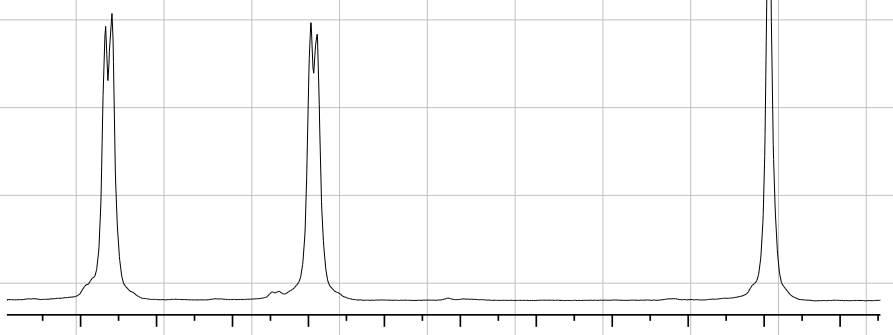

\begin{tabular}{lllll|llllll}
9.05 & 9.00 & 8.95 & 8.90 & 8.85 & 8.80 & 8.75 & 8.70 & 8.65 & 8.60 & 8.55
\end{tabular}

f1 (ppm)

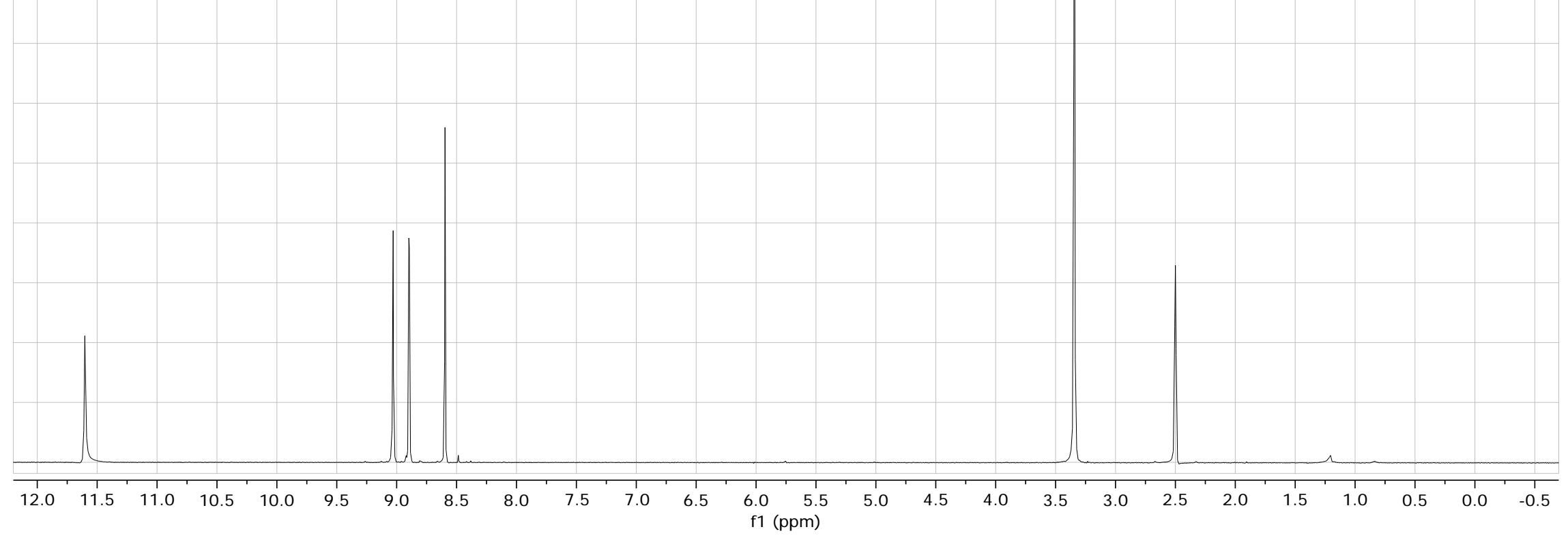




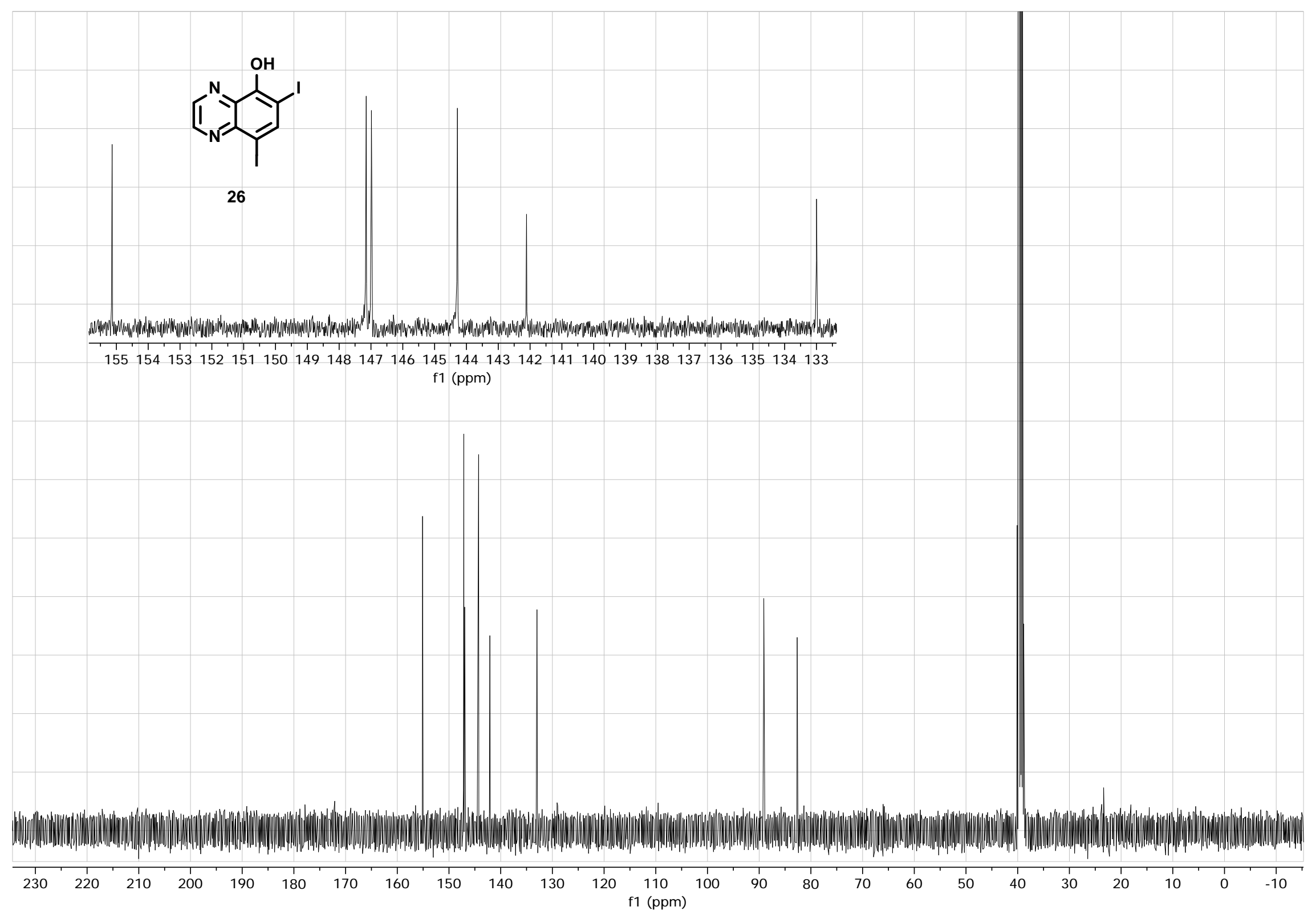




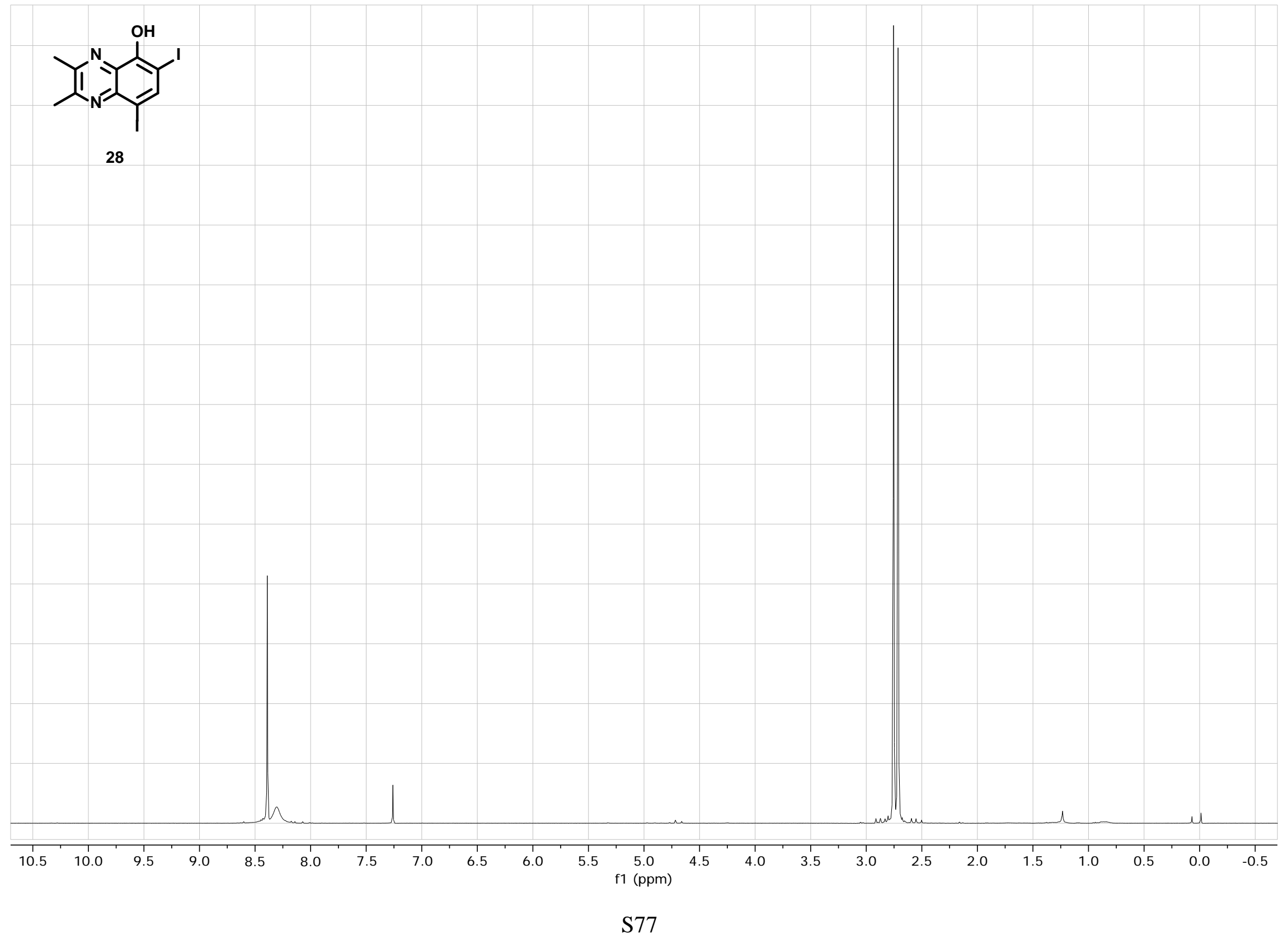




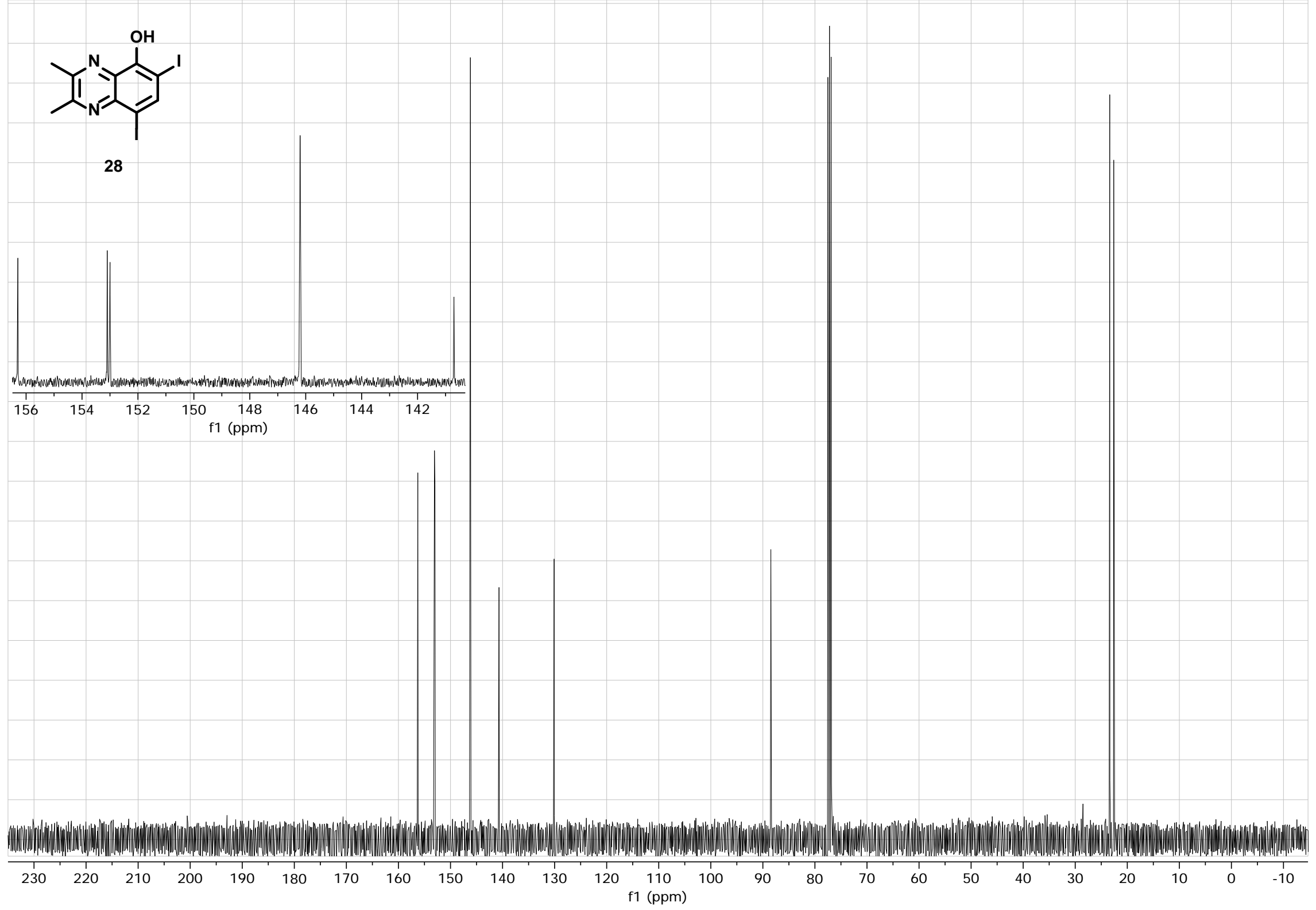

S78 


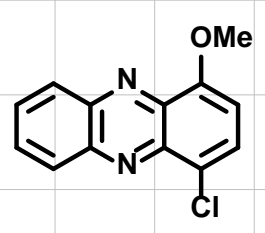

33

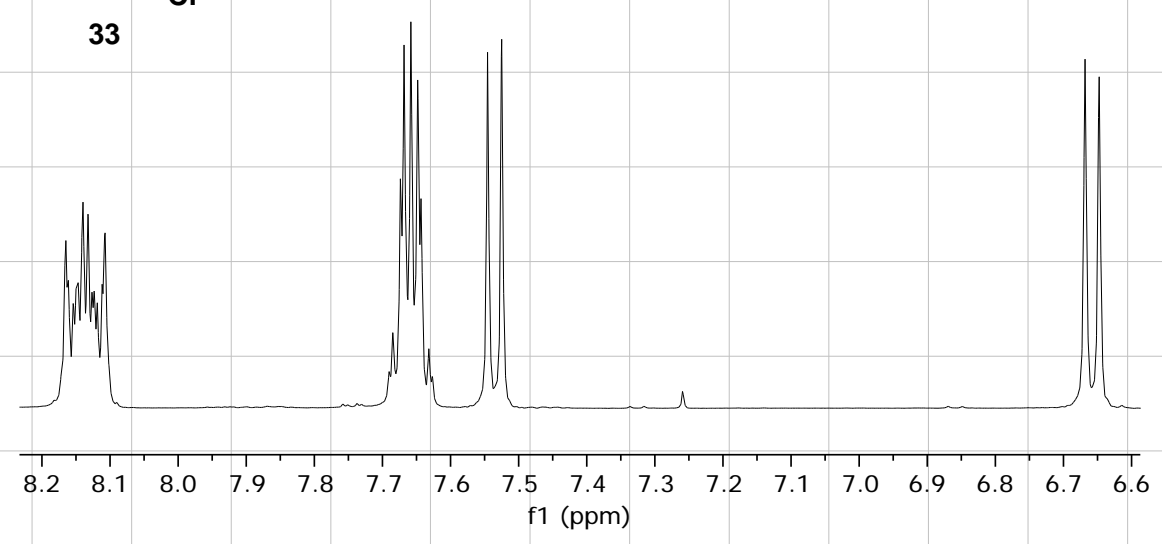

\begin{tabular}{|c|c|c|c|c|c|c|c|c|c|c|c|c|c|c|c|c|c|c|c|c|c|c|}
\hline 10.5 & 10.0 & 9.5 & 9.0 & 8.5 & 8.0 & 7.5 & 7.0 & 6.5 & 6.0 & 5.5 & $\begin{array}{c}5.0 \\
\text { f1 (ppm) }\end{array}$ & 4.5 & 4.0 & 3.5 & 3.0 & 2.5 & 2.0 & 1.5 & 1.0 & 0.5 & 0.0 & -0.5 \\
\hline
\end{tabular}



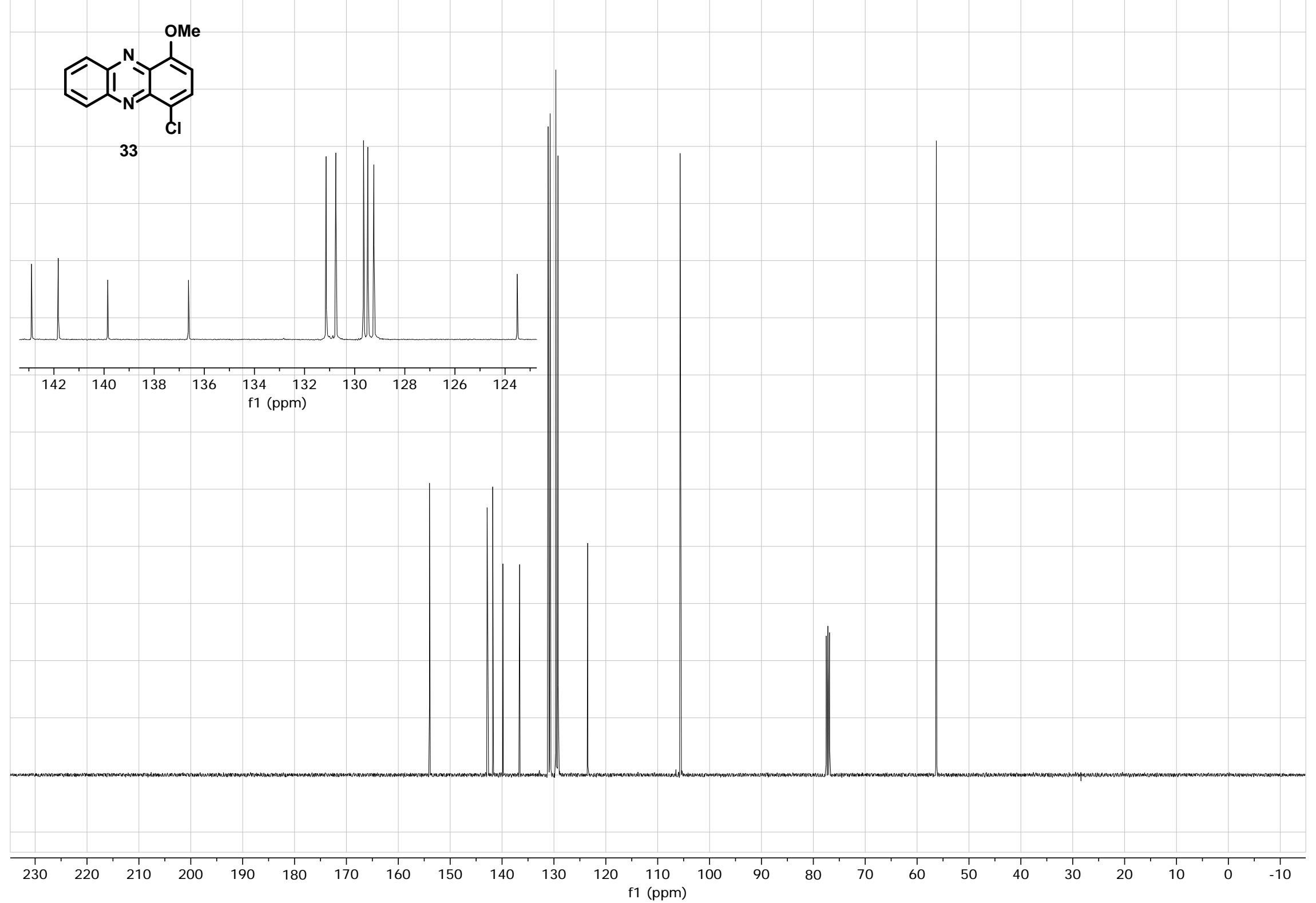


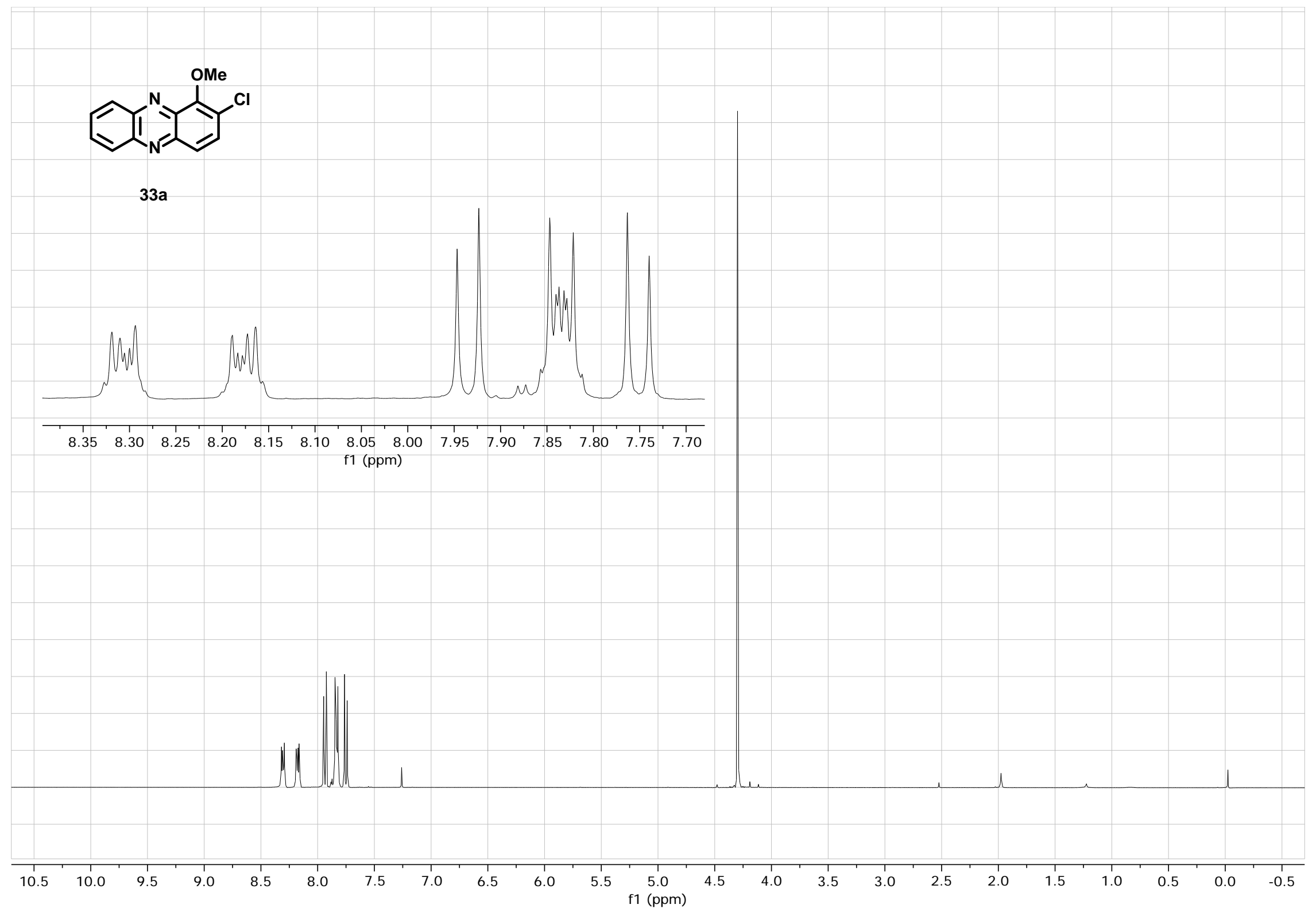




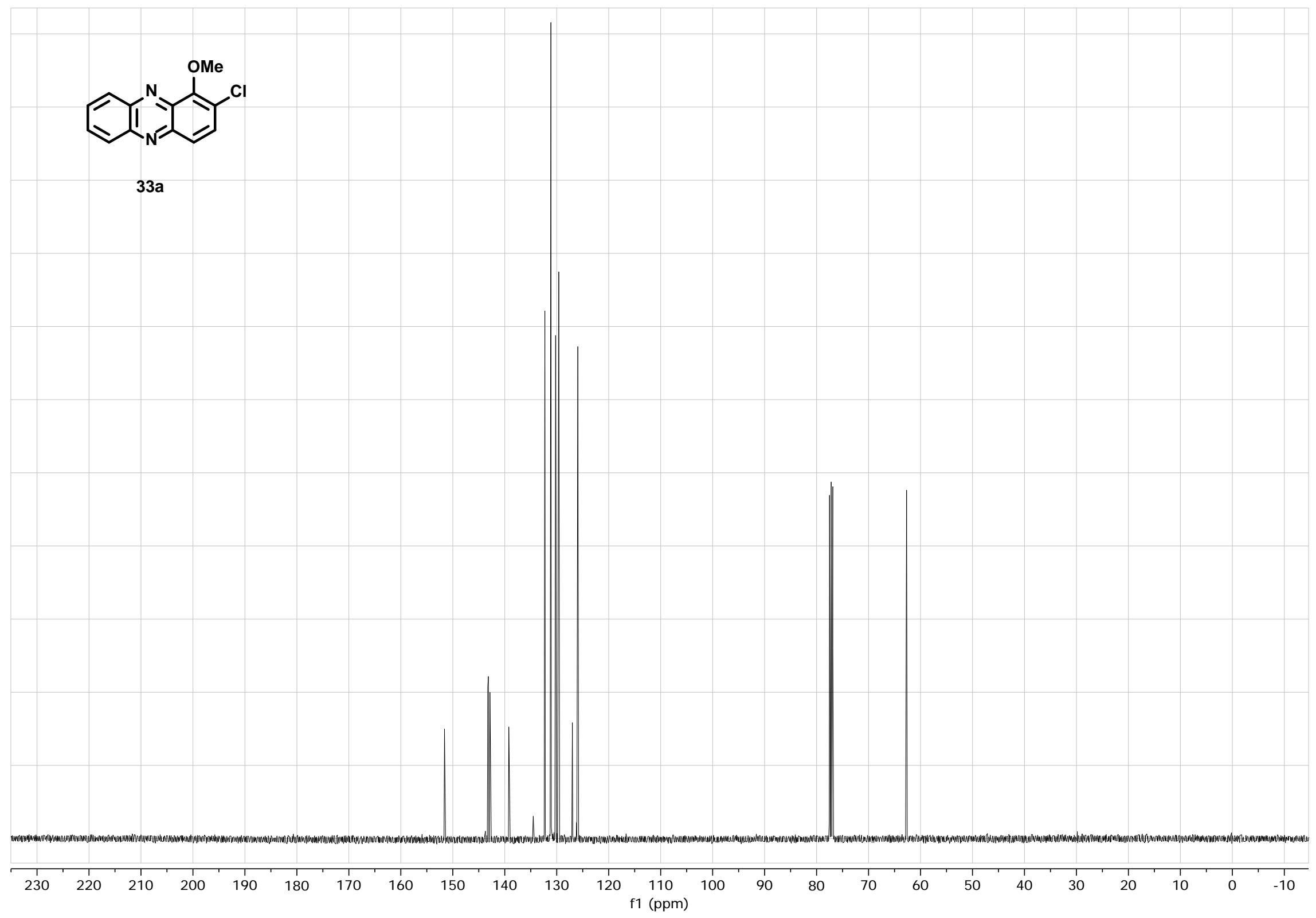

S82 


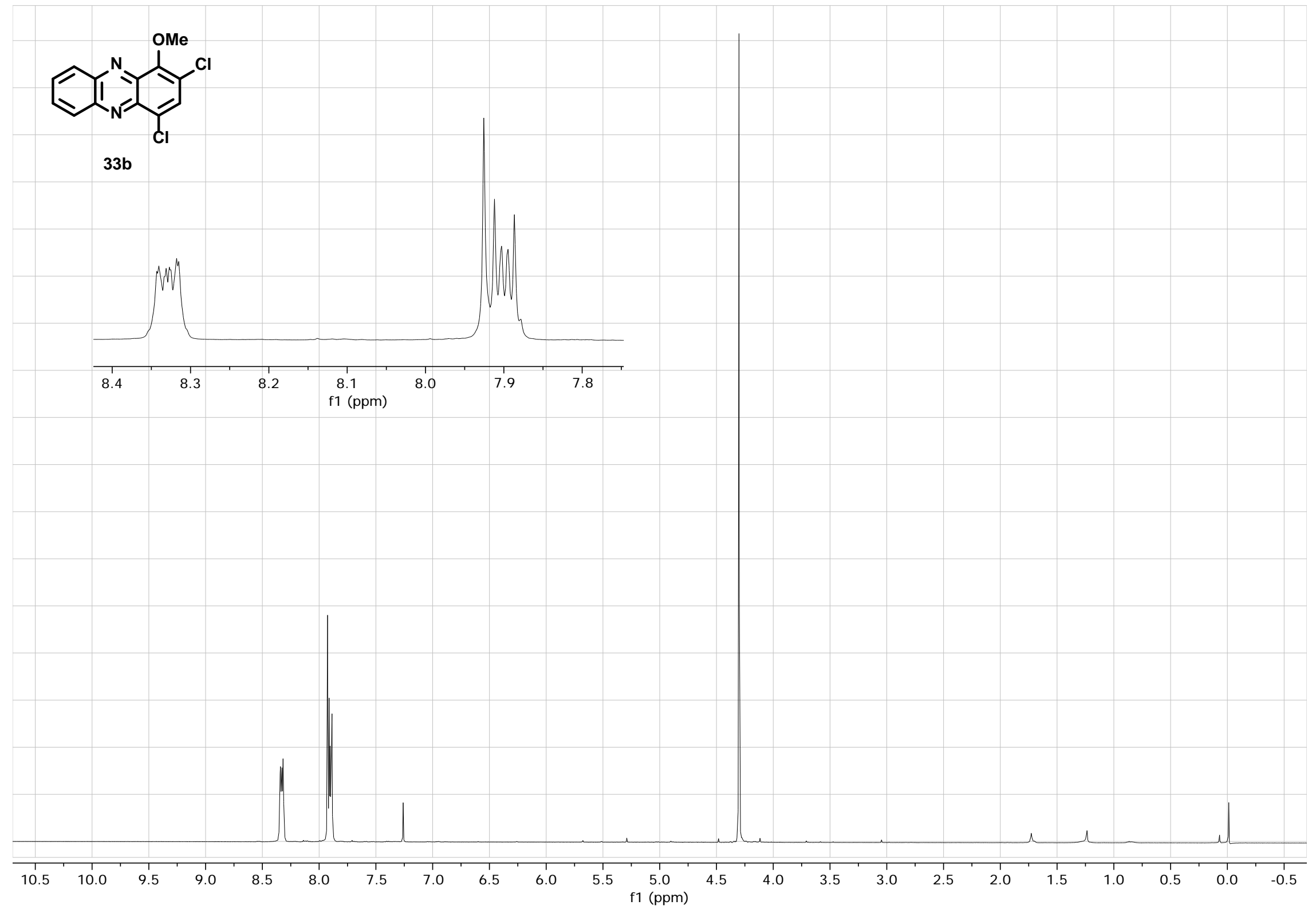




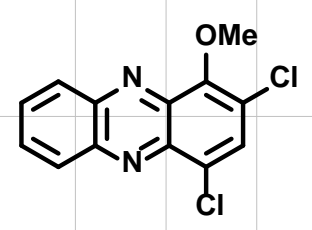

$33 b$

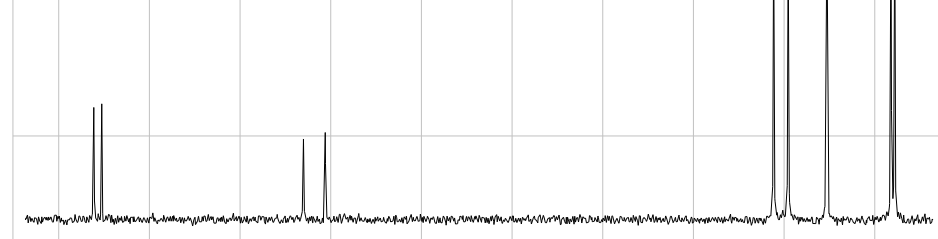

$\begin{array}{lllllllllllllll}144 & 143 & 142 & 141 & 140 & 139 & 138 & 137 & 136 & 135 & 134 & 133 & 132 & 131 & 130\end{array}$ f1 (ppm)

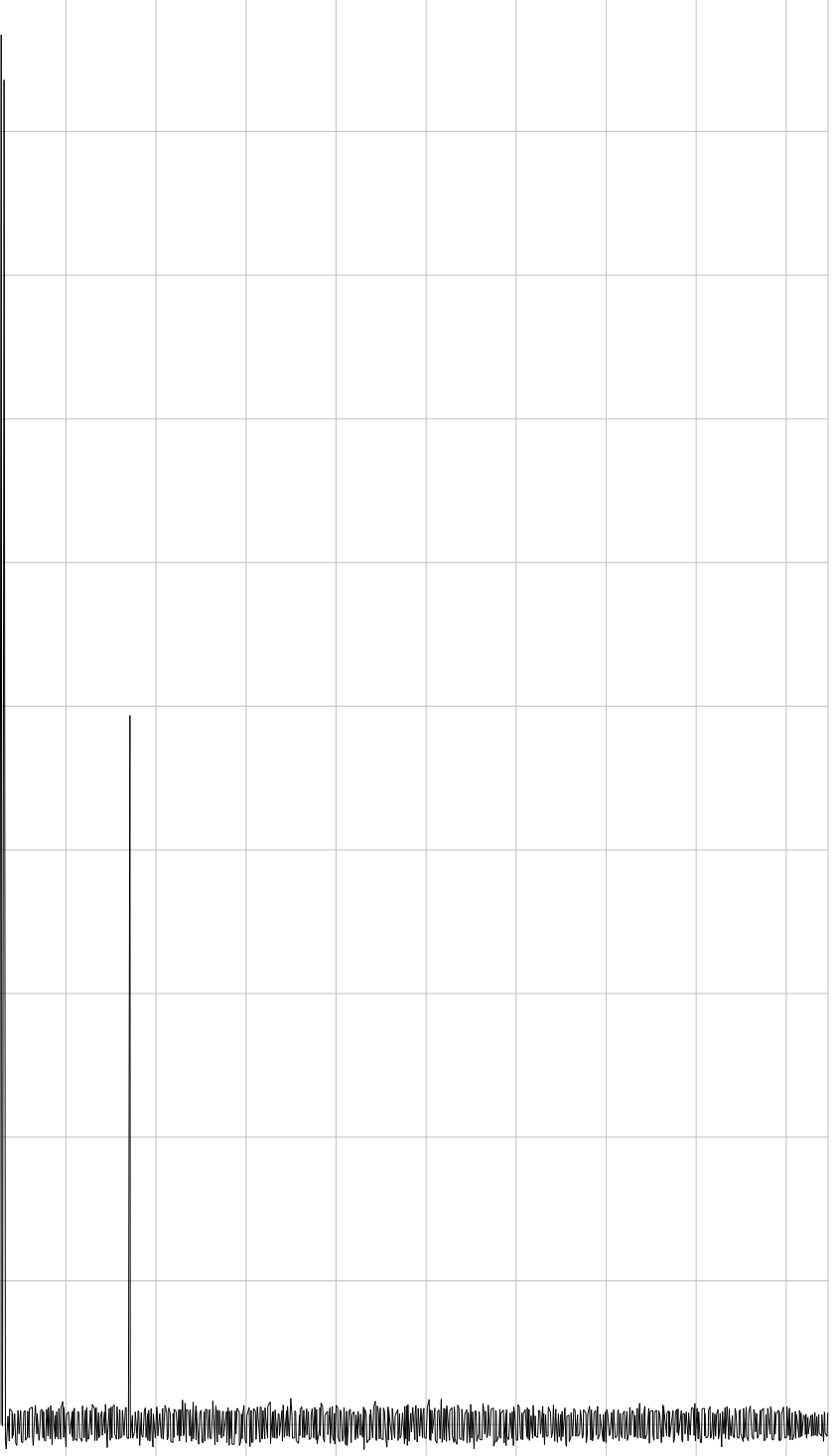

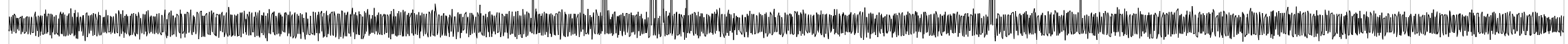

$\begin{array}{lllllllllllllllllllllllll}230 & 220 & 210 & 200 & 190 & 180 & 170 & 160 & 150 & 140 & 130 & 120 & \begin{array}{l}110 \\ \mathrm{f} 1(\mathrm{ppm})\end{array} & 100 & 90 & 80 & 70 & 60 & 50 & 40 & 30 & 20 & 10 & 0 & -10\end{array}$




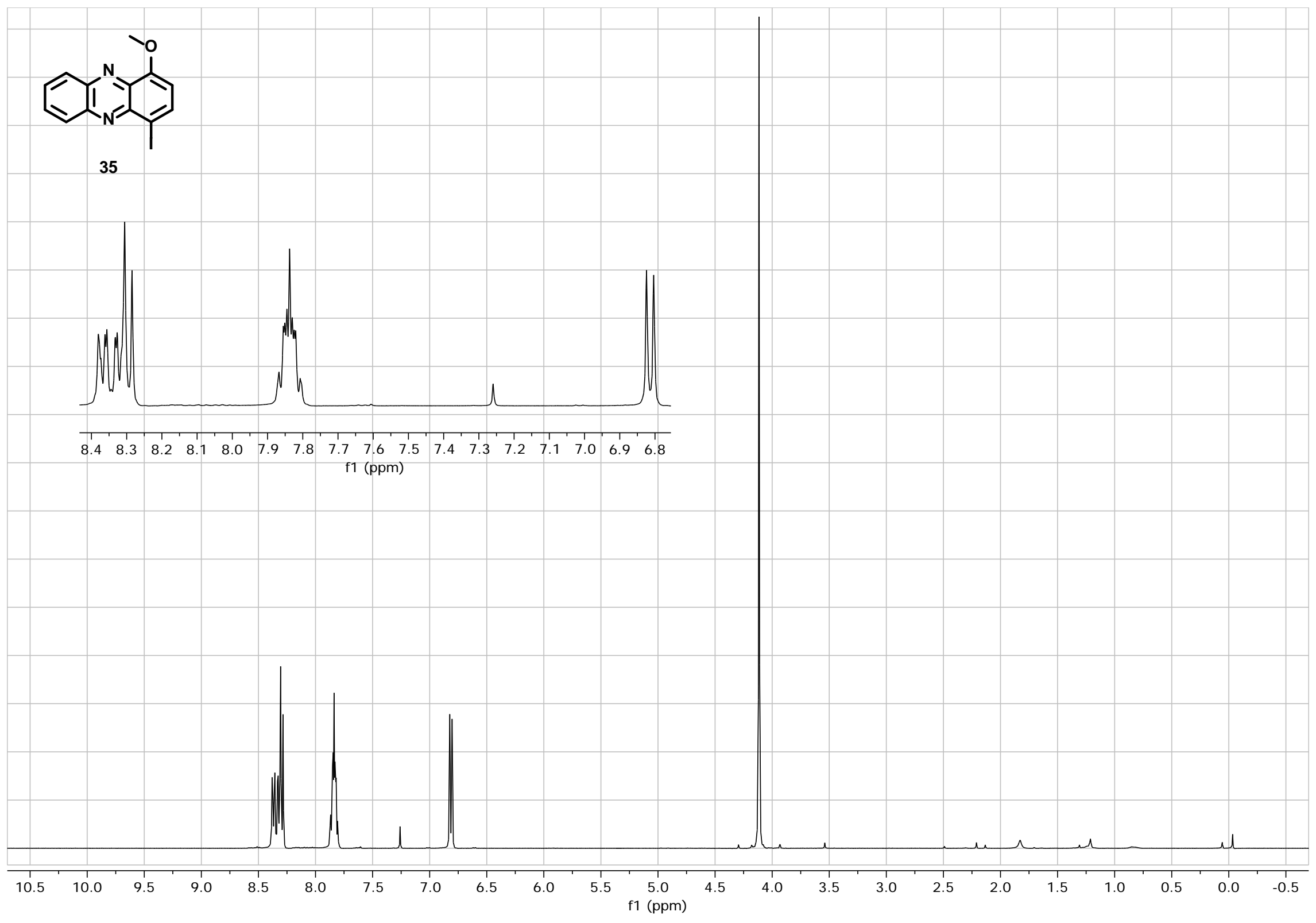




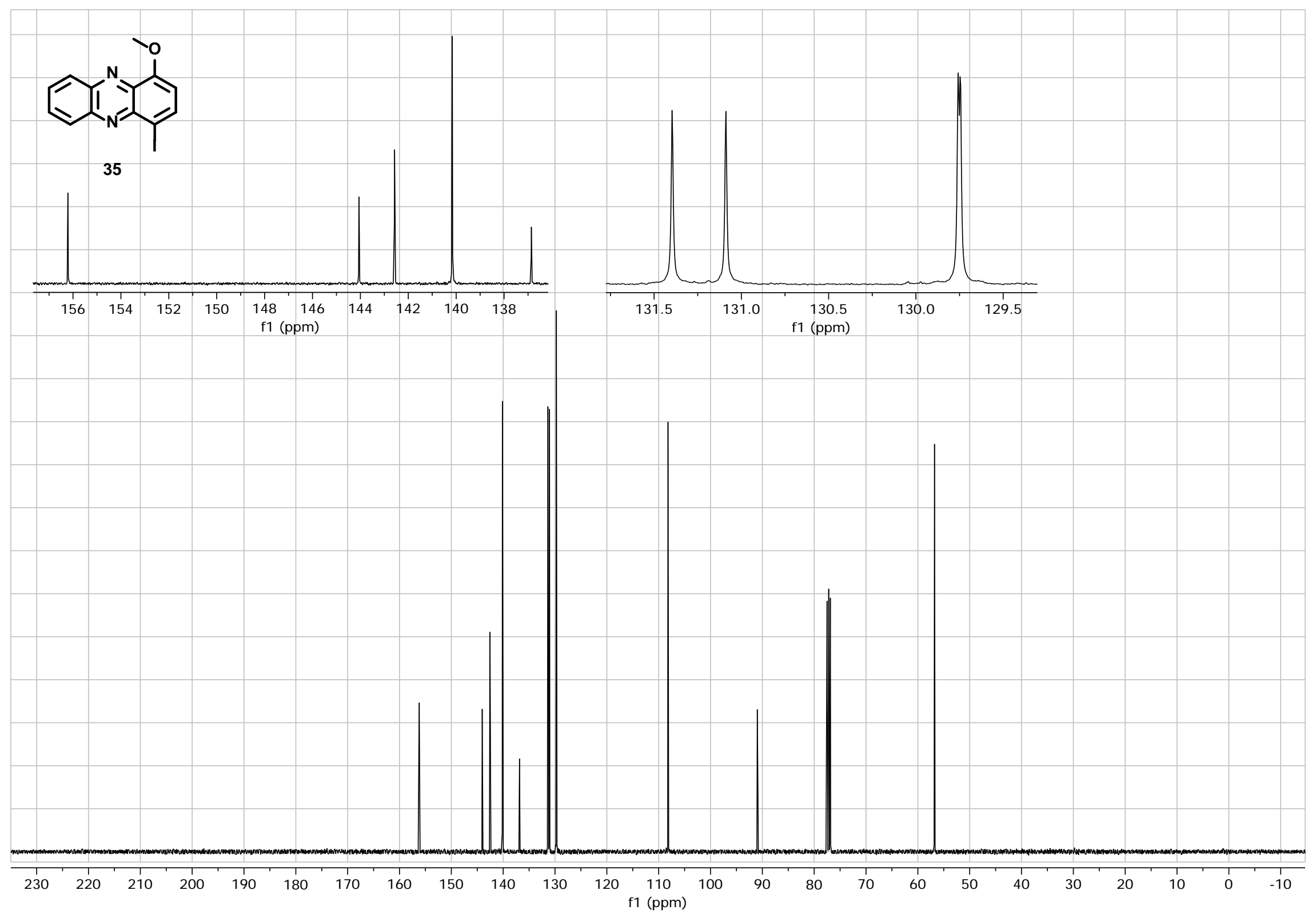




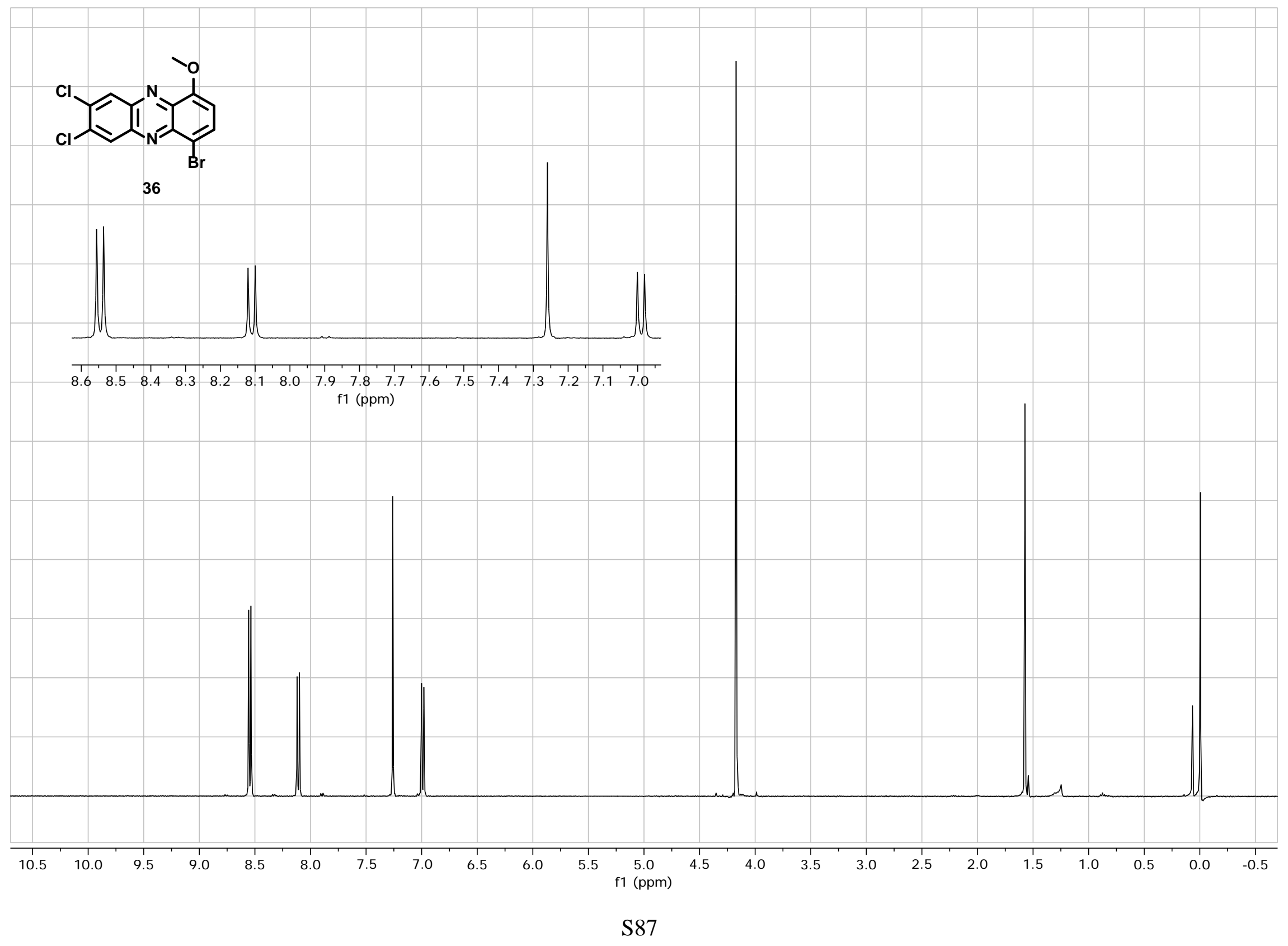




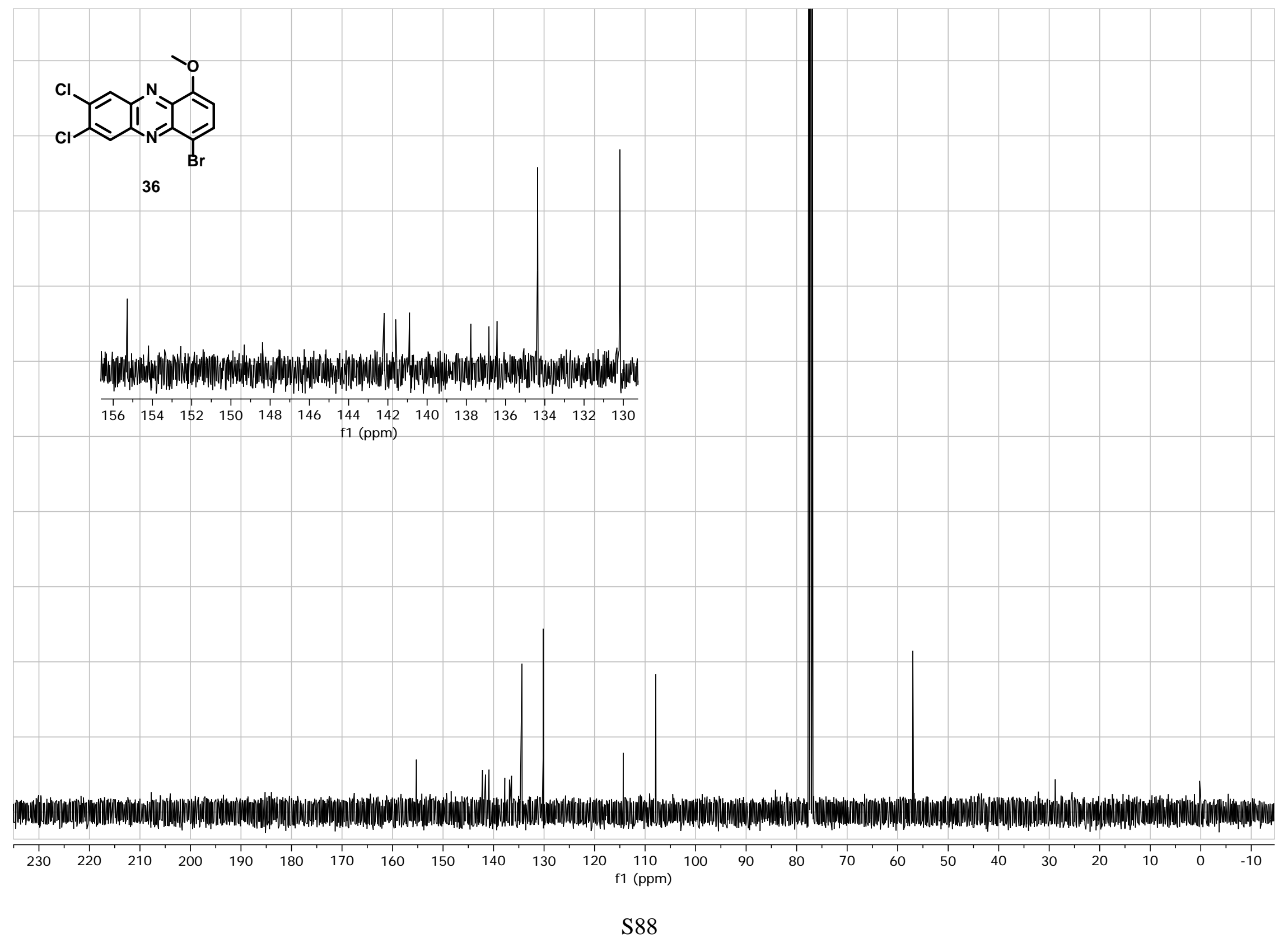




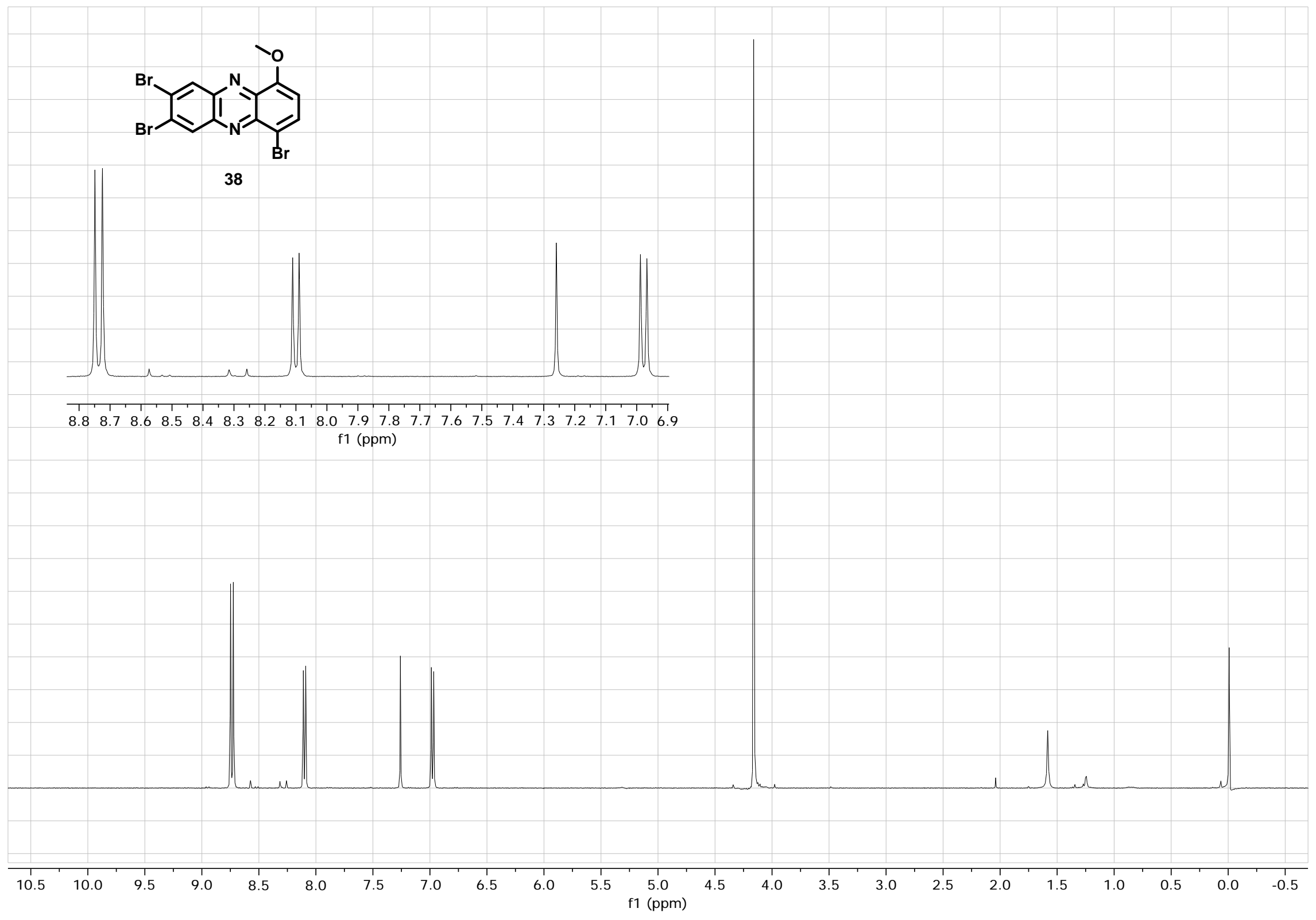




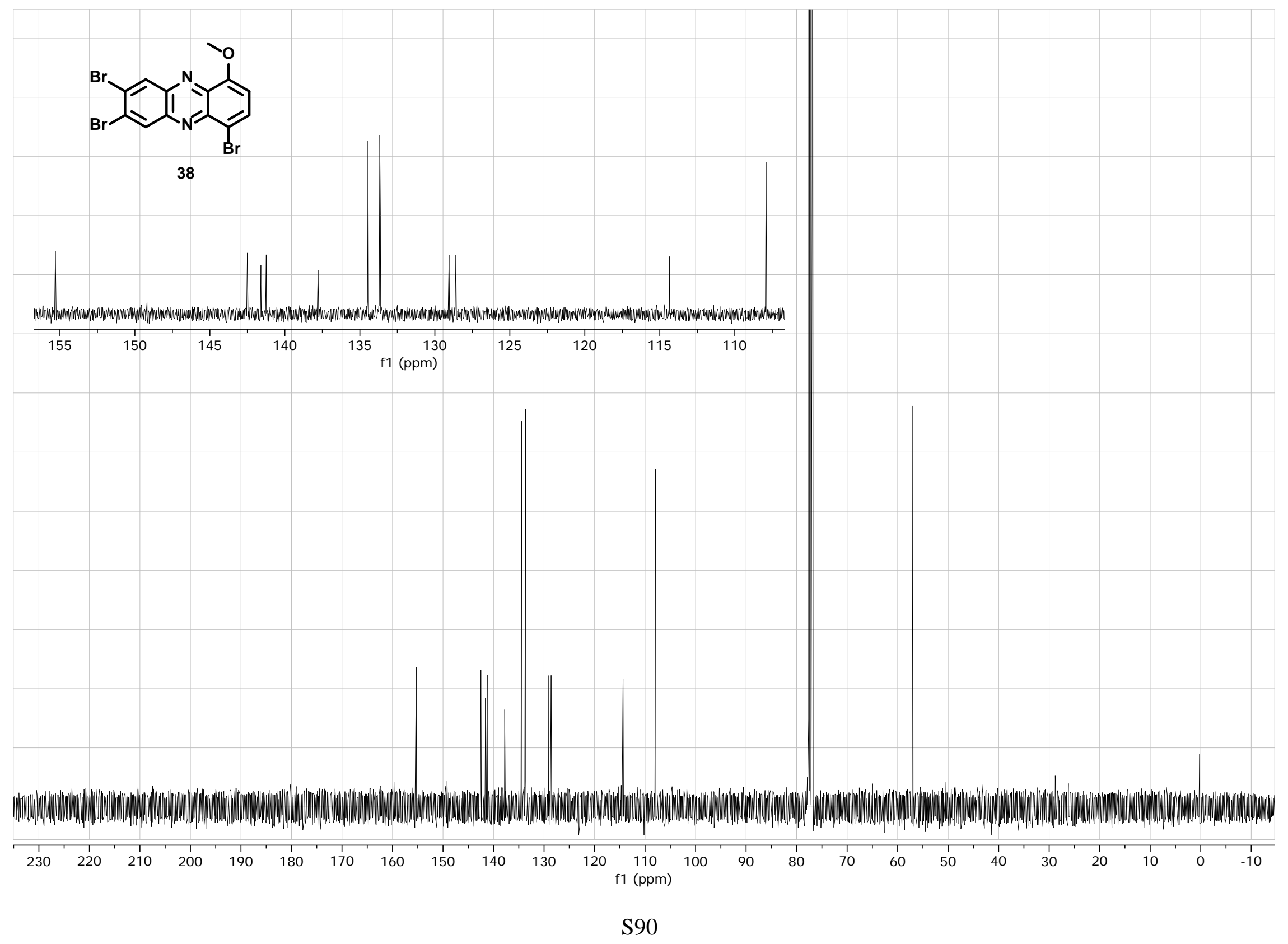



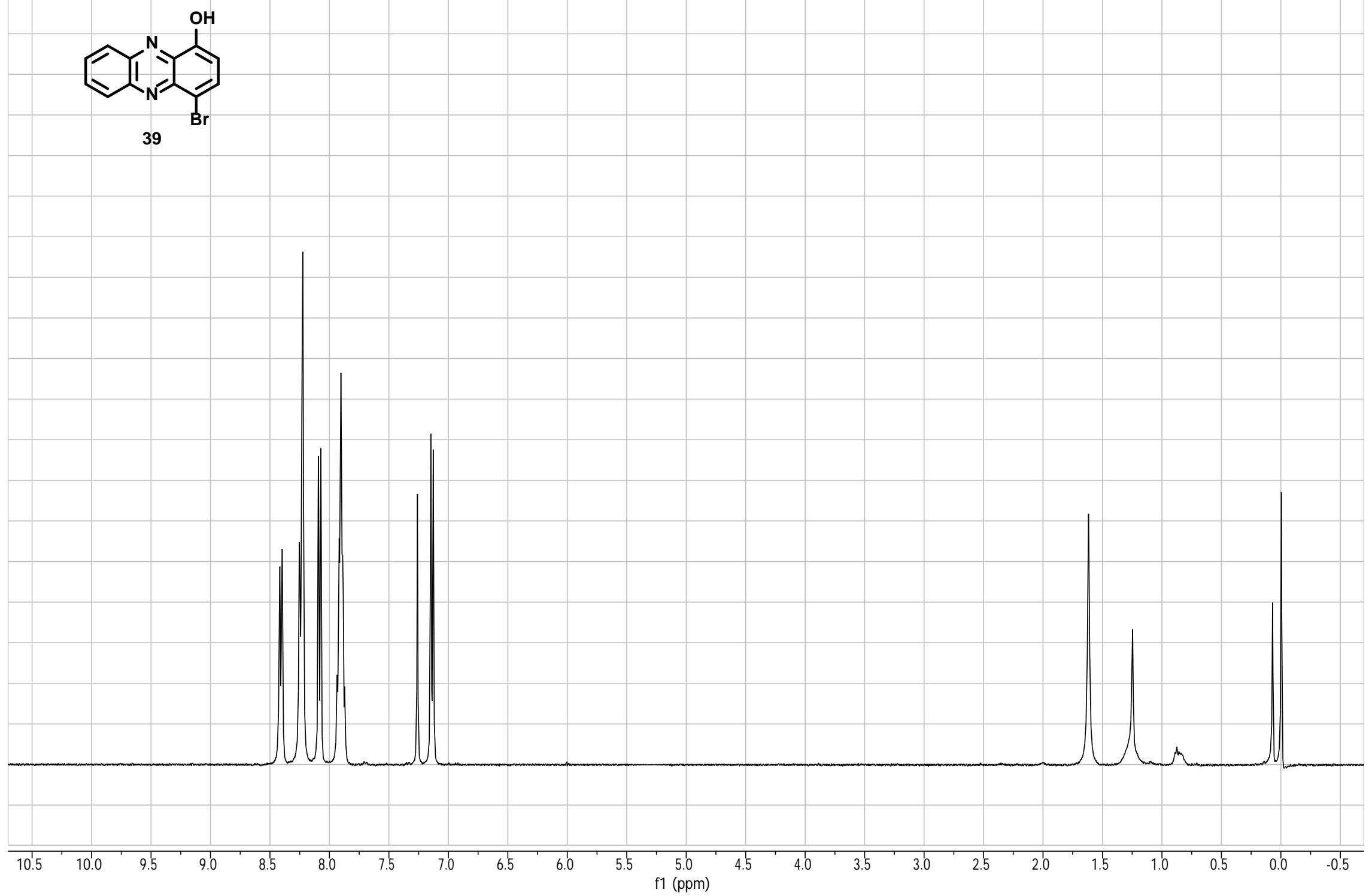

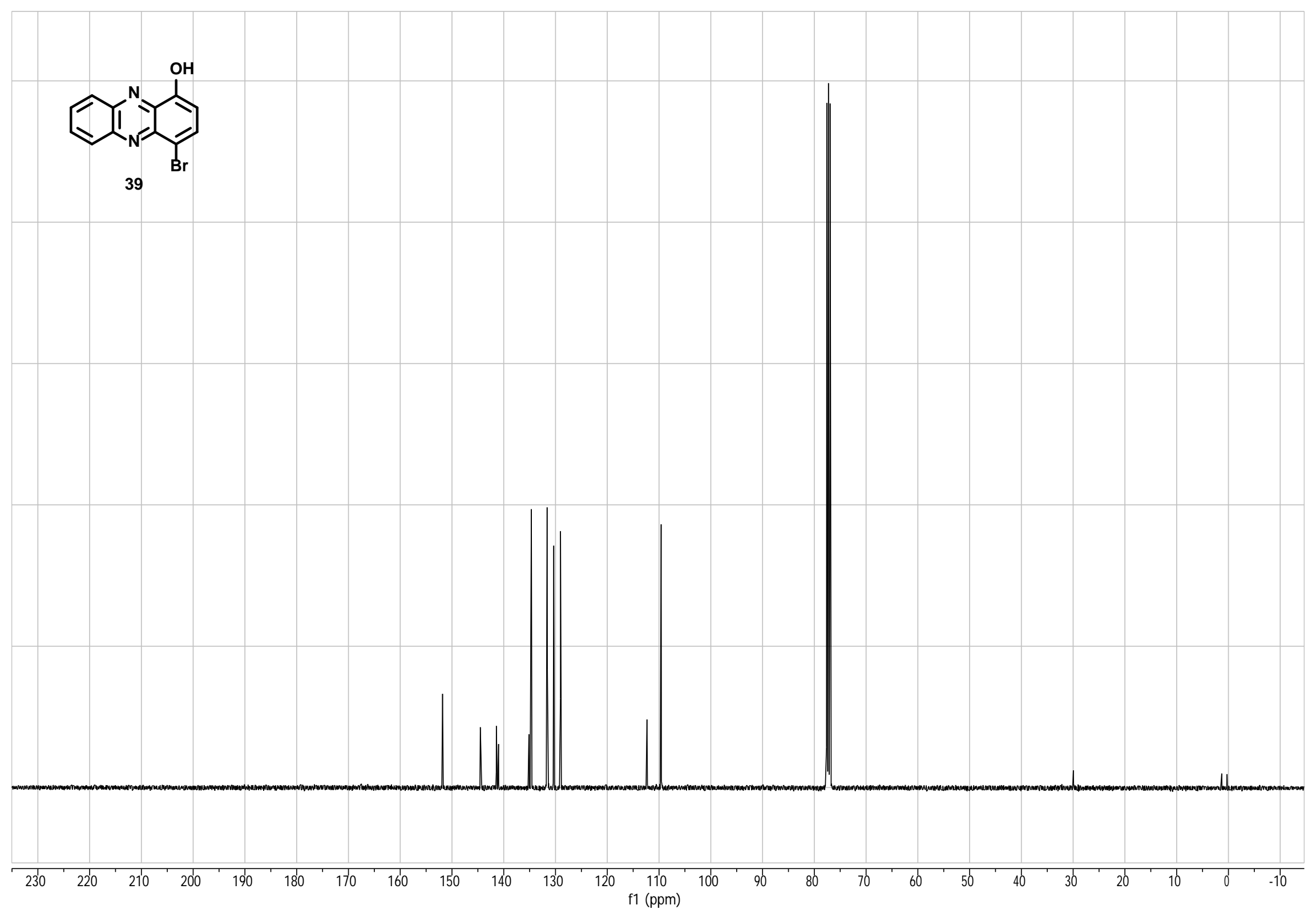


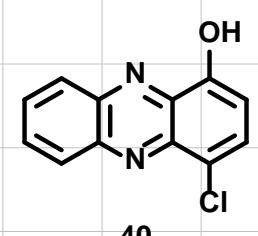

40
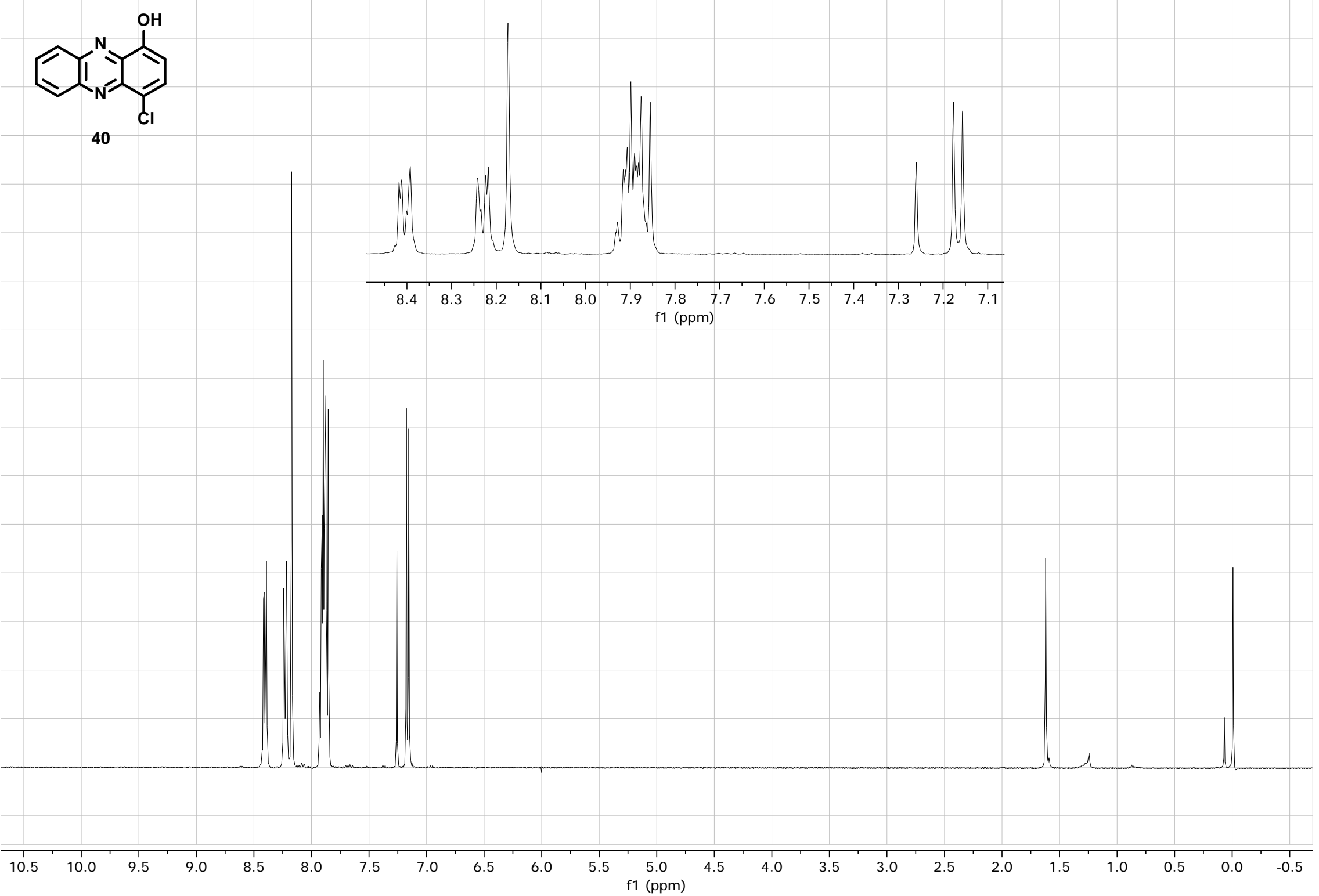


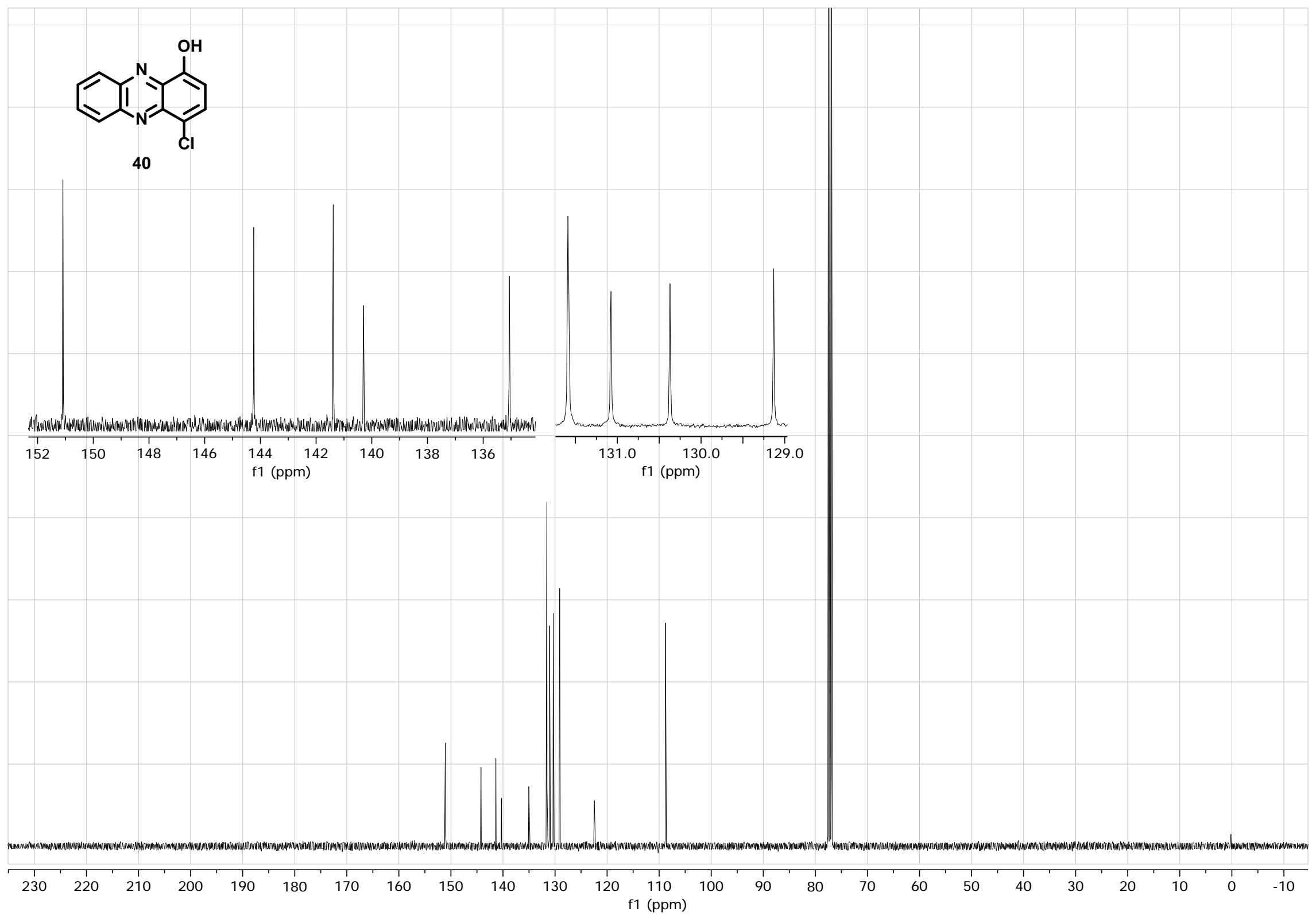




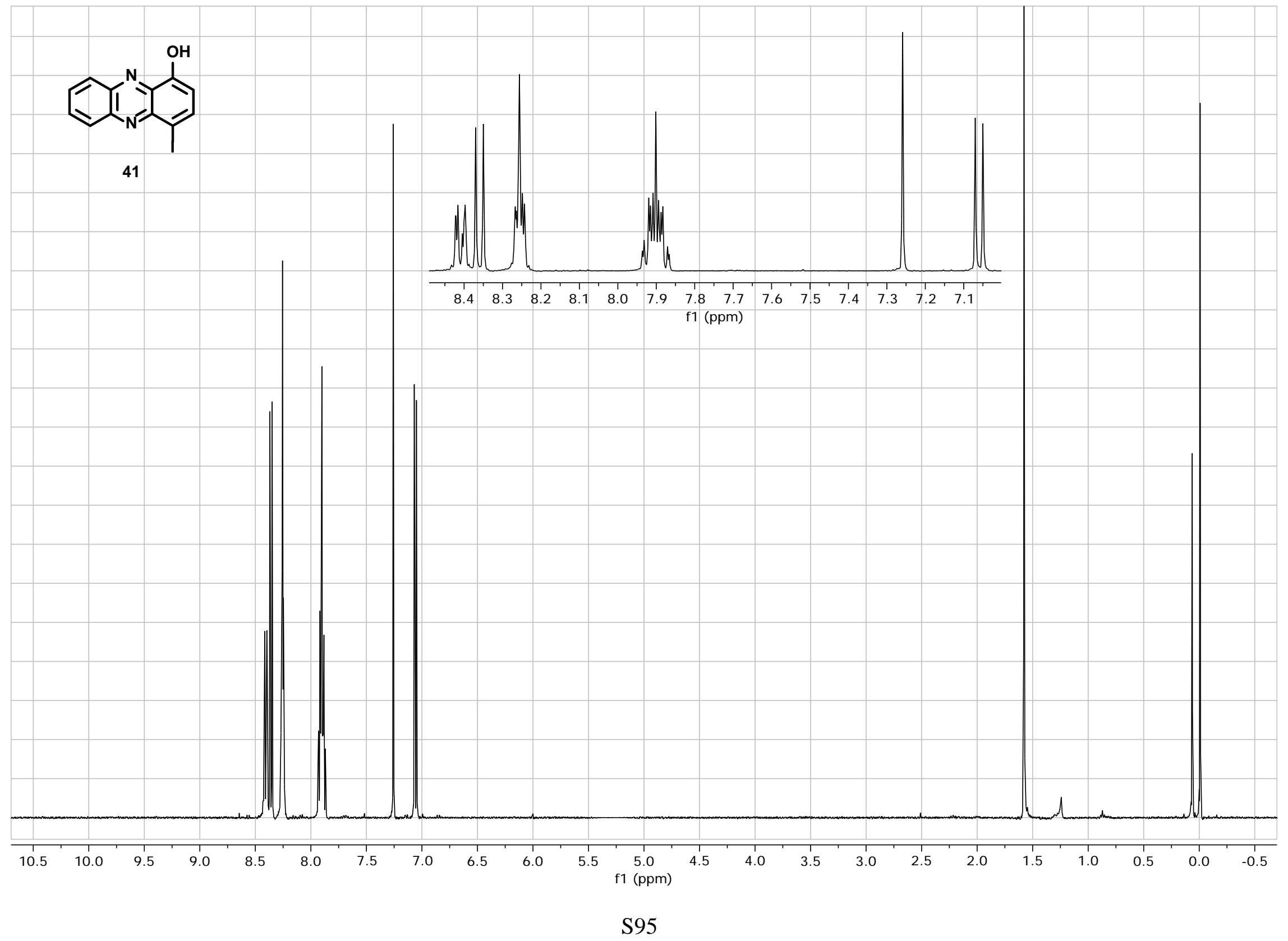




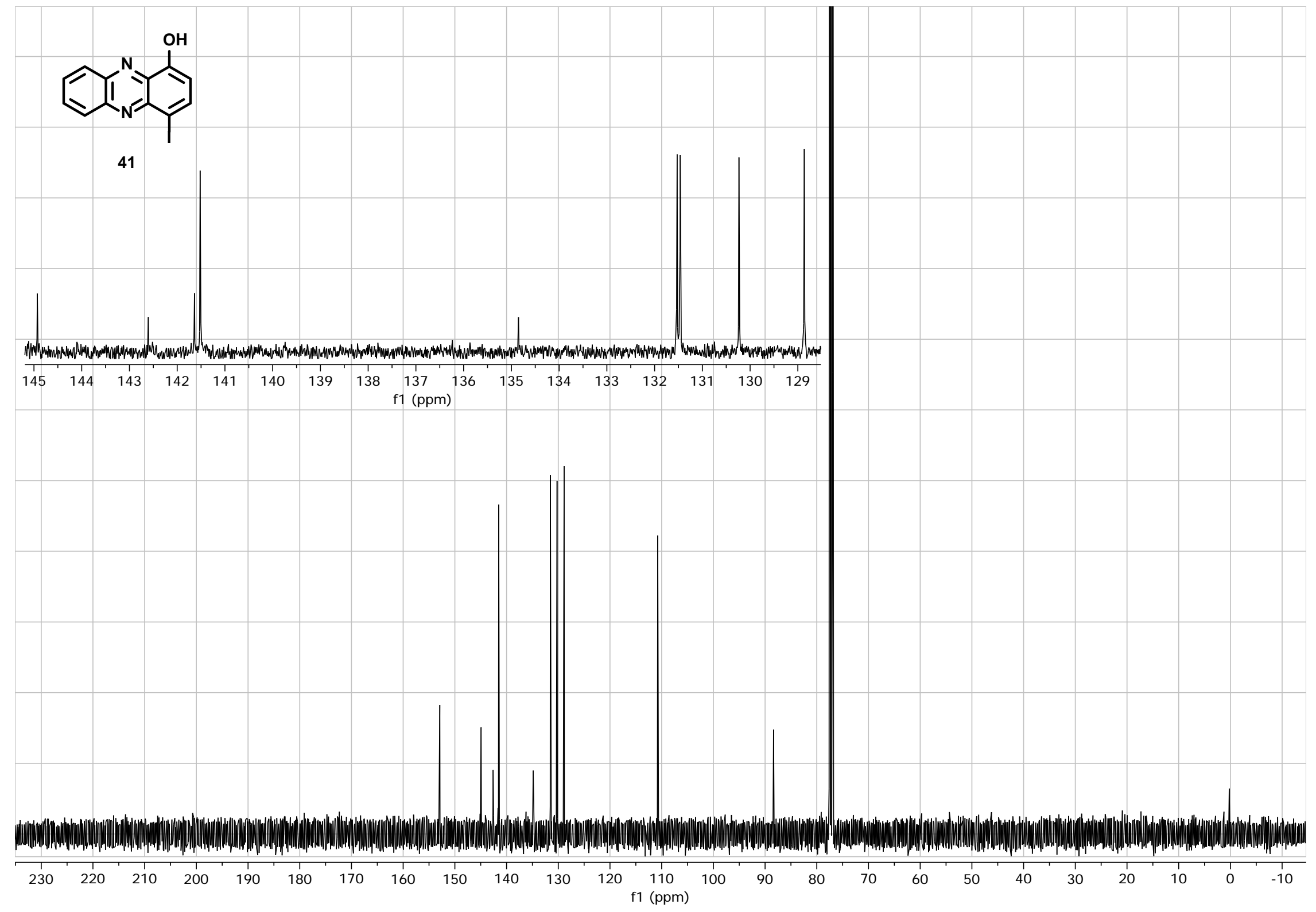




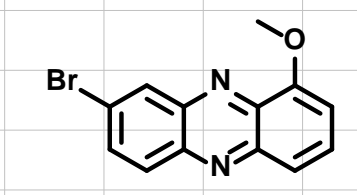

52
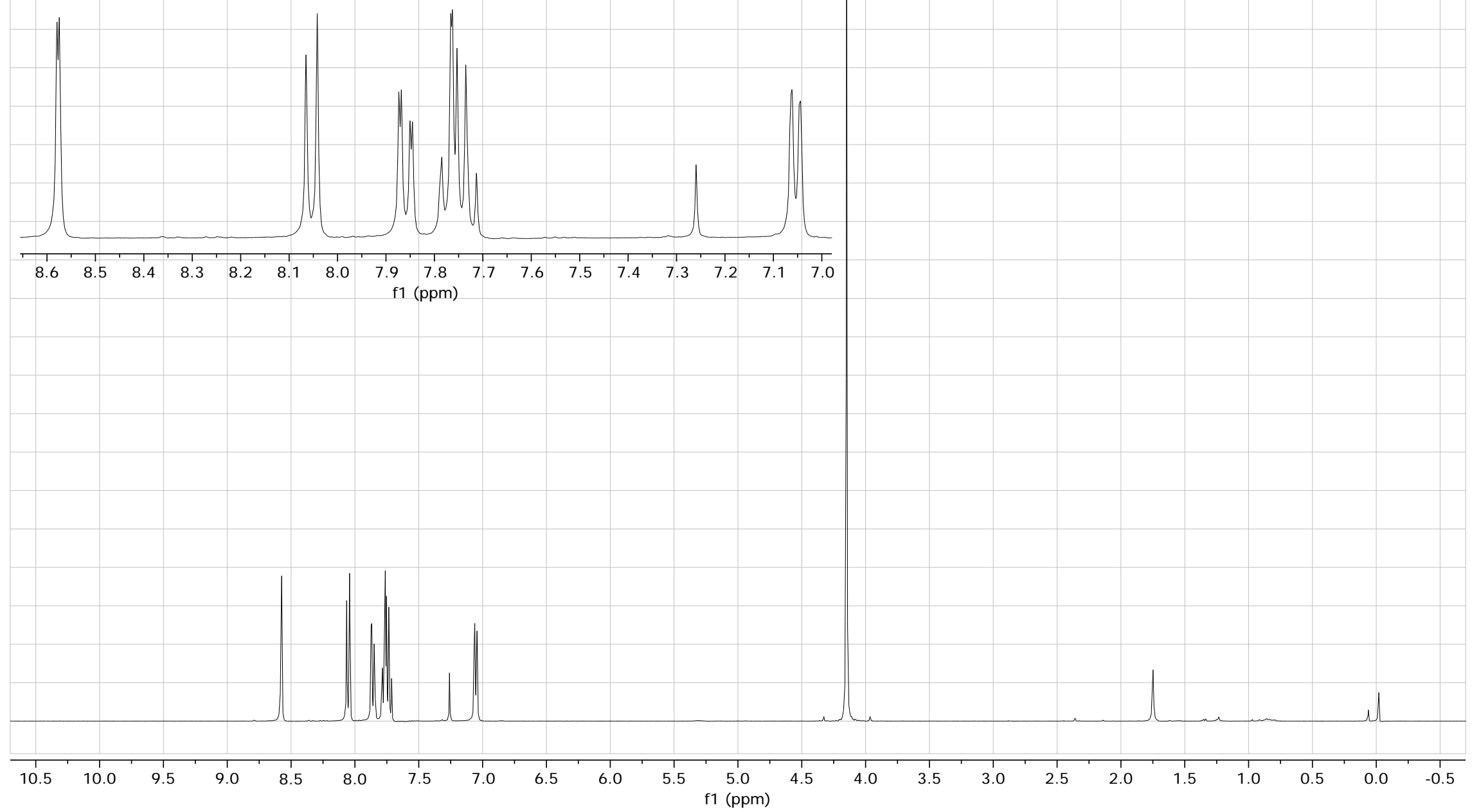


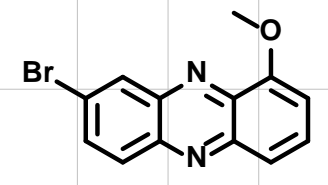

52

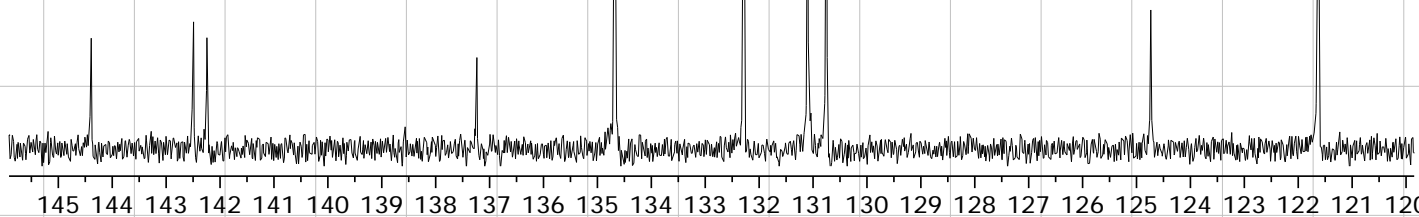
f1 (ppm)
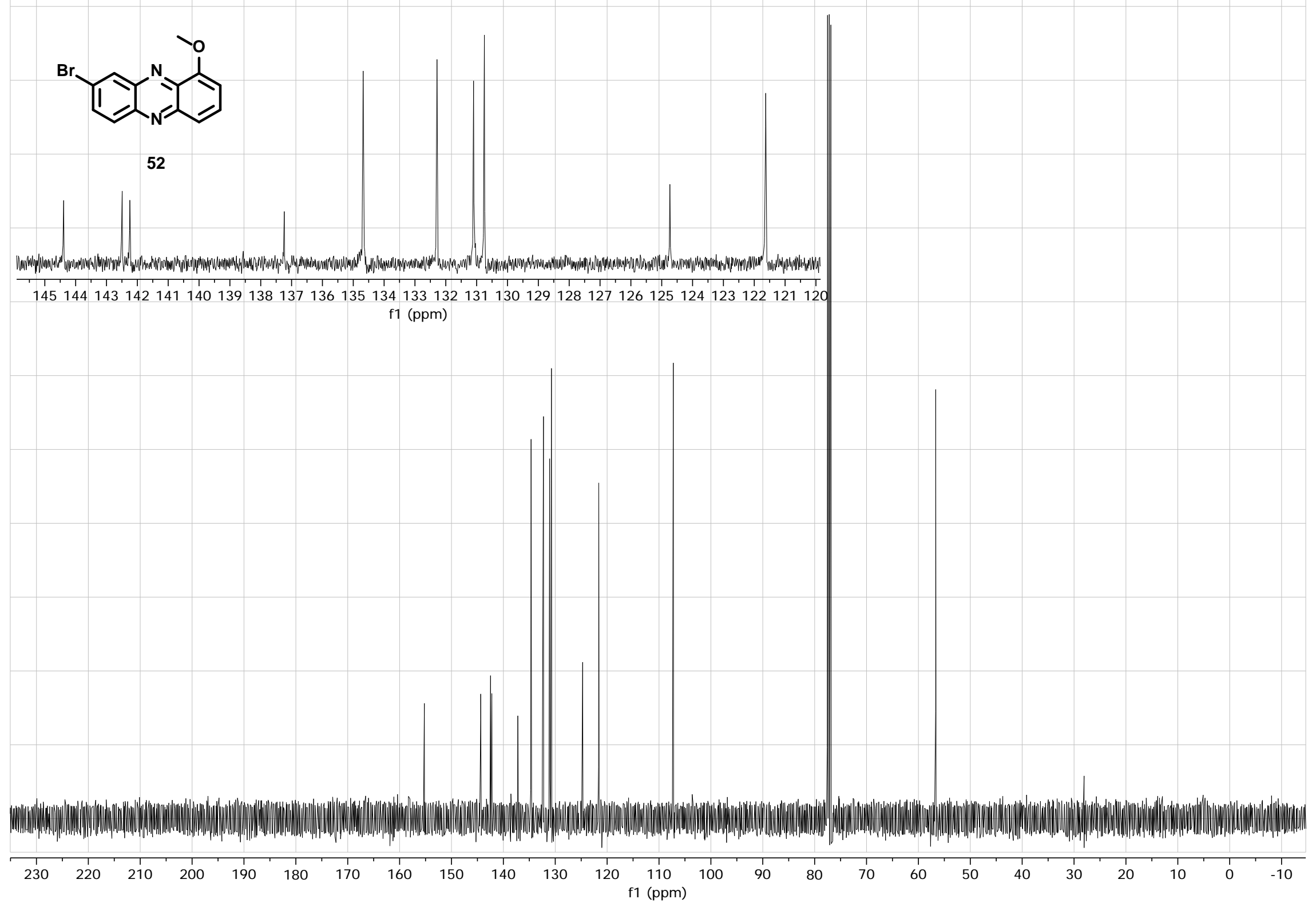


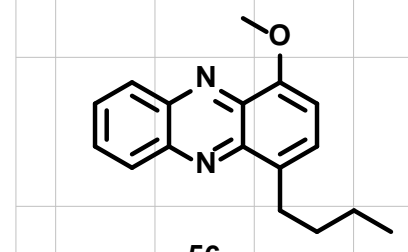

56
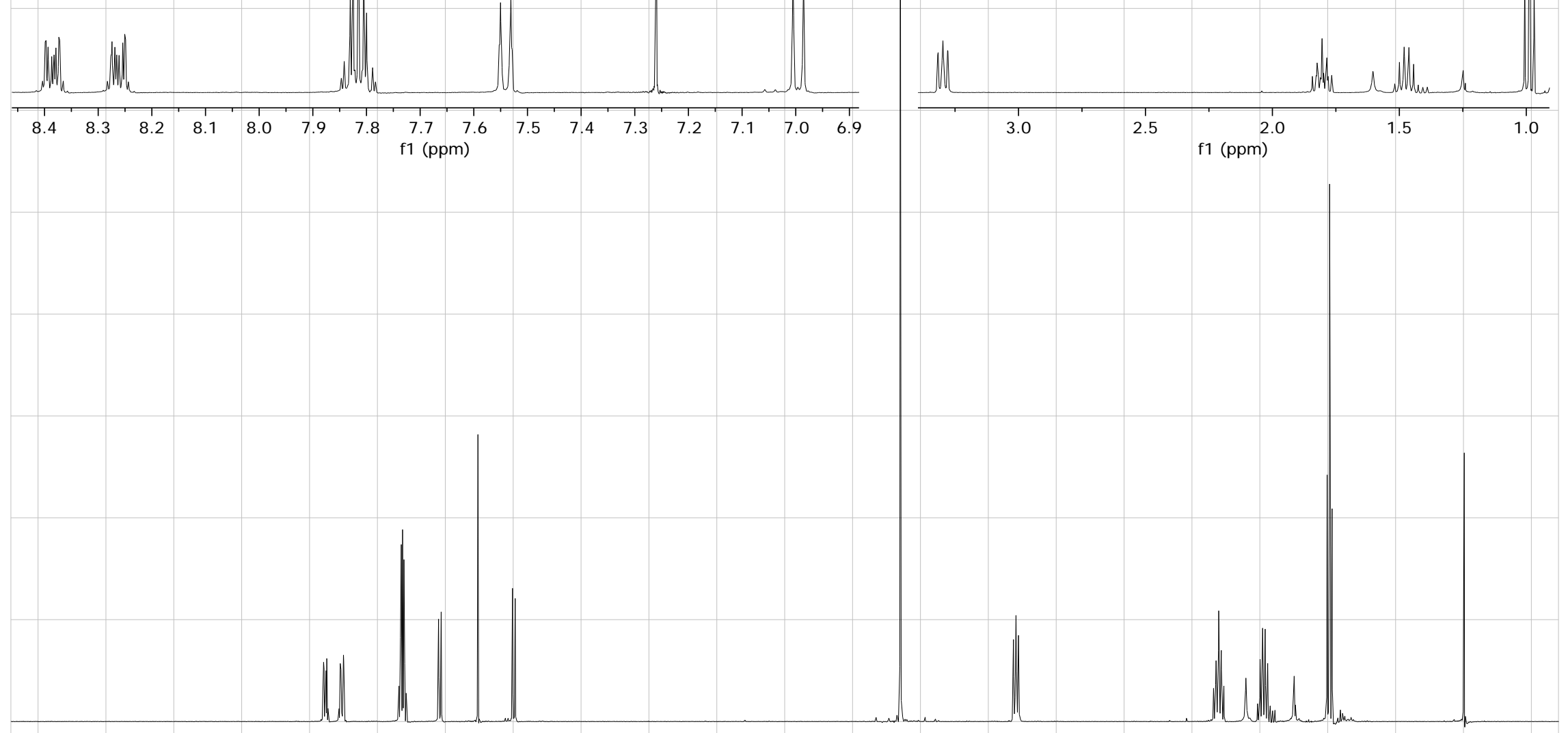

10.5

9.5

$9.0 \quad 8.5$
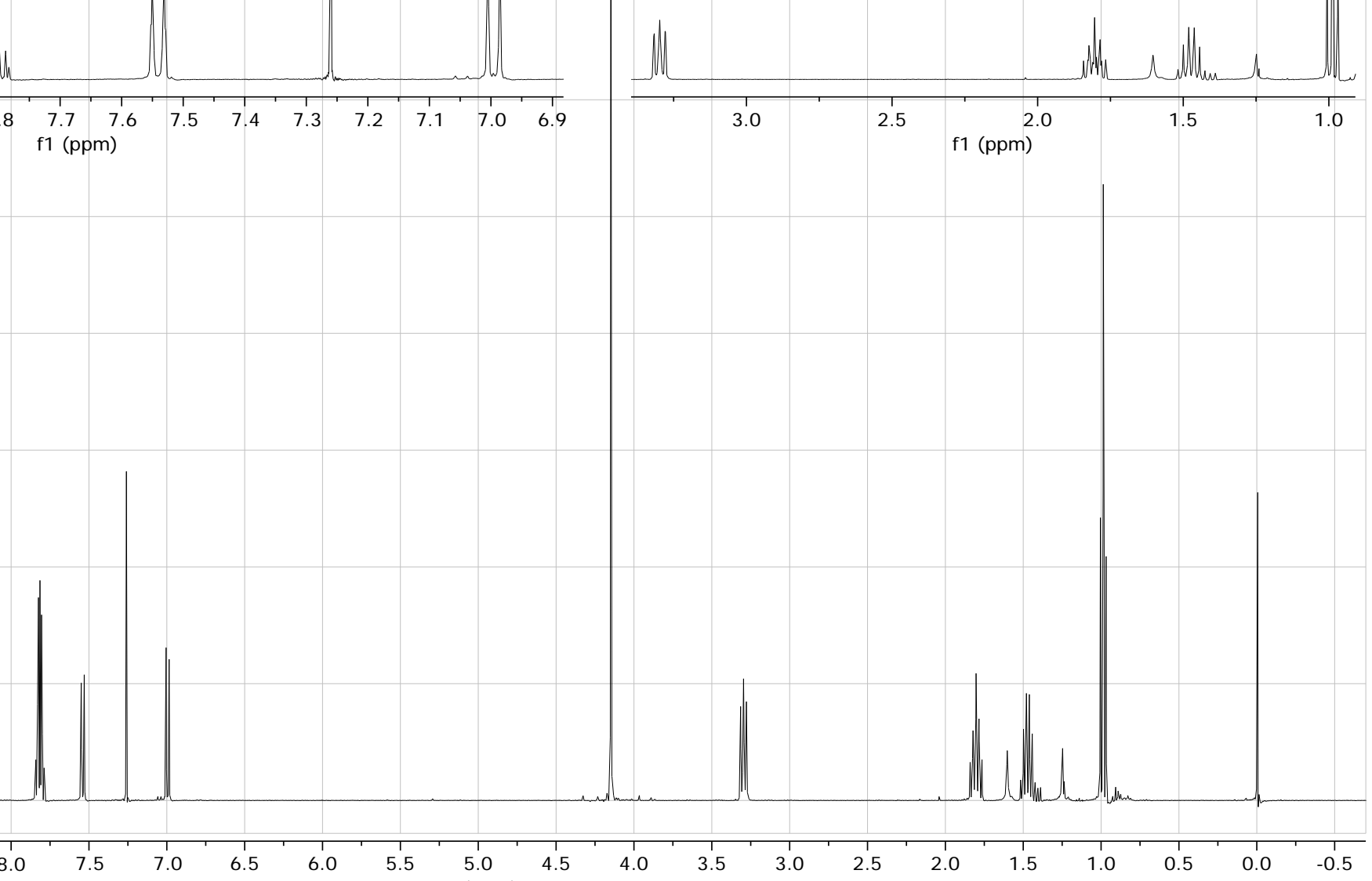

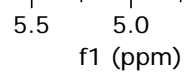

4.54.

S99 


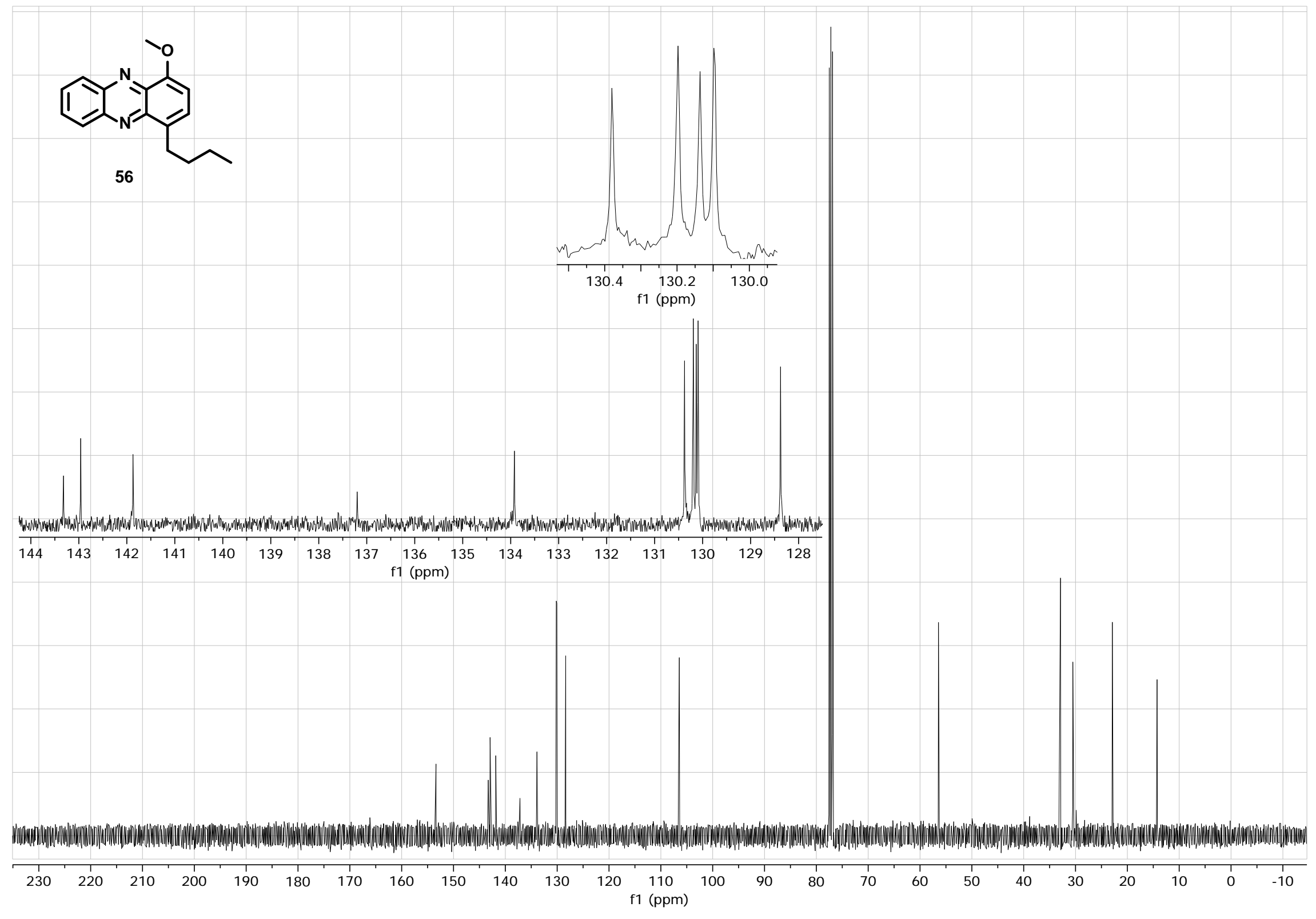




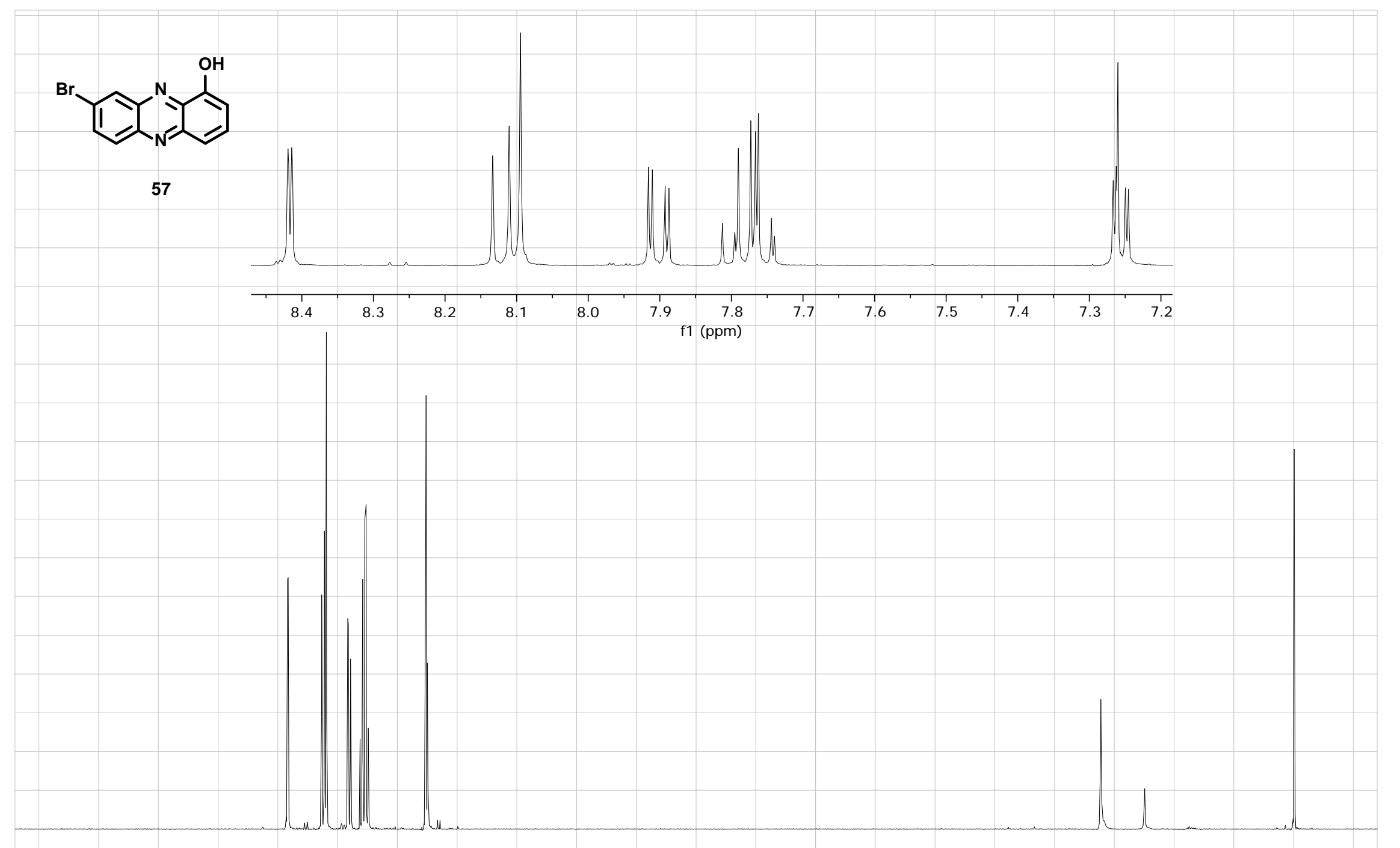

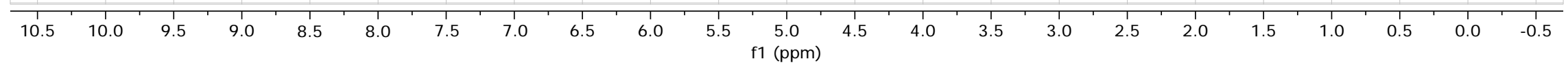




\section{(1)}

57

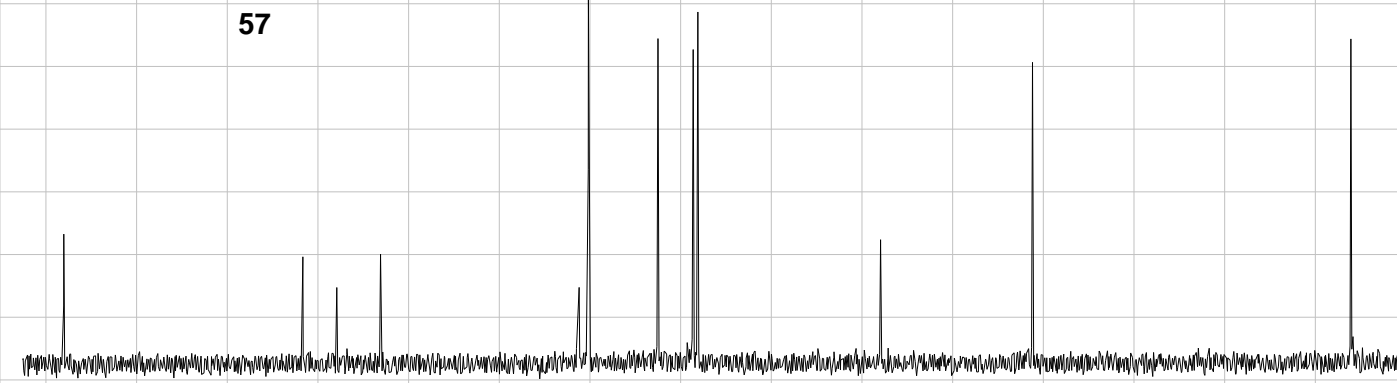

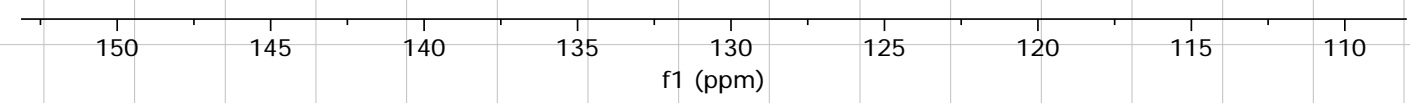

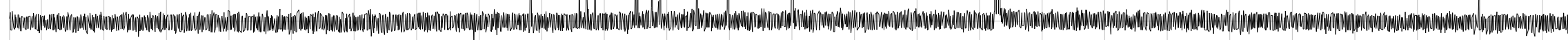



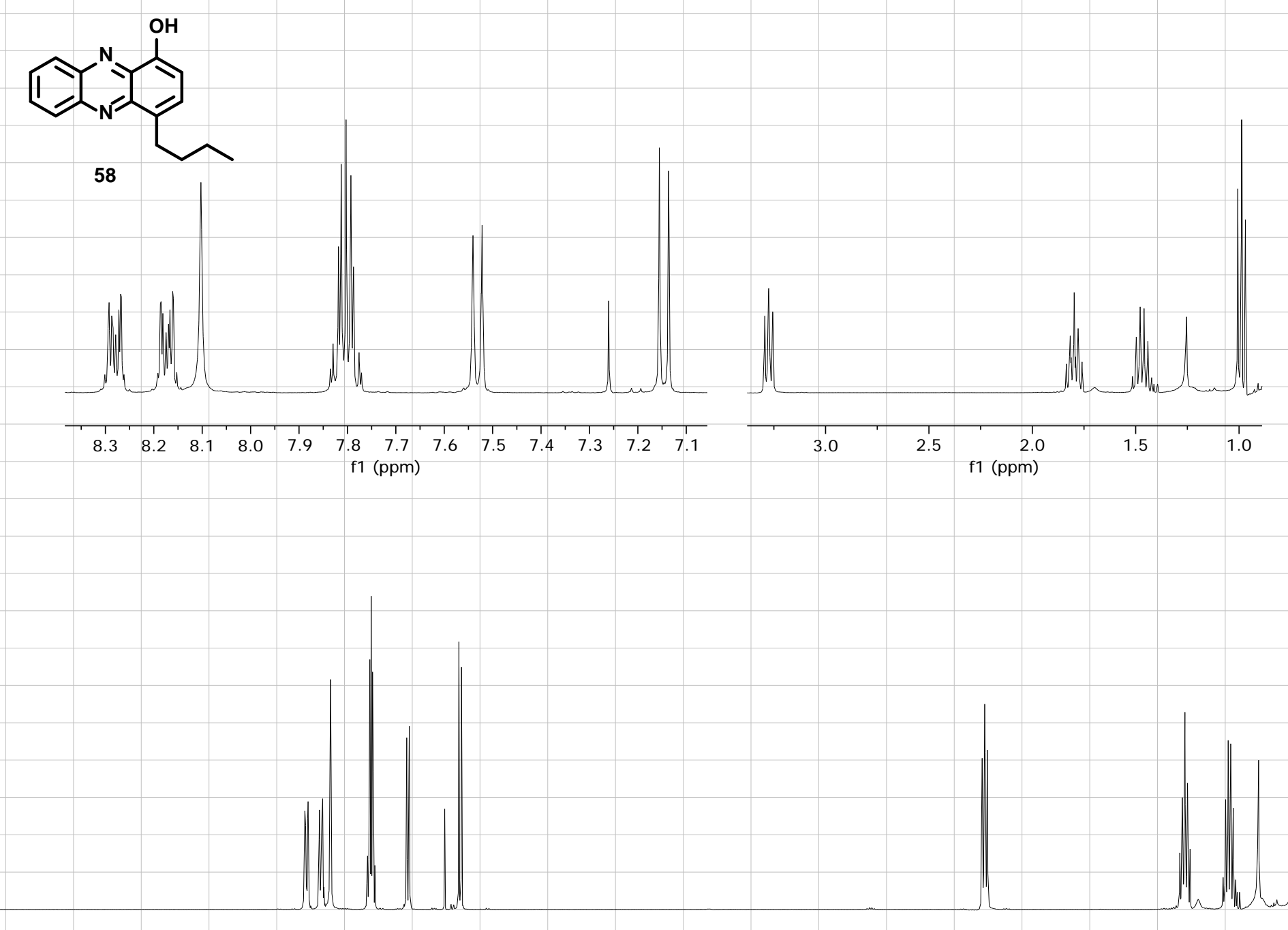

\begin{tabular}{lllllllllllllllllllllll}
\hline 10.5 & 10.0 & 9.5 & 9.0 & 8.5 & 8.0 & 7.5 & 7.0 & 6.5 & 6.0 & 5.5 & $\begin{array}{c}1 \\
\mathrm{f} 1.0 \\
(\mathrm{ppm})\end{array}$ & 4.5 & 4.0 & 3.5 & 3.0 & 2.5 & 2.0 & 1.5 & 1.0 & 0.5 & 0.0 & -0.5
\end{tabular}




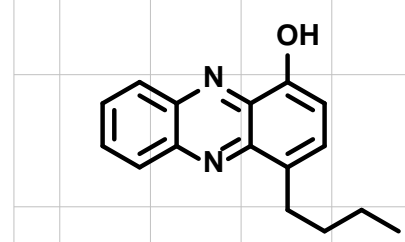

58

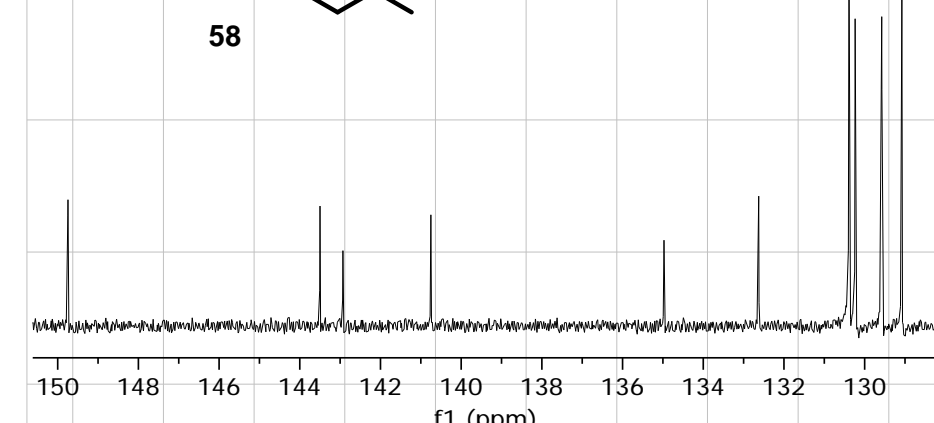

$\begin{array}{lllllll}150 & 148 & 146 & 144 & 142 & 140 & 138\end{array}$

f1 (ppm)

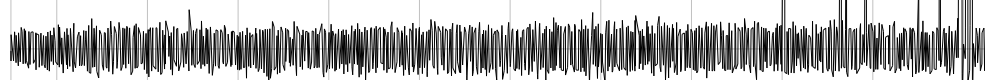

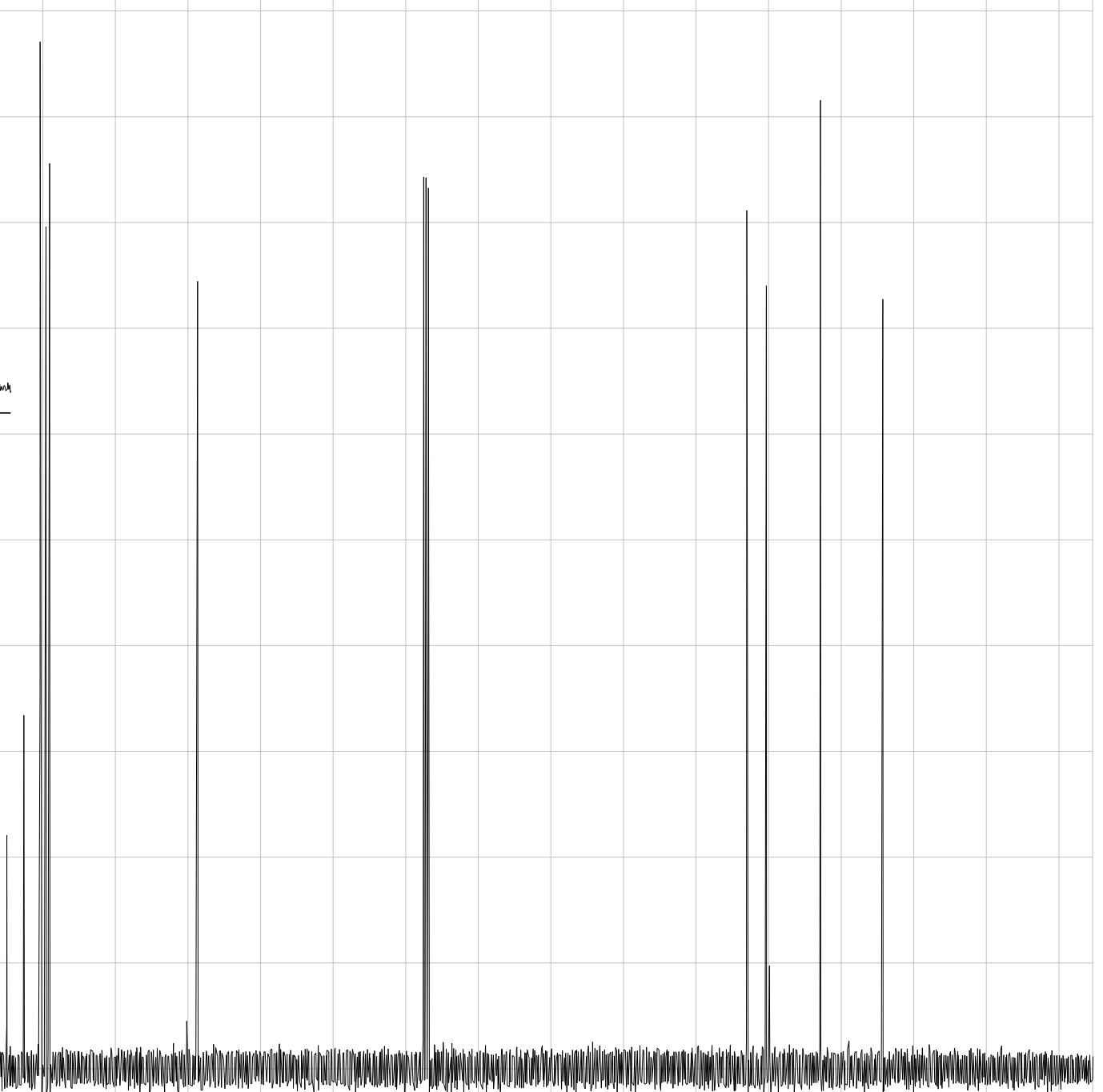

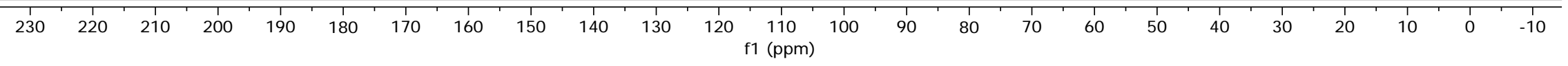

S104 


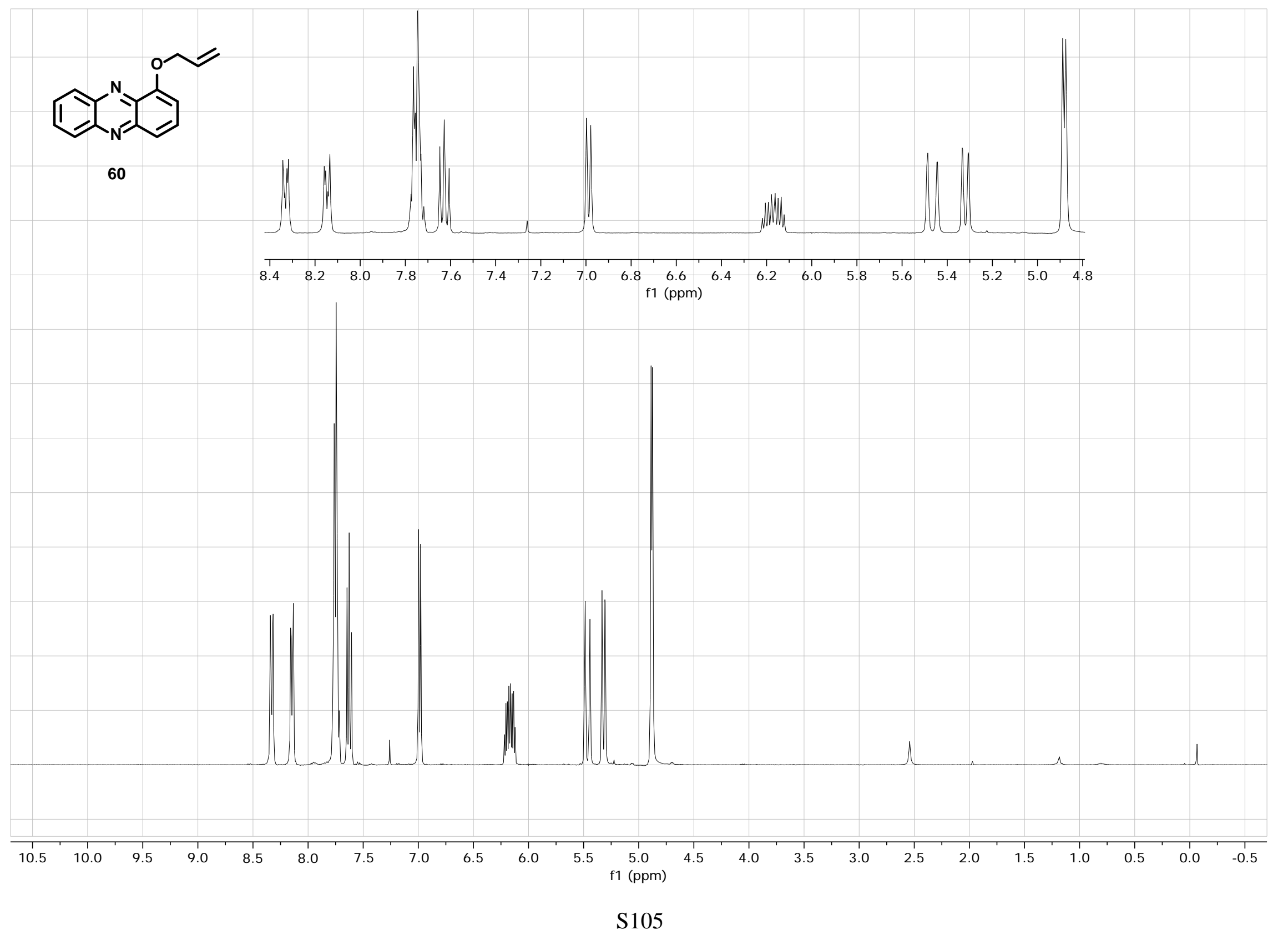




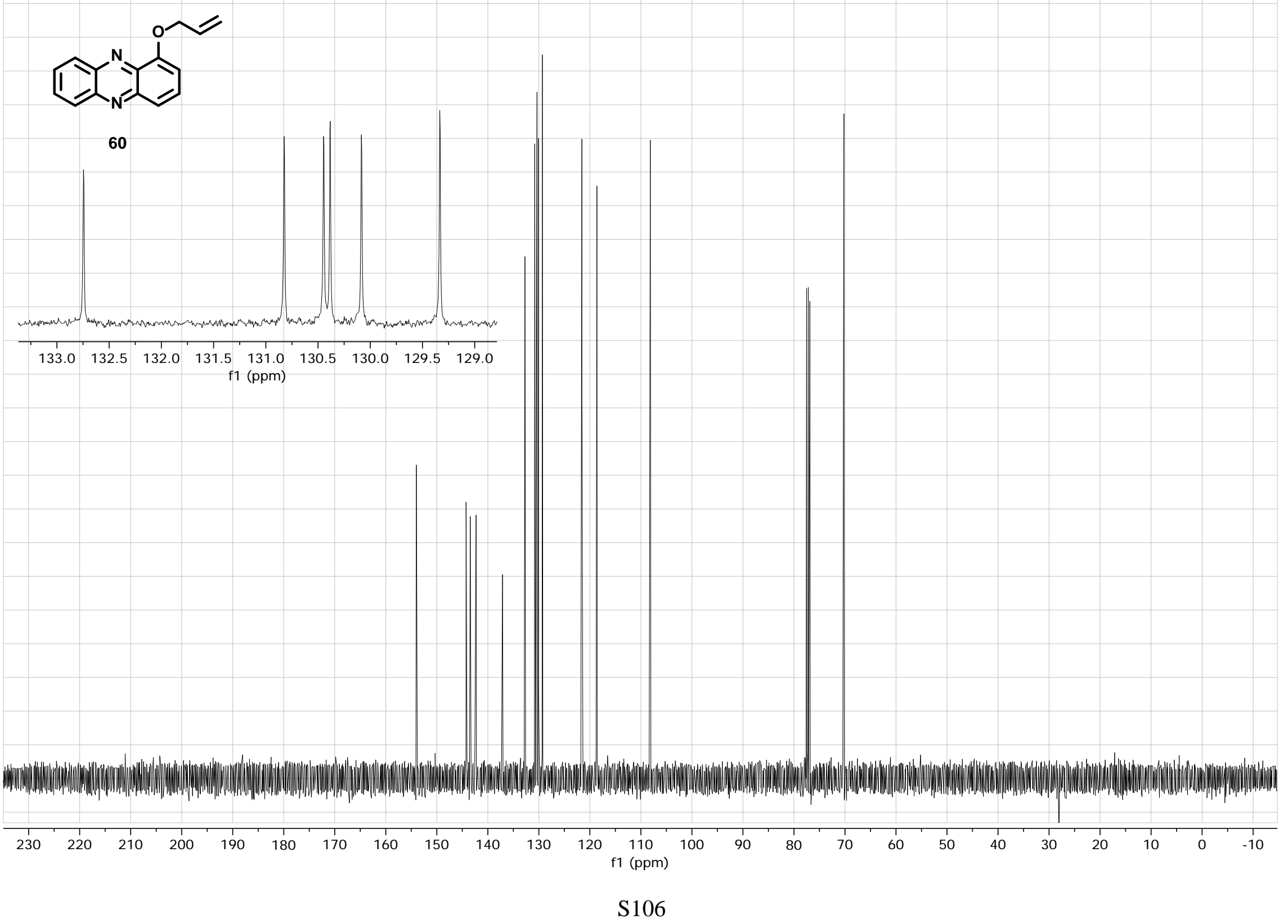




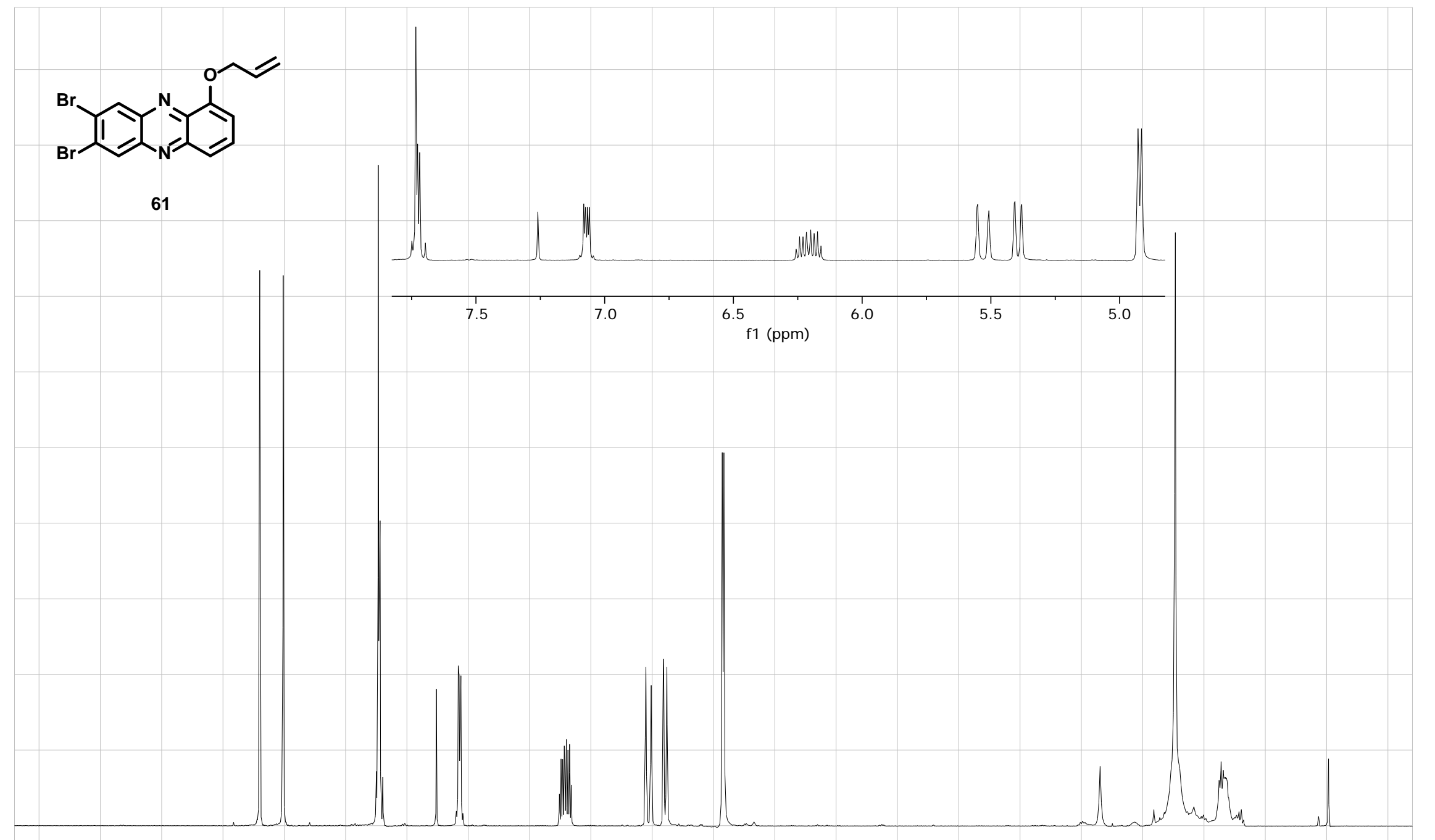

10.510 .0

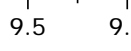

$9.0 \quad 8$
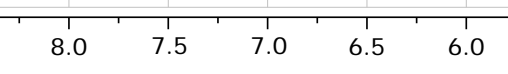


\section{(I)}

61

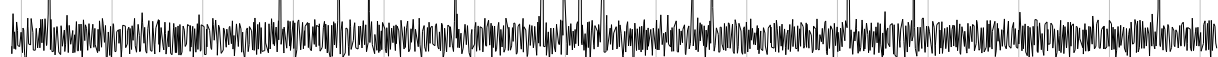

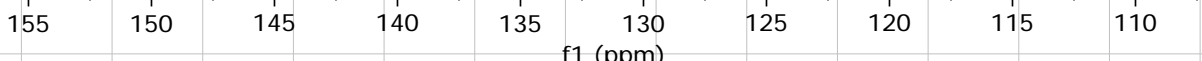

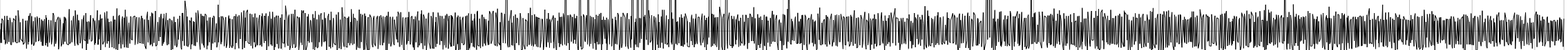




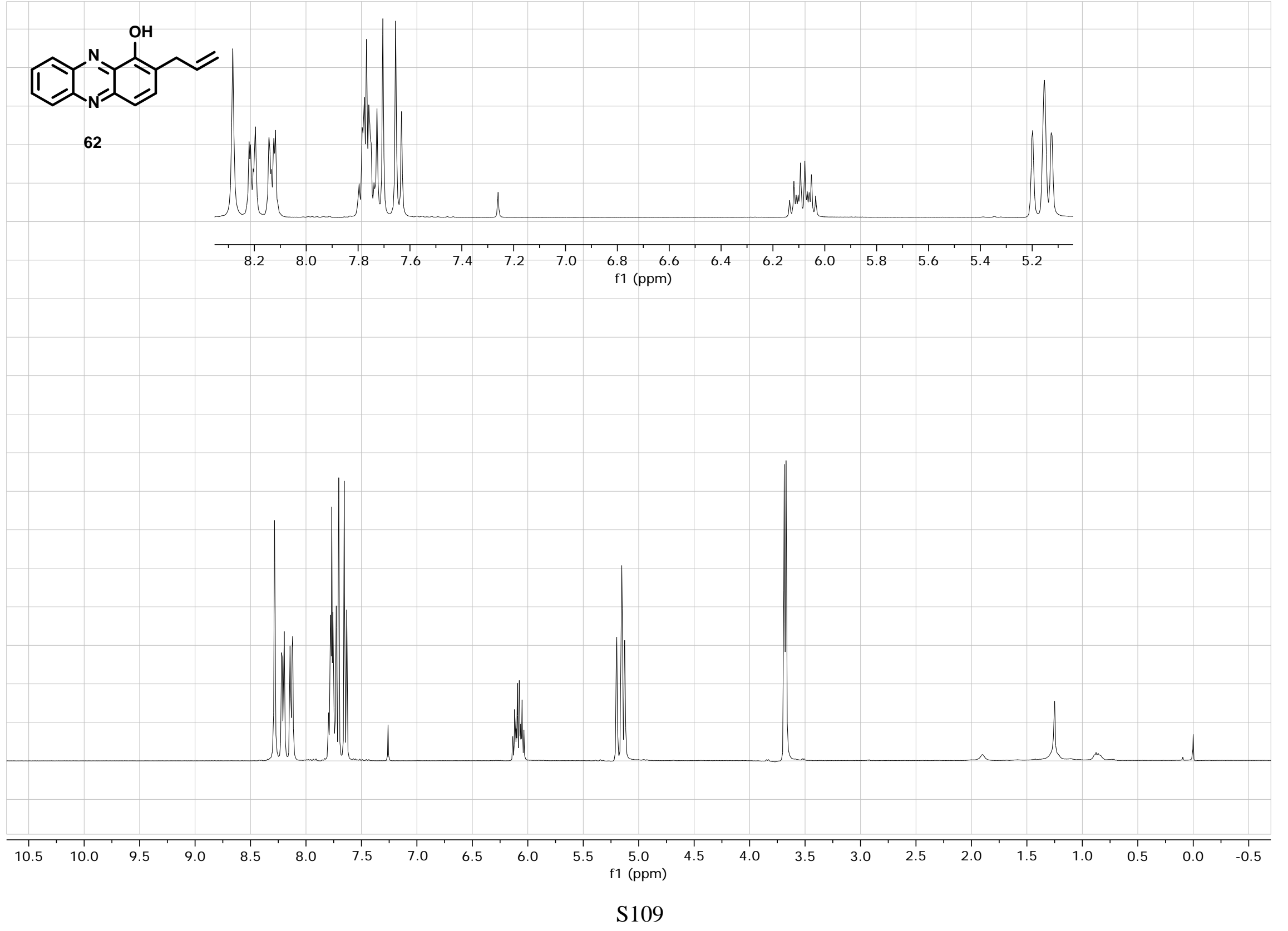




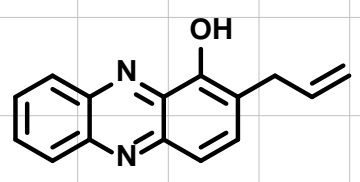

62

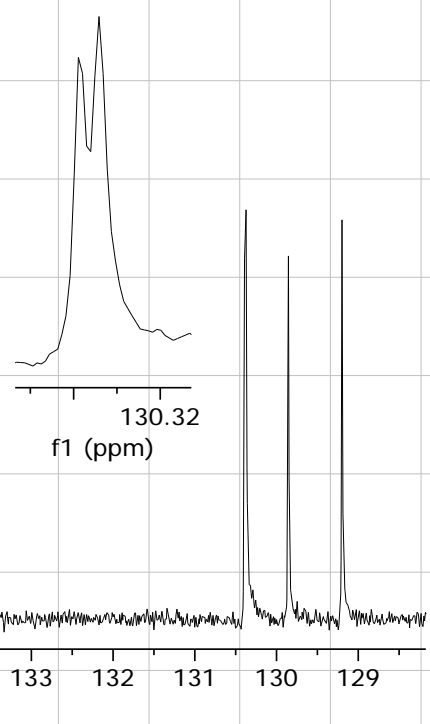

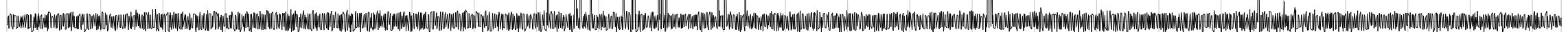



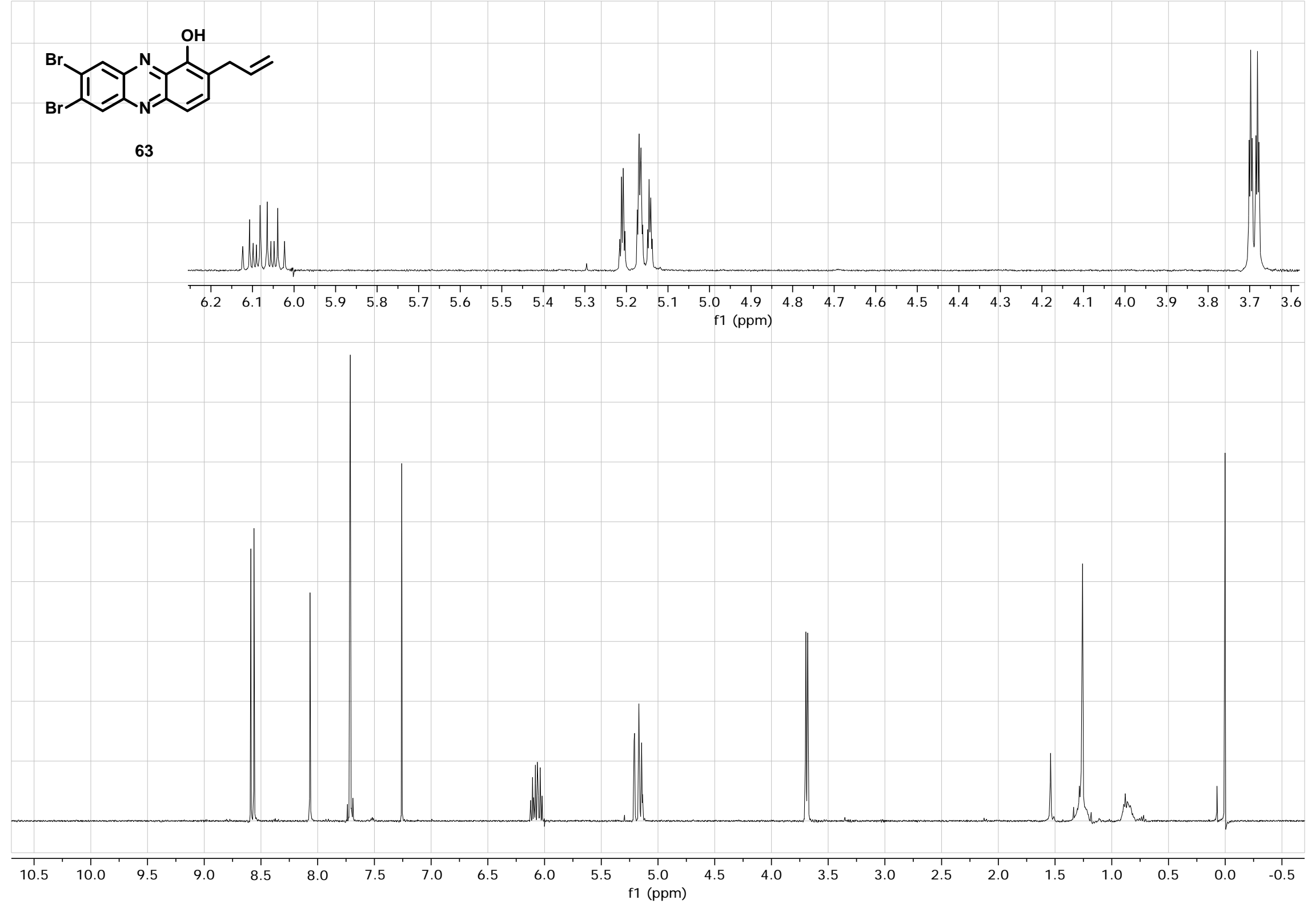


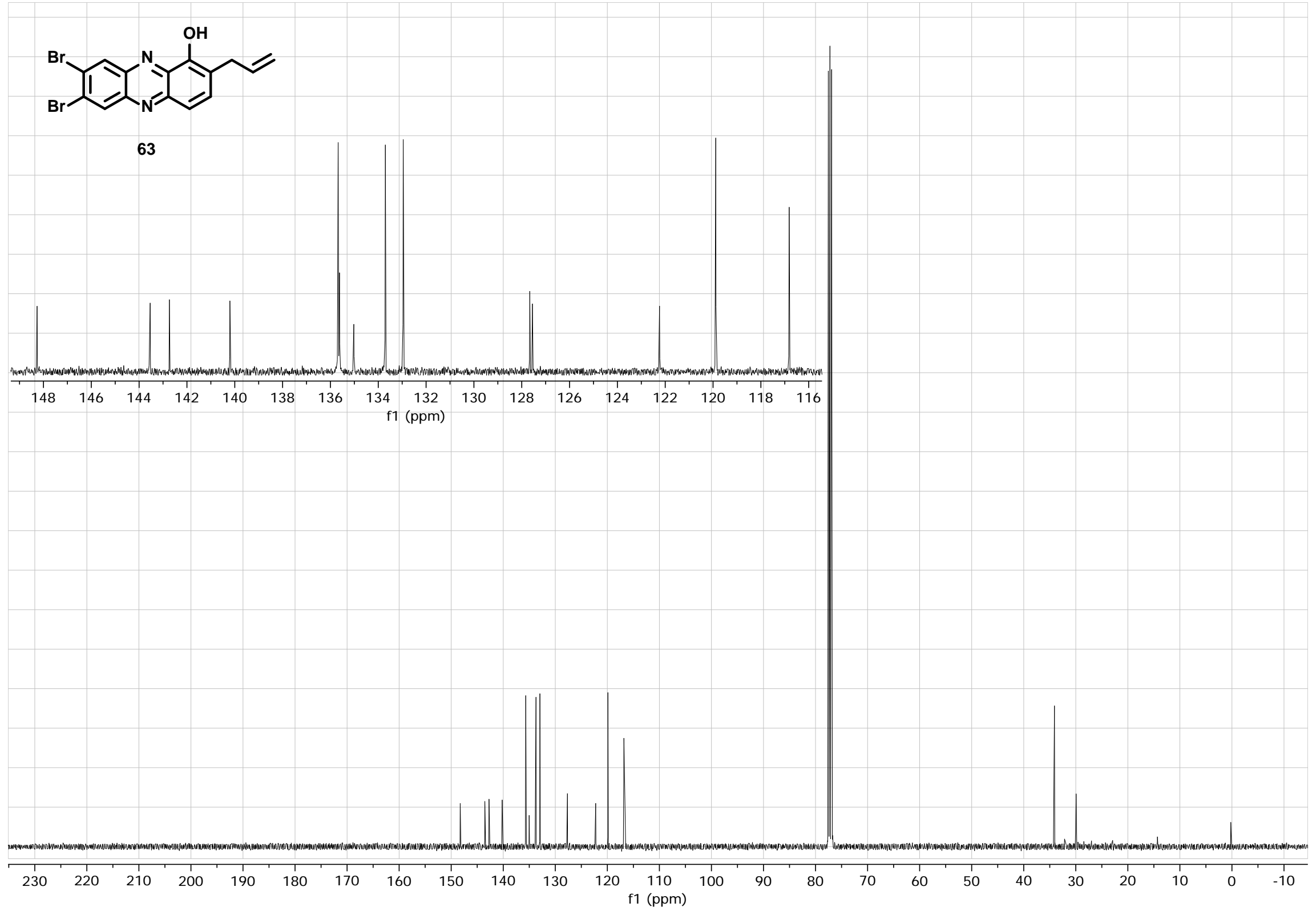

S112 